\title{
Evolutionary demography of structured two-sex populations and sex ratios
}

by

Esther Shyu

B.S., California Institute of Technology, 2010

Submitted in partial fulfillment of the requirements for the degree of

Doctor of Philosophy

at the

MASSACHUSETTS INSTITUTE OF TECHNOLOGY

and the

WOODS HOLE OCEANOGRAPHIC INSTITUTION

September 2015

(C)2015 Esther Shyu.

All rights reserved.

The author hereby grants to MIT and WHOI permission to reproduce and to distribute publicly paper and electronic copies of this thesis document in whole or in part in any medium now known or hereafter created.

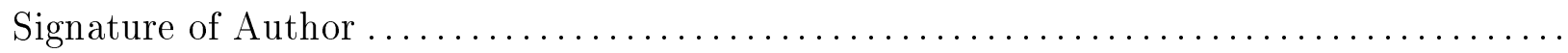
Joint Program in Oceanography and Applied Ocean Science and Engineering Massachusetts Institute of Technology

Woods Hole Oceanographic Institution July 27, 2015

Certified by

Dr. Hal Caswell Thesis Supervisor

Accepted by

Professor Martin F. Polz

Chair, Joint Committee for Biological Oceanography Massachusetts Institute of Technology Woods Hole Oceanographic Institution 


\title{
Evolutionary demography of structured two-sex populations and sex ratios
}

by

\author{
Esther Shyu
}

Submitted to the MIT-WHOI Joint Program in Oceanography and Applied Ocean Science and Engineering on July 27, 2015, in partial fulfillment of the requirements for the degree of Doctor of Philosophy in Biological Oceanography.

\begin{abstract}
Males and females may differ in stage-specific survival, maturation, fertility, or mating availability. These demographic differences, in turn, affect population growth rates, equilibrium structure, and evolutionary trajectories. Models considering only a single sex cannot capture these effects, motivating the use of demographic two-sex models for sexually reproducing populations.

I developed a new two-sex modeling framework that incorporates population structure and multiple life cycle processes through transition rate matrices. These models can be applied to a variety of life histories to address both ecological and evolutionary questions. Here, I apply the model to the effects of sex-biased harvest on populations with various mating systems.

Demographic considerations also affect evolutionary projections. I derived matrix calculus expressions for key evolutionary quantities in my two-sex models, including the invasion fitness, selection gradient, and second derivatives of growth rates (which have many applications, including the classification of evolutionary singular strategies). I used these quantities to analyze the evolution of the primary sex ratio, under various sex- and stage-specific offspring costs and maternal conditions.

Demographic two-sex models lend insight into complex, and sometimes counterintuitive, results that are not captured by models lacking population structure. These findings highlight the importance of demographic structure in ecology and evolution.
\end{abstract}

Thesis Supervisor: Dr. Hal Caswell

Title: Scientist Emeritus, Department of Biology, Woods Hole Oceanographic Institution 


\section{Acknowledgments}

This work was supported by a National Science Foundation Graduate Research Fellowship under Grant 1122374, NSF Grants DEB1145017 and DEB1257545 (to H. Caswell), Advanced Grant 322989

from the European Research Council (to H. Caswell), and the Woods Hole Oceanographic Institution Academic Programs Office. 


\section{Contents}

1 Introduction $\quad 11$

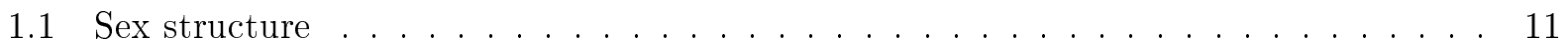

1.2 Two-sex models . . . . . . . . . . . . . . . . . . . . . . 13

1.3 Demography in adaptive dynamics . . . . . . . . . . . . . . . . 13

1.4 Thesis overview . . . . . . . . . . . . . . . . . . . . . . . . . . 14

2 A stage-classified two-sex model incorporating multiple life cycle processes 19

2.1 Abstract . . . . . . . . . . . . . . . . . . . . . . . . 19

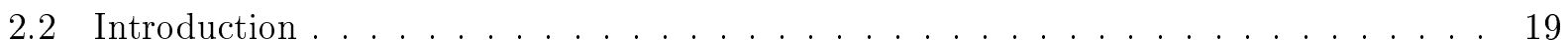

2.3 Model development . . . . . . . . . . . . . . . . . . . . . . 21

2.3.1 Incorporating sex and stage structure . . . . . . . . . . . . . 21

2.3.2 Incorporating life cycle processes . . . . . . . . . . . . . . . . . 22

2.3.3 Modeling the mating process . . . . . . . . . . . . . . . . . 22

2.3.4 Frequency-dependent dynamics . . . . . . . . . . . . . . . . . . . 24

2.4 A 5 -stage BMMR matrix model . . . . . . . . . . . . . . . . . . 25

2.5 Case study: sex-biased harvest in various mating systems . . . . . . . . . . 27

2.5.1 Monogamy $(h=1) \ldots \ldots \ldots \ldots \ldots \ldots \ldots$

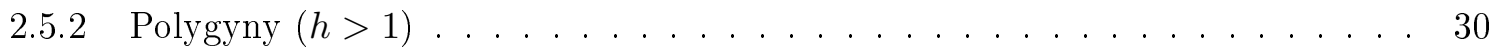

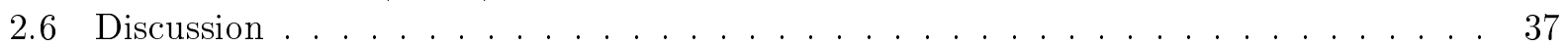

2.6.1 BMMR matrix models in discrete time . . . . . . . . . . . . . . 38

2.7 Acknowledgments . . . . . . . . . . . . . . . . . . . 40

2.A Averaging transition rate matrices . . . . . . . . . . . . . . . 43

2.B Derivatives of a frequency vector . . . . . . . . . . . . . . . 44

2.C BMMR matrices for a polygynous system . . . . . . . . . . . . . . . 44

3 A demographic model for sex ratio evolution and the effects of sex-biased $\begin{array}{ll}\text { offspring costs } & 47\end{array}$

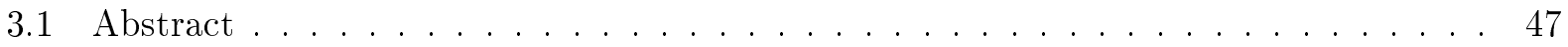

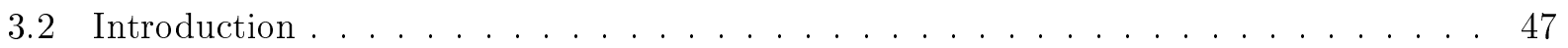

3.3 Model and methods . . . . . . . . . . . . . . . . . . . . 50

3.3.1 The two-sex matrix model . . . . . . . . . . . . . . . . 50

3.3.2 Evolutionary analysis with adaptive dynamics . . . . . . . . . . . . . 53

3.3.3 Second derivatives of invasion fitness . . . . . . . . . . . . . . 56

3.4 Case studies: sex-biased offspring costs . . . . . . . . . . . . . . . . . . 59

3.4.1 Case 0: identically costly sexes . . . . . . . . . . . . . . 63

3.4 .2 Case 1: offspring resource costs . . . . . . . . . . . . . 63

3.4.3 Case 2: offspring mortality during parental investment . . . . . . . . . . 65 
3.4.4 Case 3: offspring mortality after parental investment . . . . . . . . . . 68

3.4.5 Case 4: parental mortality . . . . . . . . . . . . . . . 70

3.4.6 Evolutionary and convergence stability of the SS sex ratio . . . . . . . . . 72

3.4 .7 Primary reproductive value ratios . . . . . . . . . . . . . 73

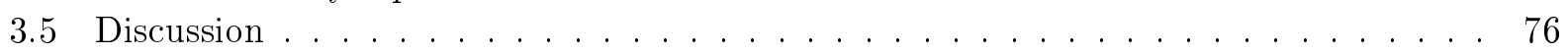

3.5.1 The importance of union formation . . . . . . . . . . . . . 76

3.5.2 The role of reproductive value . . . . . . . . . . . . . 78

3.5.3 Extensions and caveats . . . . . . . . . . . . . . . . . 79

3.6 Acknowledgments . . . . . . . . . . . . . . . . . . . 81

3.A Relationship between second derivatives at singular strategies . . . . . . . . 85

3.B Calculating mixed second derivatives . . . . . . . . . . . . . 85

4 Calculating second derivatives of population growth rates for ecology and $\begin{array}{lr}\text { evolution } & \mathbf{8 9}\end{array}$

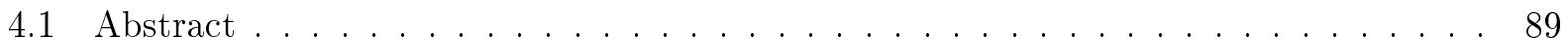

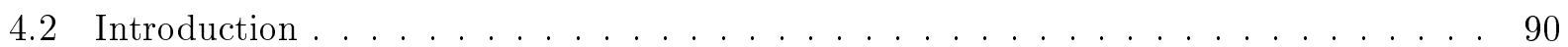

4.2.1 Applications of second derivatives of growth rates . . . . . . . . . . . . 91

4.2.2 Calculating second derivatives of growth rates . . . . . . . . . . . . . . 93

4.2 .3 Notation . . . . . . . . . . . . . . . . . . . . . 93

4.3 Matrix calculus . . . . . . . . . . . . . . . . . . . . 94

4.3.1 Matrix calculus notation . . . . . . . . . . . . . . 94

4.3 .2 The identification theorems . . . . . . . . . . . . . . 95

4.4 Second derivatives of growth rates . . . . . . . . . . . . . . . . 95

4.4.1 Second derivatives of the discrete-time growth rate $\lambda \ldots \ldots$. . . . . . . 96

4.4.2 Second derivatives of the invasion exponent $r: H[r ; \operatorname{vec} \mathbf{A}]$ and $H[r ; \boldsymbol{\theta}] \ldots 99$

4.4.3 Second derivatives of the net reproductive rate $R_{0} \ldots \ldots \ldots$

4.5 Case study: Calathea ovandensis . . . . . . . . . . . . . . . . . 102

4.5.1 Study species . . . . . . . . . . . . . . . . . . . . . . . . . . . . . . . . . . . . . . . .

4.5.2 Second derivatives of $\lambda \ldots \ldots \ldots \ldots \ldots \ldots$

4.5.3 Applications of the second derivatives . . . . . . . . . . . . 104

4.6 Discussion . . . . . . . . . . . . . . . . . . . . 106

4.7 Acknowledgments . . . . . . . . . . . . . . . . . . . 108

4.A First differentials of $\lambda, \mathbf{w}$, and $\mathbf{v} \ldots \ldots \ldots \ldots \ldots \ldots$

4.A.1 First differential of the dominant eigenvalue: $d \lambda \ldots \ldots \ldots \ldots$

4.A.2 First differential of the right eigenvector: $d \mathbf{w} \ldots \ldots \ldots \ldots \ldots$

4.A.3 First differential of the left eigenvector: $d \mathbf{v} \ldots \ldots \ldots \ldots \ldots$. . . . . . . . . . .

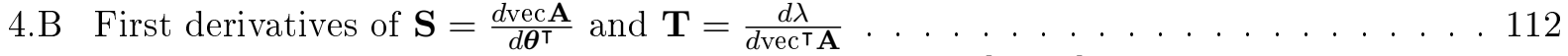

4.C Second derivatives of $R_{0}$ to lower-level parameters: $H\left[R_{0} ; \boldsymbol{\theta}\right] \ldots \ldots \ldots$

4.C.1 Life cycles with only one type of offspring . . . . . . . . . . . . . 115

4.D Sensitivity of the stochastic growth rate . . . . . . . . . . . 116

4.E Commutation matrix code . . . . . . . . . . . . . . . . 117

5 The evolution of facultative sex ratios with two maternal conditions 119

5.1 Abstract . . . . . . . . . . . . . . . . . . . . . 119

5.2 Introduction . . . . . . . . . . . . . . . . . . . . 119

5.3 Model and methods . . . . . . . . . . . . . . . . . . 122

5.3.1 A two-sex matrix model with multiple maternal conditions . . . . . . . . . . 122

5.3 .2 Mating preferences . . . . . . . . . . . . . . . 124 
5.3.3 Multidimensional adaptive dynamics . . . . . . . . . . . . . . 126

5.4 Case studies . . . . . . . . . . . . . . . . . . . . . . . . . 130

5.5 Case 1: Maternal age . . . . . . . . . . . . . . . . . 131

5.5 .1 Model . . . . . . . . . . . . . . . . . . . . . . 132

5.5.2 Results (Case 1a: Parental mortality cost) . . . . . . . . . . . . . . . 135

5.5.3 Results (Case 1b: Parental resource cost) . . . . . . . . . . . . . . 138

5.6 Case 2: Maternal quality . . . . . . . . . . . . . . . . . 138

5.6 .1 Model . . . . . . . . . . . . . . . . . . . . . 141

5.6 .2 Calculating variance in reproductive success . . . . . . . . . . . . . . 144

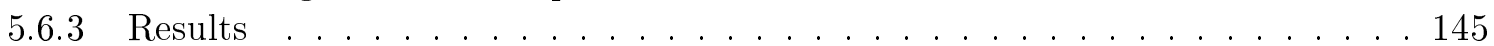

5.7 Discussion . . . . . . . . . . . . . . . . . . . . . 150

5.8 Acknowledgments . . . . . . . . . . . . . . . . . . . . . 152

5.A Reproductive value ratios at singular strategies . . . . . . . . . . 156

5. A.1 Interior SS (Type 1) . . . . . . . . . . . . . . . . . 157

5$. A.2 Boundary SS (Type $2-5) \ldots \ldots \ldots \ldots \ldots \ldots \ldots$

5.B Stability of $2 \mathrm{D}$ singular strategies . . . . . . . . . . . . . . . 159

5. B.1 Evolutionary stability . . . . . . . . . . . . . . . . 159

5. B.2 Convergence stability . . . . . . . . . . . . . . . . 160

6 Concluding Remarks 163

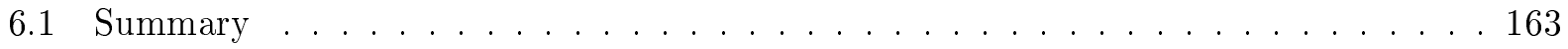

6.2 Extensions . . . . . . . . . . . . . . . . . . . . . . . 164

6.2 .1 Alternative mating strategies . . . . . . . . . . . . . . 164

6.2 .2 Evolution under harvest . . . . . . . . . . . . . . . . 165 


\section{Chapter 1}

\section{Introduction}

Demography, as the study of life cycle and population structure, explores the interaction between individuals and populations (Caswell 2001). Individuals may vary in sex, age, size, life cycle phase, condition, and many other characteristics. These individual differences are demographic or i-state variables (Metz and Diekmann 1986) that denote various demographics or i-states — stages or segments of the population with shared characteristics. The distribution of i-state variables is described by p-state variables, such as age and stage distributions, that ultimately affect properties like population growth rates or equilibria.

Through their effects on high-level population properties, demographic factors play important parts in ecological processes, as well as evolutionary ones. Demography and evolution are connected through many links of mutual feedback. Population dynamics drive genotype propagation, and selective pressures change as evolving populations reshape their environments. Fitness depends on how stage-specific survival and reproduction influence offspring production, and the evolution of traits with demographic consequences (e.g., life history strategies) affects population dynamics that feed back into evolution. As a result, there have been many calls to improve the integration of demographic and evolutionary factors in biological research (e.g., Metcalf and Pavard 2007, Kokko 2007).

\section{$1.1 \quad$ Sex structure}

In sexually reproducing populations, a demographic factor of particular interest is sex structure, typically in terms of males and females. The sex ratio (relative numbers of each sex) affects mating opportunities and subsequent reproduction, ultimately determining the population's growth rate.

As discussed below, sex structure is central to a variety of management and conservation issues. In each case, demographic factors such as sex, size, and condition are important considerations for making population and evolutionary projections. 
- Sport and trophy hunting. Harvest by hunters is often male-biased and significantly exceeds natural mortality levels (e.g., Festa-Bianchet 2003, Milner 2007). Such harvest may alter population structure, reproductive strategies, and trait evolution. Other species respond to sex-biased harvest in even more extreme ways. African lions, for instance, commit infanticide when male harem leaders are killed (Whitman et al. 2014), which exacerbates the effects of male harvest on population growth.

Sex-biased hunting strategies are often also stage-biased, as larger (older) males with well-developed adult characteristics (e.g., antlers, horns, or tusks) are the most desirable targets. Additionally, changes in fecundity with age may drastically alter the outcome of sex-biased harvest strategies (e.g., Ginsberg 1994 for African impala). All these factors motivate the use of a demographic two-sex model for projecting harvest effects.

- Conservation of threatened and endangered species. Population growth may be restricted by the availability of a given sex. In polygynous mating systems (one male mates with many females), for example, total reproduction is limited by the number of breeding females. Artificially manipulating the sex ratio in favor of the more limiting sex could thus increase the viability of small, declining, or otherwise vulnerable populations (Wedekind 2002).

However, parental or environmental conditions may affect the proportion of male and female offspring produced (Trivers and Willard 1973). Endangered kakapo parrots, for instance, produce more sons when well-fed, so providing wild birds with supplementary food induces overly male-biased sex ratios unfavorable for breeding (Clout et al. 2002). While failing to anticipate condition-dependent sex ratio shifts can stymie conservation attempts, factors like multiple parental conditions may be challenging to incorporate into evolutionary projections without an explicitly-demographic model.

- Biocontrol and pest management. Just as sex ratios can be manipulated to promote endangered species, they can be manipulated to control unwanted pests. Sterile insect technique (SIT, reviewed in Robinson 1983, Dyck et al. 2005) is a biocontrol method that reduces pest insect reproduction by altering natural sex ratios - specifically, the ratio of females to fertile males. Male insects are raised and rendered sterile in laboratory environments, then released to compete with wild fertile males for mates. This method has successfully controlled, and even eradicated, parasites and agricultural pests such as screwworms and fruit flies in North America (Dyck et al. 2005).

The efficacy of a SIT program depends on the population's sex structure, male stage structure (i.e., the relative abundances of sterile and fertile males), and the insect mating process. The competition between sterile and wild males could be captured by a demographic two-sex model with different female mating preferences for different types of males. Additionally, SIT release programs are often used in tandem with other stage-specific management strategies, such as 
larvicides or adulticides (Dyck et al. 2005), and would accordingly benefit from demographic models with life cycle structure.

\subsection{Two-sex models}

Models that neglect sex structure only consider a single, dominant sex (typically females). As a result, they implicitly assume that both sexes have identical vital rates and that the availability of the neglected sex (i.e. males) does not affect fertility.

In reality, both of these assumptions are frequently violated. Due to sexual dimorphism, males and females may differ in vital rates, behavior, and even harvest pressure (e.g., Caswell 2001, Rankin and Kokkko 2007). Additionally, population, environmental, and evolutionary changes can vary the limiting sex over time (e.g., Hardy 2002, Miller and Inouye 2011). Under such circumstances, one-sex models may be insufficient to model population dynamics, making two-sex models a desirable alternative.

Because age- and stage-structure may have significant effects on two-sex population dynamics (e.g., Sundelöf and Aberg 2006, Ginsberg 1994), an explicitly demographic two-sex model that incorporates both multiple sexes and stages would be especially valuable. Additionally, multiple demographic factors, including sex-biased mortality, have been speculated to affect the evolution of the primary sex ratio (proportion of offspring that are born male) (Fisher 1930). Because reproduction is shaped by the entire life cycle, sex ratio theory would benefit from two-sex models with life cycle structure. However, few, if any, studies have examined sex ratio evolution with an explicitly demographic two-sex model.

Ultimately, such models are needed to capture the effects of sex (and other population structure) on ecology and evolution. As a result, my overarching objective is to develop a general demographic modeling framework that can be used to explore two-sex population dynamics and the evolution of traits like sex ratio.

\subsection{Demography in adaptive dynamics}

Evolutionary analyses often focus on finding evolutionarily stable strategies (ESSs) as potential evolutionary endpoints (e.g., Charnov 1982, Hardy 2002, Otto and Day 2007). However, additional methods are needed to determine whether the population will ultimately converge to an ESS, or if other evolutionary outcomes occur in the long run. To this end, adaptive dynamics (AD) identifies and characterizes evolutionarily singular strategies in terms of both evolutionary and convergence stability (Geritz et al. 1998).

While AD is useful for modeling frequency-dependent evolution, of which sex ratio evolution is a prominent example, it often assumes that individuals are asexual and phenotypically identical, save for the evolving trait of interest (Waxman and Gavrilets 2005). Previous extensions of AD 
to structured population models have utilized a variety of approaches. ESS analysis, in particular, has been applied to populations with multiple developmental stages (e.g., models including both juveniles and adults in Van Dooren and Metz 1998, Hardy 2002, and Otto and Day 2007), sizes (e.g., via integral projection models in Childs et al. 2003, Metcalf et al. 2008), and spatial locations (e.g., with multiple habitats in Otto and Day 2007 and dispersing metapopulations in Gyllenberg and Metz 2001). More general AD approaches, including singular strategy characterization and pairwise invasion plots, have also used structured population models to some extent (e.g., Claessen and Dieckmann 2002, Metcalf et al. 2008 for size-structured populations, Parvinen 2002, 2006 for metapopulations).

This thesis develops evolutionary analyses for a broad range of two-sex, stage-structured populations. I have integrated adaptive dynamics methods into my demographic two-sex modeling framework, and have used these methods to explore the evolution of sex ratio under various conditions.

\subsection{Thesis overview}

My thesis explores how demographic considerations in two-sex models affect both population properties, such as growth and equilibrium structure, and the evolution of traits, namely the primary sex ratio.

The four chapters are as follows:

- Chapter 2 introduces the underlying demographic two-sex population model.

- Chapter 3 uses the two-sex model to explore sex ratio evolution when male and female offspring differ in their costs.

- Chapter 4 derives second derivatives for population growth rates, which are used to characterize the stability of evolutionary outcomes in adaptive dynamics.

- Chapter 5 considers the evolution of facultative sex ratios that depend on maternal condition.

\section{Chapter 2 - A stage-classified two-sex model incorporating multiple life cycle processes.}

Chapter 2 introduces a new two-sex modeling framework that incorporates multiple sexes, stages, and life cycle processes.

In this model, population dynamics are given by a series of rate matrices that describe mating, reproduction, and transitions between stages. Matrix models like these classify individuals into stages based on sex, age, or any other i-state variables of interest, and link demographic life cycle information (i.e. stage-specific vital rates in the projection matrix) to population-level properties important for evolution (e.g., long-term growth rates or equilibria) (Caswell 2001).

The resulting model can be used to investigate the effects of stage and sex structure, including sex-biased vital rates such as differential mortality or maturation. As an example, I project the 
long-term dynamics of populations with various mating systems and their responses to sex-biased harvest. I find that mating factors, including both harem size and persistence, affect not only unharvested population growth, but also the responses of growth rate and sex ratio to sex-biased harvest.

Chapter 3 - A demographic model for sex ratio evolution and the effects of sex-biased offspring costs. Chapter 3 presents a demographic approach to sex ratio evolution that combines adaptive dynamics with the two-sex matrix model presented in Chapter 2.

The methods described in this chapter allow the incorporation of any population structure, including multiple sexes and stages, into evolutionary projections. In particular, I have derived key adaptive dynamics quantities (such as the invasion fitness, selection gradient, and second derivatives for singular strategy characterization) for the two-sex matrix model.

I also compare how four different interpretations of sex-biased offspring costs affect sex ratio evolution. The analyses calculate singular strategies that depend on sex and stage differences, and determine their evolutionary and convergence stability using methods derived in Chapter 4. I find that demographic differences affect evolutionary outcomes, and that, in some cases, the sex ratios that result contradict the widely-held conclusions of previous models that neglect demographic structure.

Chapter 4 - Calculating second derivatives of population growth rates for ecology and evolution. Chapter 4 derives a suite of matrix calculus formulae for the second derivatives of population growth rates. This chapter previously appeared in Methods and Ecology and Evolution (2014, Creative Commons Attribution license).

I focus on three population growth rate measures: the discrete-time growth rate $\lambda$, the continuous-time growth rate $r=\log \lambda$, and the net reproductive rate $R_{0}$, which measures per-generation growth. I present their derivatives with respect to both projection matrix entries and to lower-level parameters affecting those matrix entries.

These second derivatives have a variety of ecological and evolutionary applications, including quantifying the response of sensitivity results to perturbations, classifying types of selection, and calculating sensitivities of the stochastic growth rate. I illustrate several applications with a case study for the tropical herb Calathea ovandensis. These second derivatives also provide methods to characterize the stability of evolutionarily singular sex ratio strategies in Chapters 3 and 5 .

\section{Chapter 5 - The evolution of facultative sex ratios with two maternal conditions.}

Chapter 5 extends the model and calculations described in Chapter 3 to the evolution of sex ratios that depend on maternal condition.

I specifically consider cases where individuals differ in age or quality, and present several two-sex demographic models that include two maternal conditions with independent sex ratios. The overall sex ratio strategy is a vector trait with two simultaneously evolving components. Using 
multidimensional adaptive dynamics, I analyze both the transient and long-term evolution of this bivariate sex ratio.

I find that the bivariate sex ratio has a wide range of evolutionary behavior, including cases where it converges to a line of selectively-neutral strategies. The sex ratio may also evolve so that one maternal condition produces exclusively male or female offspring. In particular, the sexes preferred by mothers of different qualities depend on the relative reproductive value ratios for each sex.

\section{Bibliography}

[1] Caswell H. 2001. Matrix population models. Sunderland: Sinauer Associates, Inc.

[2] Charnov EL. 1982. The theory of sex allocation. Princeton: Princeton University Press.

[3] Childs DZ, Rees M, Rose KE, Grub PJ, Ellner SP. 2003. Evolution of complex flowering strategies: an age- and size-structured integral projection model. Proceedings of the Royal Society B. 270: 1829-1838.

[4] Claessen D, Dieckmann U. 2002. Ontogenetic niche shifts and evolutionary branching in size-structured populations. Evolutionary Ecology Research. 4: 189-217.

[5] Clout, MM, Elliot GP, Robertson BC. 2002. Effect of supplementary feeding on the offspring sex ratio of kakapo: a dilemma for the conservation of a polygynous parrot. Biological Conservation. 107, 13-18.

[6] Dyck VA, Hendricks J, Robinson AS, eds. 2005. Sterile insect technique: Principles and practice in area-wide integrated pest management. Dordrecht, Netherlands: Springer.

[7] Festa-Bianchet M. 2003. Exploitative wildlife management as a selective pressure for life-history evolution of large mammals. In Animal Behavior and Wildlife Conservation (eds Festa-Bianchet M, Apollonio M). Washington, D.C.: Island Press. pp. 191-210.

[8] Fisher RA. 1930. The Genetical Theory of Natural Selection. Oxford: Oxford University Press.

[9] Geritz SAH, Kidsi É, Meszéna G, Metz JAJ. 1998. Evolutionarily singular strategies and the adaptive growth and branching of the evolutionary tree. Evolutionary Ecology. 12: 35-57.

[10] Ginsberg JS, Milner-Gulland EJ. 1994. Sex-based harvesting and population dynamics in ungulates: implications for conservation and sustainable use. Conservation Biology. 18: $157-166$.

[11] Gyllenberg M, Metz JAJ. 2001. On fitness in structured metapopulations. Journal of Mathematical Biology. 43: 545-560.

[12] Hardy ICW, editor. 2002. Sex ratios: concepts and research methods. Cambridge: Cambridge University Press.

[13] Kokko H, López-Sepulcre A. 2007. The ecogenetic link between demography and evolution: can we bridge the gap between theory and data? Ecology Letters. 10:773-782. 
[14] Metcalf CJE, Pavard S. 2007. Why evolutionary biologists should be demographers. Trends in Ecology and Evolution. 22: 205-212.

[15] Metcalf CJE, Rose KE, Childs DZ, Sheppard AW, Grubbs PJ, Rees M. 2008. Evolution of flowering decisions in a stochastic, density-dependent environment. PNAS. 105: 10466-10470.

[16] Metz JAJ, Diekmann O. 1986. The dynamics of physiologically structured populations. Berlin: Springer-Verlag.

[17] Miller TEX, Inouye BD. 2011. Confronting two-sex demographic models with data. Ecology. 92: 2141-2151.

[18] Milner JM, Nilsen EB, Andreaseen HP. 2007. Demographic side effects of selective hunting in ungulates and carnivores. Conservation Biology. 21: 36-47.

[19] Otto SP, Day T. 2007. A biologist's guide to mathematical modeling in ecology and evolution. Princeton: Princeton University Press.

[20] Parvinen K. 2002. Evolutionary branching of dispersal strategies in structured metapopulations. Journal of Mathematical Biology. 45: 106-124.

[21] Parvinen K. 2006. Evolution of dispersal in a structured metapopulation model in discrete time. Bulletin of Mathematical Biology. 68: 655-678.

[22] Rankin DJ, Kokko H. 2007. Do males matter? The role of males in population dynamics. Oikos. 116: 335-348.

[23] Robinson AS. 1983. Sex-ratio manipulation in relation to insect pest control. Annual Review of Genetics. 17: 191-214.

[24] Sundelöf A, Åberg P. 2006. Birth functions in stage structured two-sex models. Ecological Modelling. 193: 787-795.

[25] Trivers RL, Willard DE. 1973. Natural selection of parental ability to vary the sex ratio of offspring. Science. 179: 90-92.

[26] Van Dooren TJM, Metz JAJ. 1998. Delayed maturation in temporally structured populations with non-equilibrium dynamics. Journal of Evolutionary Biology. 11: 41-62.

[27] Waxman D, Gavrilets S. 2005. 20 questions on adaptive dynamics. Journal of Evolutionary Biology. 18: 1139-1154.

[28] Wedekind C. 2002. Manipulation sex ratios for conservation: short-term risks and long-term benefits. Animal Conservation. 5: 13-20.

[29] Whitman K, Starfield AM, Quadling HS, Packer C. 2004. Sustainable trophy hunting of African lions. Nature. 428: 175-177. 


\section{Chapter 2}

\section{A stage-classified two-sex model incorporating multiple life cycle}

\section{processes}

\subsection{Abstract}

Models of sexually-reproducing populations that consider only a single sex cannot capture the effects of sex-specific differences and mate availability. Explicitly demographic two-sex models enable a more rigorous analysis of population dynamics. To this end, we present a new framework for modeling two-sex populations that incorporates multiple sexes, stages, and life cycle events through a series of transition rate matrices. We illustrate this framework with a case study for the effects of sex-biased harvest on populations with various mating systems. Our results emphasize that two-sex demographic factors play a major part in a population's ecological dynamics and harvest response.

Keywords: two-sex models, demography, matrix population models, birth matrix-mating rule, BMMR, sex-biased harvest

\subsection{Introduction}

Models of sexually-reproducing populations that consider a only single sex (typically females) implicitly assume that both sexes have identical vital rates and that the availability of the neglected sex (typically males) does not affect fertility (Pollard 1974, Caswell 2001, Ianelli et. al 2005).

In reality, both these assumptions are frequently violated. Males and females often differ significantly in terms of fertilities and mortalities (Kuczynski 1932, Pollak 1990, Jenouvrier et al. 2010), developmental schedules (Caswell 2001), behavioral interactions (Rankin and Kokko 2007), dispersal patterns (Miller et al. 2011), and selective harvest pressures (Ginsberg 1994). Additionally, ecological, environmental, and evolutionary changes may vary the most limiting sex 
over time (Hardy 2002, Miller and Inouye 2011). One-sex models are insufficient for modeling populations in all these cases.

In response to growing concerns over discrepancies in male and female reproductive rates (Karmel 1947), early dynamical models with sex structure were introduced in the late 1940s. Pollard (1948) used coupled Lotka integral equations, considering female births to males and male births to females in order to reconcile the growth rates of both sexes. Kendall (1949) introduced a system of ordinary differential equations for males and females (and later, married couples), and was the first to incorporate nonlinear interactions between the sexes via a mating term. Subsequent models considered other nonlinear mating functions (Pollard 1973, Yellin and Samuelson 1974) and couple dissolution through death and divorce (Hadeler et. al 1988).

Extensions to age-structured populations were made by Fredrickson (1971) and Hoppensteadt (1975), who allowed birth and death rates, as well as couple formation and divorce rates, to depend on age and sex. Haedler later included the "age" (duration) of married pairs (1988) and maturation delays (1993). Age-structured mating functions have similarly been proposed (Martcheva and Milner 2001). Many of these models use continuous-time equations that incorporate age structure through coupled McKendrick-von Foerster partial differential equations (Keyfitz 1972, Fredrickson 1971, Hoppensteadt 1975, Hadeler 1989, Hadeler 1993), though discrete-time two sex matrix models have also been developed (Caswell and Weeks 1986).

Another approach to formulating two-sex models with age structure was proposed by Pollak (1986, 1987, 1990). Pollak's birth matrix-mating rule (BMMR) focuses on the mating, births, and life cycle transition processes that repeat every generation, and has three main components:

1. A mating rule function that projects the number of matings $u_{i j}$ between males of age $i$ and females of age $j$.

2. A birth matrix whose entries $b_{i j}$ are the expected number of male and female offspring produced by each age $i$ male and age $j$ female mating.

3. Sex-specific mortality schedules, which could be generalized to include other age-specific life cycle transitions.

BMMR is a useful approach for describing two-sex populations because it can specify age (and, more generally, stage) structure over all parts of the life cycle. This structure, in turn, can have significant effects on two-sex population dynamics (e.g., Sundelöf and Aberg 2006, where the addition of size-specific birth functions affects growth rate and reproductive output) and recommended management strategies (e.g., Ginsberg 1994, where incorporating age-specific fecundity changes the outcomes of sex-biased harvest).

Using BMMR as a conceptual basis, we present a more general demographic two-sex modeling framework with several extensions. Though Pollak primarily considered age-sex structure, we also incorporate detailed life cycle structure, most notably multiple stages and various types of mated 
pairs or harems. The original BMMR model was also formulated as a series of mappings between population states in different time periods. Here, we use a series of comprehensive transition rate matrices to more fully describe various life cycle processes. Given a variety of biologically feasible functions for the mating rule, our two-sex matrix models converge to a stable stage distribution with an exponential growth rate. As we demonstrate with a case study for sex-biased harvest, these long-term population properties depend on the system's underlying demographic structure.

\subsection{Model development}

We use two-sex, continuous-time matrix models to incorporate stage structure and life cycle processes. The mating, births, and other transition processes from the BMMR framework are described by separate rate matrices. The mating process, in particular, is modeled by generalized weighted mean mating functions that satisfy biologically relevant criteria. The resulting BMMR matrix models are nonlinear and have frequency-dependent dynamics that converge to an exponential growth rate and stable stage distribution (constant distribution of stage frequencies).

\subsubsection{Incorporating sex and stage structure}

We incorporate sex and stage structure by using a matrix population model, which classifies individuals into separate stages based on age, developmental state, sex, reproductive status, or any other variables of interest. Stage densities are projected forward in time by a projection matrix that contains the demographic rates or probabilities characterizing survival, reproduction, and transitions between stages (Caswell 2001). The properties of this projection matrix provide information about the population as a whole, thereby linking demographic life cycle information (i.e., the stage-specific vital rates in the matrix entries) to population-level properties important for ecology and evolution (e.g., growth rates or stage distributions).

A population with $s$ stages is described by a $s \times 1$ population vector $\mathbf{n}(t)$, the entries of which are the densities of each stage at time $t$. In a two-sex population, $\mathbf{n}(t)$ would contain male stages, female stages, and mated stages (unions) that could include married couples or breeding harems.

The population vector for a two-sex population with mating adults and nonmating juveniles, for instance, could have the form:

$$
\mathbf{n}(t)=\left(\begin{array}{c}
\text { juvenile males } \\
\text { adult males } \\
\text { juvenile females } \\
\text { adult females } \\
\text { adult unions }
\end{array}\right)
$$

Additional ages, sizes, union types, or other stages of interest can be added as additional entries in the population vector. 
The product of the population vector and a $s \times s$ projection matrix $\mathbf{A}$ is a system of coupled differential equations:

$$
\frac{d \mathbf{n}}{d t}=\mathbf{A n}(t)
$$

Because this is a continuous-time model, the entries in $\mathbf{A}$ are either transition rates or rates of offspring production.

\subsubsection{Incorporating life cycle processes}

Various life cycle processes are incorporated using a series of transition rate matrices. As in the

BMMR framework, we will specifically consider mating, birth, and transition processes. Each process is described by a separate matrix for the sake of clarity:

1. The mating (union formation) process, where adult males and females organize into reproductive unions, is described by the matrix $\mathbf{U}$.

2. The birth process, where unions produce new offspring, is described by the matrix $\mathbf{B}$.

3. The transition process, which includes other life cycle events like mortality, maturation, or divorce, is described by the matrix $\mathbf{T}$.

Other life cycle processes can be included with additional matrices.

In continuous-time, all these processes occur simultaneously. It can be shown (Appendix 2.A) that the projection matrix $\mathbf{A}$ in (2.2) is the average of the transition rate matrices, e.g.:

$$
\begin{aligned}
\frac{d \mathbf{n}}{d t} & =\frac{1}{3}(\mathbf{T}+\mathbf{B}+\mathbf{U}) \mathbf{n}(t) \\
& =\mathbf{A n}(t)
\end{aligned}
$$

\subsubsection{Modeling the mating process}

The mating process, as described by the union formation matrix $\mathbf{U}$, can be especially challenging to model due to its nonlinearity. Mating depends on the relative numbers of males and females in the population, not all of which may be mature enough or available for breeding (Pollard 1974, Ianelli et al. 2005). As a result, $\mathbf{U}$ depends on the population's sex and stage composition, making $\mathbf{A}$ a function of the population vector $\mathbf{n}(t)$.

The total mating function $M(\mathbf{n})$ gives the rate of union formation (total number of unions formed per unit time), similar to the mating rule in the BMMR framework. Here, "unions" refer to any mated, reproducing units in the population, including both one-to-one male-female pairs and harems with multiple individuals of the same sex. 


$$
M(\boldsymbol{n})=\left[\beta f^{\alpha}+(1-\beta) m^{\alpha}\right]^{1 / \alpha} \text { where } \boldsymbol{n}=[m f]^{\top}
$$

\begin{tabular}{|ccc|cccc|}
\hline Function & $\boldsymbol{U}_{T}(\boldsymbol{n})$ & $(\alpha, \beta)$ & $\mathbf{1}$ & $\mathbf{2}$ & $\mathbf{3}$ & $\mathbf{4}$ \\
$\begin{array}{c}\text { female } \\
\text { dominance }\end{array}$ & $f$ & $(-, 1)$ & $\checkmark$ & $\checkmark$ & & $\checkmark$ \\
\hline $\begin{array}{c}\text { male } \\
\text { dominance }\end{array}$ & $m$ & $(-, 0)$ & $\checkmark$ & $\checkmark$ & & $\checkmark$ \\
\hline minimum & $\min (m, f)$ & $(-\infty,-)$ & $\checkmark$ & $\checkmark$ & $\checkmark$ & $\checkmark$ \\
\hline $\begin{array}{c}\text { geometric } \\
\text { mean }\end{array}$ & $\sqrt{m f}$ & $(0,1 / 2)$ & $\checkmark$ & $\checkmark$ & $\checkmark$ & $\checkmark$ \\
\hline $\begin{array}{c}\text { harmonic } \\
\text { mean }\end{array}$ & $\frac{2 m f}{m+f}$ & $(-1,1 / 2)$ & $\checkmark$ & $\checkmark$ & $\checkmark$ & $\checkmark$ \\
\hline
\end{tabular}

Mating Function Criteria

1. $M$ is nonnegative for all nonnegative $\mathbf{n}$

2. $M$ nondecreasing in $\mathbf{n}$

3. $M=0$ if $m=0$ or $f=0$

4. $M$ is homogeneous of degree 1 in $\mathbf{n}$

Figure 2-1: Mating functions from the generalized weighted mean family (2.6) and how they satisfy biologically desirable properties: 1) $M$ is nonnegative for all nonnegative $\mathbf{n}, 2) M$ is nondecreasing in $\mathbf{n}, 3) \quad M=0$ if either $m=0$ or $f=0$, and 4) $M$ is homogeneous of degree 1 in $\mathbf{n}$.

We convert the total mating function into the per capita mating rates $U_{m}(\mathbf{n})$ and $U_{f}(\mathbf{n})$ (the average mating rates per available males $m$ or females $f$ respectively),

$$
\begin{aligned}
U_{m}(\mathbf{n}) & =\frac{M(\mathbf{n})}{m} \\
U_{f}(\mathbf{n}) & =\frac{M(\mathbf{n})}{f}
\end{aligned}
$$

so that the total population mating rate is $M=U_{m} m=U_{f} f$.

Many commonly used mating functions are generalized weighted means (Hölder means) of the form

$$
M(\mathbf{n})=\left[\beta f^{\alpha}+(1-\beta) m^{\alpha}\right]^{\frac{1}{\alpha}}
$$

where $\beta$ and $\alpha$ are constants and $0 \leq \beta \leq 1, \alpha<0$ (Haedler 1988; Ianelli et al. 2005, Bessa-Gomes et al. 2010). Figure 2-1 shows several generalized weighted mean mating functions and biologically desirable criteria that they satisfy (McFarland 1972, Pollard 1974, Yellin and Samuelson 1974). If multiple male and female stages interbreed to form different types of unions, stage-specific mating preferences can also be integrated into this mating function (Martcheva and Milner 2001).

It is difficult to distinguish between mating functions using real data, especially in human populations where the sex ratio does not vary significantly (Keyfitz 1972). However, recent empirical studies (Miller and Inouye 2011) support the harmonic mean as a mating function in other species. 
Because the harmonic mean also satisfies a large number of biological criteria and is regarded as one of the most realistic mating functions (Caswell and Weeks 1986, Ianelli et al. 2005), we will use a harmonic mean mating function for our analyses.

\subsubsection{Frequency-dependent dynamics}

The mating process often depends on the relative frequencies, rather than absolute abundances, of males and females. As a result, many mating functions, including the generalized weighted means (2.6), are nonlinear, but homogeneous of degree 1 in $\mathbf{n}$. That is:

$$
M(c \mathbf{n})=c M(\mathbf{n})
$$

for any positive constant $c$.

The per capita mating functions (2.4) and (2.5) are accordingly homogeneous of degree 0 in $\mathbf{n}$, so that:

$$
\begin{gathered}
U_{m}(c \mathbf{n})=U_{m}(\mathbf{n}) \\
U_{f}(c \mathbf{n})=U_{f}(\mathbf{n})
\end{gathered}
$$

If all entries in the projection matrix $\mathbf{A}$ (2.3) are also homogeneous of degree 0 , as they are in our models, the system is frequency-dependent. This means that $\mathbf{A}$ can be written as a function of the population frequency vector:

$$
\mathbf{p}=\frac{\mathbf{n}}{\|\mathbf{n}\|}
$$

where $\|\mathbf{n}\|$ is the 1 -norm of $\mathbf{n}$.

Nonlinear, frequency-dependent models converge asymptotically to an equilibrium population structure $\hat{\mathbf{p}}$ (the stable stage distribution) where all stage frequencies are constant (e.g., see Yellin and Samuelson 1974, Hadeler 1988 for continuous-time models; Caswell and Weeks 1986 for a discrete-time model). The population then grows or decays exponentially at a long-term growth rate given by the dominant eigenvalue $\lambda$ of $\mathbf{A}(\hat{\mathbf{p}})$.

To find the equilibrium stage distribution $\hat{\mathbf{p}}$ and population growth rate $\lambda$, it is sufficient to consider the dynamics of $\mathbf{p}$. It can be shown (Appendix 2.B) that:

$$
\frac{d \mathbf{p}}{d t}=\left(\mathbf{I}_{s}-\mathbf{p} \mathbf{1}^{\top}\right) \mathbf{A} \mathbf{p}
$$

where $\mathbf{I}_{s}$ is a $s \times s$ identity matrix and $\mathbf{1}^{\boldsymbol{\top}}$ is a $1 \times s$ vector of ones. One can integrate (2.10) with a numerical differential equation solver until population frequencies converge to $\hat{\mathbf{p}}$. Then $\lambda$ is the dominant eigenvalue of $\mathbf{A}(\hat{\mathbf{p}})$. Note that the dominant right eigenvector $\mathbf{w}$ of $\mathbf{A}(\hat{\mathbf{p}})$ also equals the stable stage distribution; that is, $\mathbf{w}=\hat{\mathbf{p}}$. 


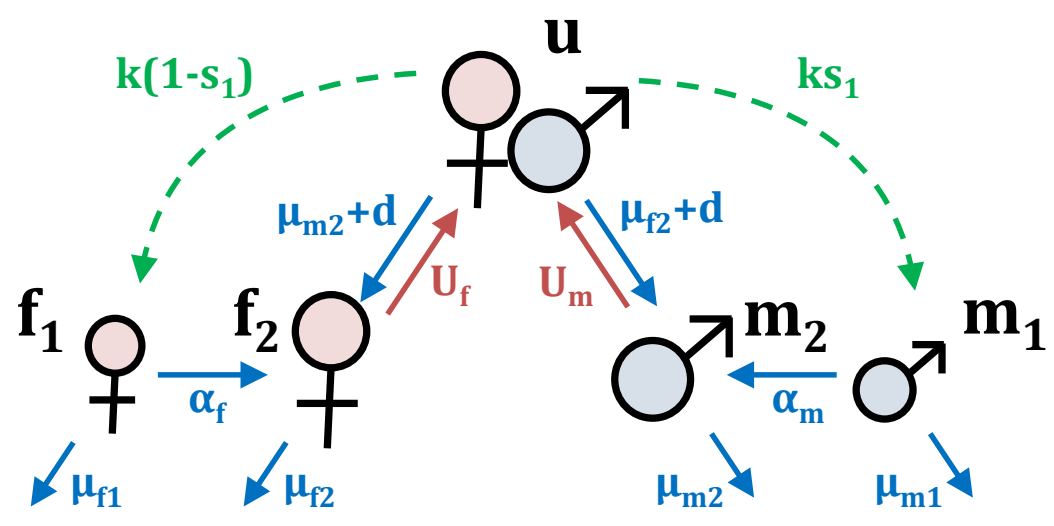

Figure 2-2: Life cycle diagram for a 5 -stage population with juvenile males $m_{1}$ and juvenile females $f_{1}$, adult males $m_{2}$ and adult females $f_{2}$, and reproducing unions $u$. The functions and parameters shown here, as described in Table 2.1, appear in the union formation matrix $\mathbf{U}$ (2.13) (red), birth matrix B (2.14) (green), or transition matrix $\mathbf{T}$ (2.15) (blue).

\subsection{A 5-stage BMMR matrix model}

We now present an example of a BMMR matrix model with five stages: juvenile males $m_{1}$ and juvenile females $f_{1}$, adult males $m_{2}$ and adult females $f_{2}$, and reproducing unions $u$ that consist of one adult male and one adult female. Single adult males and females interact to form unions, which then produce new juvenile offspring (Figure 2-2). A summary of the variables, parameters, and matrices in this model is provided in Table 2.1 .

Similar to (2.1), we write the population vector as

$$
\mathbf{n}(t)=\left(\begin{array}{lllll}
m_{1} & m_{2} & f_{1} & f_{2} & u
\end{array}\right)^{\top}
$$

Assume that only adult males $m_{2}$ and adult females $f_{2}$ mate, and that the total mating rate is given by a harmonic mean mating function. The total and per capita mating functions are thus:

$$
\begin{aligned}
M(\mathbf{n}) & =\frac{2 m_{2} f_{2}}{m_{2}+f_{2}} \\
U_{m}(\mathbf{n}) & =\frac{2 f_{2}}{m_{2}+f_{2}} \\
U_{f}(\mathbf{n}) & =\frac{2 m_{2}}{m_{2}+f_{2}}
\end{aligned}
$$

Again, we consider the life cycle in terms of mating, birth, and transition processes, which are described by matrices $\mathbf{U}, \mathbf{B}$, and $\mathbf{T}$ respectively. 
1. The union formation matrix $\mathbf{U}$ contains the per capita mating functions from (2.12).

$$
\mathbf{U}(\mathbf{n})=\left(\begin{array}{ccccc}
0 & 0 & 0 & 0 & 0 \\
0 & -U_{m}(\mathbf{n}) & 0 & 0 & 0 \\
0 & 0 & 0 & 0 & 0 \\
0 & 0 & 0 & -U_{f}(\mathbf{n}) & 0 \\
0 & \frac{1}{2} U_{m}(\mathbf{n}) & 0 & \frac{1}{2} U_{f}(\mathbf{n}) & 0
\end{array}\right)=\left(\begin{array}{ccccc}
0 & 0 & 0 & 0 & 0 \\
0 & -\frac{M}{m_{2}} & 0 & 0 & 0 \\
0 & 0 & 0 & 0 & 0 \\
0 & 0 & 0 & -\frac{M}{f_{2}} & 0 \\
0 & \frac{M}{2 m_{2}} & 0 & \frac{M}{2 f_{2}} & 0
\end{array}\right)
$$

Note that both $U_{m}$ and $U_{f}$ must halved in the last row of $\mathbf{U}$ to avoid double counting the unions formed from both male and female partners.

2. The birth matrix $\mathbf{B}$ contains $k$, the average reproductive rate of a union, and the primary sex ratio $s_{1}$, the proportion of offspring that are male.

$$
\mathbf{B}=\left(\begin{array}{ccccc}
0 & 0 & 0 & 0 & k s_{1} \\
0 & 0 & 0 & 0 & 0 \\
0 & 0 & 0 & 0 & k\left(1-s_{1}\right) \\
0 & 0 & 0 & 0 & 0 \\
0 & 0 & 0 & 0 & 0
\end{array}\right)
$$

3. The transition matrix $\mathbf{T}$ contains the male mortality rates ( $\mu_{m 1}$ for juveniles, $\mu_{m 2}$ for adults) and female mortality rates ( $\mu_{f 1}$ for juveniles, $\mu_{f 2}$ for adults), the rates of maturation from juveniles to adults ( $\alpha_{m}$ for males, $\alpha_{f}$ for females), and the divorce rate $d$ (rate at which the male-female pair bond breaks). Note that unions may also dissolve due to partner death, and that union dissolution through both death and divorce returns surviving males and females to the single adult stages.

$$
\mathbf{T}=\left(\begin{array}{ccccc}
-\left(\mu_{m 1}+\alpha_{m}\right) & 0 & 0 & 0 & 0 \\
\alpha_{m} & -\mu_{m 2} & 0 & 0 & \mu_{f 2}+d \\
0 & 0 & -\left(\mu_{f 1}+\alpha_{f}\right) & 0 & 0 \\
0 & 0 & \alpha_{f} & -\mu_{f 2} & \mu_{m 2}+d \\
0 & 0 & 0 & 0 & -\left(\mu_{m 2}+\mu_{f 2}+d\right)
\end{array}\right)
$$

As per (2.3), the average of these three matrices is the continuous-time projection matrix $\mathbf{A}(\mathbf{n})$. The corresponding equation for the proportional structure (2.10) is thus:

$$
\frac{d \mathbf{p}}{d t}=\left(\mathbf{I}_{s}-\mathbf{p} \mathbf{1}^{\top}\right) \frac{1}{3}(\mathbf{T}+\mathbf{B}+\mathbf{U}) \mathbf{p}
$$

As shown in Figure 2-3, the population vector $\mathbf{n}$ ultimately grows exponentially, while the frequency vector $\mathbf{p}$ ultimately converges to the constant distribution of stages $\hat{\mathbf{p}}$. To determine the 
a)

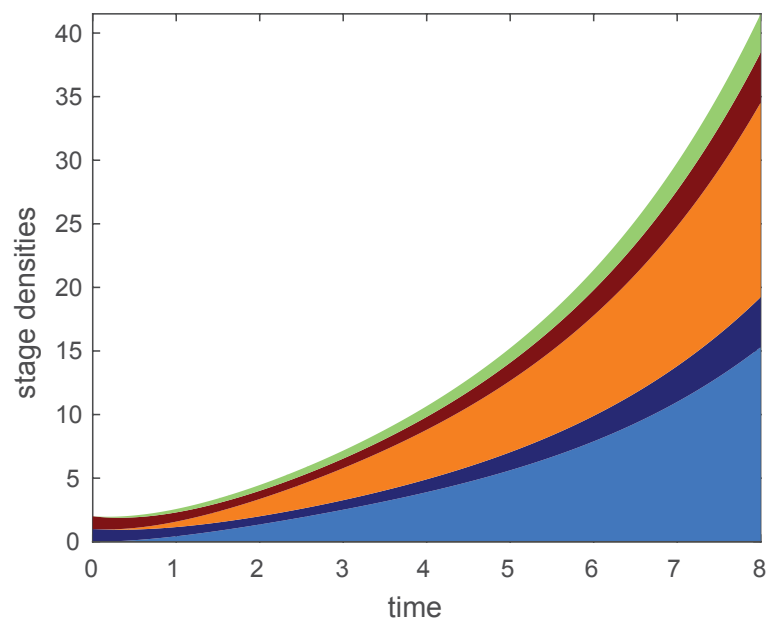

b)

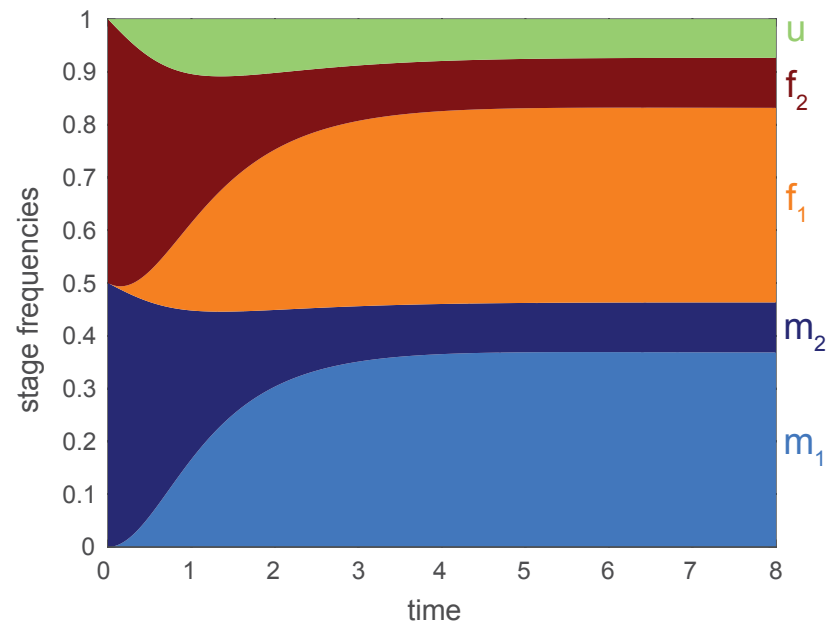

Figure 2-3: Dynamics of the 5-stage BMMR model with monogamous unions and no harvest. The population consists of juvenile males $m_{1}$ and juvenile females $f_{1}$, adult males $m_{2}$ and adult females $f_{2}$, and reproducing unions $u$. a) Growth of the population density vector $\mathbf{n}(2.11)$, where dynamics are given by (2.3). b) Convergence of the population frequency vector $\mathbf{p}(2.9)$, where dynamics are given by (2.16). Parameters are fixed at $\mu_{m 1}=\mu_{f 1}=0.5, \mu_{m 2}=\mu_{f 2}=0.1, \alpha_{m}=\alpha_{f}=0.5, s_{1}=$ $0.5, k=20, d=0.1, h=1, E=0$.

equilibrium stage distribution $\hat{\mathbf{p}}$, we integrated (2.16) with the MATLAB ODE45 differential equation solver until population frequencies converged (e.g., until vector entries do not change significantly over consecutive integration intervals). Sample MATLAB code is provided in the Supplemental Material.

\subsection{Case study: sex-biased harvest in various mating systems}

The mating system determines how males and females organize for breeding and is thus a key component of two-sex population structure (Emlen and Oring 1977). Some species form monogamous, one-to-one pair bonds between males and females (most commonly in birds). Many other species have polygynous mating systems where one male mates with multiple females (e.g., lions, deer, seals), or, more rarely, polyandrous systems where one female mates with multiple males (e.g., jacanas, pipefish). The resulting unions may be transient and limited to a single breeding episode (e.g., lek systems); alternatively, unions may be persistent and last over multiple breeding seasons (e.g., lion harems) or even until partner death (e.g., albatrosses and other species with high mate fidelity) (Cézilly and Danchin 2008).

The role of males and females in the mating system may influence how different species respond to sex-biased harvest. In sport or trophy hunting, harvest is often male-biased and significantly exceeds natural mortality (Festa-Bianchet 2003, Milner 2007). Age or size bias is also common, as larger or older males with well-developed adult characteristics (e.g. large antlers or horns) are usually 


\begin{tabular}{ll}
\hline Matrices and Vectors & \\
\hline $\mathbf{A}$ & projection matrix \\
$\mathbf{B}$ & birth matrix $(2.14)$ \\
$\mathbf{T}$ & transition matrix $(2.15)$ \\
$\mathbf{U}$ & union matrix $(2.13)$ \\
$\mathbf{n}$ & population density vector $(2.11)$ \\
$\mathbf{p}$ & population frequency vector $(2.9)$ \\
$\hat{\mathbf{p}}$ or $\mathbf{w}$ & equilibrium stage structure \\
\hline Population Properties & \\
\hline$\lambda$ & long-term population growth rate, dominant eigenvalue of $\mathbf{A}(\hat{\mathbf{p}})$ \\
$s_{1}$ & primary sex ratio (proportion of offspring that are born male) \\
$s_{2}$ & secondary sex ratio (proportion of adults that are male) \\
$f_{1}, f_{2}$ & juvenile, adult female density \\
$m_{1}, m_{2}$ & juvenile, adult male density \\
$u$ & union (mated pair) density \\
\hline Life Cycle Parameters & \\
\hline$\alpha_{m}, \alpha_{f}$ & male, female maturation rates \\
$d$ & divorce rate (rate at which a male-female pair bond breaks) \\
$h$ & average harem size \\
$k$ & union reproductive rate \\
$\mu_{f 1}, \mu_{f 2}$ & juvenile, adult female mortality rates \\
$\mu_{m}, \mu_{m 2}$ & juvenile, adult male mortality rates \\
$M$ & total mating rate (total unions formed per time) \\
$U_{m}, U_{f}$ & per capita mating rates $(2.4)$ and $(2.5)$ \\
\hline Harvest Parameters & \\
\hline$E$ & total harvest rate in (2.17) \\
$s_{h}$ & harvested sex ratio (proportion of harvest that targets males) in $(2.17)$ \\
\hline
\end{tabular}

Table 2.1: A summary of the variables, parameters, matrices, and population properties in the 5-stage BMMR matrix model.

the most desirable targets. This "unnatural" selection may alter population structure, reproductive strategies, body morphology, and developmental timing (e.g. Ashley 2003, Festa-Bianchet 2003, Allendorf and Hard 2009), with multiple demographic factors, such as age-specific variations in fecundity (Ginsberg 1994), affecting population responses.

These factors motivate the use of a demographic two-sex model in analyzing harvest effects. To this end, we will use our BMMR matrix framework to explore how populations with various mating systems differ in their response to sex-biased harvest. A range of mating systems will be approximated by varying two model parameters, $d$ and $h$ (Table 2.2).

- The divorce rate $d$ is a measure of union transience. Unions with higher values of $d$ are more likely to dissolve after a given mating, while unions with lower values of $d$ are more likely to persist over multiple breeding interactions.

- The harem size $h$ is a measure of polygamy. Unions with $h=1$ are monogamous and consist only of one-to-one male-female pair bonds, while unions with $h>1$ are polygamous groups of size $h+1$. As polyandrous mating systems are relatively rare (Cézilly and Danchin 2008), we will consider only the polygynous form of polygamy, where one male mates with $h$ females. 


\begin{tabular}{|c|c|c|}
\hline & low $d$ (persistent unions) & high $d$ (transient unions) \\
\hline$h=1$ (monogamy) & $\begin{array}{l}\text { persistent pair bonds, high mate } \\
\text { fidelity (e.g., albatross) }\end{array}$ & $\begin{array}{l}\text { serial pair bonds (e.g., } \\
\text { humans, Emperor penguins) }\end{array}$ \\
\hline$h>1$ (polygyny) & $\begin{array}{l}\text { persistent harems (e.g., lion } \\
\text { prides) }\end{array}$ & $\begin{array}{l}\text { leks, scramble competition (e.g., } \\
\text { grouse, cod, horseshoe crabs) }\end{array}$ \\
\hline
\end{tabular}

Table 2.2: Mating systems corresponding to different values of the divorce rate $d$ and harem size $h$.

Harvest strategies are characterized by the total harvest rate $E$ and the harvested sex ratio $s_{h}$ (proportion of the total harvest rate that targets males). Assuming that only adults are harvested, the adult mortality rates are modified by harvest as follows:

$$
\begin{aligned}
\mu_{m 2} & \rightarrow \mu_{m 2}+E s_{h} \\
\mu_{f 2} & \rightarrow \mu_{f 2}+E\left(1-s_{h}\right)
\end{aligned}
$$

To determine how various mating systems, as characterized by different values of $h$ and $d$, respond to sex-biased harvest, we will examine harvest effects on the long-term population growth rate $\lambda$ and the secondary sex ratio $s_{2}$ (proportion of all adults that are male). We will assume that males and females have the same baseline vital rates, and that the primary sex ratio (proportion of males at birth) is 0.5 . Thus, the main sex-specific differences we consider are sex-biased harvest pressures and male versus females roles within the polygynous mating systems.

\subsubsection{Monogamy $(h=1)$}

Consider a monogamous two-sex population with juveniles and adults. The mating process forms unions that are one-to-one pair bonds of adult males and females. This scenario uses the rate matrices $\mathbf{U}, \mathbf{B}$, and $\mathbf{T}$ as given by (2.13), (2.14), and (2.15) respectively, and the mating functions in (2.12). We vary the divorce rate $d$ to explore the effects of transient vs. persistent pair bonds.

As shown in Figure 2-4a, the proportion of adults in the reproductive union stage (mated adults) decreases as $d$ increases. The unharvested population growth rate $\lambda$ similarly decreases (Figure 2-4b), because populations with more transient couples (fewer mated adults) cannot produce offspring as rapidly as populations with more persistent couples (more mated adults). Because males and females have the same baseline vital rates, the secondary sex ratio $s_{2}$ remains unbiased $\left(s_{2}=0.5\right.$, not shown) for all values of $d$.

Figure 2-4c shows how increasingly sex-biased harvest strategies affect population growth. Both biased and unbiased harvest strategies most strongly reduce growth in populations with lower divorce rates, as adult mortality will also disrupt pair bonds. The greatest decreases in $\lambda$ occur when harvest is strongly sex-biased, i.e. $s_{h}$ is close to 0 (only females are harvested) or 1 (only males are harvested). This suggests that monogamous populations with high fidelity pair bonds will be 

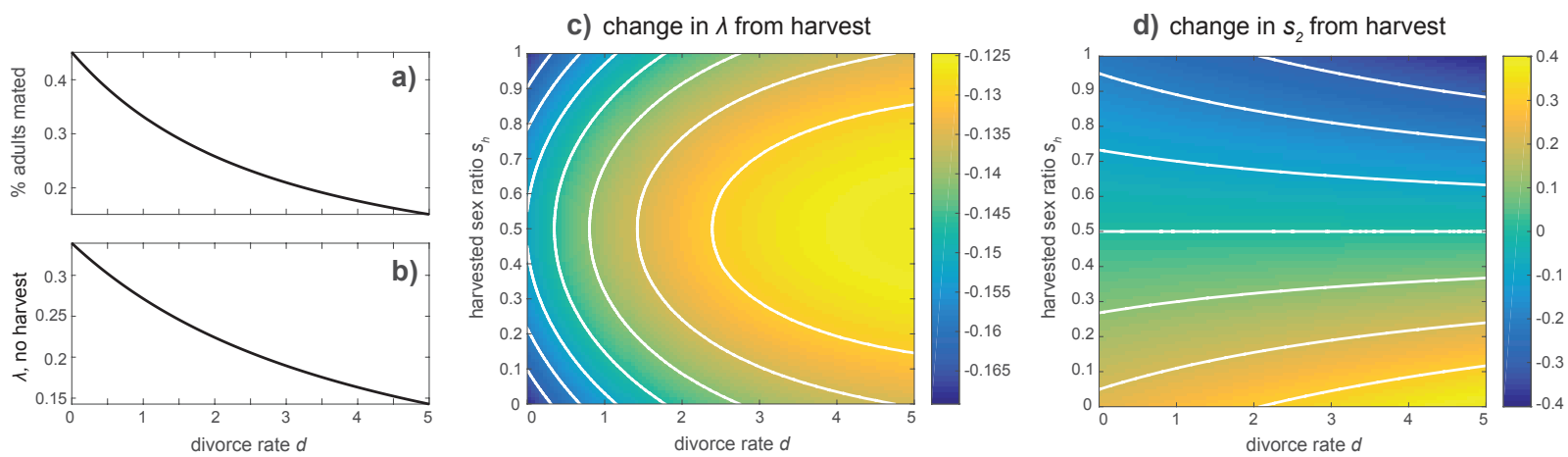

Figure 2-4: Population growth rates, structure, and responses to adult harvest in the monogamous $(h=1)$ model. a) Proportion of mated adults (adults in stage $u$, rather than $m_{2}$ or $f_{2}$ ) for an unharvested population, and the $b$ ) corresponding population growth rates $\lambda$, as functions of the divorce rate $d$. c) The change in $\lambda$ and $d$ ) the change in secondary sex ratio $s_{2}$, when a proportion $s_{h}$ of harvest targets males. Without harvest, $s_{2}=0.5$ for all values of $d$. Other parameters are fixed at $\mu_{m 1}=\mu_{f 1}=0.5, \mu_{m 2}=\mu_{f 2}=0.1, \alpha_{m}=\alpha_{f}=0.5, s_{1}=0.5, k=20, E=1$.

the most impacted by sex-biased harvest, and that concentrating harvest on a single sex will more greatly reduce population growth.

Figure $2-4 \mathrm{~d}$ shows how the same harvest strategies decrease the secondary sex ratio $s_{2}$ from equality. Unsurprisingly, male-biased strategies reduce $s_{2}$, female-biased strategies increase $s_{2}$, and unbiased strategies $\left(s_{h}=0.5\right)$ have relatively minimal effect. Populations with greater divorce rates experience larger reductions in $s_{2}$, possibly due to their lower growth rates (Figure 2-4a) being unable to replenish harvested individuals as rapidly.

\subsubsection{Polygyny $(h>1)$}

Now consider a polygynous population that forms unions consisting of one male with a harem of females. Because the death or departure of a single female changes the harem's size and reproductive rate, we must now account for multiple union (harem) stages.

The stage $u_{i}$ represents polygynous unions consisting of one male and a harem of $i$ females. When $h$ is the maximum harem size, the population vector contains $h$ union stages, which range from a union with a harem of size 1 ( $u_{1}$, equivalent to a monogamous couple) to a union with a harem of size $h\left(u_{h}\right.$, the largest possible union):

$$
\mathbf{n}(t)=\left(\begin{array}{llllllll}
m_{1} & m_{2} & f_{1} & f_{2} & u_{1} & u_{2} & \ldots & u_{h}
\end{array}\right)^{\top}
$$

Assume that when males and females mate, they form the largest possible union $u_{h}$. Their union formation rate is still given by the harmonic mean mating function (2.12), but with the number of 
single females now replaced by the number of prospective harems:

$$
\begin{aligned}
M(\mathbf{n}) & =\frac{2 m_{2} \frac{f_{2}}{h}}{m_{2}+\frac{f_{2}}{h}} \\
U_{m}(\mathbf{n}) & =\frac{2 f_{2}}{h m_{2}+f_{2}} \\
U_{f}(\mathbf{n}) & =\frac{2 m_{2}}{h m_{2}+f_{2}}
\end{aligned}
$$

Note that the total union formation rate $M(\mathbf{n})$ is maximized when the sex ratio of single adults is

$$
\frac{m_{2}}{m_{2}+f_{2}}=\frac{1}{1+\sqrt{h}}
$$

Thus, as the harem size $h$ increases, a higher proportion of single females is needed to maximize the mating rate.

If an individual female has a reproductive rate of $k$, a harem with $i$ females has a total reproductive rate of $i k$; larger harems are thus more productive. Each union $u_{i}$, regardless of harem size, can change in three possible ways (Figure 2-5):

1. The male harem leader dies (with mortality rate $\mu_{m 2}$ ). This returns $i$ adult females to the stage $f_{2}$.

2. A female harem member dies (with mortality rate $\mu_{f 2}$ ). This shrinks the union from $u_{i}$ to $u_{i-1}$. For the union $u_{1}$, which has only one female, $u_{i-1}=u_{0}$ corresponds to the single adult male stage $m_{2}$ (i.e., the death of a wife returns her husband to the pool of unmated singles).

3. A female harem member departs from the union (with divorce rate $d$ ). This returns one female to $f_{2}$ and shrinks the union from $u_{i}$ to $u_{i-1}$. For the union $u_{1}$, divorce dissolves the union and returns one male to $m_{2}$ and one female to $f_{2}$.

As a result, a union may shrink (but not grow) in size due to the departure or death of its members (Figure 2-6). After a union shrinks to the smallest possible size $h=1$, or if the male harem leader dies, the union dissolves and its members return to the stages for unmated adults.

Appendix 2.C shows how to write the rate matrices $\mathbf{U}, \mathbf{B}, \mathbf{T}$ for a polygynous system with maximum harem size $h$. The population vector and matrices for the case where $h=3$ are as 


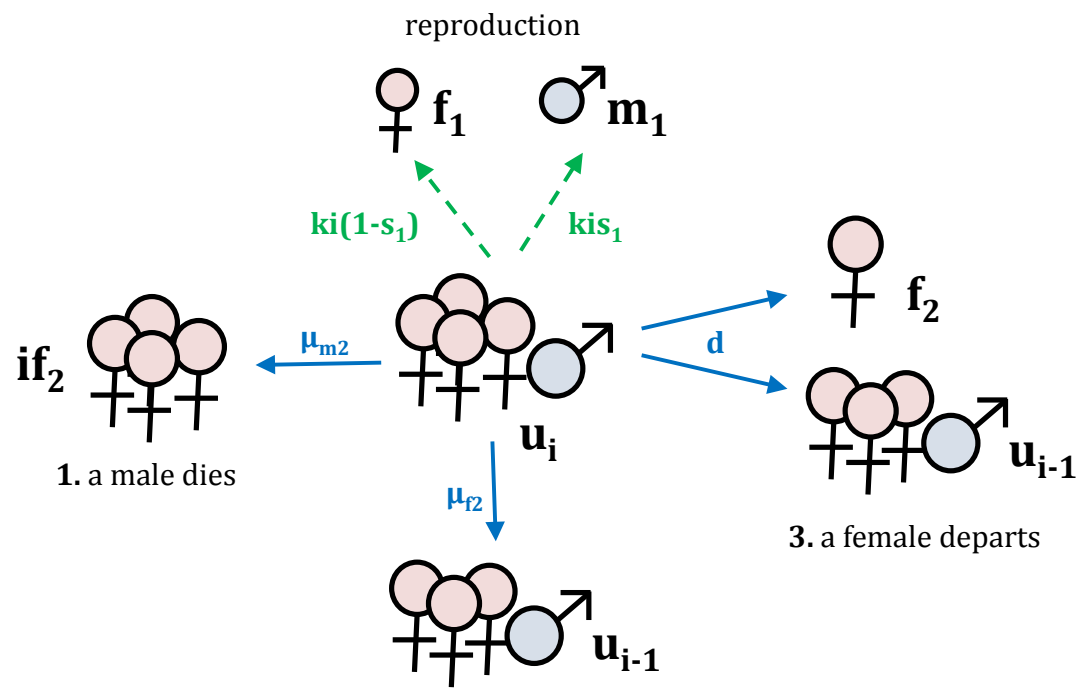

2. a female dies

Figure 2-5: Reproduction and three possible transitions for $u_{i}$, a union with harem size $i$.

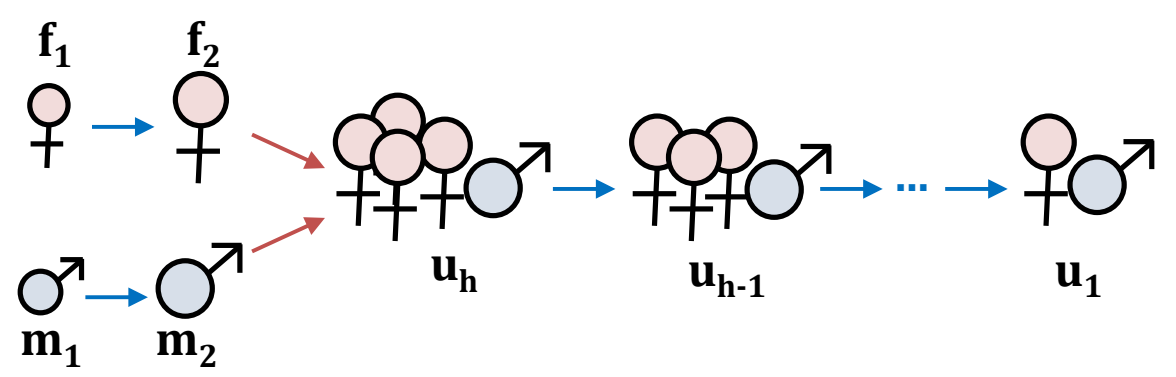

Figure 2-6: Stages in a population with maximum harem size $h$, which include juvenile males $m_{1}$ and juvenile females $f_{1}$, adult males $m_{2}$ and adult females $f_{2}$, and reproducing unions $u_{i}$ (one male with a harem of $i$ females). Adults form harems of size $h$ when mating, and these harems can shrink in size, but not grow, over time. 


\section{c) $\lambda$ before harvest}

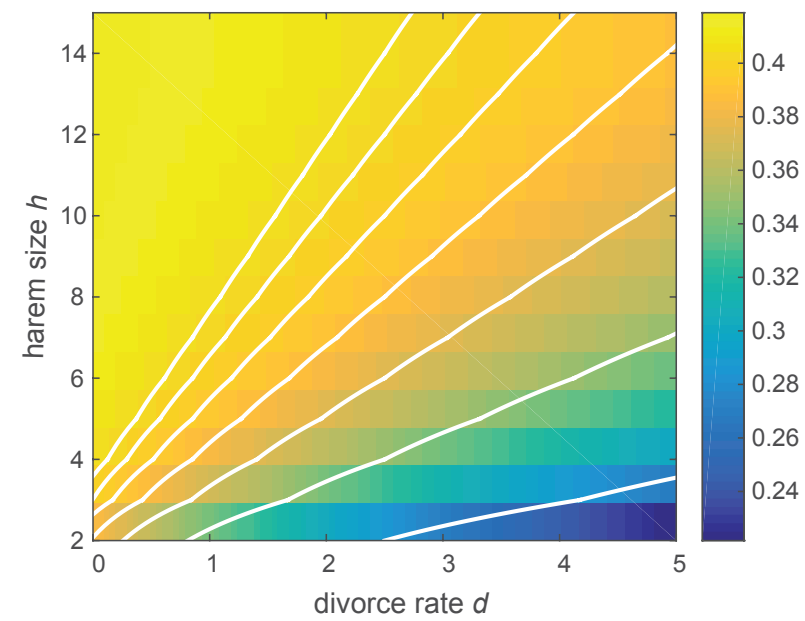

d) $s_{2}$ before harvest

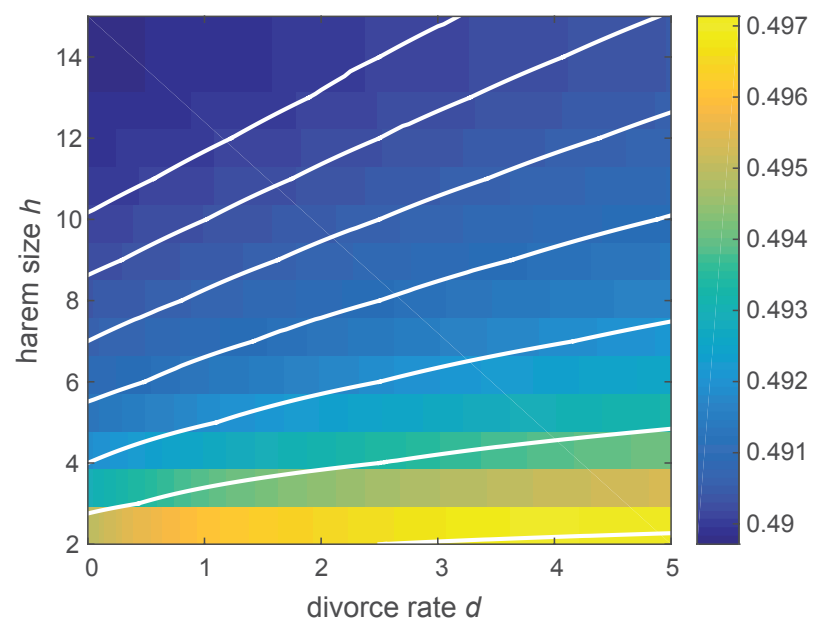

Figure 2-7: Unharvested population dynamics in the polygynous $(h>1)$ union model, as functions of the divorce rate $d$ and harem size $h$. a) Population growth rate $\lambda$. b) Secondary sex ratio $s_{2}$ (proportion of males in the adult population). Other parameters are the same as in Figure 2-4.

follows:

$$
\begin{aligned}
& \mathbf{n}(t)=\left(\begin{array}{lllllll}
m_{1} & m_{2} & f_{1} & f_{2} & u_{1} & u_{2} & u_{3}
\end{array}\right)^{\top} \\
& \mathbf{U}=\left(\begin{array}{ccccccc}
0 & 0 & 0 & 0 & 0 & 0 & 0 \\
0 & -U_{m} & 0 & 0 & 0 & 0 & 0 \\
0 & 0 & 0 & 0 & 0 & 0 & 0 \\
0 & 0 & 0 & -3 U_{f} & 0 & 0 & 0 \\
0 & 0 & 0 & 0 & 0 & 0 & 0 \\
0 & 0 & 0 & 0 & 0 & 0 & 0 \\
0 & \frac{1}{2} U_{m} & 0 & \frac{1}{2} U_{f} & 0 & 0 & 0
\end{array}\right)
\end{aligned}
$$

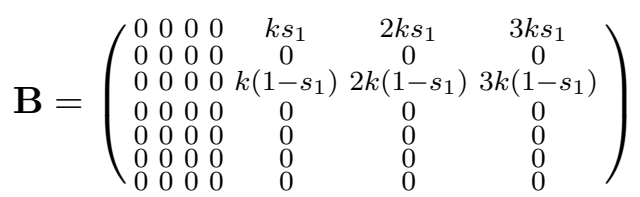

$$
\begin{aligned}
& \mathbf{T}=\left(\begin{array}{ccccccc}
-\left(\mu_{m 1}+\alpha_{m}\right) & 0 & 0 & 0 & 0 & 0 & 0 \\
\alpha_{m} & -\mu_{m 2} & 0 & 0 & \mu_{f 2}+d & 0 & 0 \\
0 & 0 & -\left(\mu_{f 1}+\alpha_{f}\right) & 0 & 0 & 0 & 0 \\
0 & 0 & \alpha_{f} & -\mu_{f 2} & \mu_{m 2}+d & 2 \mu_{m 2}+d & 3 \mu_{m 2}+d \\
0 & 0 & 0 & 0 & -\left(\mu_{m 2}+\mu_{f 2}+d\right) & \mu_{f 2}+d & 0 \\
0 & 0 & 0 & 0 & 0 & -\left(\mu_{m 2}+\mu_{f 2}+d\right) & \mu_{f 2}+d \\
0 & 0 & 0 & 0 & 0 & 0 & -\left(\mu_{m 2}+\mu_{f 2}+d\right)
\end{array}\right)
\end{aligned}
$$

Figure 2-7 shows how the unharvested population growth rate and secondary sex ratio vary across different values of $d$ and $h$. As in the monogamous case, lower divorce rates result in more mated reproducing adults and, thus, higher population growth. Larger harems also have greater population growth, possibly because of their higher total reproductive rates. A union with high $h$ is also more resilient to divorce and female mortality, because it can lose more females before it reaches $h=1$ and dissolves. 
Even without sex-biased harvest, the secondary sex ratio is slightly female-biased $\left(s_{2} \approx 0.494\right)$, but varies only a few tenths of a percentage point across a wide range of $h$ and $d$. Populations with high $h$ and low $d$ (large, persistent harems) are the most biased.

Figure 2-8 demonstrate how female-biased $\left(s_{h}=0\right)$, unbiased $\left(s_{h}=0.5\right)$, and male-biased $\left(s_{h}=1\right)$ harvest strategies affect population growth rates and secondary sex ratios.

1. Female-biased harvest (Figure 2-8a) most strongly reduces growth in populations with large $d$ and small $h$, the same populations with the lowest unharvested growth rates (Figure 2-7a). Smaller harems are less resilient to female-biased harvest for the same reason they are less resilient to divorce and female mortality - because they cannot lose as many females before dissolving.

Female-biased harvest may reduce the average harem size, and also makes it difficult for large harems to form or reform after breaking up. When $h$ is high, a higher proportion of females is needed to maximize the mating rate, in accordance with (2.20), but these females are depleted by harvest. Increasing the divorce rate increases the rate at which harems dissolve. This more drastically reduces growth for larger harems (contours are steeper at large $h$ ), because they need more females to reform.

2. Unbiased harvest (Figure 2-8b) yields similar qualitative trends. Again, populations with higher divorce rates experience greater reductions in growth, and larger harems are more affected by divorce. The effect of increasing divorce is not as pronounced as with female-biased harvest (contours are flatter overall), as less female harvest makes it easier for harems to reform.

At low $h$, however, populations with lower $d$ are actually more impacted by harvest. Low $h$ unions have only a few females and are more likely to dissolve from increased mortality. Unions with high divorce rates are already dissolving quickly, regardless of harvest mortality. Unions with low divorce rates, in contrast, break up much more frequently once harvest mortality occurs. As a result, low $d$, low $h$ populations experience the largest decreases in growth.

3. Male-biased harvest (Figure 2-8c) reverses the effects of increased divorce rate. Focusing harvest on males is more likely to dissolve harems by killing their male leaders. Populations with low $d$ experience the largest reductions in growth, because male-biased harvest makes these unions dissolve more frequently than they normally would (similar to the low $d$, low $h$ case for unbiased harvest).

As in the previous scenarios, the growth of large $h$ populations is less affected by harvest. Even though male-biased mortality causes unions to break up more frequently, it also returns (potentially many) females to the $f_{2}$ pool. This may be beneficial when $h$ is high, as a higher proportion of females is needed to maximize the mating rate in accordance with (2.20). 


\section{change in $\lambda$ from harvest}

\section{change in $s_{2}$ from harvest}

a) female-biased harvest $\left(s_{h}=0\right)$
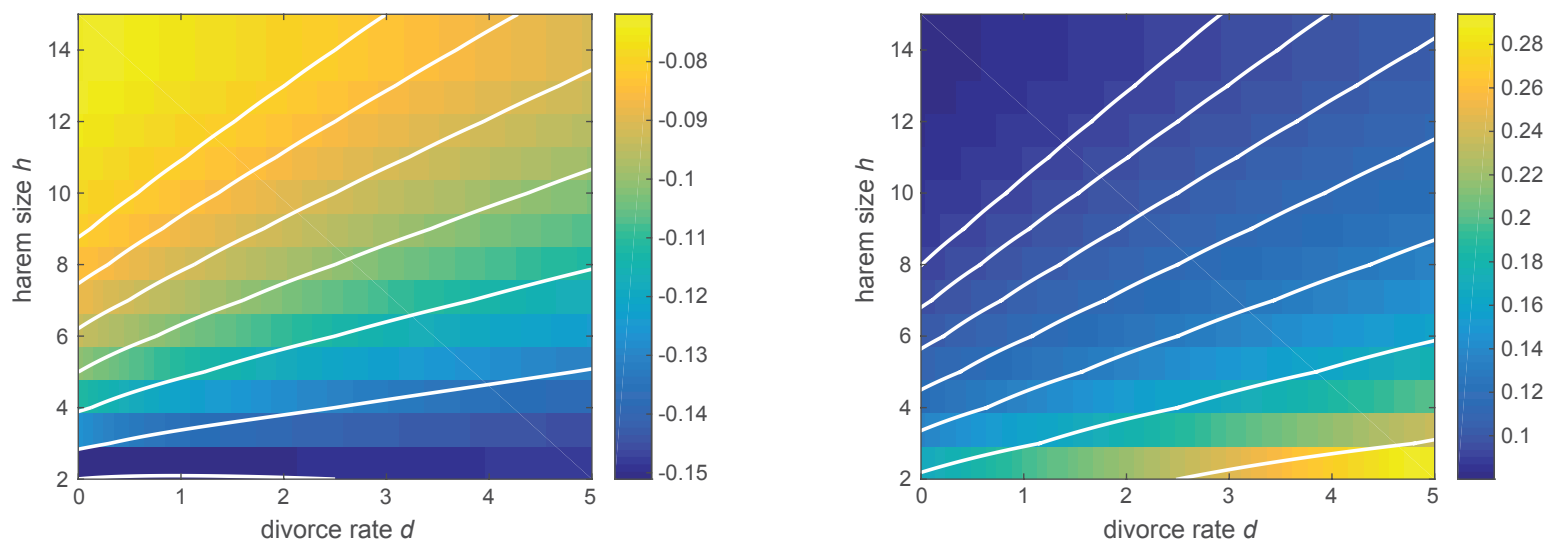

b) unbiased harvest $\left(s_{h}=0.5\right)$
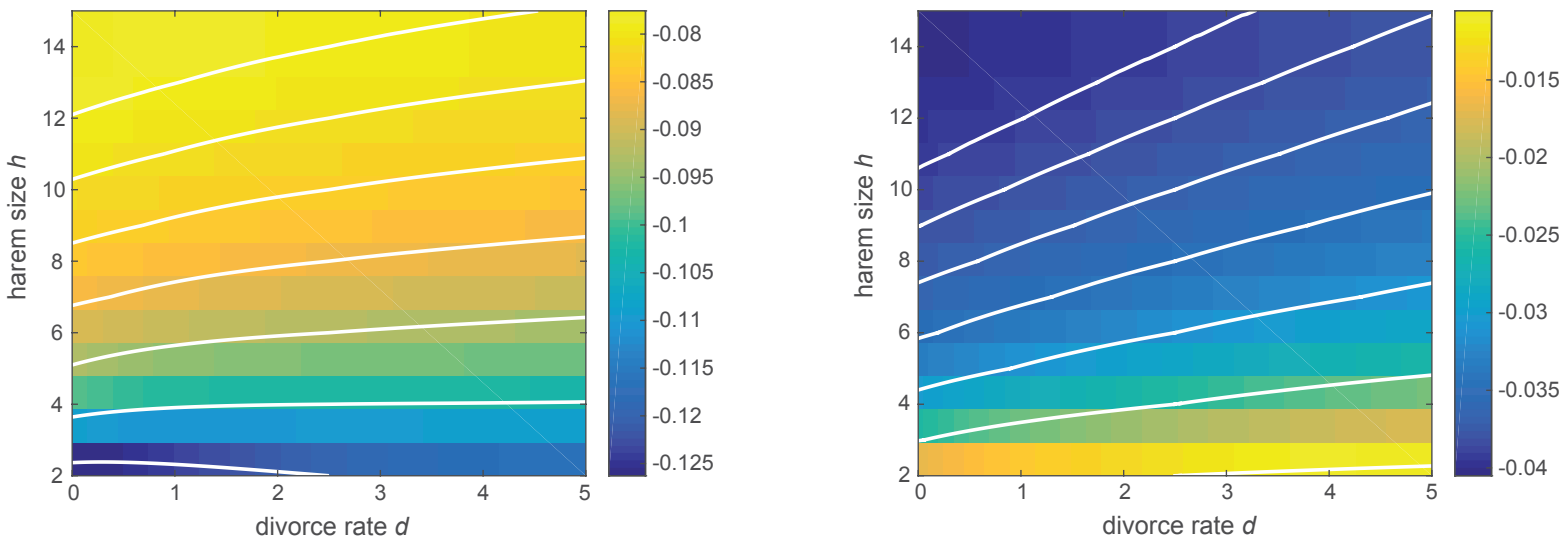

c) male-biased harvest $\left(s_{h}=1\right)$
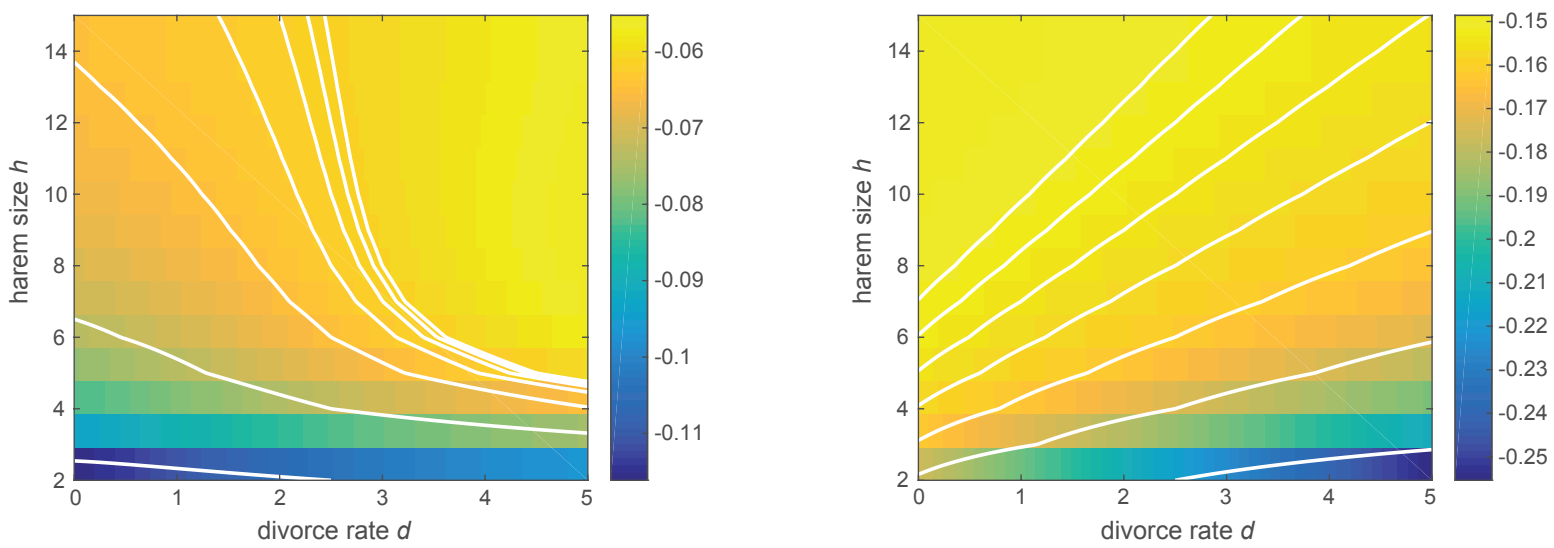

Figure 2-8: Population responses to harvest that is a) female-biased $\left.\left(s_{h}=0\right), b\right)$ unbiased $\left(s_{h}=0.5\right)$, and $c$ ) male-biased $\left(s_{h}=1\right)$ in the polygynous $(h>1)$ model. left) The change in population growth rate $\lambda$. right) The change in secondary sex ratio $s_{2}$. Other parameters are the same as in Figure $2-4$. 

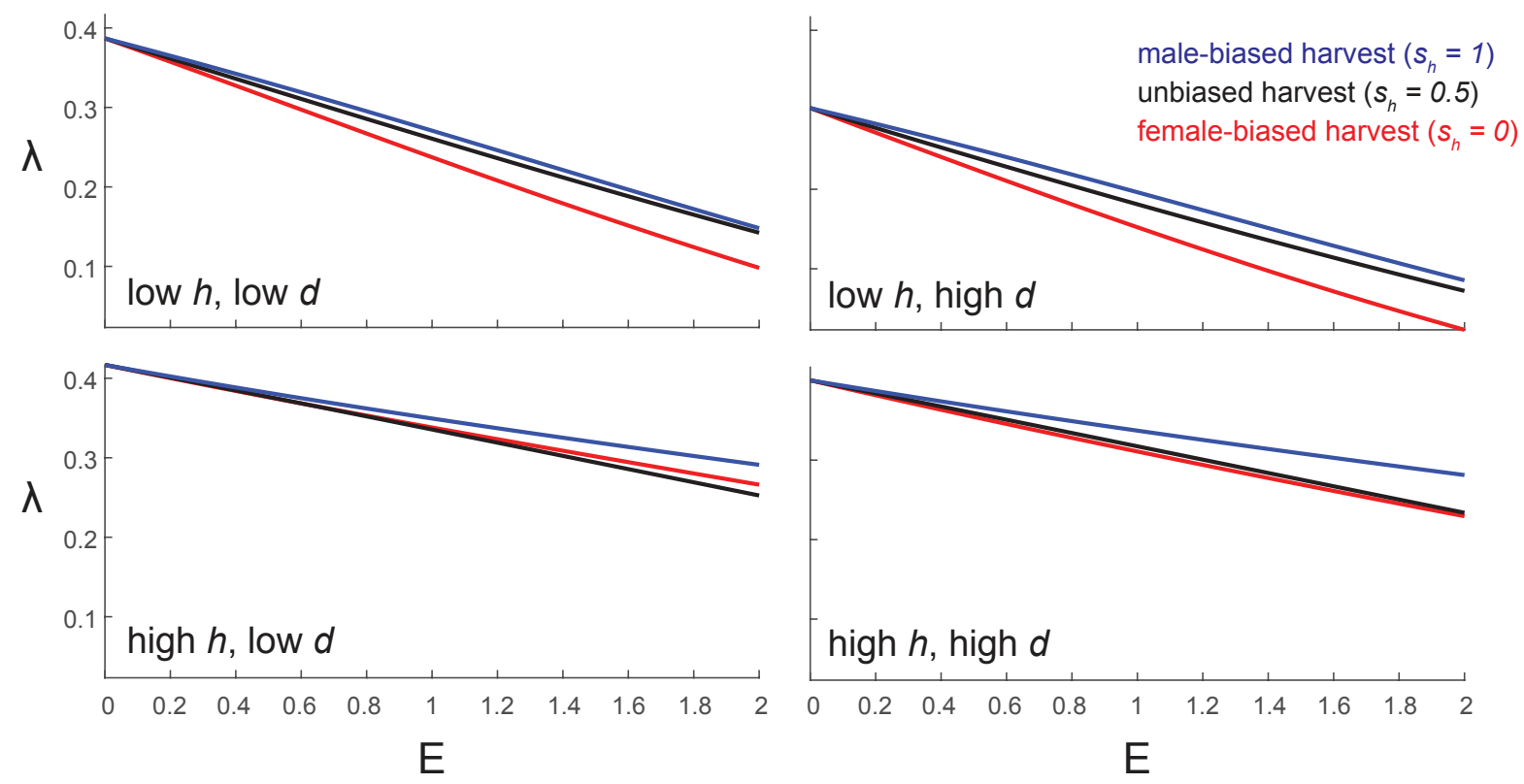

Figure 2-9: Growth rates $\lambda$ as a function of the total harvest rate $E$ for populations with various mating systems. The four types of mating systems shown correspond to different harem sizes $h$ and divorce rates $d$ (Table 2.2); in this example, low $h=2$, high $h=10$, low $d=0$, and high $d=2$. Harvest may be female-biased $\left(s_{h}=0\right)$, unbiased $\left(s_{h}=0.5\right)$, or male-biased $\left(s_{h}=1\right)$. Other parameters are the same as in Figure 2-4.

As expected, the secondary sex ratio $s_{2}$ increases during female-biased harvest, decreases during male-biased harvest, and undergoes only minimal changes when harvest is unbiased (Figure 2-8, right). Populations with high $d$ and low $h$ experience the largest sex ratio shifts under biased harvest. This may be because the smaller growth rates of high $d$, low $h$ populations are less effective in offsetting harvest-induced sex ratio biases.

Figure 2-9 shows how the growth rates of the mating systems in Table 2.2 vary with harvest bias and intensity. Again, we see that high $h$, low $d$ populations (large, persistent harems) have the largest growth rates of all the mating systems, even under harvest. Low $h$, high $d$ populations (small, transient harems) have the smallest growth rates.

Increasing the total harvest rate $E$ in (2.17) amplifies the differences between female-biased, unbiased, and male-biased harvest strategies. Female-biased harvest (red) decreases population growth more severely than male-biased harvest (blue) does, even across populations with different $h$ and $d$. This may be because there is an excess of single males waiting to become harem leaders, whereas single females are usually in shorter supply (especially when $h$ is large). Additionally, the death of a male harem leader immediately dissolves his union; this can return many females to the singles stage, allowing new, full-sized harems to reform with a new male leader. The death of female harem members, in contrast, does not necessarily cause the union to dissolve, and may instead generate small, stunted harems with reduced productivity. 
Depending on the mating system, unbiased harvest (black) can decrease growth rates more or less than female-biased harvest does. In populations with low $h$ (small harems), female-biased harvest has the most drastic impacts of all the harvest strategies - again, perhaps, because small harems cannot afford to lose as many females before dissolving. In populations with high $h$ (large harems), however, unbiased harvest may be just as, if not more, detrimental to population growth.

\subsection{Discussion}

Because different stages and sexes can significantly influence life cycle processes, demographic two-sex models are valuable tools for understanding population dynamics. Based on the BMMR approach, we have developed a more general continuous-time matrix modeling framework that incorporates multiple sexes, mated states, and life cycle processes.

These models can be used to investigate the effects of stage and sex structure, including sex-biased vital rates such as differential mortality or maturation. We have developed one such model to project the long-term dynamics of populations with various mating systems and their potential responses to sex-biased harvest. Our results suggest that multiple mating factors, including both harem size and persistence, affect not only unharvested population growth, but also the responses of growth rate and sex ratio to sex-biased harvest.

In unharvested populations, large divorce rates (more transient unions) tend to reduce population growth, especially when harem size is small. Sex-biased harvest affects not only population sex ratios, but also long-term growth rates, with effects depending on sex bias, harem size, and divorce rate. These complex, and sometimes counterintuitive, nuances would be impossible to capture without a demographic two-sex model like this, motivating the use of such models in ecological studies.

Our two-axis depiction of various mating systems is, of course, a simplification. While we have chosen to focus specifically on the effects of harem size and persistence, there are many factors that distinguish different breeding and offspring production strategies. Many mating systems, for example, differ in terms of the parental investment made by males and females; while polygynous males tend to provide minimal parental care, monogamous males invest on par with their female partners (Emlen and Oring 1977, Cézilly and Danchin 2008). As a result, harvesting a particular sex in different mating systems may have very different consequences for offspring survival. Other species have additional nuances in how they respond to sex-biased harvest - African lions, for instance, commit infanticide when male harem leaders are killed (Whitman et al. 2014), which exacerbates the effects of male harvest on population growth.

How populations respond to selective harvest strategies also has important consequences for evolution. Growing evidence suggests that evolutionary considerations are relevant to sustainable long-term management (Ashley 2003), and that human-induced selection is especially important for harvested species. As harvest mortalities are often more severe and selective than natural 
mortalities, they may drive evolution in directions that would not occur under natural conditions. The evolution of mating strategies or sex ratios, for example, would affect population responses to continued harvest pressure. Ideally, sex ratios would evolve to favor the sex most depleted by harvest, allowing populations to compensate for harvest-induced mortalities in the long run. However, there are many factors, including population structure, the timing of mortality, and sex-biased offspring costs, that modulate sex ratio evolution (Shyu and Caswell, in preparation).

\subsubsection{BMMR matrix models in discrete time}

While all our BMMR matrix models thus far have been formulated in continuous time, a discrete-time approach may be better suited for cases where life cycle processes do not occur simultaneously, e.g. when there is seasonal separation between breeding, mortality, and other periodic events.

In a discrete-time matrix model, the product of the population vector $\mathbf{n}(t)$ and a projection matrix $\mathbf{A}_{d}$ produces a series of coupled difference equations that project the population forward from time $t$, e.g.:

$$
\mathbf{n}(t+1)=\mathbf{A}_{d} \mathbf{n}(t)
$$

The entries in $\mathbf{A}_{d}$ are either transition probabilities, which are bounded between 0 and 1 , or the per capita number of offspring produced by each stage.

Let the matrices $\mathbf{U}_{d}, \mathbf{B}_{d}$, and $\mathbf{T}_{d}$ describe stage-specific transition probabilities in consecutive mating, birth, and transition periods of length $\Delta t$. The total projection matrix $\mathbf{A}_{d}$, which projects the population over all three periods, is the product of the three periodic matrices (Caswell 2001, Chapter 13):

$$
\begin{aligned}
\mathbf{n}(t+3 \Delta t) & =\mathbf{T}_{d} \mathbf{B}_{d} \mathbf{U}_{d} \mathbf{n}(t) \\
& =\mathbf{A}_{d} \mathbf{n}(t)
\end{aligned}
$$

Example matrices for a 5-stage, discrete-time BMMR model are as follows. The mating matrix $\mathbf{U}_{d}$ can be written as:

$$
\mathbf{U}_{d}=\left(\begin{array}{ccccc}
1 & 0 & 0 & 0 & 0 \\
0 & 1-M_{m} & 0 & 0 & 0 \\
0 & 0 & 1 & 0 & 0 \\
0 & 0 & 0 & 1-M_{f} & 0 \\
0 & \frac{1}{2} M_{m} & 0 & \frac{1}{2} M_{f} & 1
\end{array}\right)
$$

where $M_{m}$ and $M_{f}$ are per capita male and female mating probabilities. 
The birth matrix $\mathbf{B}_{d}$ can be written as:

$$
\mathbf{B}_{d}=\left(\begin{array}{ccccc}
1 & 0 & 0 & 0 & L s_{1} \\
0 & 1 & 0 & 0 & 0 \\
0 & 0 & 1 & 0 & L\left(1-s_{1}\right) \\
0 & 0 & 0 & 1 & 0 \\
0 & 0 & 0 & 0 & 1
\end{array}\right)
$$

where $\mathrm{L}$ is the average litter size (number of offspring produced per couple).

And the transition matrix $\mathbf{T}_{d}$ can be written as:

$$
\mathbf{T}_{d}=\left(\begin{array}{ccccc}
p_{m 1} & 0 & 0 & 0 & 0 \\
a_{m} & p_{m 2} & 0 & 0 & \sigma p_{m 2}+(1-\sigma)\left(1-p_{f 2}\right) p_{m 2} \\
0 & 0 & p_{f 1} & 0 & 0 \\
0 & 0 & a_{f} & p_{f 2} & \sigma p_{f 2}+(1-\sigma)\left(1-p_{m 2}\right) p_{f 2} \\
0 & 0 & 0 & 0 & (1-\sigma) p_{m 2} p_{f 2}
\end{array}\right)
$$

where the $p_{i}$ are the probabilities that an individual of stage $i$ survives over the time interval, $a_{m}$ and $a_{f}$ are male and female maturation probabilities, and $\sigma$ is the probability of divorce.

Our preliminary results for these 5-stage, BMMR-based models suggest that the qualitative outcomes of the continuous-time and discrete-time approaches are often similar. However, each approach has advantages and disadvantages that will determine which problems it is best suited for. In discrete time, for instance, it is possible to have a transition of $100 \%$ probability (e.g., all juveniles becoming adults after a certain period). This is not as straightforward in the continuous-time model, as it would require either an infinite transition rate or a periodic "pulse" of transitions after some integration interval.

In the discrete-time model, however, all transition probabilities must be bounded between 0 and 1. This sets constraints on the union formation matrix $\mathbf{U}_{d}$ in (2.27), for example, in that the probability of a male or female transitioning into the union stage ( $M_{m}$ and $M_{f}$ respectively) cannot be greater than 1 . This is problematic when the number of unions is greater than either the number of males or females, as in promiscuous mating systems where individuals can form multiple pair bonds. The continuous-time union matrix (2.13) allows unbounded union formation rates that better accommodate such systems.

In discrete time, all possible transitions from a given stage must also have a total probability of 1 , which complicates the transition matrix $\mathbf{T}_{d}$. Compare, for example, the continuous-time rate matrix in (2.15) to the corresponding discrete-time probability matrix in (2.29). The discrete-time matrix is more complex because it must account for all possible union fates, including multiple deaths and divorce over a given time interval. The continuous-time transition matrix (2.15) is comparably simpler, as multiple events cannot occur simultaneously on a strictly continuous timescale. 
Alternatively, rate matrices from the continuous-time formulation can be transformed into discrete time using the matrix exponential, as in (2.31) and (2.32). Another discrete-time projection matrix can thus be written as the product of three matrix exponentials.

Noting that $\mathbf{n}(t+\Delta t)=e^{\mathbf{A}_{c} \Delta t} \mathbf{n}(t)$, the matrices within the matrix exponentials can also be multiplied by scaling constants that correspond to the relative lengths of the mating, birth, and transition periods ( $a, b$, and $c$ respectively):

$$
\mathbf{n}(t+a+b+c)=\frac{e^{a \mathbf{T}} e^{b \mathbf{B}} e^{c \mathbf{U}}}{a+b+c} \mathbf{n}(t)
$$

Although this approach combines useful elements from both the continuous-time and discrete-time formulations, calculating matrix exponentials is often computationally intensive. Differentiating the eigenvalues of (2.30) for sensitivity analysis also requires the derivatives of matrix exponentials, which are more difficult to compute.

\subsection{Acknowledgments}

This work was supported by a National Science Foundation Graduate Research Fellowship under

Grant 1122374, NSF Grants DEB1145017 and DEB1257545 (to H. Caswell), Advanced Grant 322989 from the European Research Council (to H. Caswell), and the Woods Hole Oceanographic Institution Academic Programs Office.

\section{Bibliography}

[1] Allendorf FW, Hard JJ. 2009. Human-induced evolution caused by unnatural selection through harvest of wild animals. Proceedings of the National Academy of Sciences. 106: 9987-9994.

[2] Ashley MV, Willson MF, Pergams ORW, O’Dowd DJ, Gende SM, Brown JS. 2003. Evolutionarily-enlightened management. Biological Conservation. 111: 115-123.

[3] Bessa-Gomes C, Legendre S, Clobert J. 2010. Discrete two-sex models of population dynamics: On modeling the mating function. Acta Oecologica. 36: 439-445.

[4] Caswell H, Weeks DE. 1986. Two-sex models: chaos, extinction, and other dynamic consequences of sex. American Naturalist. 128: 707-735.

[5] Caswell H. 2001. Matrix population models. Sunderland: Sinauer Associates, Inc.

[6] Caswell H. 2008. Perturbation analysis of nonlinear matrix models. Demographic Research. 18: 59-116.

[7] Cézilly F, Danchin É. 2008. Mating Systems and Parental Care. In: Behavioural Ecology, Danchin É, Girladeau LA, Cézilly F. (eds). Oxford University Press: Oxford, 429-465.

[8] Emlen ST, Oring LW. 1977. Ecology, sexual selection, and the evolution of mating systems. Science. 197: 215-223. 
[9] Festa-Bianchet M. 2003. Exploitative wildlife management as a selective pressure for life-history evolution of large mammals. In Animal Behavior and Wildlife Conservation (eds Festa-Bianchet M, Apollonio M). Washington, D.C.: Island Press. pp. 191-210.

[10] Fredrickson A. 1971. A mathematical theory of age structure in sexual populations: random mating and monogamous marriage models. Mathematical Biosciences. 20: 117-143.

[11] Ginsberg JS, Milner-Gulland EJ. 1994. Sex-based harvesting and population dynamics in ungulates: implications for conservation and sustainable use. Conservation Biology. 18: 157-166.

[12] Hadeler KP. 1989. Pair formation in age-structured populations. Acta Applicandae Mathematicae. 14: 91-102.

[13] Hadeler KP. 1993. Pair formation with maturation period. Journal of Mathematical Biology. 32: $1-15$.

[14] Hadeler KP, Waldstätter R, Wörz-Busekros A. 1988. Models for pair formation in bisexual populations. Journal of Mathematical Biology. 26: 635-649.

[15] Hoppensteadt F. 1975. Mathematical theories of populations: demographics, genetics, and epidemics. SIAM: Philadelphia.

[16] Iannelli, M, Martcheva M, Milner F. 2005. Gender-structured population modeling: mathematical methods, numerics, and simulations. Philadelphia: Society for Industrial and Applied Mathematics.

[17] Jenouvrier S, Caswell H, Barbraud C, Weimerskirch H. 2010. Mating behavior, population growth, and the operational sex ratio: a periodic two-sex model approach. The American Naturalist. 175:739-752.

[18] Karmel PH. 1974. The relations between male and female reproduction rates. Population Studies. 1: 249-274.

[19] Kendall DG. 1949. Stochastic processes and population growth. Journal of the Royal Statistical Society: Series B. 11: 230-264.

[20] Keyfitz N. 1972. The mathematics of sex and marriage. Proceedings of the Sixth Berkeley Symposium on Mathematical Statistics and Probability. 4: 89-108.

[21] Kuczynski RR. 1932. Fertility and reproduction. Falcon Press: New York.

[22] Martcheva M, Milner F. 2001. The mathematics of sex and marriage, revisited. Mathematical Population Studies. 9: 123-141.

[23] McFarland DD. 1972. Comparison of alternative marriage models. In Population Dynamics (ed Greville TNE). New York: Academic Press. pp. 89-106.

[24] Miller TEX, Inouye BD. 2011. Confronting two-sex demographic models with data. Ecology. 92: 2141-2151.

[25] Miller TEX, Shaw AK, Inouye BD, Neubert MG. 2011. Sex-biased dispersal and the speed of two-sex invasions. The American Naturalist. 177: 549-561. 
[26] Pollak RA. 1986. A reformulation of the two-sex problem. Demography. 23: 247-259.

[27] Pollak RA. 1987. The two-sex problem with persistent unions: a generalization of the birth matrix-mating rule model. Theoretical Population Biology. 32: 176-187.

[28] Pollak RA. 1990. Two-sex demographic models. Journal of Political Economy. 98: 399-420.

[29] Pollard AH. 1948. The measurement of reproductivity. Journal of the Institute of Actuaries. 74: $288-318$.

[30] Pollard JH. 1974. The two-sex problem. In Mathematical models for the growth of human populations. Cambridge: Cambridge University Press.

[31] Rankin DJ, Kokko H. 2007. Do males matter? The role of males in population dynamics. Oikos. 116: 335-348.

[32] Sundelöf A, Åberg P. 2006. Birth functions in stage structured two-sex models. Ecological Modelling. 193: 787-795.

[33] Shyu E, Caswell H. A demographic model for sex ratio evolution and the effects of sex-biased offspring costs. In preparation.

[34] Whitman K, Starfield AM, Quadling HS, Packer C. 2004. Sustainable trophy hunting of African lions. Nature. 428: 175-177.

[35] Yellin J, Samuelson PA. 1974. A dynamical model for human population. Proceedings of the National Academy of Sciences. 71: 2813-2817. 


\section{Chapter 2 Appendix}

\section{A Averaging transition rate matrices}

We show that the continuous-time projection matrix $\mathbf{A}$ is the average of the individual transition rate matrices in (2.3) as follows. First note that a continuous-time system can be integrated using the matrix exponential.

$$
\frac{d \mathbf{n}}{d t}=\mathbf{A n}(t) \quad \rightarrow \quad \mathbf{n}(t)=e^{\mathbf{A} t} \mathbf{n}(0)
$$

Thus, the matrix exponential $e^{\Delta t \mathbf{A}}$ projects the population over a discrete period of length $\Delta t$ :

$$
\mathbf{n}(t+\Delta t)=e^{\Delta t \mathbf{A}} \mathbf{n}(t)
$$

and the population can be projected over multiple life cycle periods of length $\Delta t$ using a periodic matrix product (Caswell 2001, Chapter 13):

$$
\mathbf{n}(t+3 \Delta t)=e^{\Delta t \mathbf{T}} e^{\Delta t \mathbf{B}} e^{\Delta t \mathbf{U}} \mathbf{n}(t)
$$

As $\Delta t \rightarrow 0$ in the limit of continuity,

$$
\begin{aligned}
\mathbf{n}(t+3 \Delta t) & \approx(1+\Delta t \mathbf{T})(1+\Delta t \mathbf{B})(1+\Delta t \mathbf{U}) \mathbf{n}(t) \\
& \approx[1+\Delta t(\mathbf{T}+\mathbf{B}+\mathbf{U})] \mathbf{n}(t)
\end{aligned}
$$

By the limit definition,

$$
\begin{aligned}
\frac{d \mathbf{n}}{d t} & =\lim _{\Delta t \rightarrow 0} \frac{\mathbf{n}(t+3 \Delta t)-\mathbf{n}(t)}{3 \Delta t} \\
& =\frac{1}{3}(\mathbf{T}+\mathbf{B}+\mathbf{U}) \mathbf{n}(t) \\
& =\mathbf{A n}(t)
\end{aligned}
$$

as in (2.3). 


\section{B Derivatives of a frequency vector}

Given the population vector of stage frequencies

$$
\mathbf{p}=\frac{\mathbf{n}}{\|\mathbf{n}\|}
$$

the derivative $\frac{d \mathbf{p}}{d t}$ is

$$
\begin{aligned}
\frac{d \mathbf{p}}{d t} & =\frac{\|\mathbf{n}\| \frac{d \mathbf{n}}{d t}-\mathbf{n} \frac{d\|\mathbf{n}\|}{d t}}{\|\mathbf{n}\|^{2}} \\
& =\frac{1}{\|\mathbf{n}\|} \frac{d \mathbf{n}}{d t}-\frac{\mathbf{n}}{\|\mathbf{n}\|^{2}} \frac{d\|\mathbf{n}\|}{d t}
\end{aligned}
$$

Let $\mathbf{1}^{\top}$ be a $1 \times s$ vector of ones. Because $\mathbf{n}$ is a positive vector,

$$
\begin{gathered}
\|\mathbf{n}\|=\mathbf{1}^{\top} \mathbf{n} \\
\frac{d\|\mathbf{n}\|}{d t}=\mathbf{1}^{\top} \frac{d \mathbf{n}}{d t}
\end{gathered}
$$

Then (2.37) can be rewritten as

$$
\begin{aligned}
\frac{d \mathbf{p}}{d t} & =\frac{1}{\mathbf{1}^{\top} \mathbf{n}} \frac{d \mathbf{n}}{d t}-\frac{\mathbf{n}}{\left(\mathbf{1}^{\top} \mathbf{n}\right)^{2}} \mathbf{1}^{\top} \frac{d \mathbf{n}}{d t} \\
& =\frac{1}{\mathbf{1}^{\top} \mathbf{n}}\left(\mathbf{I}_{s}-\mathbf{p} \mathbf{I}^{\top}\right) \frac{d \mathbf{n}}{d t} \\
& =\frac{1}{\mathbf{1}^{\top} \mathbf{n}}\left(\mathbf{I}_{s}-\mathbf{p} \mathbf{I}^{\top}\right) \mathbf{A} \mathbf{n}(t)
\end{aligned}
$$

where $\mathbf{I}_{s}$ is a $s \times s$ identity matrix.

If the population is initialized with a population vector $\mathbf{n}=\mathbf{p}$ so that $\|\mathbf{n}\|=\mathbf{1}^{\top} \mathbf{n}=1$, then (2.39) can be rewritten as:

$$
\frac{d \mathbf{p}}{d t}=\left(\mathbf{I}_{s}-\mathbf{p 1}^{\top}\right) \mathbf{A} \mathbf{p}(t)
$$

as in $(2.10)$.

\section{C BMMR matrices for a polygynous system}

Consider a polygynous system with a maximum harem size of $h$. When $h$ is large, it is cumbersome to write the rate matrices $\mathbf{U}, \mathbf{B}, \mathbf{T}$ in full, especially since many of their entries will be zeros. Instead, we will consider these matrices in terms of their contributions to these nine regions of the 
projection matrix:

$$
\mathbf{A}=\left(\begin{array}{c|c|c}
\mathbf{A}_{m \rightarrow m} & \mathbf{A}_{f \rightarrow m} & \mathbf{A}_{u \rightarrow m} \\
\hline \mathbf{A}_{m \rightarrow f} & \mathbf{A}_{f \rightarrow f} & \mathbf{A}_{u \rightarrow f} \\
\hline \mathbf{A}_{m \rightarrow u} & \mathbf{A}_{f \rightarrow u} & \mathbf{A}_{u \rightarrow u}
\end{array}\right)
$$

For the union formation matrix $\mathbf{U}$, the only regions with nonzero contributions are:

$$
\begin{aligned}
& \mathbf{U}_{m \rightarrow m}=\left(\begin{array}{cc}
0 & 0 \\
0 & -U_{m}
\end{array}\right) \quad 2 \times 2 \\
& \mathbf{U}_{f \rightarrow f}=\left(\begin{array}{cc}
0 & 0 \\
0 & -h U_{f}
\end{array}\right) \quad 2 \times 2 \\
& \mathbf{U}_{m \rightarrow u}=\left(\begin{array}{cc}
0 & 0 \\
& \vdots \\
0 & -\frac{1}{2} U_{m}
\end{array}\right) \quad h \times 2 \\
& \mathbf{U}_{f \rightarrow u}=\left(\begin{array}{cc}
0 & 0 \\
& \vdots \\
0 & -\frac{1}{2} U_{f}
\end{array}\right) \quad h \times 2
\end{aligned}
$$

For the birth matrix $\mathbf{B}$, the only regions with nonzero contributions are:

$$
\begin{aligned}
& \mathbf{B}_{u \rightarrow m}=\left(\begin{array}{cccc}
k s_{1} & 2 k s_{1} & \ldots & k h s_{1} \\
0 & 0 & \ldots & 0
\end{array}\right) \quad 2 \times h \\
& \mathbf{B}_{u \rightarrow f}=\left(\begin{array}{cccc}
k\left(1-s_{1}\right) & 2 k\left(1-s_{1}\right) & \ldots & k h\left(1-s_{1}\right) \\
0 & 0 & \ldots & 0
\end{array}\right) 2 \times h
\end{aligned}
$$

For the transition matrix $\mathbf{T}$, the only regions with nonzero contributions are:

$$
\begin{aligned}
& \mathbf{T}_{m \rightarrow m}=\left(\begin{array}{cc}
-\left(\mu_{m 1}+\alpha_{m}\right) & 0 \\
\alpha_{m} & -\mu_{m 2}
\end{array}\right) \quad 2 \times 2 \\
& \mathbf{T}_{f \rightarrow f}=\left(\begin{array}{cc}
-\left(\mu_{f 1}+\alpha_{f}\right) & 0 \\
\alpha_{f} & -\mu_{f 2}
\end{array}\right) \quad 2 \times 2 \\
& \mathbf{T}_{u \rightarrow m}=\left(\begin{array}{cccc}
0 & 0 & \ldots & 0 \\
\mu_{f 2}+d & 0 & \ldots & 0
\end{array}\right) \quad 2 \times h \\
& \mathbf{T}_{u \rightarrow f}=\left(\begin{array}{cccc}
0 & 0 & \ldots & 0 \\
\mu_{m 2}+d & 2 \mu_{m 2}+d & \ldots & h \mu_{m 2}+d
\end{array}\right) 2 \times h
\end{aligned}
$$

The $h \times h$ submatrix $\mathbf{T}_{u \rightarrow u}$ is also nonzero. It contains entries of $-\left(\mu_{m 2}+\mu_{f 2}+d\right)$ all along its diagonal, and entries of $\mu_{f 2}+d$ all along its first superdiagonal. 
As an example, the $\mathbf{U}, \mathbf{B}, \mathbf{T}$ matrices in the case where $h=3$ are provided in $(2.22),(2.23)$, and (2.24) respectively. 


\section{Chapter 3}

\section{A demographic model for sex ratio evolution and the effects of sex-biased offspring costs}

\subsection{Abstract}

The evolution of the primary sex ratio, the proportion of male births in an individual's offspring production strategy, is a frequency-dependent process that selects against the more common sex. Because reproduction is shaped by the entire life cycle, sex ratio theory would benefit from explicitly two-sex models that include some form of life cycle structure.

We present a demographic approach to sex ratio evolution that combines adaptive dynamics with nonlinear matrix population models. We also determine the evolutionary and convergence stability of singular strategies using matrix calculus. These methods allow the incorporation of any population structure, including multiple sexes and stages, into evolutionary projections.

Using this framework, we compare how four different interpretations of sex-biased offspring costs affect sex ratio evolution. We find that demographic differences affect evolutionary outcomes and that, contrary to prior belief, sex-biased mortality after parental investment can bias the primary sex ratio (but not the corresponding reproductive value ratio).

Keywords: sex ratio evolution, adaptive dynamics, two-sex models, matrix population models, evolutionarily singular strategies, offspring costs, reproductive value

\subsection{Introduction}

Sex ratio evolution is the one of the oldest life history questions and a well-known example of frequency-dependent selection. Although the primary sex ratio $s_{1}$ (proportion of offspring that are born male) is nearly equal in many mammals, including humans, sex ratio biases have been 
observed in countless other species (Karlin 1986). Explanations for biased sex ratios often focus on demographic differences (e.g., costs of offspring, mortality of specific life cycle stages); however, much sex ratio theory is based on purely verbal arguments or models with minimal demographic structure.

Early explanations of sex ratio evolution, for instance, relied on occasionally confused or vague verbal reasoning. Darwin (1871) wrote that parents producing more of the rarer sex would have fewer superfluous offspring and thus be more "productive," but later admitted the problem was too intricate for him to reason through (Darwin 1874). Fisher (1930) tackled this challenge with a famously succinct, and infamously cryptic, verbal argument based on reproductive value (the present value of an individual's future offspring). Because every individual has a male and female parent, Fisher stated that the "total reproductive value" of each sex in a given generation (i.e., their genetic contributions to all future generations, West 2009) must be equal.

If males and females are equally costly to produce, the sex ratio should evolve to equality, since any sex produced in excess will have fewer mating opportunities, less reproductive success, and thus smaller returns on reproductive value; parents who are genetically predisposed to producing the rarer sex thus have more grandchildren to propagate their genes, making the once rarer sex more common over time (Hamilton 1967, West 2009). If, however, males and females are differentially costly (e.g., require different amounts of resources to produce), Fisher claimed the sex ratio will evolve so that there is equal "expenditure" in, rather than equal numbers of, both sexes.

Trivers (1972) more precisely defined this expenditure as "parental investment" — any investment a parent makes (time, energy, resources, protection, etc.) to increase an offspring's survival and reproductive success, at the cost of investing in other children. If a son, for example, requires less parental investment than a daughter, a parent can produce more successful sons (and, to a point, more reproductive value) per unit investment. Selection thus biases the sex ratio towards sons until there is equal parental investment in sons and daughters. The optimal primary sex ratio $s_{1}^{*}$ is given by the "equal investment principle":

$$
\begin{aligned}
C_{m} s_{1}^{*} & =C_{f}\left(1-s_{1}^{*}\right) \\
s_{1}^{*} & =\frac{C_{f}}{C_{m}+C_{f}}
\end{aligned}
$$

where $C_{m}$ and $C_{f}$ are some form of male and female investment costs (Charnov 1982, Hardy 2002). Others (e.g., Charnov 1982, Bull and Charnov 1988, Frank 1990) have shown that the equal investment principle (3.1) requires several implicit assumptions, including random mating, fixed resource allocation, and additive offspring costs with linear returns (e.g., doubling your investment in sons doubles the grandchildren or genetic returns that your sons produce).

Early mathematical treatments of Darwin and Fisher's arguments by Düsing (1883, translated in Edwards 2000) and Shaw and Mohler (1953) are the basis for many other sex ratio analyses. They consider how an individual's sex ratio affects their fitness, through their relative number of 
(or genetic contribution to) grandchildren. The fitness $w$ of a given parent has the form:

$$
w=\frac{n}{4 N}\left(\frac{s_{1}}{S_{1}}+\frac{1-s_{1}}{1-S_{1}}\right)
$$

when that parent produces $n$ offspring at a primary sex ratio $s_{1}$, and the population at large produces $N$ offspring at a primary sex ratio $S_{1}$. This formulation does not consider stage structure within the sexes, nor does it account for offspring production over more than two generations.

The fitness of a given sex ratio phenotype $s_{1}$ is frequency-dependent, in that it depends on the population sex ratio $S_{1}$. When the population sex ratio $S_{1}=0.5,(3.2)$ is always $w=\frac{n}{2 N}$, regardless of the individual sex ratio $s_{1}$ (Shaw and Mohler 1953); this means that all sex ratios, including the resident and any mutants, will have the same fitness. Thus, when $S_{1}=0.5$, no individual sex ratio can have greater fitness than the resident, so no alternatives sex ratios can increase under selection. The equal sex ratio $S_{1}=0.5$ is thus an "unbeatable" evolutionarily stable strategy (ESS) (Maynard Smith and Price 1973). Subsequent studies have identified numerous factors that can bias the ESS sex ratio from 0.5, including local mate competition (Hamilton 1967), maternal condition (Trivers and Willard 1973), parent-offspring conflict (Trivers 1974, Trivers and Hare 1976), and other unusual life history strategies or sex determination systems (Hardy 2002).

The ESS sex ratios can be affected by sex-biased offspring costs, especially in terms of parental investment and the timing of sex-biased mortality (Hardy 2002, West 2009). Shaw and Mohler (1953) noted that sex-specific survival probabilities cancel out of (3.2) and are thus irrelevant to selection, though they did not consider parental investment. Fisher (1930) himself argued that only sex-biased mortality during the period of parental investment affects the sex ratio, and later analyses have largely ruled in favor of his conjecture. Similar to Shaw and Mohler, some frame sex ratio fitness in terms of a genetic contribution to grandchildren (Kolman 1960). Others use a population genetics approach to track the allele frequencies of different sex ratios (Leigh 1970). The general consensus is that sex-biased mortality after parental investment cannot bias the ESS sex ratio, because increased mortality is then compensated for by increased reproductive opportunities (West 2009).

However, few of these analyses explicitly consider stage structure, even though the effect of mortality at different life cycle stages is an inherently demographic issue. Many also only consider offspring production over two generations, rather than over an entire lifetime. Models that incorporate both age structure and lifetime offspring production are rarer and have produced more mixed results. In some age-structured models (Charnov 1975), sex-biased survival cancels out of the fitness expression, while in others (Emlen 1968a, b), mortality at all reproducing ages affects the sex ratio.

These discrepancies suggest that stage-specific, demographic factors deserve additional consideration in sex ratio theory. To this end, we use matrix population models to incorporate multiple sexes, stages, and life cycle events into our evolutionary projections. Furthermore, although 
previous studies of sex ratio evolution (e.g. Charnov 1979a, Charnov 1982, Hardy 2002, Otto and Day 2007) have focused on finding ESSs, additional methods are needed to determine whether the population will actually converge to the ESS in the long run. Here, we apply adaptive dynamics to identify potential evolutionary outcomes and characterize both their evolutionary and convergence stability.

\subsection{Model and methods}

There are two main approaches for studying sex ratio evolution (West 2009). One approach uses population or quantitative genetics to track the dynamics of allele frequencies (e.g. Eshel 1975, Uyenoyama 1979, Charlesworth 1977, Karlin and Lessard 1986). The other approach, which includes ESS theory and adaptive dynamics, ignores the often complex underlying genetics and instead focuses on trait phenotypes (e.g.,Charnov 1982, Hardy 2002, Otto and Day 2007). We will use the latter approach by considering population-level effects of the sex ratio phenotype.

Following the two-sex modeling framework introduced in Shyu and Caswell (xxa), we construct a series of continuous-time rate matrices that incorporate multiple sexes, stages, and life cycle events. Because these models are frequency-dependent, their long-term population growth rates are given by the dominant eigenvalue of the projection matrix at the equilibrium stage distribution (Pollak 1986, Haedler 1988, Caswell and Weeks 1986, Ianelli et al. 2005). By applying adaptive dynamics theory, we use these models to identify and characterize long-term evolutionary outcomes for the primary sex ratio - namely, singular strategies including, but not limited, to ESSs.

\subsubsection{The two-sex matrix model}

Consider a population with five stages: juvenile males $m_{1}$ and juvenile females $f_{1}$, adult males $m_{2}$ and adult females $f_{2}$, and reproducing unions $u$ (mated couples with one male and one female each). Single adults interact to form unions, which then produce new juvenile offspring (Figure 3-1). A summary of the variables, parameters, and matrices in this model is provided in Table 3.1.

The population vector at time $t$ is:

$$
\mathbf{n}(t)=\left(\begin{array}{c}
m_{1} \\
m_{2} \\
f_{1} \\
f_{2} \\
u
\end{array}\right)
$$

The total unions (pairs) formed per time is given by the nonlinear harmonic mean mating function:

$$
M(\mathbf{n})=\frac{2 m_{2} f_{2}}{m_{2}+f_{2}}
$$




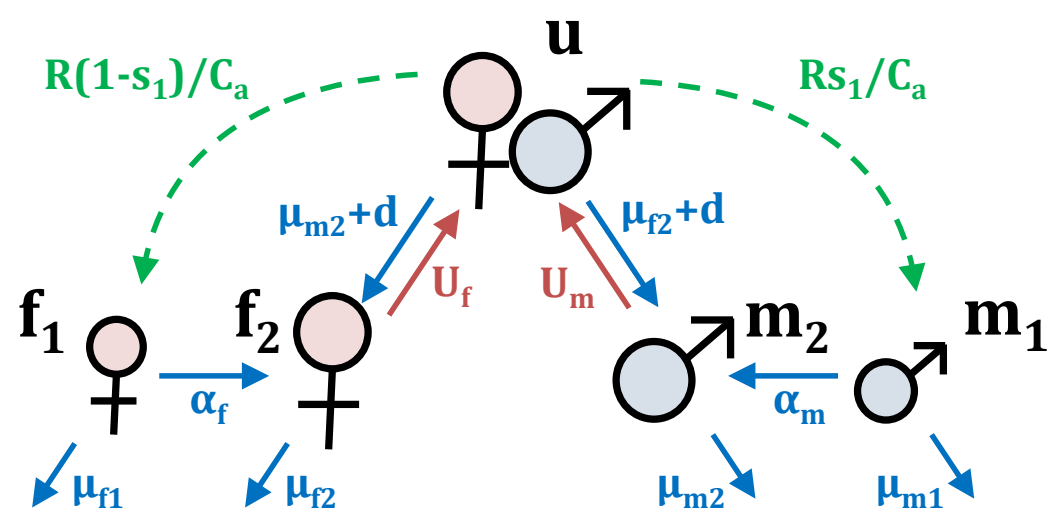

Figure 3-1: Life cycle diagram for a 5-stage population with juvenile males $m_{1}$ and juvenile females $f_{1}$, adult males $m_{2}$ and adult females $f_{2}$, and reproducing unions $u$. The functions and parameters shown here appear in the union formation matrix $\mathbf{U}$ (3.6) (red), birth matrix B (3.7) (green), or transition matrix $\mathbf{T}$ (3.8) (blue). (from Shyu and Caswell xxa)

which has frequency-dependent male and female per capita mating rates:

$$
\begin{aligned}
& U_{m}(\mathbf{n})=\frac{M(\mathbf{n})}{m_{2}}=\frac{2 f_{2}}{m_{2}+f_{2}} \\
& U_{f}(\mathbf{n})=\frac{M(\mathbf{n})}{f_{2}}=\frac{2 m_{2}}{m_{2}+f_{2}}
\end{aligned}
$$

Mating, birth, and life cycle transition processes are divided into three rate matrices $(\mathbf{U}, \mathbf{B}$, and T) as follows.

1. The union formation matrix $\mathbf{U}$ contains the per capita mating rates (3.5):

$$
\mathbf{U}(\mathbf{n})=\left(\begin{array}{ccccc}
0 & 0 & 0 & 0 & 0 \\
0 & -U_{m}(\mathbf{p}) & 0 & 0 & 0 \\
0 & 0 & 0 & 0 & 0 \\
0 & 0 & 0 & -U_{f}(\mathbf{p}) & 0 \\
0 & \frac{1}{2} U_{m}(\mathbf{p}) & 0 & \frac{1}{2} U_{f}(\mathbf{p}) & 0
\end{array}\right)
$$

2. The birth matrix $\mathbf{B}$ contains the rates of male and female offspring production by unions:

$$
\mathbf{B}=\left(\begin{array}{ccccc}
0 & 0 & 0 & 0 & \frac{R s_{1}}{C_{a}} \\
0 & 0 & 0 & 0 & 0 \\
0 & 0 & 0 & 0 & \frac{R\left(1-s_{1}\right)}{C_{a}} \\
0 & 0 & 0 & 0 & 0 \\
0 & 0 & 0 & 0 & 0
\end{array}\right)
$$


where $s_{1}$ is the (evolving) primary sex ratio, $R$ is the total resource investment rate, $C_{a}$ is the average offspring resource cost per birth. The quantity $R / C_{a}$ is the union reproductive rate (offspring produced per time).

3. The life cycle transition matrix $\mathbf{T}$ contains the rates of mortality and transitions between stages:

$$
\mathbf{T}=\left(\begin{array}{ccccc}
-\left(\mu_{m 1}+\alpha_{m}\right) & 0 & 0 & 0 & 0 \\
\alpha_{m} & -\mu_{m 2} & 0 & 0 & \mu_{f 2}+d \\
0 & 0 & -\left(\mu_{f 1}+\alpha_{f}\right) & 0 & 0 \\
0 & 0 & \alpha_{f} & -\mu_{f 2} & \mu_{m 2}+d \\
0 & 0 & 0 & 0 & -\left(\mu_{m 2}+\mu_{f 2}+d\right)
\end{array}\right)
$$

where $\mu_{m 1}$ and $\mu_{m 2}$ are the juvenile and adult male mortality rates, $\mu_{f 1}$ and $\mu_{f 2}$ are the juvenile and adult female mortality rates, $\alpha_{m}$ and $\alpha_{f}$ are the male and female maturation rates, and $d$ is the union divorce rate.

The average of these three rate matrices is the continuous-time projection matrix

$$
\mathbf{A}(\mathbf{n})=\frac{1}{3}[\mathbf{T}+\mathbf{B}+\mathbf{U}(\mathbf{n})]
$$

where

$$
\frac{d \mathbf{n}}{d t}=\mathbf{A}(\mathbf{n}) \mathbf{n}(t)
$$

In our model, the nonlinear mating rates (3.5) are homogeneous of degree 0 with respect to n. This allows all entries $a_{i j}$ in $\mathbf{A}$ to depend on relative stage frequencies rather than absolute abundances, i.e.:

$$
a_{i j}(c \mathbf{n})=a_{i j}(\mathbf{n})
$$

for any positive constant $c$. As a result, population growth is frequency-dependent, in that it is a function of the population frequency vector:

$$
\mathbf{p}=\frac{\mathbf{n}}{\|\mathbf{n}\|}
$$

where $\|\mathbf{n}\|$ is the 1 -norm of $\mathbf{n}$.

Frequency-dependent models like these ultimately converge to an equilibrium stage distribution $\hat{\mathbf{p}}$. The population then grows or decays exponentially at a rate given by the dominant eigenvalue $\lambda$ of $\mathbf{A}(\hat{\mathbf{p}})$. For calculating $\lambda$, it is sufficient to consider the dynamics of $\mathbf{p}$ (Shyu and Caswell xxa):

$$
\frac{d \mathbf{p}}{d t}=\left(\mathbf{I}_{s}-\mathbf{p} \mathbf{1}^{\top}\right) \mathbf{A}(\mathbf{p}) \mathbf{p}
$$


To find $\hat{\mathbf{p}}$, integrate (3.13) with the MATLAB ODE45 differential equation solver until $\mathbf{p}$ converges to $\hat{\mathbf{p}}$ (e.g., until vector entries do not change significantly over consecutive integration intervals). We then calculate the population's long-term growth rate $\lambda$, the dominant eigenvalue of $\mathbf{A}(\hat{\mathbf{p}})$, and its corresponding right and left eigenvectors $\mathbf{w}$ and $\mathbf{v}$. Note that the dominant right eigenvector of $\mathbf{A}(\hat{\mathbf{p}})$ equals the stable stage distribution; that is, $\mathbf{w}=\hat{\mathbf{p}}$.

\begin{tabular}{|c|c|}
\hline \multicolumn{2}{|c|}{ Matrices and Vectors } \\
\hline $\mathbf{A}$ & projection matrix $(3.9)$ \\
\hline B & birth matrix $(3.7)$ \\
\hline $\mathbf{U}$ & union formation matrix $(3.6)$ \\
\hline $\mathbf{T}$ & transition matrix $(3.8)$ \\
\hline $\mathbf{n}$ & population density vector (3.3) \\
\hline $\mathbf{p}$ & population frequency vector $(3.12)$ \\
\hline$\hat{\mathbf{p}}$ or $\mathbf{w}$ & equilibrium stage structure \\
\hline $\mathbf{v}$ & reproductive value vector \\
\hline \multicolumn{2}{|c|}{ Population Properties } \\
\hline$\lambda$ & long-term population growth rate, dominant eigenvalue of $\mathbf{A}(\hat{\mathbf{p}})$ \\
\hline$m_{1}, m_{2}$ & juvenile, adult male stages \\
\hline$f_{1}, f_{2}$ & juvenile, adult female stages \\
\hline$u$ & union (pair) stage \\
\hline$s_{1}$ & primary sex ratio (proportion of offspring that are born male) \\
\hline$s_{1}^{*}$ & singular strategy (SS) value of $s_{1}$ \\
\hline$s_{2}$ & secondary sex ratio (proportion of adults that are male) \\
\hline$s_{2}^{*}$ & resulting $s_{2}$ when $s_{1}=s_{1}^{*}$. \\
\hline$v_{i}$ & reproductive value of stage $i$ \\
\hline \multicolumn{2}{|c|}{ Life Cycle Parameters } \\
\hline$\alpha_{m}, \alpha_{f}$ & male, female maturation rates \\
\hline & divorce rate (rate at which a male-female pair bond breaks) \\
\hline$\mu_{f 1}, \mu_{f 2}$ & juvenile, adult female mortality rates \\
\hline$\mu_{m 1}, \mu_{m 2}$ & juvenile, adult male mortality rates \\
\hline$R$ & resource investment rate \\
\hline & mating function (3.4) (total unions formed per time) \\
\hline$U_{m}, U_{f}$ & per capita mating rates $(3.5)$ \\
\hline$F_{m}, F_{f}$ & per capita fertility rates $(3.63)$ \\
\hline \multicolumn{2}{|c|}{ Offspring Cost Parameters } \\
\hline$C_{m}, C_{f}$ & male, female offspring resource costs \\
\hline$C_{a}$ & average offspring resource cost $(3.35)$ \\
\hline$a$ & age of independence \\
\hline$I$ & offspring investment rate \\
\hline$D_{m}, D_{f}$ & male, female parental mortality costs \\
\hline$E_{m}, E_{f}$ & male, female parent costs of reproduction (3.42) \\
\hline$\mu_{m 2 c}, \mu_{f 2 c}$ & mated male, female mortality rates $(3.44)$ \\
\hline
\end{tabular}

Table 3.1: A summary of the variables, parameters, matrices, and population properties in the two-sex matrix model. Mutant parameters (not shown) are denoted by an apostrophe; e.g., $\mathbf{A}^{\prime}$ is the mutant projection matrix.

\subsubsection{Evolutionary analysis with adaptive dynamics}

Adaptive dynamics treats evolution as a series of "invasions" by mutant phenotypes. Mutations are assumed to occur infrequently, so that each mutation is either fixed or lost before the next mutation arises (Geritz et al. 1998). Because each mutant is initially rare, its effects on the existing resident population are considered negligible (Metz 2006). 
Consider a stable, monomorphic resident population with phenotype $x$, projection matrix $\mathbf{A}$, and growth rate $\lambda$. An invading mutant with phenotype $y$, projection matrix $\mathbf{A}^{\prime}$, and growth rate $\lambda^{\prime}$ (which depends on the environmental conditions set by the resident) has two possible fates. If $\lambda^{\prime}<\lambda$, the mutant will ultimately die out. But if $\lambda^{\prime}>\lambda$, the mutant can replace the resident and induce evolutionary change (Metz 1992).

\subsubsection{The mutant projection matrix}

Analogous to the resident projection matrix $\mathbf{A}$ in (3.9), the mutant projection matrix $\mathbf{A}^{\prime}$ is the average of the mutant rate matrices:

$$
\mathbf{A}^{\prime}(\hat{\mathbf{p}})=\frac{1}{3}\left[\mathbf{T}^{\prime}+\mathbf{B}^{\prime}+\mathbf{U}^{\prime}(\hat{\mathbf{p}})\right]
$$

The only phenotypic difference between mutants and residents is the primary sex ratio they use. Just as the resident birth matrix $\mathbf{B}$ in (3.7) depends on the resident sex ratio $s_{1}$, the mutant birth matrix $\mathbf{B}^{\prime}$ depends on mutant sex ratio $s_{1}^{\prime}$ :

$$
\mathbf{B}^{\prime}=\left(\begin{array}{ccccc}
0 & 0 & 0 & 0 & \frac{R s_{1}^{\prime}}{C_{a}} \\
0 & 0 & 0 & 0 & 0 \\
0 & 0 & 0 & 0 & \frac{R\left(1-s_{1}^{\prime}\right)}{C_{a}} \\
0 & 0 & 0 & 0 & 0 \\
0 & 0 & 0 & 0 & 0
\end{array}\right)
$$

Because mutants are so rare, we assume they mate only with residents. As a result, the equilibrium resident population sets the overall mating rate according to (3.5), and the mutant mating matrix $\mathbf{U}^{\prime}$ is the resident mating matrix $\mathbf{U}$ in (3.6) evaluated at the resident stable stage distribution $\hat{\mathbf{p}}$ :

$$
\mathbf{U}^{\prime}(\hat{\mathbf{p}})=\left(\begin{array}{ccccc}
0 & 0 & 0 & 0 & 0 \\
0 & -U_{m}(\hat{\mathbf{p}}) & 0 & 0 & 0 \\
0 & 0 & 0 & 0 & 0 \\
0 & 0 & 0 & -U_{f}(\hat{\mathbf{p}}) & 0 \\
0 & \frac{1}{2} U_{m}(\hat{\mathbf{p}}) & 0 & \frac{1}{2} U_{f}(\hat{\mathbf{p}}) & 0
\end{array}\right)
$$

Unless certain transition rates also depend on the primary sex ratio (e.g., parental survival in Section 3.4.5, which has transition matrix (3.43)), the mutant transition matrix $\mathbf{T}^{\prime}$ is the same as the resident transition matrix $\mathbf{T}$ in (3.8).

\subsubsection{Invasion fitness and the selection gradient}

We define the invasion fitness $s_{x}(y)$ as the long-term growth rate of a mutant with phenotype $y$, relative to the growth rate of a resident with phenotype $x$, in the equilibrium resident environment 
(as set by the resident's stable stage distribution $\hat{\mathbf{p}}$ ). In our two-sex matrix model, the invasion fitness is the difference in the dominant eigenvalues of the mutant and resident projection matrices ( $\lambda$ and $\lambda^{\prime}$, respectively), where $\lambda^{\prime}$ is evaluated at the resident's stable stage distribution $\hat{\mathbf{p}}$.

$$
s_{x}(y)=\lambda^{\prime}(\hat{\mathbf{p}})-\lambda
$$

Only mutants with a positive invasion fitness can displace the resident and cause evolutionary change.

The first derivative of the invasion fitness (3.17), with respect to the mutant phenotype $y$, is the selection gradient $D(x)$, which indicates the direction of selection at a given resident phenotype $x$. In our model, the selection gradient is the sensitivity of mutant eigenvalue $\lambda^{\prime}$ (Caswell 2010). In general, the resident and mutant phenotypes can be written as vectors of trait values, $\boldsymbol{\theta}$ and $\boldsymbol{\theta}^{\prime}$ respectively. The selection gradient is then:

$$
\begin{aligned}
D(x) & =\left.\frac{\partial s_{x}(y)}{\partial y}\right|_{y=x}=\left.\frac{\partial \lambda^{\prime}}{\partial \boldsymbol{\theta}^{\prime \top}}\right|_{\boldsymbol{\theta}^{\prime}=\boldsymbol{\theta}} \\
& =\left.\left(\left(\mathbf{w}^{\prime \top} \otimes \mathbf{v}^{\prime \top}\right) \frac{d \mathrm{vec} \mathbf{A}^{\prime}}{d \boldsymbol{\theta}^{\prime \top}}\right)\right|_{\boldsymbol{\theta}^{\prime}=\boldsymbol{\theta}}
\end{aligned}
$$

where $\mathbf{w}^{\prime}$ and $\mathbf{v}^{\prime}$ are the dominant right and left eigenvectors of the mutant matrix $\mathbf{A}^{\prime}(\hat{\mathbf{p}})$, scaled so that $\mathbf{v}^{\prime \top} \mathbf{w}^{\prime}=1$.

Here, we consider the case where the only evolving trait is the primary sex ratio. Thus, the trait vectors $\boldsymbol{\theta}$ and $\boldsymbol{\theta}^{\prime}$ simplify to the scalar resident and mutant sex ratios $s_{1}$ and $s_{1}^{\prime}$. The selection gradient at $s_{1}$ is thus:

$$
\left.\frac{\partial \lambda^{\prime}}{\partial s_{1}^{\prime}}\right|_{s_{1}^{\prime}=s_{1}}=\left.\left(\left(\mathbf{w}^{\prime \top} \otimes \mathbf{v}^{\prime \top}\right) \frac{d \operatorname{vec} \mathbf{A}^{\prime}}{d s_{1}^{\prime}}\right)\right|_{s_{1}^{\prime}=s_{1}}
$$

The selection gradient (3.19) can lend insight into both the transient and equilibrium evolutionary dynamics of $s_{1}$. Though we will focus on equilibrium results here, transient evolutionary dynamics can also be explored using the canonical equation (as discussed in Shyu and Caswell xxb).

\subsubsection{Singular strategies}

When the selection gradient (3.19) is 0 , there is no directional selection on $s_{1}$. The corresponding resident strategy $s_{1}^{*}$ is called a singular strategy (SS). MATLAB's f solve or fmincon functions can be used determine the values of $s_{1}$ where the selection gradient vanishes, which correspond to $s_{1}^{*}$.

Singular strategies are potential long-term evolutionary outcomes that can be characterized by several criteria (Geritz et al. 1998). One can, instance, determine whether each SS is evolutionarily stable (an ESS that is resistant to further invasion) or evolutionarily unstable (a branching point that leads to phenotypic divergence), as well as whether each SS is convergence stable (an evolutionary 
attractor that the population will converge to through small mutations) or convergence unstable (an evolutionary repeller).

For a one-dimensional phenotype, 2D visualizations of the invasion fitness landscape called pairwise invasion plots (PIPs) graphically indicate evolutionary and convergence stability. A PIP shows where invasion fitness is positive or negative, depending on the resident phenotype $x$ and the mutant phenotype $y$ (Figure 3-2).

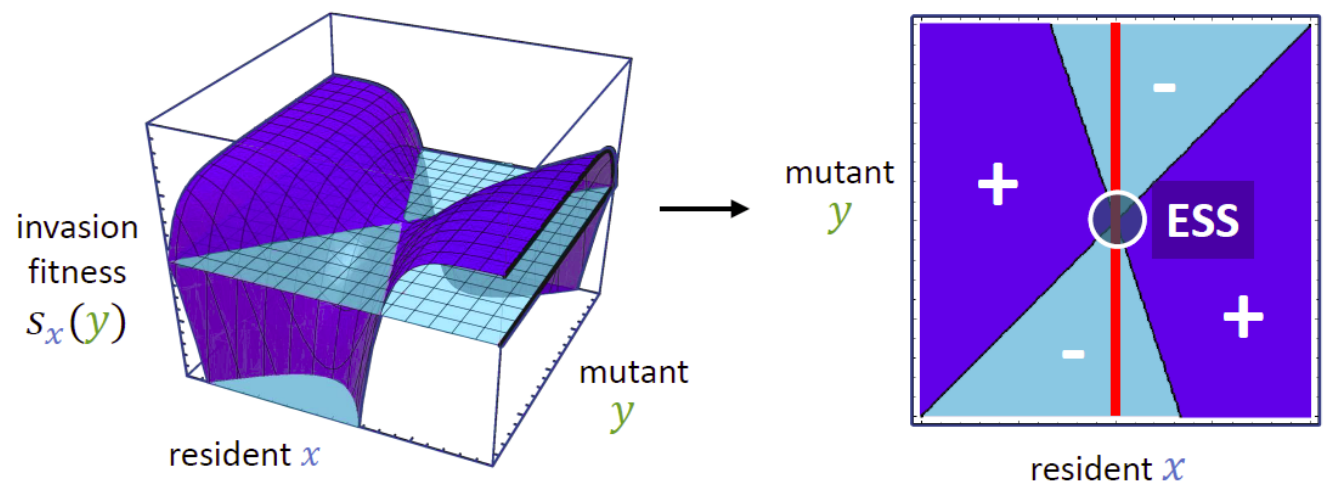

Figure 3-2: A 3D visualization of an invasion fitness landscape and the corresponding 2D pairwise invasion plot (PIP).

Singular strategies occur at intersections of the boundaries between negative and positive regions. If mutations are small (do not differ drastically from the resident phenotype), the behavior of the PIP around a SS indicates several properties (Figure 3-3). If, for example, the vertical line through the SS is entirely in the negative region (as in Figure 3-2), the SS is evolutionarily stable, i.e., an ESS resistant to further invasion.

\subsubsection{Second derivatives of invasion fitness}

Evolutionary and convergence stability can be determined more generally by using the local second derivatives of the invasion fitness $s_{x}(y)$ (3.17) to the mutant phenotype $y$ and the resident phenotype $x$ (Figure 3-3). In our two-sex model, these are the second derivatives of the mutant and resident eigenvalues, $\lambda^{\prime}$ and $\lambda$, with respect to the vectors describing mutant and resident phenotypes, $\boldsymbol{\theta}^{\prime}$ and $\boldsymbol{\theta}$. Again, we will only consider the primary sex ratio phenotype, so $\boldsymbol{\theta}$ and $\boldsymbol{\theta}^{\prime}$ simplify to $s_{1}$ and $s_{1}^{\prime}$ respectively.

The pure second derivative with respect to the mutant phenotype is:

$$
\frac{\partial^{2} s_{x}(y)}{\partial^{2} y}=\frac{\partial^{2}\left(\lambda^{\prime}-\lambda\right)}{\partial \boldsymbol{\theta}^{\prime} \partial \boldsymbol{\theta}^{\prime \top}}=\frac{\partial^{2} \lambda^{\prime}}{\partial^{2} s_{1}^{\prime}}
$$




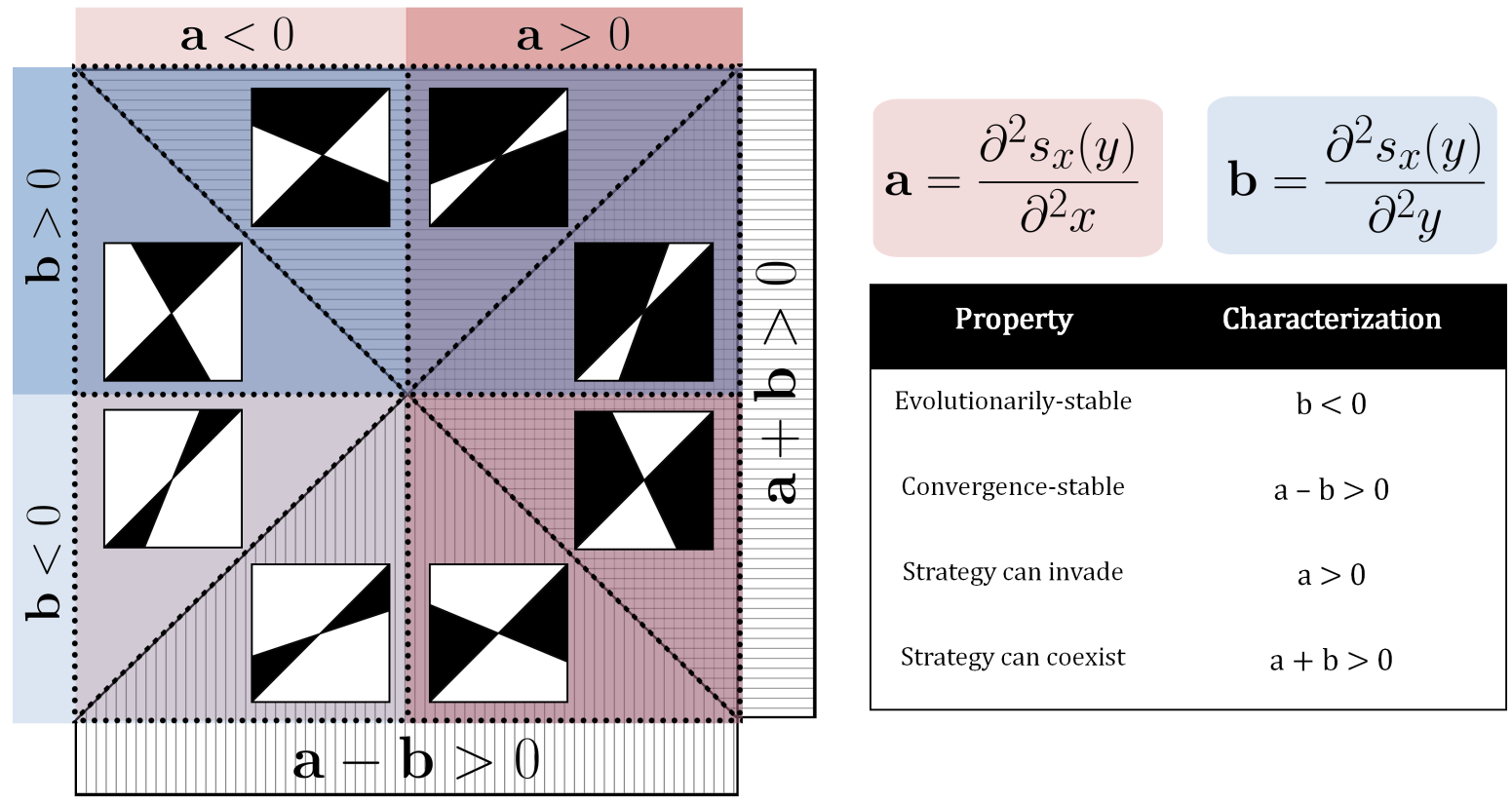

Figure 3-3: Second derivative properties and the corresponding pairwise invasion plots (PIPs) for the eight types of singular strategies (adapted from Geritz et al. 1998).

because $\lambda$ does not depend on the mutant sex ratio $s_{1}^{\prime}$.

The pure second derivative with respect to the resident phenotype is:

$$
\frac{\partial^{2} s_{x}(y)}{\partial^{2} x}=\frac{\partial^{2}\left(\lambda^{\prime}-\lambda\right)}{\partial \boldsymbol{\theta} \partial \boldsymbol{\theta}^{\top}}=\frac{\partial^{2}\left(\lambda^{\prime}-\lambda\right)}{\partial^{2} s_{1}}
$$

because both $\lambda^{\prime}$ and $\lambda$ depend on the resident sex ratio $s_{1}$.

The evolutionary stability of a singular strategy $x^{*}$ depends on (3.20) (Geritz et al. 1996):

$$
\left.\frac{\partial^{2} s_{x}(y)}{\partial^{2} y}\right|_{x=y=x^{*}} \begin{cases}<0 & \text { evolutionarily stable (ESS) } \\ =0 & \text { may be selectively neutral (weak form ESS) } \\ >0 & \text { evolutionarily unstable (branch point) }\end{cases}
$$

The convergence stability of a singular strategy $x^{*}$ depends on both (3.20) and (3.21) (Eshel 1983; Geritz et al. 1996):

$$
\left.\left(\frac{\partial^{2} s_{x}(y)}{\partial^{2} x}-\frac{\partial^{2} s_{x}(y)}{\partial^{2} y}\right)\right|_{x=y=x^{*}} \begin{cases}>0 & \text { convergence stable (attracting) } \\ <0 & \text { convergence unstable (repelling) }\end{cases}
$$


In next two sections, we will present matrix calculus equations for the pure second derivatives (3.20) and (3.21) that determine evolutionary and convergence stability. These expressions will rely on the equations (3.24) and (3.32) respectively.

\subsubsection{Second derivatives with respect to the mutant sex ratio (3.20)}

Calculating (3.20) requires the second derivatives of the mutant eigenvalue $\lambda^{\prime}$ with respect to the mutant trait $s_{1}^{\prime}$. The corresponding mutant matrix $\mathbf{A}^{\prime}$ is a function of the mutant trait $s_{1}^{\prime}$ and the resident's stable stage structure $\hat{\mathbf{p}}\left(s_{1}\right)$ (since mutants are rare, their environment is completely determined by the resident). Because $\hat{\mathbf{p}}$ is constant, $\mathbf{A}^{\prime}\left(s_{1}^{\prime}, \hat{\mathbf{p}}\right)$ is a constant matrix.

As shown in Shyu and Caswell $(2014,(38))$, the second derivatives of $\lambda^{\prime}$ can be found using matrix calculus:

$$
\frac{\partial^{2} \lambda^{\prime}}{\partial^{2} s_{1}^{\prime}}=\left(\mathbf{w}^{\prime \top} \otimes \mathbf{v}^{\prime \top} \otimes \mathbf{I}_{s}\right) H\left[\operatorname{vec} \mathbf{A}^{\prime} ; s_{1}^{\prime}\right]+\left(\frac{d \operatorname{vec} \mathbf{A}^{\prime}}{d s_{1}^{\prime}}\right)^{\top} H\left[\lambda^{\prime} ; \operatorname{vec} \mathbf{A}^{\prime}\right] \frac{d \operatorname{vec} \mathbf{A}^{\prime}}{d s_{1}^{\prime}}
$$

where $\otimes$ is the Kronecker product, vec is the vec operator, and $\mathbf{I}_{s}$ is a $s \times s$ identity matrix.

This expression depends on the Hessian (matrix of second derivatives) of $\lambda^{\prime}$ with respect to $\mathbf{A}^{\prime}$ :

$$
H\left[\lambda^{\prime} ; \operatorname{vec} \mathbf{A}^{\prime}\right]=\frac{1}{2}\left(\mathbf{H}_{1}+\mathbf{H}_{1}^{\top}\right)
$$

where

$$
\mathbf{H}_{1}=\left(\mathbf{I}_{n} \otimes \mathbf{v}^{\prime}\right) \frac{d \mathbf{w}^{\prime}}{d \operatorname{vec}^{\top} \mathbf{A}^{\prime}}+\left(\mathbf{w}^{\prime} \otimes \mathbf{I}_{n}\right) \frac{d \mathbf{v}^{\prime}}{d \operatorname{vec}^{\top} \mathbf{A}^{\prime}}
$$

and the first derivatives of $\mathbf{w}^{\prime}$ and $\mathbf{v}^{\prime}$ are

$$
\begin{aligned}
& \frac{d \mathbf{w}^{\prime}}{d \operatorname{vec}^{\top} \mathbf{A}^{\prime}}=\left(\lambda^{\prime} \mathbf{I}_{n}-\mathbf{A}^{\prime}+\mathbf{w}^{\prime} \mathbf{e}^{\top} \mathbf{A}^{\prime}\right)^{-1}\left[\mathbf{w}^{\prime} \boldsymbol{\otimes}\left(\mathbf{I}_{n}-\mathbf{w}^{\prime} \mathbf{e}^{\top}\right)\right] \\
& \frac{d \mathbf{v}^{\prime}}{d \operatorname{vec}^{\top} \mathbf{A}^{\prime}}=\left(\lambda^{\prime} \mathbf{I}_{n}-\mathbf{A}^{\prime \top}+\lambda^{\prime} \mathbf{v}^{\prime} \mathbf{w}^{\prime \top}\right)^{-1}\left(\left(\mathbf{I}_{n}-\mathbf{v}^{\prime} \mathbf{w}^{\prime \top}\right) \otimes \mathbf{v}^{\prime \top}-\lambda^{\prime}\left(\mathbf{v}^{\prime} \otimes \mathbf{v}^{\prime \top}\right) \frac{d \mathbf{w}^{\prime}}{d \operatorname{vec}^{\top} \mathbf{A}^{\prime}}\right) .
\end{aligned}
$$

where $\mathbf{e}^{\top}$ is a $1 \times s$ vector of ones.

The expression (3.24) also depends on the first and second derivatives of $\mathbf{A}^{\prime}$ with respect to $s_{1}^{\prime}$, which are given by $\frac{d \mathrm{vec} \mathbf{A}^{\prime}}{d s_{1}^{\prime}}$ and $H\left[\operatorname{vec} \mathbf{A}^{\prime} ; s_{1}^{\prime}\right]$ respectively. Recall from (3.9) that:

$$
\mathbf{A}^{\prime}=\frac{1}{3}\left(\mathbf{T}^{\prime}+\mathbf{B}^{\prime}+\mathbf{U}^{\prime}\right)
$$

The first derivatives of $\mathbf{A}^{\prime}$ to $s_{1}^{\prime}$ are:

$$
\frac{d \operatorname{vec} \mathbf{A}^{\prime}}{d s_{1}^{\prime}}=\frac{1}{3}\left(\frac{d \operatorname{vec} \mathbf{T}^{\prime}}{d s_{1}^{\prime}}+\frac{d \operatorname{vec} \mathbf{B}^{\prime}}{d s_{1}^{\prime}}+\frac{d \operatorname{vec} \mathbf{U}^{\prime}}{d s_{1}^{\prime}}\right)
$$


The second derivatives of $\mathbf{A}^{\prime}$ to $s_{1}^{\prime}$ are:

$$
H\left[\operatorname{vec} \mathbf{A}^{\prime} ; s_{1}^{\prime}\right]=\frac{1}{3}\left(H\left[\operatorname{vec} \mathbf{T}^{\prime} ; s_{1}^{\prime}\right]+H\left[\operatorname{vec} \mathbf{B}^{\prime} ; s_{1}^{\prime}\right]+H\left[\operatorname{vec} \mathbf{U}^{\prime} ; s_{1}^{\prime}\right]\right)
$$

These derivatives can be evaluated by hand or with a symbolic math program. Because not all of the matrices depend on $s_{1}^{\prime}\left(\mathbf{U}^{\prime}\right.$, for example, never does), both (3.29) and (3.30) may simplify considerably.

\subsubsection{Second derivatives with respect to the resident sex ratio (3.21)}

Calculating (3.21) requires the second derivatives of the mutant eigenvalue $\lambda^{\prime}$ and resident eigenvalue $\lambda$ with respect to the resident trait $s_{1}$. The resident matrix $\mathbf{A}$ is a function of the resident trait $s_{1}$ and the resident stable stage distribution $\hat{\mathbf{p}}\left(s_{1}\right)$ (since mutants are rare, they do not affect resident dynamics). Because the resident's dynamics depend on its own stage distribution, $\mathbf{A}(\hat{\mathbf{p}})$ is a nonlinear, frequency-dependent matrix.

Frequency dependence makes the second derivatives of $\lambda$ difficult to calculate directly. But once (3.20) is found using (3.24), (3.21) can be calculated using the relationship:

$$
\left.\left(\frac{\partial^{2} \lambda^{\prime}}{\partial^{2} s_{1}^{\prime}}+2 \frac{\partial^{2} \lambda^{\prime}}{\partial s_{1} \partial s_{1}^{\prime}}+\frac{\partial^{2}\left(\lambda^{\prime}-\lambda\right)}{\partial^{2} s_{1}}\right)\right|_{s_{1}^{\prime}=s_{1}=s_{1}^{*}}=0
$$

which holds at any singular strategy $s_{1}^{*}$ (Appendix 3.A).

The first term in (3.31) is given by (3.20). The second term in (3.31) is the mixed second derivatives of $\lambda^{\prime}$ to $s_{1}^{\prime}$ and $s_{1}$. This is shown in Appendix 3.B to be:

$$
\frac{\partial^{2} \lambda^{\prime}}{\partial s_{1} \partial s_{1}^{\prime}}=\left(\mathbf{w}^{\prime \top} \otimes \mathbf{v}^{\prime \top} \otimes \mathbf{I}_{s}\right) \mathbf{K}_{n^{2}, s} \frac{d \mathrm{vec} \mathbf{C}}{d \mathbf{w}^{\top}} \frac{d \mathbf{w}}{d s_{1}}+\mathbf{C}^{\boldsymbol{\top}}\left[\left(\mathbf{I}_{n} \otimes \mathbf{v}^{\prime}\right) \frac{d \mathbf{w}^{\prime}}{d s_{1}}+\left(\mathbf{w}^{\prime} \otimes \mathbf{I}_{n}\right) \frac{d \mathbf{v}^{\prime}}{d s_{1}}\right]
$$

where

$$
\mathbf{C}=\frac{d \mathrm{vec} \mathbf{A}^{\prime}}{d s_{1}^{\prime}}
$$

Thus, at any singular strategy, (3.21) can be found by substituting the pure second derivative (3.20) and mixed second derivative (3.32) into the relationship (3.31). The convergence stability condition (3.23) thus becomes:

$$
\frac{\partial^{2} \lambda^{\prime}}{\partial^{2} s_{1}^{\prime}}+\left.\frac{\partial^{2} \lambda^{\prime}}{\partial s_{1} \partial s_{1}^{\prime}}\right|_{x=y=x^{*}} \begin{cases}<0 & \text { convergence stable (attracting) } \\ >0 & \text { convergence unstable (repelling) }\end{cases}
$$

\subsection{Case studies: sex-biased offspring costs}

If the sexes are differentially costly, Fisher (1930) predicts that the sex ratio will evolve to favor the cheaper sex. However, there are many potential interpretations of offspring costs. One sex 
may be costlier because it requires more resources, has greater mortality, or more severely reduces parental survival or reproduction (Trivers 1985, Charnov 1982). Furthermore, these costs may occur at different points in an individual's lifetime (Figure 3-1).

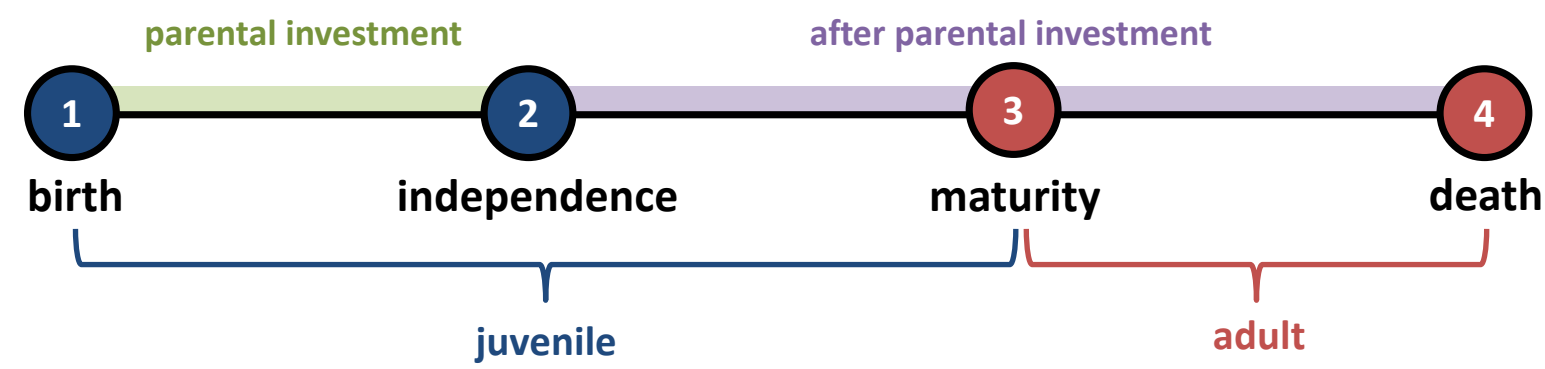

Figure 3-1: A timeline of key points in an individual's lifetime. The individual is a juvenile from Point 1 to 3 , and an adult from Point 3 to 4 . The period during parental investment is between Point 1 and 2; the period after parental investment is between Point 2 and 4 .

We consider four alternative interpretations of offspring costs (summarized in Table 3.1). For each of these four cases, we will determine how the primary sex ratio $s_{1}$ evolves with respect to singular strategy location, evolutionary stability, and convergence stability.

1. Offspring resource cost. Different amounts or rates of resources are required to birth male and female offspring (Figure 3-1, Point 1). Fisher's sex ratio theory implicitly assumes that parents have a fixed amount of resources for producing offspring (Bull and Charnov 1988). Here, we will assume that total resource investment is always constrained to a constant rate.

2. Offspring mortality cost (during investment). Male and female offspring mortality rates differ during the period of parental investment (Figure 3-1, Point 1 to 2); that is, while the offspring is still consuming parental resources. We assume that offspring death during this period frees up resources that can be reallocated to other offspring (Charnov 1982).

3. Offspring mortality cost (after investment). Male and female mortality rates differ after the period of parental investment, once the individual is no longer consuming parental resources. We will consider sex-biased mortality rates for both juveniles (Figure 3-1, Point 2 to 3 ) and adults (Figure $3-1$, Point 3 to 4 ).

4. Parent mortality cost. Male and female offspring differentially increase the mortality rates of their adult parents (Figure 3-1, Point 3 to 4).

In each case, we will consider a two-sex, 5-stage population with the life cycle in Figure 3-1. We construct projection matrices of the form (3.9), adapting the functions and parameters in rate matrices $\mathbf{U}$ (3.6), B (3.7), and $\mathbf{T}$ (3.8) as necessary to reflect the offspring costs under consideration. The corresponding resident and mutant matrices will be used to calculate the selection gradient (3.19), from which we can find the SS primary sex ratio $s_{1}^{*}$. We will determine the location and 


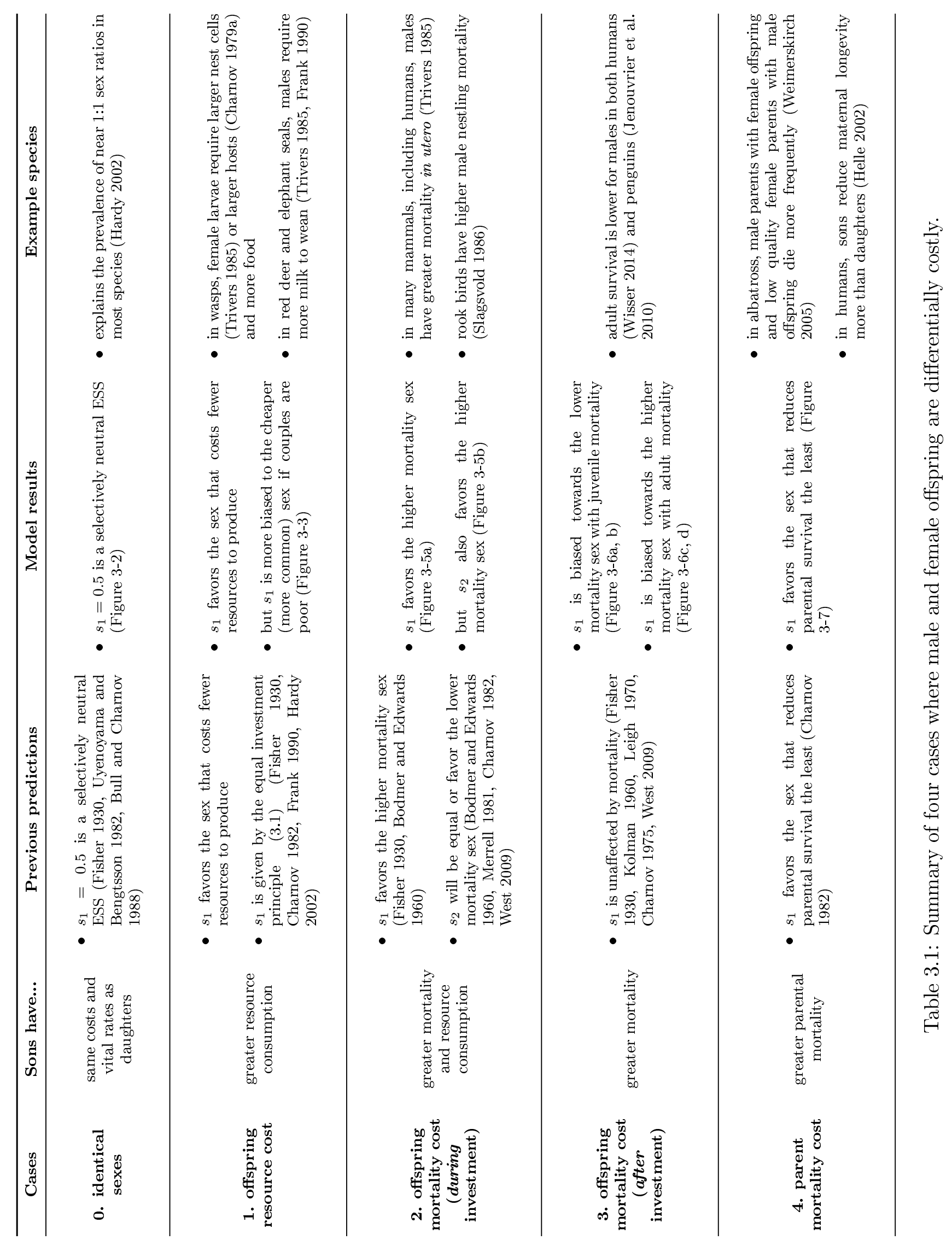


stability properties of $s_{1}^{*}$ in each case. We will also examine the secondary sex ratio $s_{2}$ (proportion of adults that are male), focusing specifically on $s_{2}^{*}$ as the value of $s_{2}$ when the primary sex ratio ratio $s_{1}$ is at its $\mathrm{SS}$ value $s_{1}^{*}$.

We make the following assumptions for the underlying two-sex model:

- Males and females have identical vital rates, save for the offspring cost of interest.

- Only the union stage can produce new offspring. Unmated males and females mate to form unions, but do not reproduce independently.

- Males are always the more "disadvantaged" sex, which is often true in mammals and birds (Table 3.1). In Case 1, males have higher resource costs. In Case 2 and 3, males have higher mortality. In Case 4, males impose greater parental mortality. An increase in $s_{1}$ thus represents greater production of the disadvantaged sex, while a decrease in $s_{1}$ represents increased production of the advantaged sex.

We also make these assumptions for our evolutionary analyses:

- The only evolving trait is the primary sex ratio $s_{1}$. Thus, new mutants only differ from established residents in terms of their sex ratio phenotype.

- Mutations are small and do not differ drastically from the resident. They are also rare enough not to affect the resident population, and infrequent enough to either die out or reach fixation before the next mutation arises (Geritz 1996, Metz 2006).

- The mutant phenotype is genetically dominant. Any offspring with a mutant parent also has the mutant phenotype.

Unless otherwise indicated, model parameters are fixed at the values in Table 3.2. We will consider example parameter sets for two types of unions, "productive" and "poor," in particular. Productive unions are more persistent (low divorce rate $d$ ) and can allocate more resources to offspring production (high resource investment rate $R$ ). Poor unions, in contrast, are more transient (high $d$ ) and allocate fewer resources to offspring production (low $R$ ).

\begin{tabular}{llcc}
\hline Parameter & Description & Value (Productive) & Value (Poor) \\
\hline$\mu_{m 1}, \mu_{f 1}$ & male, female juvenile mortality rates & 0.1 & 0.1 \\
$\mu_{m 2}, \mu_{f 2}$ & male, female adult mortality rates & 0.1 & 0.1 \\
$\alpha_{m}, \alpha_{f}$ & male, female maturation rates & 0.5 & 0.5 \\
$R$ & total resource investment rate & 20 & 10 \\
$d$ & union divorce rate & 0 & 1 \\
\hline
\end{tabular}

Table 3.2: Two-sex matrix model parameters for productive and poor unions. 


\subsubsection{Case 0: identically costly sexes}

If the sexes are identically costly, selection should favor equal production of males and females. The equal sex ratio is thus an "unbeatable" ESS resistant to invasion by alternative sex ratios (Hamilton 1967, Maynard Smith and Price 1973). Consistent with this classic prediction, our model has a convergent singular strategy at $s_{1}^{*}=0.5$ (example in Figure 3-2). This result is robust to other variants of our model, including the various cases of offspring costs, additional stages, different mating functions, etc.

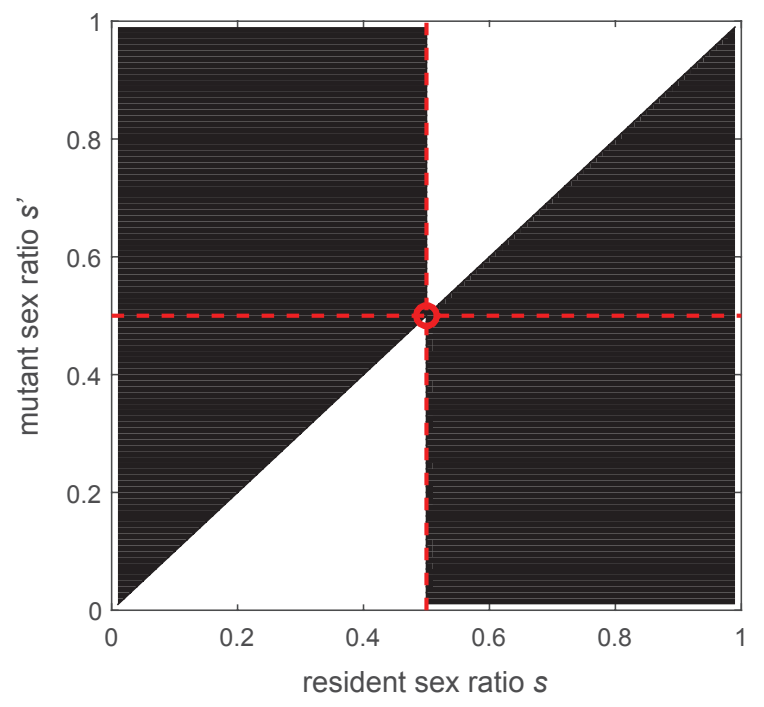

Figure 3-2: A PIP for sex ratio evolution when males and females are identical. With the parameters in Table 3.2 (Case 1, productive unions), (3.22) is 0 and (3.34) is -0.92 at $s_{1}^{*}=0.5$, confirming that the equal sex ratio is a selectively neutral, convergence stable ESS.

In all cases, the singular strategy intersects with a vertical isocline where mutant and resident growth rates are equal. This is because any mutant that arises when the resident is at the equal sex ratio has an invasion fitness $(3.17)$ of 0 . When $s_{1}^{*}=0.5$, resident males and females are equally abundant at equilibrium. Rare mutants thus have equal mating opportunities with residents regardless of their sex, so all invading sex ratios equally fit. As a result, the equal sex ratio is called a "selectively neutral" strategy (Bull and Charnov 1988) or a "weak form ESS" (Uyenoyama and Bengtsson 1982).

As we shall see in Section 3.4.6, convergence stable, selectively neutral sex ratios like these are the predominant singular strategies in our model.

\subsubsection{Case 1: offspring resource costs}

Consider the case where the production of male and female offspring requires different amounts of resources. These production costs are upfront, immediate investments made per birth or conception, and are thus unaffected by later offspring mortality. Parents have a fixed total rate $R$ at which they 
invest resources (energy, food, etc.) into offspring production, so the primary sex ratio $s_{1}$ determines how resources are allocated between the sexes.

Producing male offspring requires $C_{m}$ units of resources per time, while producing female offspring requires $C_{f}$ units of resources per time. The average resource cost per offspring birth is thus:

$$
C_{a}=s_{1} C_{m}+\left(1-s_{1}\right) C_{f}
$$

This average offspring cost appears in the birth matrix B, as given by (3.7), and determines how many offspring can be born per time. The union formation matrix $\mathbf{U}$ and transition matrix $\mathbf{T}$ are given by (3.6) and (3.8).

If demographic structure is ignored, the equal investment principle implies that the primary sex ratio will evolve to favor the cheaper sex. Assume, for example, that $C_{m}+C_{f}=1$; that is, there is some sort of offspring production tradeoff, so that as males become less costly, females become more costly, and vice versa. Then by (3.1):

$$
s_{1}^{*}=\frac{C_{f}}{C_{m}+C_{f}}=C_{f}
$$

The SS sex ratio $s_{1}^{*}$ increases, becoming more male-biased, as the female cost $C_{f}$ increases. Similarly, $s_{1}^{*}$ decreases, becoming more female-biased, as the male cost $C_{m}=1-C_{f}$ increases.

a)

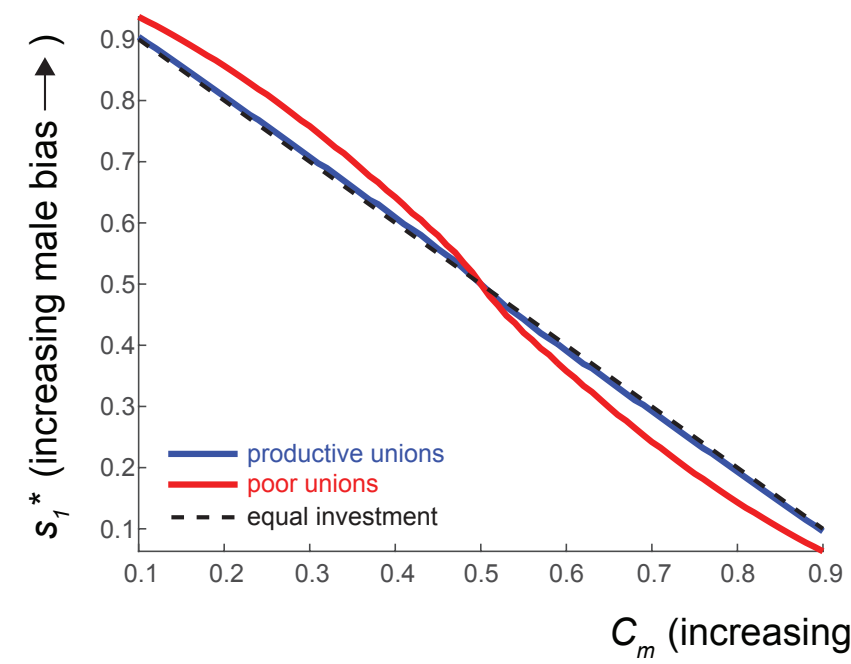

b)

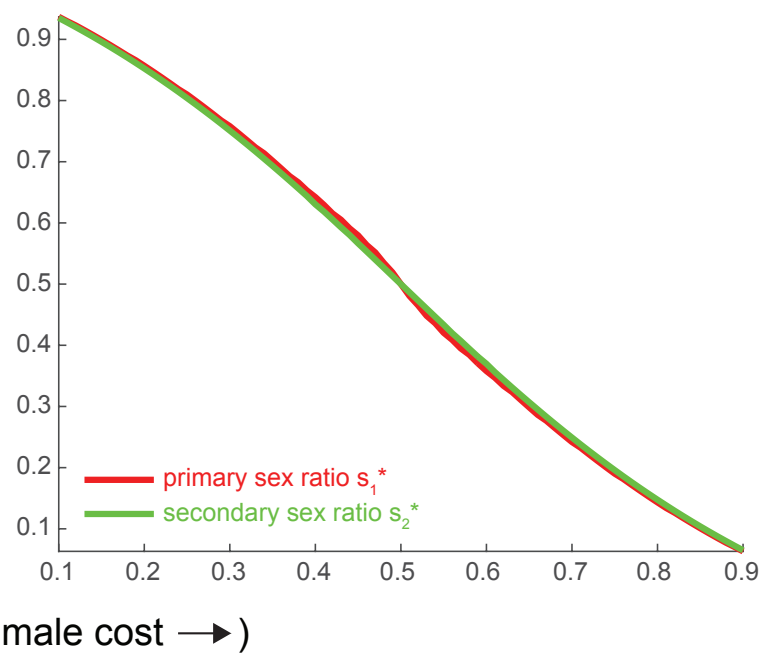

Figure 3-3: Case 1 singular strategy (SS) sex ratios, as a function of the male offspring cost $C_{m}$ in (3.35). In this example, the female offspring cost $C_{f}=1-C_{m}$. a) The primary sex ratio $s_{1}^{*}$ for both productive (blue) and poor unions (red). The values of $s_{1}^{*}$ predicted by the equal investment principle (3.36) are indicated in black. b) The corresponding secondary sex ratio $s_{2}^{*}$ (green) in the poor unions case.

Consistent with the predictions of the equal investment principle (3.36), the evolutionarily singular sex ratios in our demographic model are biased towards the cheaper sex (Figure 3-3a). 
For poor unions, however, $s_{1}^{*}$ deviates from the predictions of the equal investment principle in even greater favor of the cheaper sex. This is because the optimal sex ratio depends on a tradeoff between the cost of offspring production (where the cheaper sex is favored because more of it can be produced) and the benefit of offspring reproductive success (where the rarer sex is favored because it has more mating opportunities). However, an increase in mating opportunities is not necessarily proportional to an increase in later births, especially when unions have low reproductive output. When unions are poor, the mating advantage of the rarer, costlier sex is diminished, allowing the tradeoff to skew in favor of the more common, cheaper sex.

Recall that $s_{2}^{*}$ is the secondary sex ratios when $s_{1}=s_{1}^{*}$. In this case, males and females have the same maturation and mortality rates; thus, both $s_{2}^{*}$ and $s_{1}^{*}$ have the same values (Figure $3-3 \mathrm{~b}$ ).

\subsubsection{Case 2: offspring mortality during parental investment}

Rather than paying a single upfront production cost per birth, like in Case 1, parents now pour investments into their offspring over an extended period of time (the period of parental investment shown in Figure 3-1, Points 1 to 2). An offspring's cost (how much parental investment they have consumed) thus accumulates over time, and the cumulative cost of each offspring depends on how long they receive parental investment.

An offspring stops receiving parental investment only when it has reached the age of independence or died. As a result, the expected cost per offspring born depends on the juvenile mortality rates. If more male offspring die before reaching independence, for example, the average cost per male born will be less than that of a female. The average cost per male reared to independence, however, will be higher than that of a female (Fisher 1930).

Assume that males and females have different juvenile mortality rates, and will thus have different expected costs per birth. Again, parents invest in offspring at a fixed resource rate $R$. If male offspring have higher mortality rates, the average male born will consume fewer parental resources (and have a lower expected cost) than the average female born.

First, we will determine the expected offspring costs, per male or female born, as a function of the male and female juvenile mortality rates. As in Slagsvold (1986), let $I(x)$ be the instantaneous parental investment rate in an offspring at age $x$. A parent's cumulative investment $J(x)$ in that offspring up to age $x$ is:

$$
J(x)=\int_{0}^{x} I(z) d z
$$

Let $a$ be the age of independence, after which parental investment ceases. If the investment rate is constant so that $I(x)=I,(3.37)$ becomes:

$$
J(x)= \begin{cases}I x, & \text { if } x<a \\ I a, & \text { if } x \geq a\end{cases}
$$


Define $f(x)$ as the probability that an offspring dies at age $x$. If $\mu(x)$ is the mortality rate at age $x$, it can be shown that (Caswell 2001, Chapter 2):

$$
\begin{aligned}
f(x) & =\mu(x) e^{-\int_{0}^{x} \mu(z) d z} \\
& =\mu e^{-\mu x} \quad \text { if } \mu \text { is constant for all } x
\end{aligned}
$$

The expected cumulative investment in an offspring, accounting for its mortality rate during the investment period, is:

$$
\begin{aligned}
E[J(x)] & =\int_{0}^{\infty} J(x) f(x) d x \\
& =\int_{0}^{\infty} J(x) \mu e^{-\mu x} d x \\
& =\int_{0}^{a} J(x) \mu e^{-\mu x} d x+\int_{a}^{\infty} J(x) \mu e^{-\mu x} d x \\
& =\int_{0}^{a} I x \mu e^{-\mu x} d x+\int_{a}^{\infty} I a \mu e^{-\mu x} d x \\
& =\frac{I}{\mu}\left(1-e^{-\mu a}\right)
\end{aligned}
$$

Equation (3.40) is the expected cost per offspring birth with an offspring mortality rate of $\mu$. Male offspring will have a mortality rate $\mu_{m 1}$, while female offspring have a mortality rate $\mu_{f 1}$. If males and females receive parental investment at the same constant rate $I$, and have ages of independence $a_{m}=1 / \alpha_{m}$ and $a_{f}=1 / \alpha_{f}$ respectively, the expected male and female offspring costs per birth are thus:

$$
\begin{aligned}
C_{m} & =\frac{I}{\mu_{m 1}}\left(1-e^{-\frac{\mu_{m 1}}{\alpha_{m}}}\right) \\
C_{f} & =\frac{I}{\mu_{f 1}}\left(1-e^{-\frac{\mu_{f 1}}{\alpha_{f}}}\right)
\end{aligned}
$$

Substitute (3.41) into (3.35) to obtain $C_{a}$, the average offspring cost per birth. Again, the union formation matrix $\mathbf{U}$, birth matrix $\mathbf{B}$, and transition matrix $\mathbf{T}$ are given by (3.6), (3.7), (3.8) respectively.

Figure 3-4 shows that the resource cost for a given sex declines as its mortality rate increases, since more and more offspring die before significant parental investment is made. Consider, for example, the case where sons experience greater juvenile mortality than daughters. The average resource cost (3.41) of each son born is less than that of a daughter, because sons are more likely to die before consuming the full amount of resources needed to reach independence (Bodmer and Edwards 1960, West 2009, Kahn et al 2015). Based on the equal investment principle (3.1), we would expect the primary sex ratio to evolve in favor of the higher mortality (lower cost) sex. 


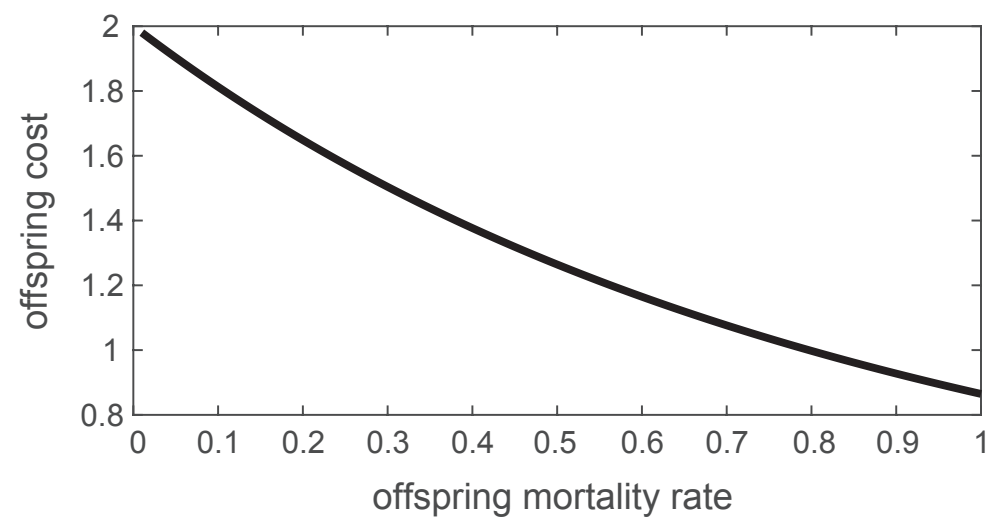

Figure 3-4: The offspring resource cost (3.41) as a function of the juvenile mortality rate $\mu$ ( $I=1$, $\alpha=0.5)$.

a)

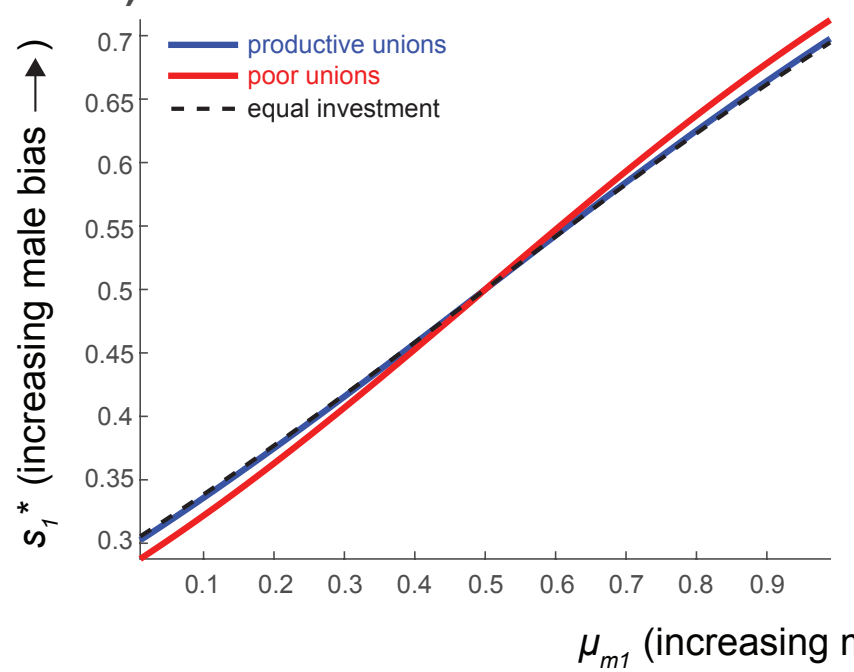

b)

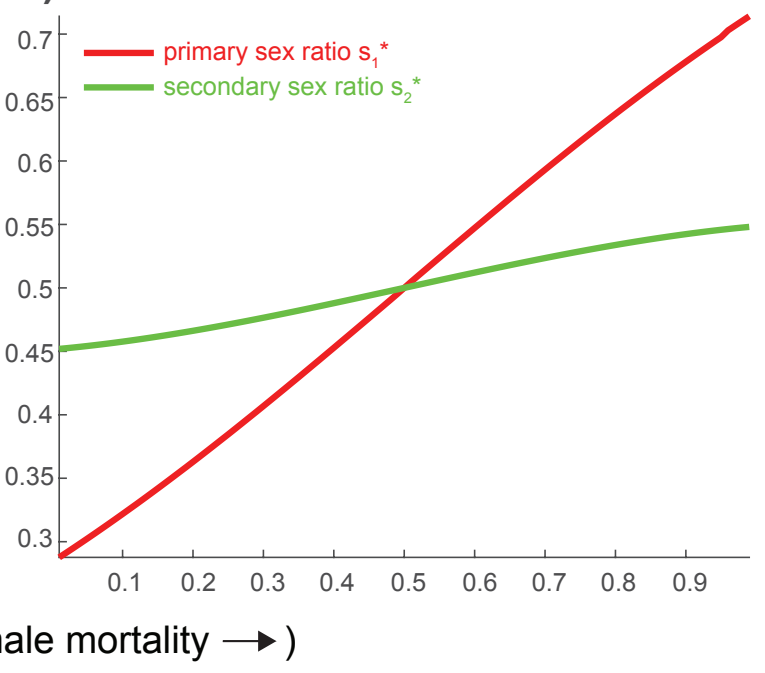

Figure 3-5: Case 2 singular strategy (SS) sex ratios, as a function of the juvenile male mortality rate $\mu_{m 1}$ in (3.41). We will set the juvenile female mortality rate $\mu_{f 1}=1-\mu_{m 1}$, so that increasing male mortality decreases female mortality and vice versa. a) The primary sex ratio $s_{1}^{*}$ for both productive (blue) and poor unions (red). The values of $s_{1}^{*}$ predicted by the equal investment principle (3.1) are indicated in black. b) The corresponding secondary sex ratio $s_{2}^{*}$ (green) in the poor unions case. 
In our model, $s_{1}^{*}$ is indeed biased towards the higher mortality (lower cost) males. As in Case 1, deviations from the equal investment principle increase when unions are poor (Figure 3-5a) again, because the mating advantage of the rarer, costlier sex is seemingly insufficient to compensate for its greater cost. The corresponding secondary sex ratio $s_{2}^{*}$ is less biased than the primary sex ratio, because the cheaper, higher mortality males produced in excess at birth are more likely to die before reaching maturity (Figure 3-5b). These results are consistent with the predictions of Fisher (1930), which state that, when the average expenditure is less for each boy born, boys will be more numerous at birth, but less numerous by the end of parental expenditure.

Although $s_{2}^{*}$ is less biased than $s_{1}^{*}$, however, both sex ratios are still biased towards the higher mortality sex. This contradicts previous arguments that the sex ratio should equalize to 0.5 by the age of independence (Bodmer and Edwards 1960) or reproduction (Merrell 1981), or that the sex ratio should favor the lower mortality daughters by the end of parental investment (Charnov 1982). Though the higher mortality sex does become less numerous by maturity (as Fisher originally stated), whether the sex ratio bias equalizes or even reverses is not absolute and likely depends on other factors (e.g., Kahn 2015).

\subsubsection{Case 3: offspring mortality after parental investment}

Suppose that male and female mortality rates differ after the period of parental investment (Figure 3-1, Points 2 to 4). Because sex-biased risks for disease, competition, selective harvest pressure, etc. can act at any point in the life cycle, we will consider both sex-biased juvenile mortality $\left(\mu_{m 1} \neq \mu_{f 1}\right)$ and sex-biased adult mortality $\left(\mu_{m 2} \neq \mu_{f 2}\right)$.

In Case 3, male and female offspring have the same resource costs $C_{m}=C_{f}$, which we shall normalize to 1 . Then the average offspring cost $C_{a}$ in (3.35) is also always 1 , and the union reproductive rate $R / C_{a}$ depends only on the (constant) resource investment rate $R$. Since offspring mortality does not affect the offspring resource costs, all mortality must occur after the period of investment. Thus, any juvenile mortality in Case 3 occurs in the period between independence and sexual maturity (Figure 3-1, Points 2 to 3 ).

Again, the union formation matrix $\mathbf{U}$, birth matrix $\mathbf{B}$, and transition matrix $\mathbf{T}$ given by (3.6), (3.7), and (3.8) respectively. We will fix the stage-specific mortality rates in $\mathbf{T}$ at different levels and analyze the sex ratios that evolve.

\subsubsection{Juvenile mortality}

Consider the case of sex-biased juvenile mortality after parental investment. When unions are productive (Figure 3-6a, blue), $s_{1}^{*}$ varies slightly as a function of juvenile mortality. This contradicts the predictions of Fisher and many others, who maintain that sex-biased mortality after parental investment does not affect sex ratio.

However, when unions are poor (Figure 3-6a, red), $s_{1}^{*}$ favors the lower mortality sex. This bias occurs for reasons similar to those in Cases 1 and 2. When unions are less productive, the increased 
a)

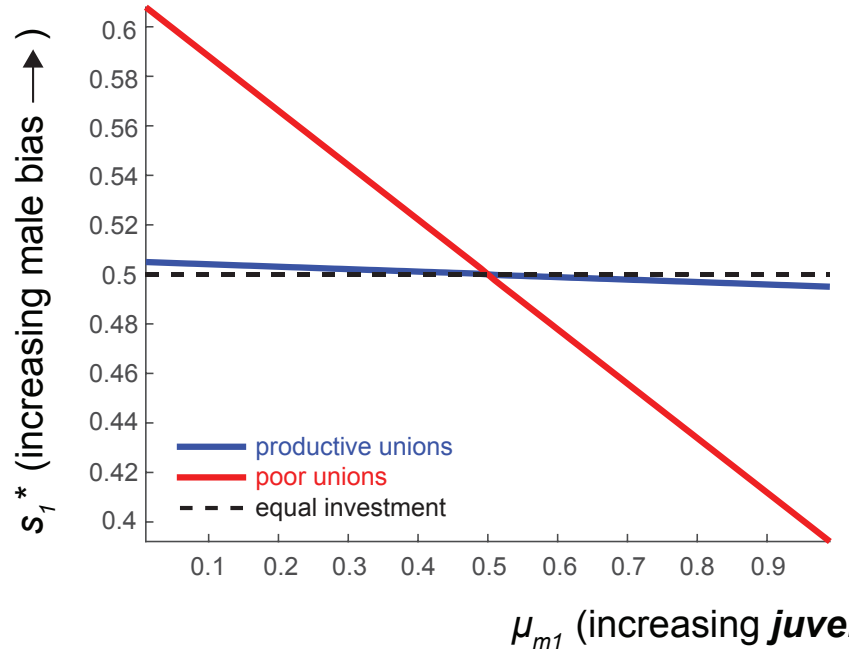

b)

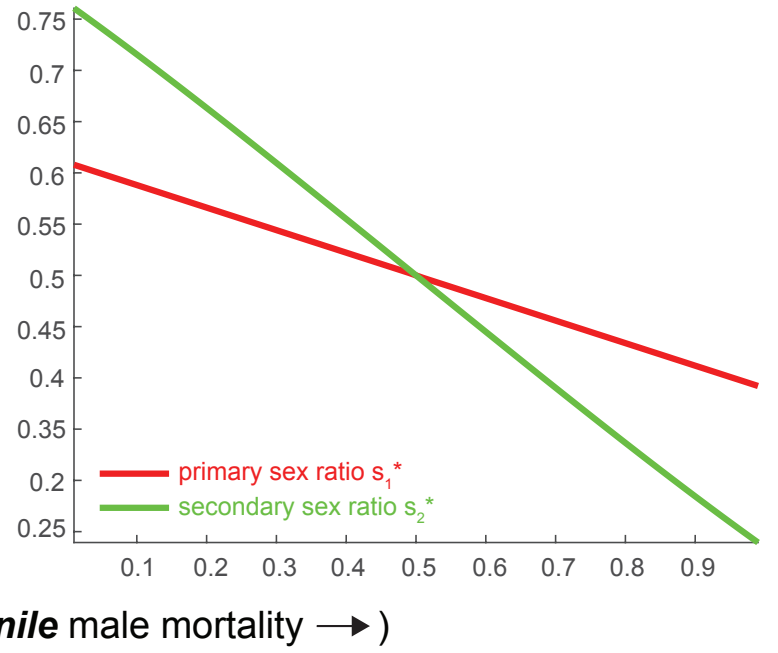

c)

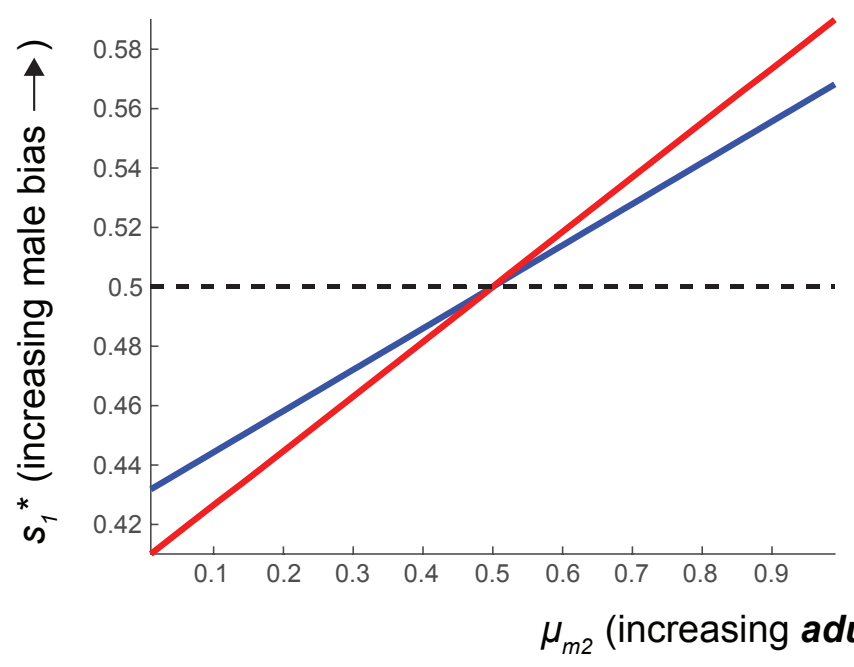

d)

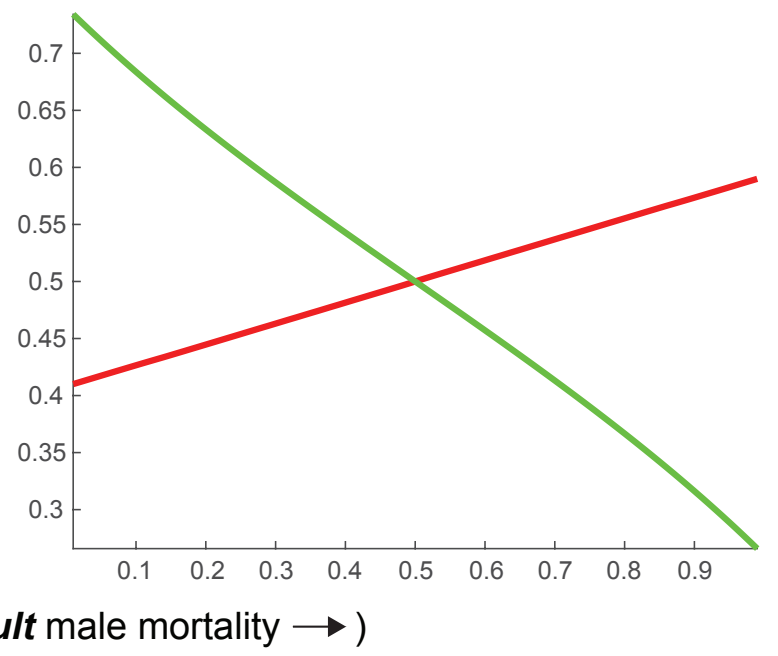

Figure 3-6: Case 3 singular strategy (SS) sex ratios, as a function of the juvenile male mortality rate $\mu_{m 1}$ or adult male mortality rate $\mu_{m 2}$ in (3.8). In the juvenile mortality case, the juvenile female mortality rate $\mu_{f 1}=1-\mu_{m 1}$. In the adult mortality case, the adult female mortality rate $\mu_{f 2}=1-\mu_{m 2}$. a) The primary sex ratio $s_{1}^{*}$ for both productive (blue) and poor unions (red) in the juvenile mortality case. The values of $s_{1}^{*}$ predicted by the equal investment principle (3.36) are indicated in black. b) The corresponding secondary sex ratio $s_{2}^{*}$ (green) in the poor unions, juvenile mortality case. c) Primary sex ratios for the adult mortality case. d) Secondary sex ratios for the poor unions, adult mortality case. 
mortality of a given sex is not compensated for by its increased mating rates, causing the sex ratio to favor the lower mortality sex.

The secondary sex ratio $s_{2}^{*}$ is even more biased towards the lower mortality sex than the primary sex ratio (Figure 3-6b). This is the opposite of Case 2 (mortality occurs during parental investment), where the secondary sex ratio was less biased, but both sex ratios still favored the higher mortality sex. This difference can be explained as follows.

When mortality occurs during parental investment (Case 2), the primary sex ratio favors the higher mortality sex. But although more of the higher mortality sex is produced at birth, that sex is also more likely to die before reaching maturity. As a result, both the primary and secondary sex ratios may favor the higher mortality sex, but the secondary sex ratio somewhat less so. When mortality occurs after parental investment (Case 3, juvenile mortality), the primary sex ratio favors the lower mortality sex. Not only is the lower mortality sex more likely to be produced at birth, but it also has less mortality later on. Thus, both the primary and secondary sex ratios favor the lower mortality sex, the secondary sex ratio somewhat more so.

\subsubsection{Adult mortality}

Unlike juvenile mortality, adult mortality expedites union dissolution through the death of mating partners. The return of widows and widowers to the available singles pool subsequently increases mating opportunities for the rarer sex. As a result, $s_{1}^{*}$ actually favors the rarer, higher mortality sex (Figure 3-6c), the opposite of the bias in the juvenile mortality case (Figure 3-6a).

Once again, these results contradict the Fisherian notion that mortality after parental investment cannot bias the primary sex ratio. As in the case of juvenile mortality, the magnitude of the sex ratio bias is modulated by union productivity. Productive unions have less sex ratio bias, possibly because their larger resource investment rate $R$ compensates for unions dissolving due to adult mortality. However, increasing the divorce rate $d$ may also reduce sex ratio bias, as the mating advantage of the higher mortality sex is reduced when unions dissolve more easily.

Although the primary sex ratio now favors the higher mortality sex, adult mortality is high enough to skew the secondary sex ratio $s_{2}^{*}$ towards the lower mortality sex (Figure 3-6d).

\subsubsection{Case 4: parental mortality}

Consider the case where male and female offspring impose different costs on the survival of their parents. As in Case 3, we will assume equal male and female offspring resource costs, so that $C_{m}=C_{f}=C_{a}=1$. In Case 4 , however, males and females have the same mortality rates, and differ instead in the extra mortality costs, $D_{m}$ and $D_{f}$, that they impose on their parents.

We assume there is a tradeoff between reproduction and survival, so that parents with a greater total cost of reproduction have greater mortality rates. Reproduction costs depend on the per capita mating function $U_{m}$ from (3.5), the resource investment rate $R$, the primary sex ratio $s_{1}$, and the male and female parental mortality costs $D_{m}$ and $D_{f}$. 
Let $E_{m}$ and $E_{f}$ be the expected cost of reproduction per male parent and per female parent respectively. Then:

$$
\begin{aligned}
E_{m} & =U_{m} R\left[s_{1} D_{m}+\left(1-s_{1}\right) D_{f}\right] \\
E_{f} & =U_{f} R\left[s_{1} D_{m}+\left(1-s_{1}\right) D_{f}\right]
\end{aligned}
$$

Note the parental mortality cost imposed per offspring is fixed at birth as $D_{m}$ or $D_{f}$, regardless of later offspring mortality. Alternatively, parents could continue incurring mortality costs until their offspring have either died or fully matured.

Because only adults in the union stage $u$ produce offspring, adults in the single unmated stages $m_{2}$ and $f_{2}$ do not experience this extra offspring-induced mortality. The transition matrix $\mathbf{T}$ (3.8) must now distinguish between unmated adult mortality rates $\left(\mu_{m 2}\right.$ and $\left.\mu_{f 2}\right)$ and mated adult mortality rates $\left(\mu_{m 2 c}\right.$ and $\left.\mu_{f 2 c}\right)$.

$$
\mathbf{T}=\left(\begin{array}{ccccc}
-\left(\mu_{m 1}+\alpha_{m}\right) & 0 & 0 & 0 & 0 \\
\alpha_{m} & -\mu_{m 2} & 0 & 0 & \mu_{f 2 c}+d \\
0 & 0 & -\left(\mu_{f 1}+\alpha_{f}\right) & 0 & 0 \\
0 & 0 & \alpha_{f} & -\mu_{f 2} & \mu_{m 2 c}+d \\
0 & 0 & 0 & 0 & -\left(\mu_{m 2 c}+\mu_{f 2 c}+d\right)
\end{array}\right)
$$

Only the mated adult mortality rates $\mu_{m 2 c}$ and $\mu_{f 2 c}$ are increased by the costs of reproduction. Let this increase be linearly proportional to the reproductive costs $E_{m}$ and $E_{f}$, so that the mortality rates of mated adults are:

$$
\begin{gathered}
\mu_{m 2 c}=\mu_{m 2}+c E_{m} \\
\mu_{f 2 c}=\mu_{f 2}+c E_{f}
\end{gathered}
$$

where $c$ is a nonnegative constant. We will assume that the baseline male and female mortality rates are equal - that is, $\mu_{m 1}=\mu_{f 1}$ and $\mu_{m 2}=\mu_{f 2}$ - so the sexes only differ in how they affect parental survival through $D_{m}$ and $D_{f}$.

Charnov (1982, Chapter 6) considers a case where mothers experience higher annual mortality rates when having sons instead of daughters. He states that the sex ratio will be biased towards sons so that:

$$
\frac{s_{1}}{1-s_{1}}=\frac{\text { maternal mortality for rearing a daughter }}{\text { maternal mortality for rearing a son }}
$$

This is equivalent to (3.1), when offspring costs are framed in terms of a parental mortality expense.

In qualitative agreement with Charvnov's predictions, $s_{1}^{*}$ in our model favors the sex that induces less parental mortality (Figure 3-7a). This implies that evolution may favor the preservation of already breeding adults, rather than having them die producing new offspring. Favoring the sex that induces less parental mortality also reduces union dissolution due to partner death. 


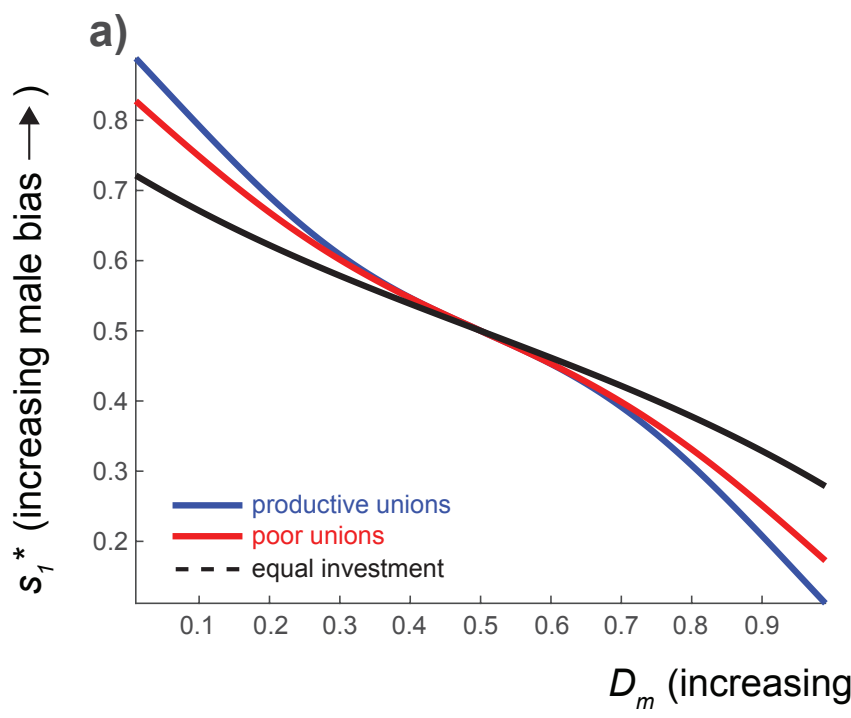

b)

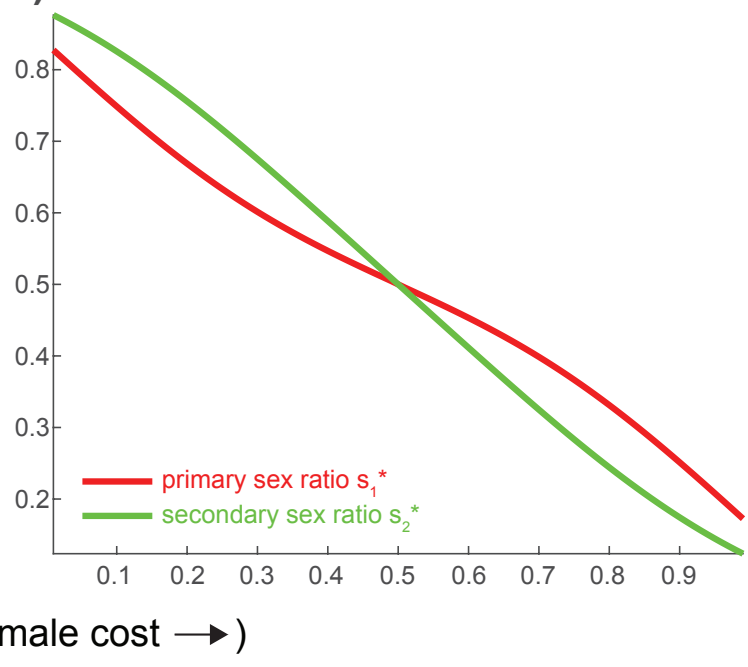

Figure 3-7: Case 4 singular strategy (SS) sex ratios, as a function of male cost on parental survival $D_{m}$ in (3.42), with $c=0.1$. Wet set the female cost $D_{f}=1-D_{m}$ (if male offspring impose more parental mortality, female offspring impose less parental mortality, and vice versa). a) The primary sex ratio $s_{1}^{*}$ for productive unions (blue), poor unions (red), and poor unions with strong juveniles (black). Strong juveniles have lower mortality rates $\left(\mu_{m 1}=\mu_{f 1}=0.01\right)$ and faster maturation rates $\left(\alpha_{m}=\alpha_{f}=5\right)$. b) The corresponding secondary sex ratio $s_{2}^{*}$ (green) in the poor unions case.

In contrast to Cases 1-3, productive unions (Figure 3-7a, blue) have more sex ratio bias than poor unions - likely because adults with greater reproductive output also have greater parental mortality. However, "strong juveniles" with lower juvenile mortality and higher maturation rates (Figure 3-7a, black) reduce the sex ratio bias. In this case, newborn juveniles are faster, more viable replacements for their parents, thereby alleviating the costs of parental death.

Because parental mortality only affects adults, it occurs after the period of parental investment. Thus, as in Case 3, the secondary sex ratio is even more biased towards the cheaper sex than the primary sex ratio is (Figure $3-7 \mathrm{~b}$ ).

\subsubsection{Evolutionary and convergence stability of the SS sex ratio}

The evolutionary and convergence stability properties of the SS sex ratio $s_{1}^{*}$ are identical in Cases 1-4. In all four cases, the second derivative expression (3.22) is approximately zero and (3.34) is negative (examples in Figure 3-8). Thus, all the singular strategies we have observed are convergence stable "weak form" ESSs, as we previously encountered when male and female offspring were identically costly (Case 0, Section 3.4.1).

As a result, $s_{1}^{*}$ is an evolutionary attractor to which that populations will ultimately converge through a series of small mutations. Once the resident population is at $s_{1}^{*}$, any mutant sex ratio will have the same fitness as the resident. However, since the invasion fitness (3.17) is zero rather than positive, it will not displace the resident through natural selection. Though there is no selection for 
a)

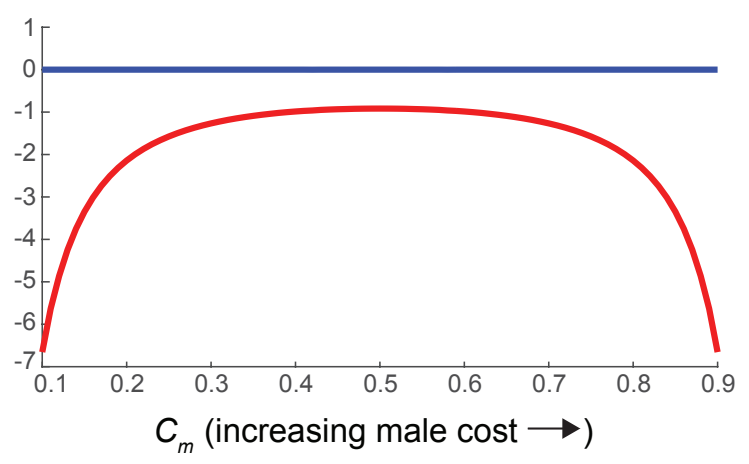

b)

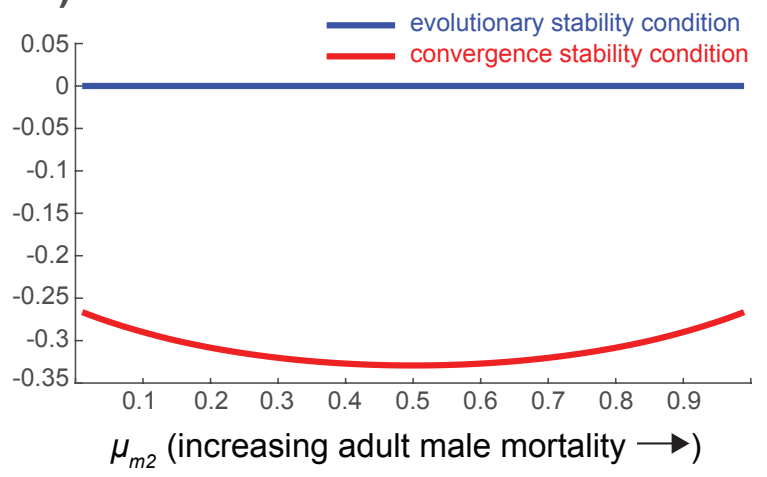

Figure 3-8: Values of the evolutionary stability condition (3.22) and convergence stability condition (3.34) over a range of offspring costs. a) Case 1, productive unions. b) Case 3 (adult mortality), poor unions.

a new phenotype at a neutral ESS, different rare sex ratios could potentially arise via neutral drift (which may be a mechanism for generating genetic diversity), and even small deviations might shift selective pressures (Bull and Charnov 1988).

The selective neutrality of certain sex ratios has also been noted in models without demographic structure. In Shaw-Mohler fitness formulation (3.2), for instance, all individual sex ratios have the same fitness when the population sex ratio is 0.5. Our results suggest that selectively neutral, convergence stable SS sex ratios may be integral features of two-sex systems in general, as they are maintained even in models with more complex population structure, and consistently appear over a wide range of offspring cost interpretations and values.

\subsubsection{Primary reproductive value ratios}

Instead of considering the relative abundances of each sex, as given by the sex ratio, one can also consider their relative reproductive values.

Fisher (1930) originally stated that the total reproductive value of each sex in a given generation must be equal. This notion of reproductive value has been invoked in various ways in studies of sex ratio. Some (e.g., Bodmer and Edwards 1960) specifically consider genetic contributions to grandchildren, so that an individual's reproductive value is inversely proportional to the total surviving individuals of their sex. Others (e.g., Grafen 2014) define an individual's reproductive value as the probability that a random future gene can be traced back to that individual.

We will consider the lifetime reproductive value for each population stage as follows. In a matrix model, the dominant left eigenvector $\mathbf{v}$ of the projection matrix $\mathbf{A}$ is a vector of stage-specific reproductive values (shown in age-structured models by Goodman 1968; extended to stage-structured models by Taylor 1990). 
Recall that the selection gradient (3.19) depends on $\mathbf{v}^{\prime}$ as follows:

$$
\frac{d \lambda^{\prime}}{d s_{1}^{\prime}}=\left(\mathbf{w}^{\prime \top} \otimes \mathbf{v}^{\prime \top}\right) \frac{d \mathrm{vec} \mathbf{A}^{\prime}}{d s_{1}^{\prime}}
$$

For a $s \times 1$ population vector, the Kronecker product in (3.46) is the $1 \times s^{2}$ vector:

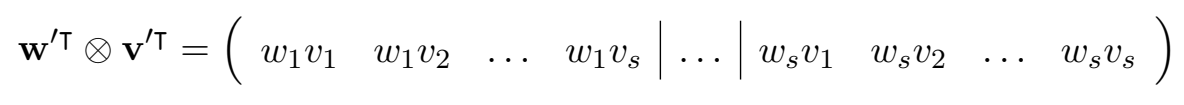

where $w_{i}$ is the $\mathrm{i}^{\text {th }}$ entry of $\mathbf{w}^{\prime}$ (stable stage frequency of stage $i$ ), and $v_{i}$ is the $\mathrm{i}^{\text {th }}$ entry of $\mathbf{v}^{\prime}$ (reproductive value of stage $i$ ).

At any singular strategy sex ratio $s_{1}^{*}$, the selection gradient (3.46) is equal to 0. Substituting (3.47) into (3.46) and evaluating at $s_{1}^{*}$, we obtain:

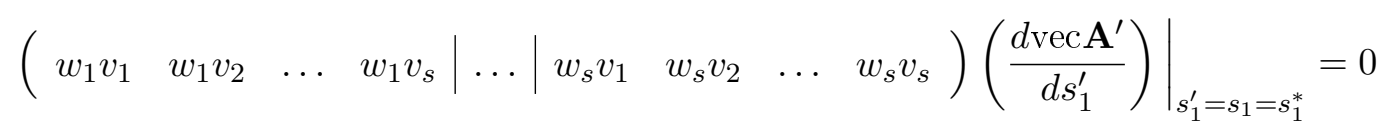

where

$$
\frac{d \mathrm{vec} \mathbf{A}^{\prime}}{d s_{1}^{\prime}}=\frac{1}{3}\left(\frac{d \mathrm{vec} \mathbf{T}^{\prime}}{d s_{1}^{\prime}}+\frac{d \mathrm{vec} \mathbf{B}^{\prime}}{d s_{1}^{\prime}}+\frac{d \mathrm{vec} \mathbf{U}^{\prime}}{d s_{1}^{\prime}}\right)
$$

\subsubsection{Case 1 and 2}

In Case 1 (offspring resource cost, Section 3.4.2), $\mathbf{B}^{\prime}$ is a function of $s_{1}^{\prime}$, but $\mathbf{U}^{\prime}$ and $\mathbf{T}^{\prime}$ are not. Thus, (3.49) simplifies to:

$$
\frac{d \mathrm{vec} \mathbf{A}^{\prime}}{d s_{1}^{\prime}}=\frac{1}{3} \frac{d \mathrm{vec} \mathbf{B}^{\prime}}{d s_{1}^{\prime}}
$$

The matrix $\mathbf{B}$ is given by (3.7) so that:

$$
\begin{aligned}
\operatorname{vec} \mathbf{B}^{\prime} & =\left(\begin{array}{lllllll}
0 & \ldots & \frac{R s_{1}^{\prime}}{C_{a}} & 0 & \frac{R\left(1-s_{1}^{\prime}\right)}{C_{a}^{\prime}} & 0 & 0
\end{array}\right)^{\top} \\
\frac{d \operatorname{vec} \mathbf{B}^{\prime}}{d s_{1}^{\prime}} & =\left(\begin{array}{lllllll}
0 & \ldots & \frac{R C_{f}}{C_{a}^{2}} & 0 & \frac{-R C_{m}}{C_{a}^{\prime 2}} & 0 & 0
\end{array}\right)^{\top}
\end{aligned}
$$

where $C_{a}$ is given by $(3.35)$.

Substituting (3.51) into (3.50), then into (3.48), we obtain:

$$
\frac{C_{f} R}{C_{a}^{2}} w_{s} v_{1}-\frac{C_{m} R}{C_{a}^{2}} w_{s} v_{3}=0
$$

Canceling out terms and rearranging, we obtain the simple expression:

$$
C_{f} v_{1}=C_{m} v_{3}
$$


Again, $v_{i}$ corresponds to the reproductive value of stage $i$. In our population vector (3.3), stage 1 is $m_{1}$ (juvenile males), and stage 3 is $f_{1}$ (juvenile females). Thus, (3.53) becomes:

$$
\frac{v_{m 1}}{v_{f 1}}=\frac{C_{m}}{C_{f}}
$$

The expression (3.54) shows that, at $s_{1}^{*}$, the primary reproductive value ratio $\frac{v_{m 1}}{v_{f 1}}$ (ratio of juvenile male to juvenile female reproductive values) equals the ratio of the sex-specific resource costs. This expression is analogous to the inverse of the equal investment principle (3.1), but is written in terms of the reproductive value ratio rather than the sex ratio.

The same result (3.54) holds for Case 2 (offspring mortality during parental investment, Section 3.4.3), if $C_{m}$ and $C_{f}$ are given by (3.41).

\subsubsection{Case 3}

In Case 3 (offspring mortality after parental investment, Section 3.4.4), (3.49) once again simplifies to (3.50). The matrix $\mathbf{B}$ is given by (3.7) so that:

$$
\begin{aligned}
\operatorname{vec}^{\prime} & =\left(\begin{array}{lllllll}
0 & \ldots & R s_{1}^{\prime} & 0 & R\left(1-s_{1}^{\prime}\right) & 0 & 0
\end{array}\right)^{\top} \\
\frac{d \operatorname{vec}^{\prime}}{d s_{1}^{\prime}} & =\left(\begin{array}{lllllll}
0 & \ldots & R & 0 & -R & 0 & 0
\end{array}\right)^{\top}
\end{aligned}
$$

Substituting (3.55) into (3.50), then into (3.48), we obtain:

$$
R w_{s} v_{1}-R w_{s} v_{3}=0
$$

which reduces to

$$
v_{m 1}=v_{f 1}
$$

In other words, the reproductive values of juvenile males $v_{m 1}$ and juvenile females $v_{f 1}$ are equal at $s_{1}^{*}$. The corresponding primary reproductive value ratio $\frac{v_{m 1}}{v_{f 1}}$ is thus 0.5 regardless of sex-specific mortality.

\subsubsection{Case 4}

In Case 4 (parental mortality cost, Section 3.4.5), both $\mathbf{B}^{\prime}(3.15)$ and $\mathbf{T}^{\prime}(3.43)$ are functions of $s_{1}^{\prime}$, so (3.49) becomes:

$$
\frac{d \mathrm{vec} \mathbf{A}^{\prime}}{d s_{1}^{\prime}}=\frac{1}{3}\left(\frac{d \mathrm{vec} \mathbf{B}^{\prime}}{d s_{1}^{\prime}}+\frac{d \mathrm{vec} \mathbf{T}^{\prime}}{d s_{1}^{\prime}}\right)
$$

After differentiating and performing several algebraic manipulations, (3.48) yields the expression:

$$
v_{m 1}=v_{f 1}+c\left[\left(D_{f}-D_{m}\right)\left(U_{f} v_{m 2}+U_{m} v_{f 2}-\left(U_{f}+U_{m}\right) v_{u}\right)\right]
$$


In this case, the relationship between reproductive values is more complex. The amount by which $v_{m 1}$ deviates from $v_{f 1}$ is determined by the mortality effect $c$. In the limit as $c \rightarrow 0, v_{m 1} \rightarrow v_{f 1}$, as in Case 3.

\subsection{Discussion}

Because reproduction is shaped by the entire life cycle, and stage-specific offspring costs are often speculated to affect sex ratios, demographic population models lend additional insight into sex ratio theory. We have shown how to formulate flexible demographic two-sex models, and how to perform evolutionary analyses of these models using adaptive dynamics. Our analyses include calculations and characterizations of singular strategies that depend on sex and stage differences, demonstrating how demographic considerations affect evolution.

Using this approach, we found that four alternative interpretations of sex-biased offspring costs may modify the primary sex ratio (Table 3.1 ). In some cases, our results contradict the widely-held conclusions of models that neglect demographic population structure, most notably the classic belief that mortality after the period of parental investment cannot affect the primary sex ratio.

\subsubsection{The importance of union formation}

Our contradictory results may arise from our incorporation of a union stage. Two-sex models that do not include unions allow adults to reproduce directly, and thus do not distinguish between the "mating advantage" and "offspring production advantage" of the rarer (e.g., higher-mortality) sex.

Figure 3-1 compares the general structure of models with and without unions. A "mating advantage" increases the rate at which singles form unions (highlighted red arrow), while a "offspring production advantage" increases the rate at which singles ultimately produce offspring.

In models without unions, single males and females produce offspring directly. In these models, the birth rate is often proportional to the mating function (e.g., Caswell and Weeks 1986, Hardy 2002), so the rarer (higher-mortality) sex will have greater fertility and produce more offspring. The directly increased "offspring production advantage" of this higher-mortality sex appears to counterbalance its mortality and maintain $s_{1}^{*}$ at equality.

In models with unions, singles must first enter the union stage to produce offspring. Single adults enter unions at rates given by the mating functions, and may return to the singles stages due to union dissolution from divorce or partner mortality. In this case, the rare, higher-mortality sex will have greater mating rates, which may increase its offspring production indirectly. However, this "mating advantage" of the rarer sex is not always proportional to its ultimate "offspring production advantage." If unions are poor, due to low resource investment rate $R$ or high divorce $d$, they ultimately may not produce many offspring. The "offspring production advantage" of the rarer (higher-mortality) sex may thus be reduced, causing $s_{1}^{*}$ to favor the more common (lower-mortality) sex. 
model with unions

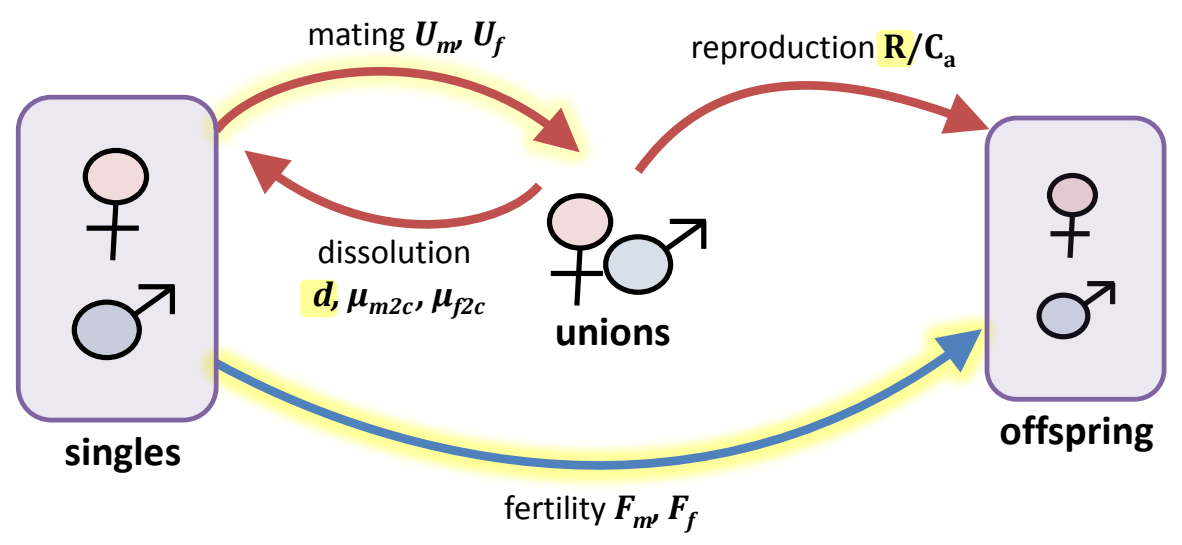

model without unions

Figure 3-1: A comparison of two-sex models with unions (top, red arrows) and without unions (bottom, blue arrow). Parameters used as indicators of union productivity (the divorce rate $d$ and resource investment rate $R$ ) are highlighted in yellow. The highlighted arrows indicate the transitions increased by the "mating advantage" (red highlighted arrow) and "offspring production advantage" (blue highlighted arrow) of the rarer sex.

As an illustrative example of a model without unions, consider a 4-stage model that allows all adults to produce offspring directly. This population contains non-breeding (juvenile) males $m_{1}$ and females $f_{1}$ that mature into breeding (adult) males $m_{2}$ and females $f_{2}$, which then produce new offspring (Figure 3-2).

The 4-stage population vector is:

$$
\mathbf{n}(t)=\left(\begin{array}{c}
m_{1} \\
m_{2} \\
f_{1} \\
f_{2}
\end{array}\right)
$$

We now use birth rates rather than mating rates. Assume, as in Case 3, that all offspring resource costs are normalized to $1\left(C_{m}=C_{f}=C_{a}=1\right)$. The total birth rate $B(\mathbf{n})$ is the product of the resource investment rate $R$ and the total mating function $M(\mathbf{n})$ from (3.4):

$$
B(\mathbf{n})=R M(\mathbf{n})
$$

The corresponding per capita male and female fertility rates are:

$$
\begin{aligned}
F_{m}(\mathbf{n}) & =\frac{B(\mathbf{n})}{2 m} \\
F_{f}(\mathbf{n}) & =\frac{B(\mathbf{n})}{2 f}
\end{aligned}
$$




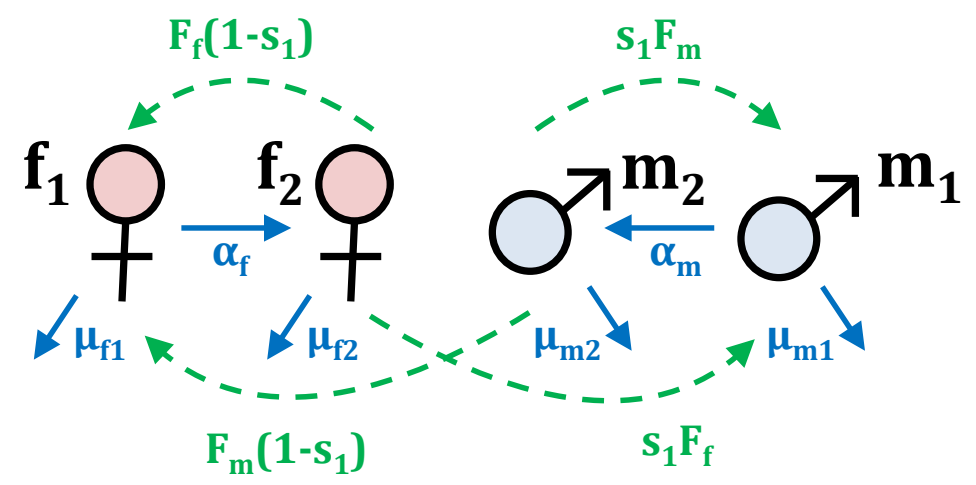

Figure 3-2: Life cycle diagram for a 4-stage population with juvenile males $m_{1}$ and juvenile females $f_{1}$, and adult males $m_{2}$ and adult females $f_{2}$. The functions and parameters shown here appear in the birth matrix $\mathbf{B}$ (3.64) (green), or transition matrix $\mathbf{T}$ (3.65) (blue).

where the factor of $\frac{1}{2}$ prevents double-counting offspring from both males and females.

Because we have eliminated the mating process, the mating matrix $\mathbf{U}$ is simply a matrix of zeros. The birth and transition rate matrices are now:

$$
\begin{aligned}
\mathbf{B}(\mathbf{n}) & =\left(\begin{array}{cccc}
0 & s_{1} F_{m} & 0 & s_{1} F_{f} \\
0 & 0 & 0 & 0 \\
0 & \left(1-s_{1}\right) F_{m} & 0 & \left(1-s_{1}\right) F_{f} \\
0 & 0 & 0 & 0
\end{array}\right) \\
\mathbf{T} & =\left(\begin{array}{cccc}
-\left(\mu_{m 1}+\alpha_{m}\right) & 0 & 0 & 0 \\
\alpha_{m} & -\mu_{m 2} & 0 & 0 \\
0 & 0 & -\left(\mu_{f 1}+\alpha_{f}\right) & 0 \\
0 & 0 & \alpha_{f} & -\mu_{f 2}
\end{array}\right)
\end{aligned}
$$

As in Case 3, we will consider sex-biased mortality after parental investment. As shown in Figure $3-3$, the equal sex ratio $s_{1}^{*}=0.5$ is now preserved for both juvenile and adult mortality. In the 4-stage model without unions, higher mortality in one sex appears to be fully compensated for by its higher fertility.

\subsubsection{The role of reproductive value}

We have found that several well-known predictions about the primary sex ratio are actually more applicable to the primary reproductive value ratio. This includes the equal investment principle (3.1), and the claim that mortality after parental investment cannot bias the sex ratio. Although we found deviations from the sex ratios predicted by the equal investment principle in Case 1 (Section 3.4.2) and Case 2 (Section 3.4.3), (3.54) shows that an analogous principle still holds for the reproductive value ratio instead (Figure $3-4 \mathrm{a}, \mathrm{b}$ ). We also found that mortality after parental 

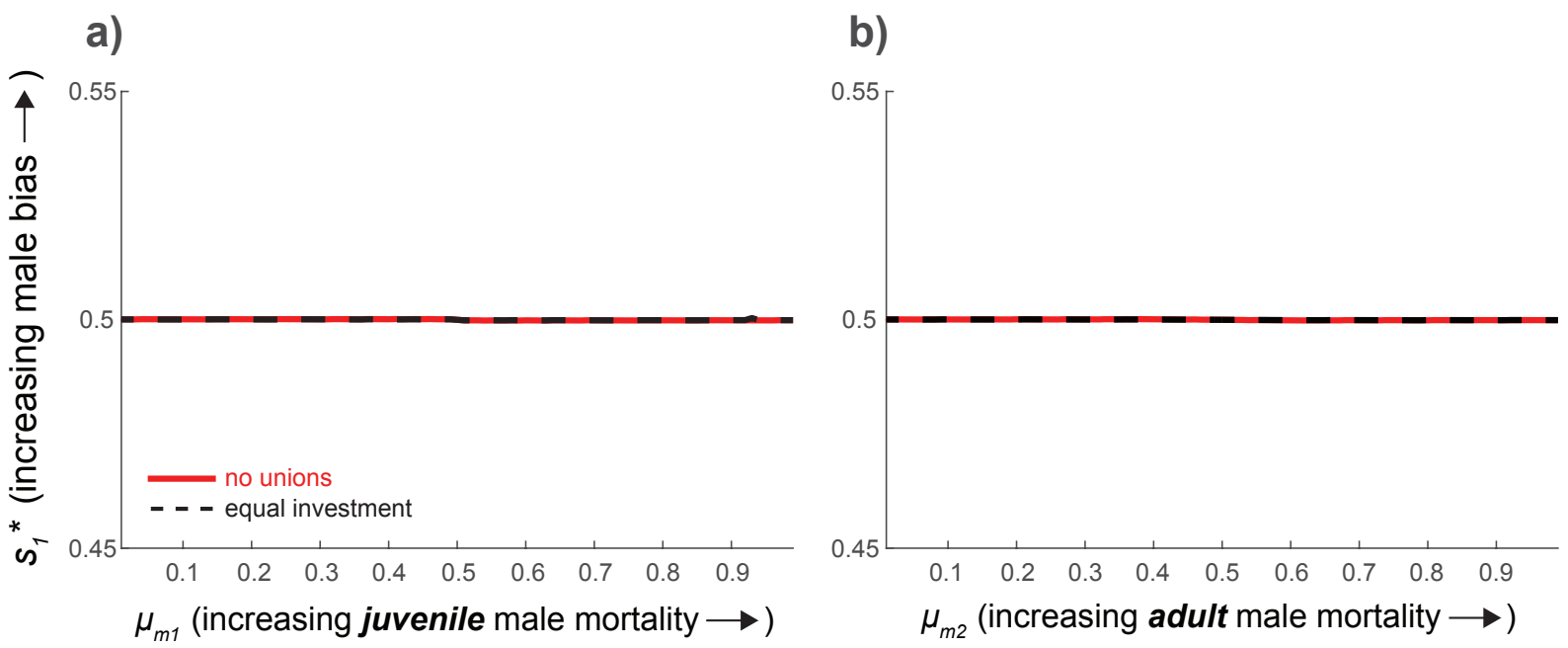

Figure 3-3: Singular strategy (SS) sex ratios for the 4-stage (no unions) model, as a function of the a) juvenile male mortality rate $\mu_{m 1}$, with juvenile female mortality $\mu_{f 1}=1-\mu_{m 1}$, or the $b$ ) adult male mortality rate $\mu_{m 2}$, with adult female mortality $\mu_{f 2}=1-\mu_{m 2}$.

investment can bias the SS sex ratio (Case 3, Section 3.4.4), but it cannot bias the corresponding reproductive value ratio by $(3.57)$ (Figure $3-4 \mathrm{c}, \mathrm{d}$ ).

Consequently, we would only expect the primary sex ratio to follow an equal investment principle and be unaffected by mortality after investment if it were equal to the primary reproductive value ratio - that is, if the lifetime contribution of each sex to future generations was directly proportional to its relative abundance at birth.

However, the primary sex ratio and reproductive value ratios appear to deviate in our 5-stage model, especially when unions are poor. If unions are unproductive, sex-specific reproductive values may be differentially reduced, with the rarer sex having much less of a reproductive advantage. The rarer sex must thus become even rarer to raise its reproductive value to the same level as the more common sex, biasing the primary sex ratio in favor of the more common sex.

\subsubsection{Extensions and caveats}

We have focused on four common interpretations of sex-biased offspring costs, but there are many additional sex-specific differences that can affect the sex ratio, which could studied by the appropriate addition of population stages or rate matrices to our demographic model. For example, male and female offspring may differ not only in how they affect parental survival, but also in how they affect future parental reproduction. Female red deer, for instance, settle closer to their parents than males do, increasing mate competition (Trivers 1985).

Offspring may also benefit their parents through sex-specific cooperation. In some cooperatively breeding birds, for instance, young males stay with their parents for several years to help rear new 
a) Case 1

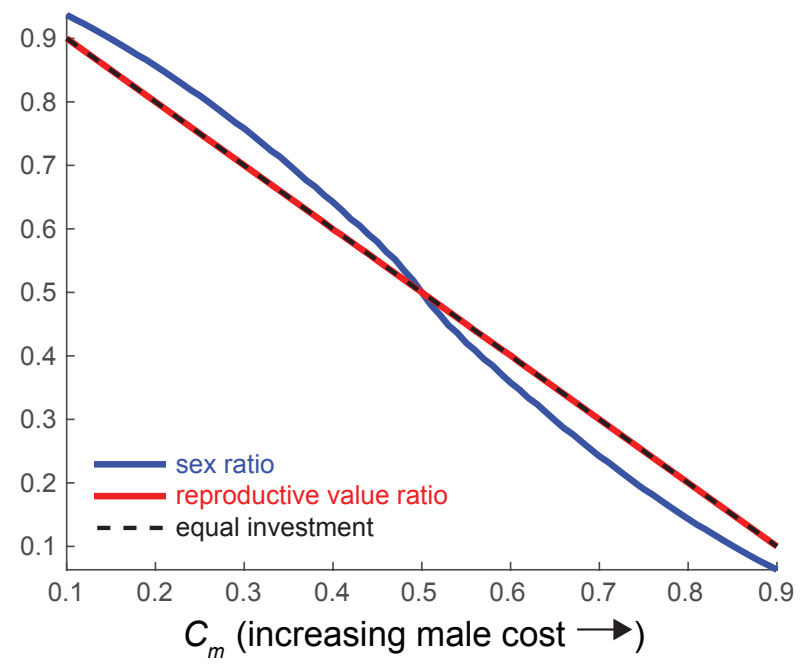

c) Case 3 (juvenile mortality)

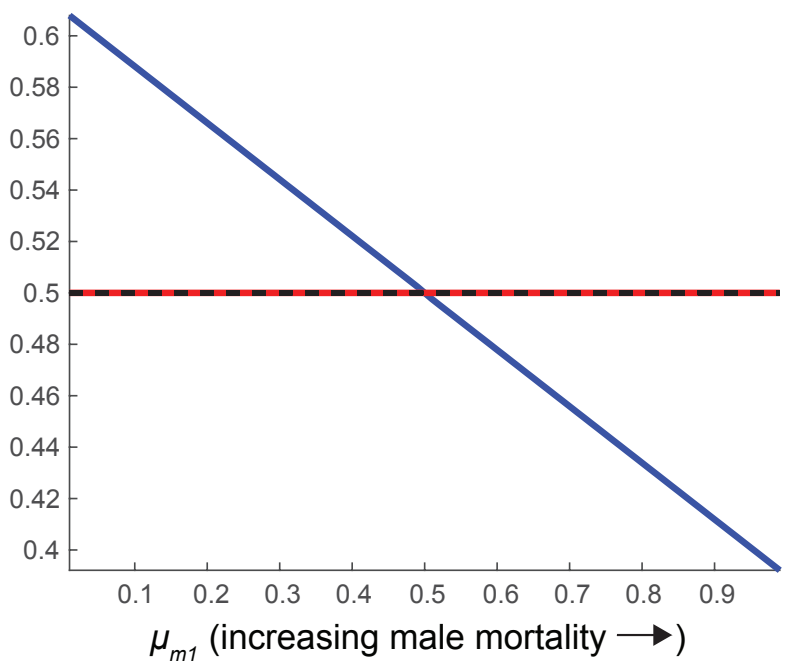

b) Case 2

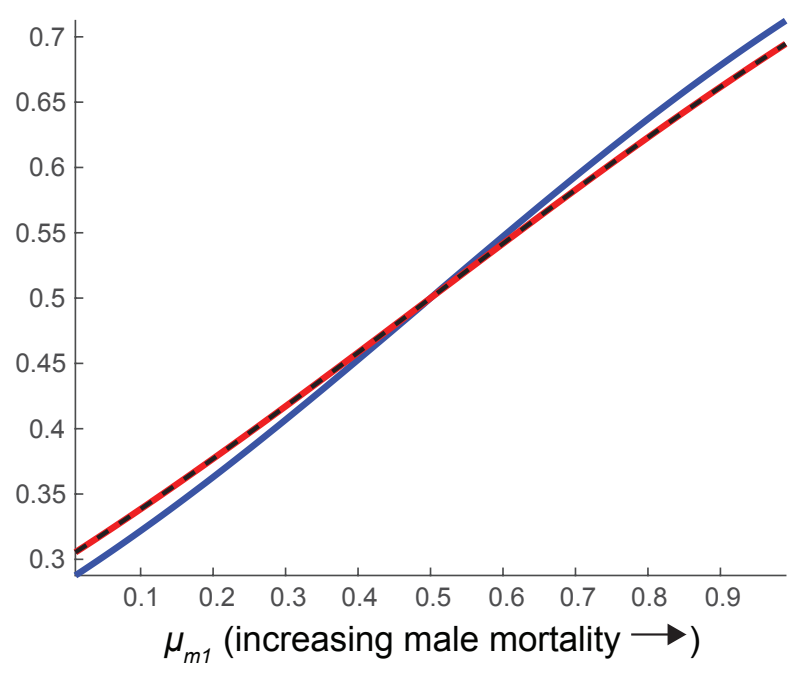

d) Case 3 (adult mortality)

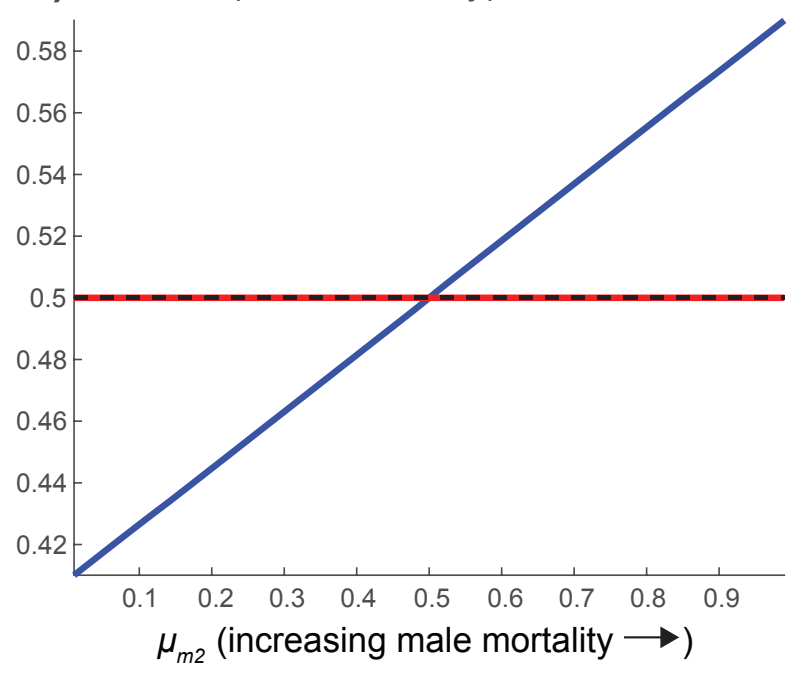

Figure 3-4: A comparison of the primary sex ratios (blue) and reproductive value ratios (red) with the equal investment principle ratio (3.1) (black). In a) Case 1 (Section 3.4.2) and b) Case 2 (Section 3.4.3), the primary reproductive value ratio is given by the inverse of (3.54). In $c$ ) Case 3, juvenile mortality (Section 3.4.4.1) and $d$ ) Case 3, adult mortality (Section 3.4.4.2), the primary reproductive value ratio is always 0.5 by (3.57). Parameters for poor unions (Table 3.2) were used in all cases. 
broods. This may cause the sex ratio to favor the more "helpful" sex, as evidenced by male-biased fledgling ratios in woodpeckers (Frank 1990).

We also note that there is often not a single, fixed limiting resource for offspring production (Frank 1990), as we have assumed in Cases 1 and 2. The offspring costs or gains per unit of parental investment are not always fixed as well, and may vary according to a nonlinear returns model (Charnov 1979b). These factors may cause additional sex ratio biases that were not noted here. The evolutionary effects of these other considerations, or multiple costs acting simultaneously, could likely be modeled using a similar approach.

\subsection{Acknowledgments}

This work was supported by a National Science Foundation Graduate Research Fellowship under

Grant 1122374, NSF Grants DEB1145017 and DEB1257545 (to H. Caswell), Advanced Grant 322989 from the European Research Council (to H. Caswell), and the Woods Hole Oceanographic Institution Academic Programs Office.

\section{Bibliography}

[1] Bull JJ, Charnov EL. 1988. How fundamental are Fisherian sex ratios? Oxford Surveys in Evolutionary Biology. 5: 96-135.

[2] Caswell H, Weeks DE. 1986. Two-sex models: chaos, extinction, and other dynamic consequences of sex. American Naturalist. 128: 707-735.

[3] Caswell H. 2001. Matrix population models. Sunderland: Sinauer Associates, Inc.

[4] Caswell H. 2008. Perturbation analysis of nonlinear matrix models. Demographic Research. 18: 59-116.

[5] Caswell H. 2010. Reproductive value, the stable stage distribution, and the sensitivity of the population growth rate to changes in vital rates. Demographic Research. 23:531-548.

[6] Charlesworth B. 1977. Population genetics, demography, and the sex ratio. Measuring Selection in Natural Populations. Lecture Notes in Biomathematics. 19: 345-363.

[7] Charnov EL. 1975. Sex ratio selection in an age-structured population. Evolution. 29: 366-368.

[8] Charnov EL. 1979. The genetical evolution of patterns of sexuality: Darwinian fitness. American Naturalist. 113: 465-480.

[9] Charnov EL. 1979. Simultaneous hermaphroditism and sexual selection. Proc. Natl. Acad. Sci. USA. 76: 2480-2484.

[10] Charnov EL. 1982. The theory of sex allocation. Princeton: Princeton University Press.

[11] Darwin C. 1871. The Descent of Man and Selection in Relation to Sex. London: John Murray. 
[12] Darwin C. 1874. The Descent of Man and Selection in Relation to Sex. 2nd edition. London: John Murray.

[13] Dieckmann U, Law R. 1996. The dynamical theory of coevolution: a derivation from stochastic ecological processes. Journal of Mathematical Biology. 34: 579-612.

[14] Düsing, C. 1883. Die Factoren welche die Sexualität entscheide. Jenaische Zeitschrift für Naturwissenschaft. 16, 428-464.

[15] Edwards AWF. 2000. Carl Düsing (1884) on The regulation of the sex-ratio. Theoretical Population Biology. 58: 255-257.

[16] Emlen JM. 1968a. A note on natural selection and the sex ratio. American Naturalist. 102: 94-95.

[17] Emlen JM. 1968b. Selection for the sex ratio. American Naturalist. 102: 589-591.

[18] Eshel I. 1975. Selection on sex-ratio and the evolution of sex-determination. Heredity. 34: 351-361.

[19] Eshel I. 1983. Evolutionary and continuous stability. Journal of Theoretical Biology. 103: 99-111.

[20] Fisher RA. 1930. The Genetical Theory of Natural Selection. Oxford: Oxford University Press.

[21] Frank SA. 1990. Sex allocation theory for birds and mammals. Annual Review of Ecology and Systematics. 21: 13-55.

[22] Geritz SAH, Kidsi É, Meszéna G, Metz JAJ. 1998. Evolutionarily singular strategies and the adaptive growth and branching of the evolutionary tree. Evolutionary Ecology. 12: 35-57.

[23] Goodman LA. 1968. An elementary approach to the population projection-matrix, to the population reproductive value, and to related topics in the mathematical theory of population growth. Demography. 5: 382-409.

[24] Grafen A. 2014. Total reproductive values for females and for males in sexual diploids are not equal. Journal of Theoretical Biology. 359: 233-235.

[25] Hadeler KP, Waldstätter R, Wörz-Busekros A. 1988. Models for pair formation in bisexual populations. Journal of Mathematical Biology. 26: 635-649.

[26] Hadeler KP. 1989. Pair formation in age-structured populations. Acta Applicandae Mathematicae. 14: 91-102.

[27] Hamilton WD. 1967. Extraordinary sex ratios. Science. 156: 477-488.

[28] Hardy, ICW. 2002. Sex ratios: concepts and methods. Cambridge University Press, Cambridge.

[29] Helle S, Lummaa V, Jokela J. 2002. Sons reduced maternal longevity in preindustrial humans. Science. 296: 1085.

[30] Iannelli, M, Martcheva M, Milner F. 2005. Gender-structured population modeling: mathematical methods, numerics, and simulations. Philadelphia: Society for Industrial and Applied Mathematics. 
[31] Kahn AT, Jennions MD, Kokko H. 2015. Sex allocation, juvenile mortality and the costs imposed by offspring on parents and siblings. Journal of Evolutionary Biology. 28: 428-437.

[32] Karlin S, Lessard S. 1986. Theoretical Studies on Sex Ratio Evolution. Princeton: Princeton University Press.

[33] Kolman WA. 1960. The mechanism of natural selection for the sex ratio. American Naturalist. 94: $373-377$.

[34] Maynard Smith J, Price GR. 1973. The logic of animal conflict. Nature. 246: 15-18.

[35] Merrell DJ. 1981. Ecological Genetics. Minneapolis: University of Minnesota Press.

[36] Metz JAJ. 2006. Fitness. IIASA Interim Report IR-06-061. In Encyclopedia of Ecology (ed Jørgensen SE), pp.1599-1612, Elsevier (2008).

[37] Metz JAJ, Nisbet RM, Geritz SAH. 1992. How should we define 'fitness' for ecological scenarios? TREE. 7: 198-202.

[38] Otto SP, Day T. 2007. A biologist's guide to mathematical modeling in ecology and evolution. Princeton: Princeton University Press.

[39] Pollak RA. 1986. A reformulation of the two-sex problem. Demography. 23: 247-259.

[40] Shaw RF, Mohler JD. 1953. The selective significance of the sex ratio. American Naturalist. 87: $337-342$.

[41] Shyu E, Caswell H. 2014. Calculating second derivatives of population growth rates for ecology and evolution. Methods in Ecology and Evolution. 5: 473-482.

[42] Shyu E, Caswell H. xxa. A two-sex demographic matrix model formulation of the birth matrix-mating rule. In preparation.

[43] Shyu E, Caswell H. xxb. The evolution of facultative sex ratios with two maternal conditions. In preparation.

[44] Slagsvold T, Røskaft E, Engen S. 1986. Sex ratio, differential cost of rearing young, and differential mortality between the sexes during the period of parental case: Fisher's theory applied to birds. Ornis Scandinavica. 17: 117-125.

[45] Taylor PD. 1990. Allele-frequency change in a class-structured population. American Naturalist. 135: 95-106.

[46] Trivers RL. 1972. Parental investment and sexual selection. In B. Campbell (Ed.), Sexual selection and the descent of man, 1871-1971. Chicago: Aldine Publishing Company.

[47] Trivers RL. 1974. Parent-offspring conflict. American Zoologist. 14: 249-264.

[48] Trivers RL, Hare H. 1976. Haploidploidy and the evolution of the social insect. Science. 191: 249-263.

[49] Trivers RL, Willard DE. 1973. Natural selection of parental ability to vary the sex ratio of offspring. Science. 179: 90-92. 
[50] Trivers RL. 1985. The primary sex ratio. In Social Evolution. Menlo Park: Benjamin-Cummings Publishing Company.

[51] Uyenoyama MK, Bengtsson BO. 1979. Towards a genetic theory for the evolution of the sex ratio. Genetics. 93: 721-736.

[52] Uyenoyama MK, Bengtsson BO. 1982 Towards a genetic theory for the evolution of the sex ratio III. Parental and sibling control of brood investment ratio under partial sib-mating. Theoretical Population Biology. 22: 43-68.

[53] Weimerskirch H, Lallemand J, Martin J. 2005. Population sex ratio variation in a monogamous long-lived bird, the wandering albatross. Journal of Animal Ecology. 74: 285-291.

[54] West SA. 2009. Sex allocation. Princeton: Princeton University Press.

[55] Wisser O, Vaupel JW. 2014. The sex differential in mortality: a historical comparison of the adult-age pattern of the ratio and the difference. MPIDR Working Paper WP-2014-005. 


\section{Chapter 3 Appendix}

\section{A Relationship between second derivatives at singular strategies}

Following Geritz et al. (1998), we note that $s_{x}(y)=0$ at any singular strategy $y=x=x^{*}$ i.e., if the mutant and resident phenotypes are identical, the invasion fitness is 0 . The directional derivative of $s_{x}(y)$ along $y=x, D_{y=x}\left[s_{x}(y)\right]$, is thus also 0 :

$$
\begin{aligned}
D_{y=x}\left[s_{x}(y)\right] & =\frac{\partial s_{x}(y)}{d x}+\frac{\partial s_{x}(y)}{d y} \\
& =0 \quad \text { when } y=x=x^{*}
\end{aligned}
$$

Similarly, the second-order directional derivative of $s_{x}(y)$ along $y=x$ must also be 0 :

$$
\begin{aligned}
\left.D_{y=x}\left(D_{y=x}\left[s_{x}(y)\right]\right)\right|_{y=x=x^{*}} & =\frac{\partial}{\partial x}\left(\frac{\partial s_{x}(y)}{d x}+\frac{\partial s_{x}(y)}{d y}\right)+\frac{\partial}{\partial y}\left(\frac{\partial s_{x}(y)}{d x}+\frac{\partial s_{x}(y)}{d y}\right) \\
& =\frac{\partial^{2} s_{x}(y)}{\partial y^{2}}+2 \frac{\partial^{2} s_{x}(y)}{\partial x \partial y}+\frac{\partial^{2} s_{x}(y)}{\partial^{2} x} \\
& =0 \quad \text { when } y=x=x^{*}
\end{aligned}
$$

Assuming that $s_{x}(y)$ is twice continuously differentiable, the following relationship holds at any singular strategy $x^{*}$ :

$$
\left.\left(\frac{\partial^{2} s_{x}(y)}{\partial y^{2}}+2 \frac{\partial^{2} s_{x}(y)}{\partial x \partial y}+\frac{\partial^{2} s_{x}(y)}{\partial^{2} x}\right)\right|_{y=x=x^{*}}=0
$$

In the two-sex matrix model, (3.68) becomes (3.31).

\section{B Calculating mixed second derivatives}

As in Shyu and Caswell (2014), the pure second derivatives of $\lambda^{\prime}$ with respect to $\boldsymbol{\theta}^{\prime}$ are:

$$
\frac{\partial^{2} \lambda^{\prime}}{\partial \boldsymbol{\theta}^{\prime} \partial \boldsymbol{\theta}^{\prime \top}}=\frac{d}{d \boldsymbol{\theta}^{\prime \top} \mathrm{vec}}\left[\left(\frac{d \tilde{\lambda}}{d \boldsymbol{\theta}^{\prime \top}}\right)^{\top}\right]
$$


The mixed second derivatives of $\lambda^{\prime}$ with respect to $\boldsymbol{\theta}^{\prime}$ and $\boldsymbol{\theta}$ are similarly:

$$
\frac{\partial^{2} \lambda^{\prime}}{\partial \boldsymbol{\theta} \partial \boldsymbol{\theta}^{\prime \top}}=\frac{d}{d \boldsymbol{\theta}^{\top}} \operatorname{vec}\left[\left(\frac{d \tilde{\lambda}}{d \boldsymbol{\theta}^{\prime \top}}\right)^{\top}\right]
$$

It can be shown (Caswell 2010) that:

$$
\frac{d \tilde{\lambda}}{d \boldsymbol{\theta}^{\prime \top}}=\left(\mathbf{w}^{\prime} \otimes \mathbf{v}^{\prime}\right)^{\top} \frac{d \mathrm{vec} \mathbf{A}^{\prime}}{d \boldsymbol{\theta}^{\prime \top}}
$$

Substituting (3.71) into (3.70), we obtain:

$$
\frac{\partial^{2} \lambda^{\prime}}{\partial \boldsymbol{\theta} \partial \boldsymbol{\theta}^{\prime \top}}=\frac{d}{d \boldsymbol{\theta}^{\top}} \operatorname{vec}\left[\left(\frac{d \mathrm{vec} \mathbf{A}^{\prime}}{d \boldsymbol{\theta}^{\prime \top}}\right)^{\top}\left(\mathbf{w}^{\prime} \otimes \mathbf{v}^{\prime}\right)\right]
$$

To evaluate (3.72), we will use the following rule (Magnus 2010) for derivatives of matrix products. Given matrices $\mathbf{Y}(n \times v)$ and $\mathbf{X}(m \times n)$, the derivative of their product with respect to a third matrix $\mathbf{Z}(p \times q)$ is

$$
\frac{d \operatorname{vec}(\mathbf{X Y})}{d(\operatorname{vec} \mathbf{Z})^{\top}}=\left(\mathbf{Y}^{\boldsymbol{\top}} \otimes \mathbf{I}_{m}\right) \frac{d \operatorname{vec} \mathbf{X}}{d(\operatorname{vec} \mathbf{Z})^{\top}}+\left(\mathbf{I}_{v} \otimes \mathbf{X}\right) \frac{d \operatorname{vec} \mathbf{Y}}{d(\operatorname{vec} \mathbf{Z})^{\top}}
$$

Using (3.73), we can rewrite (3.72) as:

$$
\begin{aligned}
\frac{\partial^{2} \lambda^{\prime}}{\partial \boldsymbol{\theta} \partial \boldsymbol{\theta}^{\prime \top}} & =\left(\mathbf{w}^{\prime \top} \otimes \mathbf{v}^{\prime \top} \otimes \mathbf{I}_{s}\right) \frac{d}{d \boldsymbol{\theta}^{\top}} \operatorname{vec}\left[\left(\frac{d \operatorname{vec} \mathbf{A}^{\prime}}{d \boldsymbol{\theta}^{\prime \top}}\right)^{\top}\right]+\left(\frac{d \operatorname{vec} \mathbf{A}^{\prime}}{d \boldsymbol{\theta}^{\prime \top}}\right)^{\top} \frac{d \operatorname{vec}\left(\mathbf{w}^{\prime} \otimes \mathbf{v}^{\prime}\right)}{d \boldsymbol{\theta}^{\top}} \\
& =\left(\mathbf{w}^{\prime \top} \otimes \mathbf{v}^{\prime \top} \otimes \mathbf{I}_{s}\right) \frac{d \operatorname{vec}\left(\mathbf{C}^{\top}\right)}{d \boldsymbol{\theta}^{\top}}+\mathbf{C}^{\top} \frac{d \operatorname{vec}\left(\mathbf{w}^{\prime} \otimes \mathbf{v}^{\prime}\right)}{d \boldsymbol{\theta}^{\top}}
\end{aligned}
$$

where

$$
\mathbf{C}=\frac{d \operatorname{vec} \mathbf{A}^{\prime}}{d \boldsymbol{\theta}^{\prime \top}}
$$

To evaluate the derivative in the first term of (3.74), recall $\mathbf{A}^{\prime}$ is a matrix function of $\mathbf{w}(\boldsymbol{\theta})$. Thus, to find the matrix derivative of $\mathbf{C}$ with respect to $\boldsymbol{\theta}$, apply the commutation matrix and chain rule for matrix derivatives:

$$
\begin{aligned}
\frac{d \operatorname{vec}\left(\mathbf{C}^{\top}\right)}{d \boldsymbol{\theta}^{\top}} & =\mathbf{K}_{n^{2}, s} \frac{d \operatorname{vec}(\mathbf{C})}{d \boldsymbol{\theta}^{\top}} \\
& =\mathbf{K}_{n^{2}, s} \frac{d \operatorname{vec}(\mathbf{C}[\mathbf{w}(\boldsymbol{\theta})])}{d \boldsymbol{\theta}^{\top}} \\
& =\mathbf{K}_{n^{2}, s} \frac{d \operatorname{vec} \mathbf{C}}{d \mathbf{w}^{\top}} \frac{d \mathbf{w}}{d \boldsymbol{\theta}^{\top}}
\end{aligned}
$$

where it can be shown (Caswell 2008) that

$$
\frac{d \mathbf{w}}{d \boldsymbol{\theta}^{\top}}=\left[\lambda \mathbf{I}_{s}-\mathbf{A}+\mathbf{w} \mathbf{e}^{\top} \mathbf{A}-\left[\mathbf{w}^{\top} \otimes\left(\mathbf{I}_{s}-\mathbf{w}^{\top}\right)\right] \frac{d \operatorname{vec} \mathbf{A}}{d \mathbf{w}^{\top}}\right]^{-1}\left[\mathbf{w}^{\top} \otimes\left(\mathbf{I}_{s}-\mathbf{w} \mathbf{e}^{\top}\right)\right] \frac{d \operatorname{vec} \mathbf{A}}{d \boldsymbol{\theta}^{\top}}
$$


To evaluate the derivative in the second term of (3.74), we will use the following rule (Magnus and Neudecker 1999, p. 227) for the derivatives of Kronecker products. Given matrices $\mathbf{Y}(u \times v)$ and $\mathbf{X}(m \times n)$, the derivative of their Kronecker product with respect to a third matrix $\mathbf{Z}(p \times q)$ is

$$
\frac{d \operatorname{vec}(\mathbf{X} \otimes \mathbf{Y})}{d(\operatorname{vec} \mathbf{Z})^{\top}}=\left(\mathbf{I}_{n} \otimes \mathbf{K}_{v m} \otimes \mathbf{I}_{u}\right)\left[\left(\mathbf{I}_{m n} \otimes \operatorname{vec} \mathbf{Y}\right) \frac{d \operatorname{vec} \mathbf{X}}{d(\operatorname{vec} \mathbf{Z})^{\top}}+\left(\operatorname{vec} \mathbf{X} \otimes \mathbf{I}_{u v}\right) \frac{d \operatorname{vec} \mathbf{Y}}{d(\operatorname{vec} \mathbf{Z})^{\top}}\right]
$$

Using (3.78), the derivative in the second term of (3.74) becomes:

$$
\frac{d \operatorname{vec}\left(\mathbf{w}^{\prime} \otimes \mathbf{v}^{\prime}\right)}{d \boldsymbol{\theta}^{\top}}=\left(\mathbf{I}_{n} \otimes \mathbf{v}^{\prime}\right) \frac{d \mathbf{w}^{\prime}}{d \boldsymbol{\theta}^{\top}}+\left(\mathbf{w}^{\prime} \otimes \mathbf{I}_{n}\right) \frac{d \mathbf{v}^{\prime}}{d \boldsymbol{\theta}^{\top}}
$$

By chain rule,

$$
\frac{d \mathbf{w}^{\prime}}{d \boldsymbol{\theta}^{\top}}=\frac{d \mathbf{w}^{\prime}}{d \operatorname{vec} \mathbf{A}^{\prime \top}} \frac{d \operatorname{vec} \mathbf{A}^{\prime}}{d \mathbf{w}^{\top}} \frac{d \mathbf{w}}{d \boldsymbol{\theta}^{\top}}
$$

where $\frac{d \mathbf{w}^{\prime}}{d v \operatorname{vec} \mathbf{A}^{\prime \top}}$ is given by (3.26). Similarly,

$$
\frac{d \mathbf{v}^{\prime}}{d \boldsymbol{\theta}^{\top}}=\frac{d \mathbf{v}^{\prime}}{d \operatorname{vec} \mathbf{A}^{\prime \top}} \frac{d \operatorname{vec} \mathbf{A}^{\prime}}{d \mathbf{w}^{\top}} \frac{d \mathbf{w}}{d \boldsymbol{\theta}^{\top}}
$$

where $\frac{d \mathbf{v}^{\prime}}{d \operatorname{vec}^{\prime \top}}$ is given by $(3.27)$.

Substituting (3.76) and (3.79) into (3.74), we obtain:

$$
\frac{\partial^{2} \lambda^{\prime}}{\partial \boldsymbol{\theta} \partial \boldsymbol{\theta}^{\prime \top}}=\left(\mathbf{w}^{\prime \top} \otimes \mathbf{v}^{\prime \top} \otimes \mathbf{I}_{s}\right) \mathbf{K}_{n^{2}, s} \frac{d \operatorname{vec} \mathbf{C}}{d \mathbf{w}^{\top}} \frac{d \mathbf{w}}{d \boldsymbol{\theta}^{\top}}+\mathbf{C}^{\top}\left[\left(\mathbf{I}_{n} \otimes \mathbf{v}^{\prime}\right) \frac{d \mathbf{w}^{\prime}}{d \boldsymbol{\theta}^{\top}}+\left(\mathbf{w}^{\prime} \otimes \mathbf{I}_{n}\right) \frac{d \mathbf{v}^{\prime}}{d \boldsymbol{\theta}^{\top}}\right]
$$

This expression is equivalent to (3.32) when the only evolving trait is the primary sex ratio, so that $\boldsymbol{\theta}=s_{1}$.

\section{Bibliography}

[1] Magnus JR. 2010. On the concept of matrix derivative. Journal of Multivariate Analysis. 101: 2200-2206.

[2] Magnus JR, Neudecker H. 1999. Matrix differential calculus with applications in statistics and econometrics. John Wiley and Sons Ltd. 


\section{Chapter 4}

\section{Calculating second derivatives of population growth rates for ecology and evolution $^{1}$}

\subsection{Abstract}

1. Second derivatives of the population growth rate measure the curvature of its response to demographic, physiological, or environmental parameters. The second derivatives quantify the response of sensitivity results to perturbations, provide a classification of types of selection, and provide one way to calculate sensitivities of the stochastic growth rate.

2. Using matrix calculus, we derive the second derivatives of three population growth rate measures: the discrete-time growth rate $\lambda$, the continuous-time growth rate $r=\log \lambda$, and the net reproductive rate $R_{0}$, which measures per-generation growth.

3. We present a suite of formulae for the second derivatives of each growth rate, and show how to compute these derivatives with respect to projection matrix entries and to lower-level parameters affecting those matrix entries.

4. We also illustrate several ecological and evolutionary applications for these second derivative calculations with a case study for the tropical herb Calathea ovandensis.

Keywords: matrix population models, sensitivity analysis, eigenvalues, invasion exponent, net reproductive rate, Hessian matrix

\footnotetext{
${ }^{1}$ Originally published as "Shyu E, Caswell H. 2014. Calculating second derivatives of population growth rates for ecology and evolution. Methods in Ecology and Evolution. 5: 473-482." Reproduced here under the terms of the Creative Commons Attribution License, which permits use, distribution and reproduction in any medium, provided the original work is properly cited.
} 


\begin{tabular}{|c|c|c|}
\hline Second derivative & Sign & Interpretations \\
\hline \multirow{3}{*}{$\frac{\partial^{2} \lambda}{\partial \theta^{2}}$} & $=0$ & $\begin{array}{l}\text { - sensitivity of } \lambda \text { to } \theta \text { is independent of } \theta \\
\text { - linear selection on trait } \theta\end{array}$ \\
\hline & $>0$ & $\begin{array}{l}\text { - sensitivity of } \lambda \text { to } \theta \text { increases with } \theta \\
\text { - convex selection on trait } \theta \\
\text { - evolutionarily-unstable singular strategy }\end{array}$ \\
\hline & $<0$ & $\begin{array}{l}\text { - sensitivity of } \lambda \text { to } \theta \text { decreases with increases in } \theta \\
\text { - concave selection on trait } \theta \\
\text { - evolutionarily-stable singular strategy }\end{array}$ \\
\hline \multirow{2}{*}{$\frac{\partial^{2} \lambda}{\partial \theta_{j} \partial \theta_{i}}$} & $>0$ & $\begin{array}{l}\text { - sensitivity of } \lambda \text { to } \theta_{i} \text { increases with } \theta_{j} \\
\text { - selection to increase correlation between traits } \theta_{j} \text { and } \theta_{i}\end{array}$ \\
\hline & $<0$ & $\begin{array}{l}\text { - sensitivity of } \lambda \text { to } \theta_{i} \text { decreases with increases in } \theta_{j} \\
\text { - selection to decrease correlation between traits } \theta_{j} \text { and } \theta_{i}\end{array}$ \\
\hline$H[\lambda ; \operatorname{vec} \overline{\mathbf{A}}]$ & $\mathrm{N} / \mathrm{A}$ & - used to calculate sensitivity of the stochastic growth rate $\lambda_{s}(4.5)$ \\
\hline
\end{tabular}

Table 4.1: . Potential applications for the pure and mixed second derivatives of $\lambda$. Analogous interpretations apply to $r$ or $R_{0}$ as alternative measures of growth or fitness.

\subsection{Introduction}

Using matrix population models, ecological indices can be calculated as functions of vital rates like survival or fertility. Measures of population growth rate, including the discrete-time growth rate $\lambda$, the continuous-time growth rate $r=\log \lambda$, and the net reproductive rate $R_{0}$ are of particular interest. The discrete-time population growth rate $\lambda$ is given by the dominant eigenvalue of the population projection matrix in linear or frequency-dependent population models. Sensitivities (first partial derivatives) of $\lambda$ with respect to relevant parameters quantify how population growth responds to vital rate perturbations. These first derivatives are used to project the effects of vital rate changes due to environmental or management perturbations, uncertainty in parameter estimates, and phenotypic evolution (i.e., with $\lambda$ as a fitness measure, the sensitivity of $\lambda$ with respect to a parameter is the selection gradient on that parameter) (Caswell 2001).

The second derivatives of growth rates also have applications in both ecology (e.g., assessing and improving recommendations from sensitivity analysis, approximating the sensitivities of stochastic growth rates) and evolution (e.g., characterizing nonlinear selection gradients and evolutionary equilibria). Several of these applications are summarized in Table 4.1 and described in the following section. 


\subsubsection{Applications of second derivatives of growth rates}

\subsubsection{Second-order sensitivity analysis and growth rate estimation}

The sensitivity of growth rate provides insight into the population response to parameter perturbations. However, such perturbations also affect the sensitivity itself, i.e. sensitivity is "situational" (Stearns 1992). These effects are quantified by the sensitivity, with respect to a parameter $\theta_{j}$, of the sensitivity of $\lambda$ to another parameter $\theta_{i}$, i.e. by the second derivatives $\frac{\partial^{2} \lambda}{\partial \theta_{j} \partial \theta_{i}}$. The sensitivity of the elasticity of growth rate to parameters similarly depends on second derivatives (Caswell 1996, 2001).

Second derivatives can be applied to population projections previously based on first-order sensitivity analysis. While managers often focus on the vital rates to which population growth is particularly sensitive or elastic, these first-order results may change depending on parameter perturbations. First derivatives also provide a linear, first-order approximation to the response of the growth rate to changes in parameters. The linear approximation is guaranteed to be accurate for sufficiently small perturbations, and often does very well for quite large perturbations. If the response of $\lambda$ to $\theta$ is nonlinear, it is tempting to use a second-order approximation for $\Delta \lambda$ :

$$
\Delta \lambda \approx \sum_{i} \frac{\partial \lambda}{\partial \theta_{i}} \Delta \theta_{i}+\sum_{i} \frac{1}{2} \frac{\partial^{2} \lambda}{\partial \theta_{i}^{2}}\left(\Delta \theta_{i}\right)^{2}+\sum_{i \neq j} \frac{\partial^{2} \lambda}{\partial \theta_{i} \partial \theta_{j}}\left(\Delta \theta_{i}\right)\left(\Delta \theta_{j}\right)
$$

Although this may, in some cases, provide a more accurate calculation, this is not guaranteed; as shown in Figure 1 of Carslake et al. (2008), adding the second-order terms may actually reduce the accuracy of the approximation.

\subsubsection{Characterizing nonlinear selection processes}

Nonlinear relationships between fitness and trait values have consequences for selection. When fitness is a linear function of a trait, selection affects only the trait's mean value. When fitness is a nonlinear function of the trait, however, selection changes the trait's variance or higher moments. A concave fitness function produces concave selection, which reduces trait variance. A convex fitness function produces convex selection, which increases trait variance (Lande and Arnold 1983, Phillips and Arnold 1989, Brodie et al. 1995).

One can classify a selection process as linear, concave, or convex using quadratic selection gradients, the local second derivatives of fitness with respect to trait value (Phillips and Arnold 1989). If fitness is measured as $\lambda$, these quadratic selection gradients are equivalent to $\partial^{2} \lambda / \partial \theta^{2}$, the pure second derivatives of $\lambda$ with respect to trait $\theta$ (e.g., the second derivatives with respect to stage-specific survival in C. ovandensis, as shown in Figure 4-3a). Concave, linear, and convex selection correspond to negative, zero, and positive second derivatives, respectively.

If $\partial^{2} \lambda / \partial \theta^{2}=0$, the selection process is linear and can only shift the mean of trait $\theta$. If $\partial^{2} \lambda / \partial \theta^{2}>0$, selection is convex and can increase trait variance. If $\partial^{2} \lambda / \partial \theta^{2}<0$, selection is concave 
and can reduce trait variance. When the pure second derivatives of two different traits, $\theta_{i}$ and $\theta_{j}$, are both nonzero, their mixed second derivative $\partial^{2} \lambda / \partial \theta_{j} \partial \theta_{i}$ indicates the effect of correlational selection. Mixed second derivatives $\partial^{2} \lambda / \partial \sigma_{1} \partial \sigma_{i}$ and $\partial^{2} \lambda / \partial \sigma_{2} \partial \sigma_{i}$ for C. ovandensis, for example, are shown in Figures 4-3b and 4-3c. If $\partial^{2} \lambda / \partial \theta_{j} \partial \theta_{i}<0$, there is selection to decrease the phenotypic correlation between the two traits; if $\partial^{2} \lambda / \partial \theta_{j} \partial \theta_{i}>0$, there is selection to increase their correlation.

\subsubsection{Stability of evolutionary singular strategies}

Evolutionary singular strategies (SSs) are phenotypes for which the selection gradient is locally zero (e.g., Geritz et al. 1998). SSs are classified as stable, attracting, or repelling, and by whether they can invade or coexist with other nearby phenotypes (Geritz et al. 1998, Diekmann 2004, Waxman and Gavrilets 2005, Doebeli 2011).

These classifications depend on the local second derivatives of invasion fitness. Invasion fitness is the growth rate of a rare mutant in an equilibrium resident environment. For example, the second derivative of the mutant growth rate $\lambda$ to the mutant trait $y$ determines whether a SS is evolutionarily-stable $\left(\partial^{2} \lambda / \partial y^{2}<0\right)$ or evolutionarily-unstable $\left(\partial^{2} \lambda / \partial y^{2}>0\right)$. Evolutionarily-stable strategies, once established, are unbeatable phenotypes against which no nearby mutants can increase under selection, and are thus long-term evolutionary endpoints. Evolutionarily-unstable strategies, on the other hand, are branching points open to phenotypic divergence and may ultimately become sources of sympatric speciation (Geritz et al. 1998).

\subsubsection{Sensitivity of the stochastic growth rate}

In a stochastic environment, population growth is measured by the stochastic growth rate

$$
\log \lambda_{s}=\lim _{t \rightarrow \infty} \frac{1}{t} \log N(t)
$$

where $N(t)$ is the population size at time $t$.

Tuljapurkar (1982) derived an approximation for the stochastic growth rate when variability around the mean vital rates is small and temporally independent. As shown by Caswell (2001, Section 14.3.6), this approximation can be written in terms of the first derivatives of $\lambda$, the dominant eigenvalue of the mean projection matrix $\overline{\mathbf{A}}$. Letting $\mathbf{D}=D[\lambda ; \operatorname{vec} \overline{\mathbf{A}}]$ be the Jacobian matrix of first derivatives,

$$
\log \lambda_{s} \approx \log \lambda-\frac{\mathbf{D C D}^{\mathbf{T}}}{2 \lambda^{2}}
$$

where $\mathbf{C}$ is the covariance matrix of the entries of the population projection matrix

$$
\mathbf{C}=E\left[\left(\operatorname{vec} \mathbf{A}_{t}-\operatorname{vec} \overline{\mathbf{A}}\right)\left(\operatorname{vec} \mathbf{A}_{t}-\operatorname{vec} \overline{\mathbf{A}}\right)^{\top}\right]
$$

A measure of the sensitivity of the stochastic growth rate can be obtained by differentiating (4.3) with respect to the entries of $\overline{\mathbf{A}}$. The sensitivity of the stochastic growth rate to $\overline{\mathbf{A}}$, leaving 
the variances and covariances fixed, depends on the second derivatives of $\lambda$ as

$$
\frac{d \log \lambda_{s}}{d \operatorname{vec}^{\top} \overline{\mathbf{A}}}=\frac{\mathbf{D}}{\lambda}\left(1-\frac{\mathbf{C H}}{\lambda}+\frac{\mathbf{D C D}^{\boldsymbol{\top}}}{\lambda^{2}}\right)
$$

where $\mathbf{H}=H[\lambda ; \operatorname{vec} \overline{\mathbf{A}}]$ is the Hessian matrix of second derivatives. A derivation of (4.5) is provided in Appendix 4.D.

\subsubsection{Calculating second derivatives of growth rates}

The second derivatives of $\lambda$ with respect to matrix elements were introduced by Caswell (1996; see also Caswell 2001, Section 9.7). However, the calculations in Caswell (1996) are awkward and error-prone, because they involve all the eigenvalues and eigenvectors of the projection matrix. McCarthy et al. (2008) introduced an alternative approach for calculating the second derivatives of eigenvalues (they call them "second-order sensitivities") based on transfer functions, partially to avoid the calculation of all the eigenvectors. However, they consider only rank-one perturbations of a subset of the matrix elements, excluding fertilities.

Here, we reformulate the second derivative calculations using matrix calculus, providing easily computable results. We extend previous results by including not only second derivatives with respect to matrix elements, but also those with respect to any lower-level parameters that may affect the matrix elements. We also present not only second derivatives of $\lambda$, but also those of the continuous-time invasion exponent $r$ and the net reproductive rate $R_{0}$.

The key to our approach is that the calculation of first derivatives using matrix calculus yields a particular expression, the differentiation of which leads directly to the second derivatives. Second derivatives are easily computed by this method in any matrix-oriented language, such as MATLAB or R. Although we consider only the second derivatives of population growth rates, our approach extends naturally to other scalar dependent variables.

We will illustrate the ecological and evolutionary applications of these second derivative calculations with a case study for the tropical herb Calathea ovandensis.

\subsubsection{Notation}

Matrices are denoted by uppercase boldface letters (e.g., A) and vectors by lowercase boldface letters (e.g., w); unless otherwise indicated, all vectors are column vectors. Transposes of matrices and vectors are indicated by the superscript ${ }^{\top}$. The matrix $\mathbf{I}_{n}$ is the $n \times n$ identity matrix, the vector $\mathbf{e}$ is a vector of ones, and $\mathbf{e}_{1}$ is a vector with 1 as its first entry and zeros elsewhere. The matrix $\mathbf{K}_{m, n}$ is a $m n \times m n$ commutation matrix (vec-permutation matrix) (Magnus and Neudecker 1979, Henderson and Searle 1981), which can be calculated using the MATLAB function provided in Appendix 4.E. The expression $\operatorname{diag}(\mathbf{x})$ indicates the square matrix with $\mathbf{x}$ on the diagonal and zeros elsewhere. 
The Kronecker product is denoted by $\mathbf{X} \otimes \mathbf{Y}$ and the Hadamard (element-by-element) product by $\mathbf{X} \circ \mathbf{Y}$. The vec operator (e.g., vec $\mathbf{A}$ ) stacks the columns of a matrix into a single vector. For convenience, we will write $(\operatorname{vec} \mathbf{A})^{\top}=\operatorname{vec}^{\top} \mathbf{A}$. We will make frequent use of Roth's theorem (Roth 1934), which states that for any matrices $\mathbf{X}, \mathbf{Y}$, and Z:

$$
\operatorname{vec}(\mathbf{X Y Z})=\left(\mathbf{Z}^{\boldsymbol{\top}} \otimes \mathbf{X}\right) \operatorname{vec} \mathbf{Y}
$$

\subsection{Matrix calculus}

\subsubsection{Matrix calculus notation}

Matrix calculus is a system for manipulating vectors and matrices in multivariable calculus, and simplifies partial derivative calculations by allowing the differentiation of scalar, vector, or matrix functions with respect to scalar, vector, or matrix arguments. While there are multiple matrix calculus notations, we will use the system of Magnus and Neudecker (1999). For a more detailed introduction to these methods in an ecological context, see Appendix 1 of Caswell (2007).

The first derivative of a $m \times 1$ vector $\mathbf{y}$ with respect to a $n \times 1$ vector $\mathbf{x}$ is defined to be the $m \times n$ Jacobian matrix

$$
\frac{d \mathbf{y}}{d \mathbf{x}^{\top}}=\left(\frac{d y_{i}}{d x_{j}}\right)
$$

that is, a matrix whose $(i, j)$ entry is the derivative of $y_{i}$ with respect to $x_{j}$. We will also write this as an operator $D[\mathbf{y} ; \mathbf{x}]$; the first argument of $D$ is the vector-valued function $\mathbf{y}$ to be differentiated, and the second argument is the vector-valued variable $\mathbf{x}$ with respect to which differentation is carried out. Thus,

$$
D[\mathbf{y} ; \mathbf{x}]=\frac{d \mathbf{y}}{d \mathbf{x}^{\top}}
$$

As in the scalar case, second derivatives are obtained by differentiating first derivatives. If we consider a scalar-valued function $y(\mathbf{x})$ of a vector-valued argument $\mathbf{x}$, the matrix of second derivatives (the Hessian matrix) is given by the operator

$$
\begin{aligned}
H[y ; \mathbf{x}] & =\frac{d^{2} y}{d \mathbf{x} d \mathbf{x}^{\top}} \\
& =\left(\frac{d^{2} y}{d x_{i} d x_{j}}\right) \\
& =\frac{d}{d \mathbf{x}^{\top}}\left[\left(\frac{d y}{d \mathbf{x}^{\top}}\right)^{\top}\right]
\end{aligned}
$$


The matrix of second derivatives of a vector-valued function $\mathbf{y}(\mathbf{x})$, where $\mathbf{y}$ has dimensions $m \times 1$, is obtained by stacking the Hessian matrices for each of the elements of $\mathbf{y}$; that is,

$$
\begin{aligned}
H[\mathbf{y} ; \mathbf{x}] & =\left(\begin{array}{c}
H\left[y_{1} ; \mathbf{x}\right] \\
H\left[y_{2} ; \mathbf{x}\right] \\
\vdots \\
H\left[y_{m} ; \mathbf{x}\right]
\end{array}\right) \\
& =\frac{d}{d \mathbf{x}^{\top}} \operatorname{vec}\left[\left(\frac{d \mathbf{y}}{d \mathbf{x}^{\top}}\right)^{\top}\right] .
\end{aligned}
$$

These first and second derivative definitions are written in terms of vector-valued functions and arguments. When matrices appear, they are transformed into vectors using the vec operator, which stacks the columns of the matrix into a column vector. Thus, the first and second derivatives of $\lambda$ with respect to the entries of the matrix $\mathbf{A}$ would be written, respectively, as $D[\lambda ; \operatorname{vec} \mathbf{A}]$ and $H[\lambda ; \operatorname{vec} \mathbf{A}]$.

\subsubsection{The identification theorems}

Magnus and Neudecker $(1985,1999)$ showed how to obtain first and second derivatives from the differentials of functions. Their "first identification theorem" showed that

$$
d \mathbf{y}=\mathbf{Q} d \mathbf{x} \quad \Longrightarrow \quad D[\mathbf{y} ; \mathbf{x}]=\mathbf{Q}
$$

That is, if an expression of the form $d \mathbf{y}=\mathbf{Q} d \mathbf{x}$ can be obtained, then the Jacobian matrix of first derivatives is given by $\mathbf{Q}$.

The "second identification theorem" does the same for the Hessian matrix of second derivatives, showing that

$$
d^{2} y=d \mathbf{x}^{\top} \mathbf{B} d \mathbf{x} \quad \Longrightarrow \quad H[y ; \mathbf{x}]=\frac{1}{2}\left(\mathbf{B}+\mathbf{B}^{\top}\right) .
$$

Thus, our goal will be to find expressions of the form $d^{2} y=d \mathbf{x}^{\top} \mathbf{B} d \mathbf{x}$, where $y$ is a measure of population growth rate and $\mathbf{x}$ represents either matrix entries or lower-level parameters; the matrix B will then provide the Hessian matrix using (4.15). The key to our approach is to begin with the expression (4.14) for the first differential, differentiate it to obtain the second differential, and manipulate the results to obtain a matrix $\mathbf{B}$ in the form of (4.15).

\subsection{Second derivatives of growth rates}

We now apply the identification theorems to three measures of population growth rate, the discrete-time growth rate $\lambda$, the continuous-time growth rate $r=\log \lambda$, and the net reproductive rate $R_{0}$. 


\subsubsection{Second derivatives of the discrete-time growth rate $\lambda$}

\subsubsection{Second derivatives of $\lambda$ with respect to matrix entries: $H[\lambda ; \operatorname{vec} \mathbf{A}]$}

We assume a population projection matrix $\mathbf{A}$ of dimension $n \times n$. The discrete-time growth rate $\lambda$ is the dominant eigenvalue of $\mathbf{A}$. To derive $H[\lambda$; vec $\mathbf{A}]$, we begin with an expression of the form (4.14) for the first differential of $\lambda$. As shown in Caswell (2010),

$$
d \lambda=\left(\mathbf{w}^{\top} \otimes \mathbf{v}^{\top}\right) d \operatorname{vec} \mathbf{A}
$$

where $\mathbf{w}$ and $\mathbf{v}$ are the right and left eigenvectors of $\mathbf{A}$ corresponding to $\lambda$, scaled so that

$$
\begin{aligned}
& \mathbf{v}^{\top} \mathbf{w}=1 \\
& \mathbf{e}^{\top} \mathbf{w}=1
\end{aligned}
$$

where $\mathbf{e}$ is a $n \times 1$ vector of ones (see Appendix 4.A).

Differentiate (4.16) to obtain the second differential

$$
d^{2} \lambda=d\left(\mathbf{w}^{\boldsymbol{\top}} \otimes \mathbf{v}^{\boldsymbol{\top}}\right) d \operatorname{vec} \mathbf{A}+\left(\mathbf{w}^{\boldsymbol{\top}} \otimes \mathbf{v}^{\boldsymbol{\top}}\right) d^{2} \operatorname{vec} \mathbf{A}
$$

Because we are calculating second derivatives with respect to $\mathbf{A}$, the second term will drop out because $d^{2} \operatorname{vec} \mathbf{A}=0$ (Magnus 2007). Apply the vec operator to obtain

$$
d^{2} \lambda=d\left(\operatorname{vec}^{\top} \mathbf{A}\right) d \operatorname{vec}\left(\mathbf{w}^{\top} \otimes \mathbf{v}^{\top}\right)
$$

The differential of vec $\left(\mathbf{w}^{\top} \otimes \mathbf{v}^{\top}\right)$ is

$$
d \operatorname{vec}\left(\mathbf{w}^{\boldsymbol{\top}} \otimes \mathbf{v}^{\boldsymbol{\top}}\right)=\left(\mathbf{I}_{n} \otimes \mathbf{v}\right) d \mathbf{w}+\left(\mathbf{w} \otimes \mathbf{I}_{n}\right) d \mathbf{v}
$$

(Magnus and Neudecker 1999). Substituting (4.21) into (4.20) gives

$$
d^{2} \lambda=d\left(\operatorname{vec}^{\top} \mathbf{A}\right)\left[\left(\mathbf{I}_{n} \otimes \mathbf{v}\right) d \mathbf{w}+\left(\mathbf{w} \otimes \mathbf{I}_{n}\right) d \mathbf{v}\right]
$$

By the chain rule,

$$
\begin{aligned}
& d \mathbf{w}=\frac{d \mathbf{w}}{d \operatorname{vec}^{\top} \mathbf{A}} d \operatorname{vec} \mathbf{A} \\
& d \mathbf{v}=\frac{d \mathbf{v}}{d \operatorname{vec}^{\top} \mathbf{A}} d \operatorname{vec} \mathbf{A}
\end{aligned}
$$

The first derivatives of $\mathbf{w}$ and $\mathbf{v}$, subject to (4.17) and (4.18), are given in Caswell (2008) and Caswell and Vindenes (in prep), respectively, as: 


$$
\begin{aligned}
& \frac{d \mathbf{w}}{d \operatorname{vec}^{\top} \mathbf{A}}=\left(\lambda \mathbf{I}_{n}-\mathbf{A}+\mathbf{w} \mathbf{e}^{\top} \mathbf{A}\right)^{-1}\left[\mathbf{w}^{\top} \otimes\left(\mathbf{I}_{n}-\mathbf{w} \mathbf{e}^{\boldsymbol{\top}}\right)\right] \\
& \frac{d \mathbf{v}}{d \operatorname{vec}^{\top} \mathbf{A}}=\left(\lambda \mathbf{I}_{n}-\mathbf{A}^{\top}+\lambda \mathbf{v} \mathbf{w}^{\boldsymbol{\top}}\right)^{-1}\left(\left[\left(\mathbf{I}_{n}-\mathbf{v} \mathbf{w}^{\top}\right) \otimes \mathbf{v}^{\top}\right]-\lambda\left(\mathbf{v} \otimes \mathbf{v}^{\top}\right) \frac{d \mathbf{w}}{d \operatorname{vec}^{\top} \mathbf{A}}\right) .
\end{aligned}
$$

Derivations of (4.25) and (4.26) are provided in Appendix 4.A.

Rewrite (4.22) as

$$
d^{2} \lambda=d \operatorname{vec}^{\top} \mathbf{A}\left[\left(\mathbf{I}_{n} \otimes \mathbf{v}\right) \frac{d \mathbf{w}}{d \operatorname{vec}^{\top} \mathbf{A}}+\left(\mathbf{w} \otimes \mathbf{I}_{n}\right) \frac{d \mathbf{v}}{d \operatorname{vec}^{\top} \mathbf{A}}\right] d \operatorname{vec} \mathbf{A}
$$

This is of the form

$$
d^{2} \lambda=\left(d \operatorname{vec}^{\top} \mathbf{A}\right) \mathbf{B}(d \operatorname{vec} \mathbf{A})
$$

and hence

$$
H[\lambda ; \operatorname{vec} \mathbf{A}]=\frac{1}{2}\left(\mathbf{B}+\mathbf{B}^{\top}\right)
$$

where

$$
\mathbf{B}=\left(\mathbf{I}_{n} \otimes \mathbf{v}\right) \frac{d \mathbf{w}}{d \operatorname{vec}^{\top} \mathbf{A}}+\left(\mathbf{w} \otimes \mathbf{I}_{n}\right) \frac{d \mathbf{v}}{d \operatorname{vec}^{\top} \mathbf{A}}
$$

and the first derivatives of $\mathbf{w}$ and $\mathbf{v}$ are given by (4.25) and (4.26).

\subsubsection{Second derivatives of $\lambda$ with respect to lower-level parameters: $H[\lambda ; \boldsymbol{\theta}]$}

Because many life history traits and environmental factors affect multiple life cycle transitions, the entries of $\mathbf{A}$ are usually functions of lower-level parameters. To calculate the second derivatives of $\lambda$ with respect to a $s \times 1$ parameter vector $\boldsymbol{\theta}$, we must develop a Hessian chain rule.

Again, we begin with the first differential of $\lambda$ in (4.16), and differentiate to obtain the second differential (4.19). Because we are calculating second derivatives with respect to $\boldsymbol{\theta}$ rather than $\mathbf{A}$, $d^{2}$ vec $\mathbf{A}$ is no longer zero. By the chain rule,

$$
d \operatorname{vec} \mathbf{A}=\frac{d \mathrm{vec} \mathbf{A}}{d \boldsymbol{\theta}^{\top}} d \boldsymbol{\theta} .
$$

Differentiate (4.30) to obtain

$$
d^{2} \operatorname{vec} \mathbf{A}=d\left(\frac{d \operatorname{vec} \mathbf{A}}{d \boldsymbol{\theta}^{\top}}\right) d \boldsymbol{\theta}+\frac{d \operatorname{vec} \mathbf{A}}{d \boldsymbol{\theta}^{\top}} d^{2} \boldsymbol{\theta}
$$

Because $d^{2} \boldsymbol{\theta}=0$, the second term drops out. 
Substituting (4.30) and (4.31) into the expression for the second differential in (4.19) yields

$$
d^{2} \lambda=d\left(\mathbf{w}^{\boldsymbol{\top}} \otimes \mathbf{v}^{\top}\right) \frac{d \operatorname{vec} \mathbf{A}}{d \boldsymbol{\theta}^{\top}} d \boldsymbol{\theta}+\left(\mathbf{w}^{\boldsymbol{\top}} \otimes \mathbf{v}^{\top}\right) d\left(\frac{d \operatorname{vec} \mathbf{A}}{d \boldsymbol{\theta}^{\top}}\right) d \boldsymbol{\theta}
$$

To simplify this expression, define

$$
\begin{aligned}
\mathbf{S} & =\frac{d \operatorname{vec} \mathbf{A}}{d \boldsymbol{\theta}^{\top}} \\
\mathbf{T} & =\frac{d \lambda}{d \operatorname{vec}^{\top} \mathbf{A}}=\mathbf{w}^{\top} \otimes \mathbf{v}^{\top}
\end{aligned}
$$

in terms of which (4.32) can be rewritten as

$$
d^{2} \lambda=[(d \mathbf{T}) \mathbf{S}+\mathbf{T}(d \mathbf{S})] d \boldsymbol{\theta}
$$

Then apply the vec operator and Roth's theorem (4.6) to obtain

$$
\begin{aligned}
d^{2} \lambda & =d \boldsymbol{\theta}^{\boldsymbol{\top}} \operatorname{vec}[\mathbf{T}(d \mathbf{S})+(d \mathbf{T}) \mathbf{S}] \\
& =d \boldsymbol{\theta}^{\boldsymbol{\top}}\left[\left(\mathbf{I}_{s} \otimes \mathbf{T}\right) d \operatorname{vec} \mathbf{S}+\mathbf{S}^{\boldsymbol{\top}} d \operatorname{vec} \mathbf{T}\right] \\
& =d \boldsymbol{\theta}^{\boldsymbol{\top}}\left[\left(\mathbf{I}_{s} \otimes \mathbf{T}\right) \frac{d \operatorname{vec} \mathbf{S}}{d \boldsymbol{\theta}^{\boldsymbol{\top}}}+\mathbf{S}^{\boldsymbol{\top}} \frac{d \operatorname{vec} \mathbf{T}}{d \boldsymbol{\theta}^{\boldsymbol{\top}}}\right] d \boldsymbol{\theta}
\end{aligned}
$$

where, as shown by (4.86) and (4.89) in Appendix 4.B,

$$
\begin{aligned}
\left(\mathbf{I}_{s} \otimes \mathbf{T}\right) \frac{d \operatorname{vec} \mathbf{S}}{d \boldsymbol{\theta}^{\top}} & =\left(\mathbf{T} \otimes \mathbf{I}_{s}\right) H[\operatorname{vec} \mathbf{A} ; \boldsymbol{\theta}] \\
\frac{d \operatorname{vec} \mathbf{T}}{d \boldsymbol{\theta}^{\top}} & =H[\lambda ; \operatorname{vec} \mathbf{A}] \mathbf{S} .
\end{aligned}
$$

The expression (4.36) is of the form

$$
d^{2} \lambda=d \boldsymbol{\theta}^{\top} \mathbf{B} d \boldsymbol{\theta}
$$

and hence by the second identification theorem (4.15),

$$
H[\lambda ; \boldsymbol{\theta}]=\frac{1}{2}\left(\mathbf{B}+\mathbf{B}^{\boldsymbol{\top}}\right)
$$

where 


$$
\begin{aligned}
\mathbf{B} & =\left(\mathbf{T} \otimes \mathbf{I}_{s}\right) H[\operatorname{vec} \mathbf{A} ; \boldsymbol{\theta}]+\left(\mathbf{S}^{\boldsymbol{\top}}\right) H[\lambda ; \operatorname{vec} \mathbf{A}] \mathbf{S} \\
& =\left(\mathbf{w}^{\boldsymbol{\top}} \otimes \mathbf{v}^{\boldsymbol{\top}} \otimes \mathbf{I}_{s}\right) H[\operatorname{vec} \mathbf{A} ; \boldsymbol{\theta}]+\left(\frac{d \operatorname{vec} \mathbf{A}}{d \boldsymbol{\theta}^{\top}}\right)^{\top} H[\lambda ; \operatorname{vec} \mathbf{A}] \frac{d \operatorname{vec} \mathbf{A}}{d \boldsymbol{\theta}^{\boldsymbol{\top}}}
\end{aligned}
$$

The first and second derivatives of $\mathbf{A}$ with respect to $\boldsymbol{\theta}$, which appear in $\frac{d \mathrm{vec} \mathbf{A}}{d \boldsymbol{\theta}^{\top}}$ and $H[\operatorname{vec} \mathbf{A} ; \boldsymbol{\theta}$ ] respectively, can be evaluated by hand or by using a symbolic math program. This result is in agreement with the Hessian chain rule derived in a different way by Magnus and Neudecker (1999, p. 125).

\subsubsection{Second derivatives of the invasion exponent $r$ : $H[r ; \operatorname{vec} \mathbf{A}]$ and $H[r ; \boldsymbol{\theta}]$}

The population growth rate in continuous time is the invasion exponent $r=\log \lambda$. By the definition of the Hessian in (4.11), the Hessian of $r$ with respect to $\mathbf{A}$ is

$$
H[r ; \operatorname{vec} \mathbf{A}]=\frac{d}{d \operatorname{vec}^{\top} \mathbf{A}} \operatorname{vec}\left[\left(\frac{d \log \lambda}{d \operatorname{vec}^{\top} \mathbf{A}}\right)^{\top}\right] .
$$

We calculate the first derivative of $\log \lambda$,

$$
H[r ; \operatorname{vec} \mathbf{A}]=\frac{d}{d \operatorname{vec}^{\top} \mathbf{A}}\left[\frac{1}{\lambda} \operatorname{vec}\left(\frac{d \lambda}{d \operatorname{vec}^{\top} \mathbf{A}}\right)^{\top}\right]
$$

and then apply the product rule to obtain

$$
H[r ; \operatorname{vec} \mathbf{A}]=\left(\frac{d \lambda}{d \operatorname{vec}^{\top} \mathbf{A}}\right)^{\top} \frac{d}{d \operatorname{vec}^{\top} \mathbf{A}} \frac{1}{\lambda}+\frac{1}{\lambda} \frac{d}{d \operatorname{vec}^{\top} \mathbf{A}} \operatorname{vec}\left[\left(\frac{d \lambda}{\partial \operatorname{vec}^{\top} \mathbf{A}}\right)^{\top}\right]
$$

which simplifies to

$$
\begin{aligned}
H[r ; \operatorname{vec} \mathbf{A}] & =-\frac{1}{\lambda^{2}}\left(\frac{d \lambda}{\partial \operatorname{vec}^{\top} \mathbf{A}}\right)^{\top} \frac{d \lambda}{\partial \operatorname{vec}^{\top} \mathbf{A}}+\frac{1}{\lambda} H[\lambda ; \operatorname{vec} \mathbf{A}] \\
& =-\frac{1}{\lambda^{2}}\left(\mathbf{w} \mathbf{w}^{\top} \otimes \mathbf{v v}^{\top}\right)+\frac{1}{\lambda} H[\lambda ; \operatorname{vec} \mathbf{A}]
\end{aligned}
$$

where $H[\lambda ; \operatorname{vec} \mathbf{A}]$ is given by (4.29).

Replacing vec $\mathbf{A}$ in (4.44) with a parameter vector $\boldsymbol{\theta}$ gives the Hessian 


$$
\begin{aligned}
H[r ; \boldsymbol{\theta}] & =-\frac{1}{\lambda^{2}}\left(\frac{d \lambda}{d \boldsymbol{\theta}^{\top}}\right)^{\top} \frac{d \lambda}{d \boldsymbol{\theta}^{\top}}+\frac{1}{\lambda} H[\lambda ; \boldsymbol{\theta}] \\
& =-\frac{1}{\lambda^{2}}\left(\frac{d \mathrm{vec} \mathbf{A}}{d \boldsymbol{\theta}^{\top}}\right)^{\top}\left(\mathbf{w} \mathbf{w}^{\top} \otimes \mathbf{v} \mathbf{v}^{\top}\right) \frac{d \operatorname{vec} \mathbf{A}}{d \boldsymbol{\theta}^{\top}}+\frac{1}{\lambda} H[\lambda ; \boldsymbol{\theta}]
\end{aligned}
$$

where $\frac{d \mathrm{vec} \mathbf{A}}{\partial \boldsymbol{\theta}^{\mathrm{T}}}$ can be calculated by hand or with a symbolic math program, and $H[\lambda ; \boldsymbol{\theta}]$ can be obtained from (4.40).

\subsubsection{Second derivatives of the net reproductive rate $R_{0}$}

The net reproductive rate $R_{0}$ measures the population growth rate per generation and is used as an alternative fitness measure to $r$ under some special conditions (Pásztor et al. 1996, Brommer 2000). If $\mathbf{A}$ is decomposed into transition and fertility matrices, $\mathbf{A}=\mathbf{U}+\mathbf{F}$, then $R_{0}$ is the dominant eigenvalue of the next generation matrix $\mathbf{R}=\mathbf{F N}$ (Cushing and Zhou 1994), where $\mathbf{N}$ is the fundamental matrix:

$$
\mathbf{N}=\left(\mathbf{I}_{n}-\mathbf{U}\right)^{-1}
$$

The $(i, j)$ entry of $\mathbf{N}$ gives the expected number of visits to stage $i$ for an individual starting in stage $j$. The $(i, j)$ entry of $\mathbf{R}$ gives the expected lifetime production of stage $i$ offspring by an individual starting in stage $j$.

Because $R_{0}$ is an eigenvalue, our results for $H[\lambda$; vec $\mathbf{A}]$ and $H[\lambda ; \boldsymbol{\theta}]$ can be applied to find its second derivatives, but with $\mathbf{R}$ taking the place of matrix $\mathbf{A}$. The resulting expressions are more complicated than the corresponding expressions for $\lambda$, because parameters can affect $R_{0}$ through $\mathbf{U}, \mathbf{F}$, or both. In the important special case where only a single type of offspring is produced (suppose it is numbered as stage 1 ), then $\mathbf{R}$ is a diagonal matrix and $R_{0}$ is its $(1,1)$ entry; in this case, eigenvalue calculations are not necessary.

We defer the fully general calculation of $H\left[R_{0} ; \boldsymbol{\theta}\right]$ to Appendix $\mathrm{C}$, and show results here for the useful special cases $H\left[R_{0} ; \operatorname{vec} \mathbf{U}\right]$ and $H\left[R_{0} ; \operatorname{vec} \mathbf{F}\right]$, for single and multiple types of offspring.

If we apply equations (4.40) to the case of $R_{0}$, we obtain

$$
H\left[R_{0} ; \boldsymbol{\theta}\right]=\frac{1}{2}\left(\mathbf{B}+\mathbf{B}^{\boldsymbol{\top}}\right)
$$

where

$$
\mathbf{B}=\left(\mathbf{w}_{R}^{\top} \otimes \mathbf{v}_{R}^{\top} \otimes \mathbf{I}_{s}\right) H[\operatorname{vec} \mathbf{R} ; \boldsymbol{\theta}]+\left(\frac{d \operatorname{vec} \mathbf{R}}{d \boldsymbol{\theta}^{\top}}\right)^{\top} H\left[R_{0} ; \operatorname{vec} \mathbf{R}\right] \frac{d \operatorname{vec} \mathbf{R}}{d \boldsymbol{\theta}^{\top}}
$$

and $\mathbf{w}_{R}$ and $\mathbf{v}_{R}$ are the right and left eigenvectors of $\mathbf{R}$.

To evaluate (4.48), we must calculate the second derivatives of $R_{0}$ with respect to $\mathbf{R}$, and the first and second derivatives of $\mathbf{R}$ with respect to $\boldsymbol{\theta}$. For the former, the Hessian $H\left[R_{0}\right.$; vec $\left.\mathbf{R}\right]$ is given by (4.29), using the dominant eigenvalues and eigenvectors of $\mathbf{R}$ rather than those of $\mathbf{A}$. For 
the latter, we will consider the derivatives of $\mathbf{R}$ with respect to $\mathbf{U}$ and $\mathbf{F}$ in turn. The derivatives of $\mathbf{R}$ with respect to general parameters $\boldsymbol{\theta}$ are shown in Appendix 4.C.

\subsubsection{Second derivatives of $R_{0}$ to the transition matrix: $H\left[R_{0}\right.$; vec $\left.\mathrm{U}\right]$}

The second derivatives of $R_{0}$ with respect to the entries of the transition matrix $\mathbf{U}$ require the first

and second derivatives of $\mathbf{R}$ with respect to $\mathbf{U}$. The first derivatives are obtained by differentiating $\mathbf{R}=\mathbf{F N}$, applying the vec operator, and noting that $d \operatorname{vec} \mathbf{N}=\left(\mathbf{N}^{\boldsymbol{\top}} \otimes \mathbf{N}\right) d \operatorname{vec} \mathbf{U}$ (Caswell 2006, 2009), to obtain

$$
\frac{d \operatorname{vec} \mathbf{R}}{d \operatorname{vec}^{\top} \mathbf{U}}=\left(\mathbf{N}^{\top} \otimes \mathbf{R}\right)
$$

The second derivatives of $\mathbf{R}$ are obtained from the definition of the Hessian matrix (4.13):

$$
\begin{aligned}
H[\operatorname{vec} \mathbf{R} ; \operatorname{vec} \mathbf{U}] & =\frac{d}{d \operatorname{vec}^{\top} \mathbf{U}^{-}} \operatorname{vec}\left[\left(\frac{d \operatorname{vec} \mathbf{R}}{d \operatorname{vec} \top \mathbf{U}}\right)^{\top}\right] \\
& =\frac{d}{d \operatorname{vec}^{\top} \mathbf{U}} \operatorname{vec}\left[\mathbf{N} \otimes \mathbf{R}^{\top}\right] .
\end{aligned}
$$

The derivative of vec $\left(\mathbf{N} \otimes \mathbf{R}^{\mathbf{T}}\right)$ is given by a result of Magnus and Neudecker (1985, Theorem 11; 1999, p. 209); for a $m \times n$ matrix $\mathbf{X}$ and a $p \times q$ matrix $\mathbf{Y}$,

$$
d \operatorname{vec}(\mathbf{X} \otimes \mathbf{Y})=\left(\mathbf{I}_{n} \otimes \mathbf{K}_{q m} \otimes \mathbf{I}_{p}\right)\left[\left(\mathbf{I}_{m n} \otimes \operatorname{vec} \mathbf{Y}\right) d \operatorname{vec} \mathbf{X}+\left(\operatorname{vec} \mathbf{X} \otimes \mathbf{I}_{p q}\right) d \operatorname{vec} \mathbf{Y}\right]
$$

Thus, (4.50) can be rewritten as

$$
\begin{aligned}
H[\operatorname{vec} \mathbf{R} ; \operatorname{vec} \mathbf{U}] & =\left(\mathbf{I}_{n} \otimes \mathbf{K}_{n, n} \otimes \mathbf{I}_{n}\right)\left[\left(\mathbf{I}_{n^{2}} \otimes \operatorname{vec}\left[\mathbf{R}^{\top}\right]\right) \frac{d \operatorname{vec} \mathbf{N}}{d \operatorname{vec} \mathbf{U}}+\left(\operatorname{vec} \mathbf{N} \otimes \mathbf{I}_{n^{2}}\right) \frac{d \operatorname{vec} \mathbf{R}^{\top}}{d \operatorname{vec} \boldsymbol{\top} \mathbf{U}}\right] \\
& =\left(\mathbf{I}_{n} \otimes \mathbf{K}_{n, n} \otimes \mathbf{I}_{n}\right)\left[\left(\mathbf{I}_{n^{2}} \otimes \operatorname{vec}\left[\mathbf{R}^{\top}\right]\right)\left(\mathbf{N}^{\top} \otimes \mathbf{N}\right)+\left(\operatorname{vec} \mathbf{N} \otimes \mathbf{I}_{n^{2}}\right) \mathbf{K}_{n, n}\left(\mathbf{N}^{\top} \otimes \mathbf{R}\right)\right]
\end{aligned}
$$

As a result,

$$
H\left[R_{0} ; \operatorname{vec} \mathbf{U}\right]=\frac{1}{2}\left(\mathbf{B}+\mathbf{B}^{\top}\right)
$$

where

$$
\mathbf{B}=\left(\mathbf{w}_{R}^{\top} \otimes \mathbf{v}_{R}^{\top} \otimes \mathbf{I}_{n^{2}}\right) H[\operatorname{vec} \mathbf{R} ; \operatorname{vec} \mathbf{U}]+\left(\frac{d \operatorname{vec} \mathbf{R}}{\operatorname{vec}^{\top} \mathbf{U}}\right)^{\top} H\left[R_{0} ; \operatorname{vec} \mathbf{R}\right] \frac{d \operatorname{vec} \mathbf{R}}{\operatorname{vec}^{\top} \mathbf{U}}
$$

where $\frac{d \operatorname{vec} \mathbf{R}}{d \operatorname{vec}^{\top} \mathbf{U}}$ is given by (4.49) and $H[\operatorname{vec} \mathbf{R} ; \operatorname{vec} \mathbf{U}]$ is given by (4.53). 


\subsubsection{Second derivatives of $R_{0}$ to the fertility matrix: $H\left[R_{0} ; \operatorname{vecF}\right]$}

Now consider the second derivatives of $R_{0}$ with respect to the entries of the fertility matrix $\mathbf{F}$. Differentiating $\mathbf{R}=\mathbf{F N}$ with respect to $\mathbf{F}$ yields the first derivatives of $\mathbf{R}$ with respect to $\mathbf{F}$,

$$
\frac{d \operatorname{vec} \mathbf{R}}{d \operatorname{vec}^{\top} \mathbf{F}}=\left(\mathbf{N}^{\top} \otimes \mathbf{I}_{n}\right)
$$

The second derivatives of $\mathbf{R}$ are given by the Hessian matrix

$$
H[\operatorname{vec} \mathbf{R} ; \operatorname{vec} \mathbf{F}]=\frac{d}{d \operatorname{vec}^{\top} \mathbf{F}} \operatorname{vec}\left[\mathbf{N} \otimes \mathbf{I}_{n}\right] .
$$

However, because $\mathbf{N}$ depends only on $\mathbf{U}$, and not on $\mathbf{F}$, this is a zero matrix.

Substituting these results into (4.48) gives

$$
H\left[R_{0} ; \operatorname{vec} \mathbf{F}\right]=\frac{1}{2}\left(\mathbf{B}+\mathbf{B}^{\mathbf{\top}}\right)
$$

where

$$
\mathbf{B}=\left(\mathbf{N} \otimes \mathbf{I}_{n}\right) H\left[R_{0} ; \operatorname{vec} \mathbf{R}\right]\left(\mathbf{N}^{\top} \otimes \mathbf{I}_{n}\right)
$$

\subsubsection{Single type of offspring}

In the common case where there is only one type of offspring (Appendix 4.C.1), $H\left[R_{0} ; \boldsymbol{\theta}\right]$ simplifies to

$$
H\left[R_{0} ; \boldsymbol{\theta}\right]=\left(\mathbf{e}_{1}^{\top} \otimes \mathbf{e}_{1}^{\top} \otimes \mathbf{I}_{s}\right) H[\operatorname{vec} \mathbf{R} ; \boldsymbol{\theta}]
$$

where $\mathbf{e}_{1}$ is the $n \times 1$ vector with 1 as its first entry and zeros elsewhere.

\subsection{Case study: Calathea ovandensis}

\subsubsection{Study species}

Calathea ovandensis is a neotropical perennial herb that inhabits forest understories. Horvitz and Schemske (1995) developed a stage-structured matrix model for C. ovandensis that contains eight stages distinguished by size and reproductive ability: seeds, nonreproductive stages (seedlings, juveniles, pre-reproductive), and reproductive stages (small, medium, large, and extra large). Plants may grow larger, remain in the same size class, or shrink at each time step; larger adults are typically more fecund.

Horvitz and Schemske summarized four years of population dynamics from four plots of $C$. ovandensis with an $8 \times 8$ summary projection matrix, the average plot-year matrix weighted by the observed stage abundances and transition frequencies in different years and plots (Horvitz and 
a)

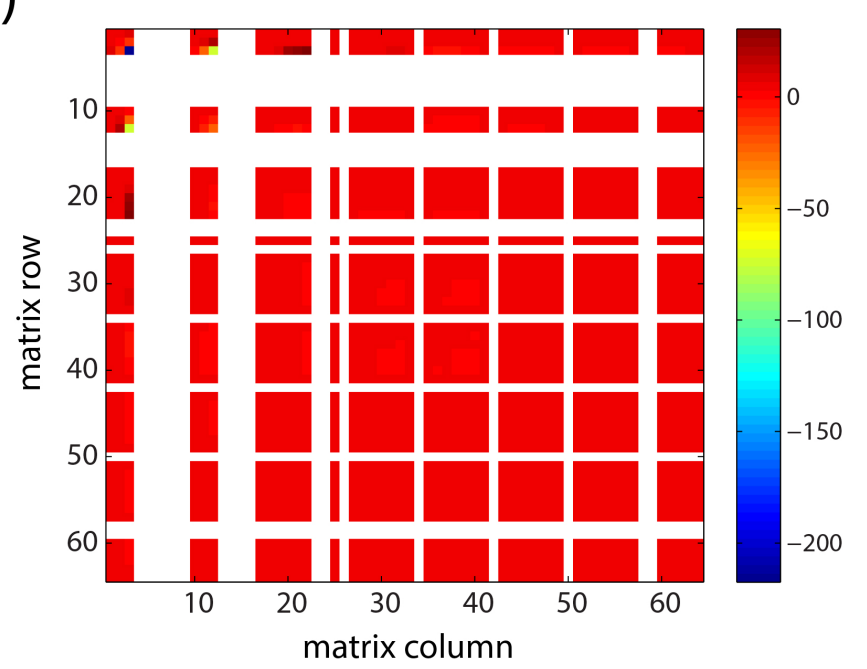

b)

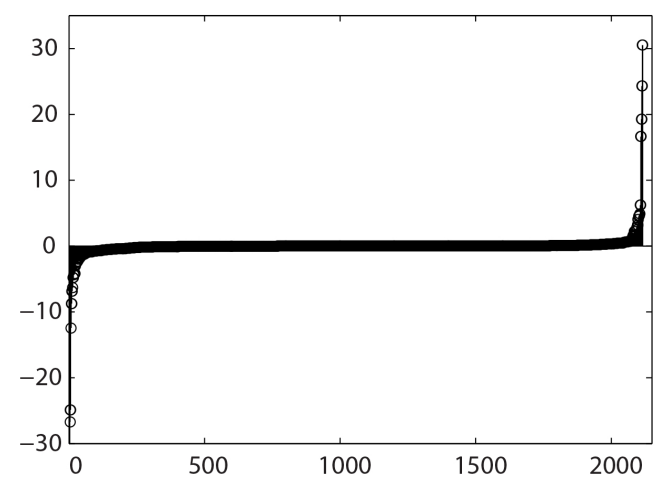

Figure 4-1: . (a) The Hessian matrix $H[\lambda ; \operatorname{vec} \mathbf{A}]$ for $C$. ovandensis. Entries corresponding to fixed zeros (unobserved transitions) in the matrix (4.59) are omitted. (b) The entries of the Hessian matrix in 4-1a, sorted in ascending order. The derivatives $\partial^{2} \lambda / \partial a_{3,1}^{2}=-217.53$ and $\partial^{2} \lambda / \partial a_{3,1} \partial a_{4,2}=$ -75.64 are omitted due to their magnitude.

Schemske 1995, Table 8):

$$
\mathbf{A}=\left(\begin{array}{cccccccc}
0.4983 & 0 & 0.5935 & 7.139 & 14.2715 & 24.6953 & 34.9027 & 40.5437 \\
0.0973 & 0.0110 & 0.0191 & 0 & 0 & 0 & 0 & 0 \\
0.0041 & 0.0442 & 0.3378 & 0.0698 & 0.0251 & 0.0065 & 0.0085 & 0 \\
0 & 0.0014 & 0.1355 & 0.4286 & 0.1736 & 0.0968 & 0.0427 & 0.0435 \\
0 & 0 & 0.0363 & 0.3841 & 0.6025 & 0.4258 & 0.2991 & 0.2174 \\
0 & 0 & 0.0019 & 0.0254 & 0.113 & 0.2387 & 0.1709 & 0.2826 \\
0 & 0 & 0 & 0.0095 & 0.0272 & 0.1548 & 0.3248 & 0.1957 \\
0 & 0 & 0 & 0.0032 & 0.0063 & 0.0452 & 0.1282 & 0.2391
\end{array}\right)
$$

The dominant eigenvalue of this matrix is 0.9923 , indicating a near-steady state population.

\subsubsection{Second derivatives of $\lambda$}

The Hessian $H[\lambda ; \operatorname{vec} \mathbf{A}]$, which is calculated using (4.29), gives the second derivatives of $\lambda$ to the entries of $\mathbf{A}$. It is a symmetric $64 \times 64$ matrix (Figure $4-1$ ). In this example, and in others with large projection matrices, $H[\lambda$; vec $\mathbf{A}]$ contains many entries and may be difficult to interpret, even when entries that are fixed at 0 are omitted. Most of the second derivatives here are small in magnitude (Figure 4-1b) with the exception of a few entries, including the highly-negative $\partial^{2} \lambda / \partial a_{3,1}^{2}=-217.53$ and $\partial^{2} \lambda / \partial a_{3,1} \partial a_{4,2}=-75.64$, where $a_{3,1}$ is the transition probability from seed to juvenile, whereas $a_{4,2}$ is the transition probability from seedling to pre-reproductives. 
Using (4.40), one can also calculate the Hessian $H[\lambda ; \boldsymbol{\theta}]$ for a set of lower-level parameters $\boldsymbol{\theta}$. For example, the stage-specific survival probabilities are lower-level parameters that affect multiple matrix entries. To analyze these using (4.40), write the survival probabilities in a vector $\boldsymbol{\sigma}$, which is given by the column sums of $\mathbf{U}$, so that

$$
\mathbf{U}=\mathbf{G} \operatorname{diag}(\boldsymbol{\sigma})
$$

where $\mathbf{G}$ describes stage transitions conditional on survival (Caswell 2011).

The Hessian of $\lambda$ with respect to $\boldsymbol{\sigma}$ is given by (4.40), with the parameter vector $\boldsymbol{\theta}$ replaced by $\boldsymbol{\sigma}$. Calculating this Hessian requires the first and second derivatives of $\mathbf{A}$ with respect to $\boldsymbol{\sigma}$. These are obtained as follows. Assuming that $\mathbf{F}$ does not depend on $\boldsymbol{\sigma}$ (i.e., prebreeding census), the first differential of $\mathbf{A}$ is

$$
d \mathbf{A}=d \mathbf{U}=\mathbf{G} \operatorname{diag}(d \boldsymbol{\sigma})
$$

Applying the vec operator to both sides gives

$$
\frac{d \operatorname{vec} \mathbf{A}}{d \boldsymbol{\sigma}^{\top}}=\left(\mathbf{I}_{n} \otimes \mathbf{G}\right) \operatorname{diag}\left(\operatorname{vec} \mathbf{I}_{n}\right)\left(\mathbf{e} \otimes \mathbf{I}_{n}\right)
$$

(see Caswell and Salguero-Gómez 2013, Appendix A).

The Hessian $H[\operatorname{vec} \mathbf{A} ; \boldsymbol{\sigma}]$ is the derivative of (4.62) with respect to $\boldsymbol{\theta}$. However, none of the terms in (4.62) depend on $\boldsymbol{\sigma}$, so the second derivatives of $\mathbf{A}$ with respect to $\boldsymbol{\sigma}$ are all zero, and $H[\operatorname{vec} \mathbf{A} ; \boldsymbol{\sigma}]$ is a zero matrix. Thus, the matrix $\mathbf{B}$ in (4.40) reduces to

$$
\mathbf{B}=\left(\frac{d \operatorname{vec} \mathbf{A}}{d \boldsymbol{\sigma}^{\top}}\right)^{\top} H[\lambda ; \operatorname{vec} \mathbf{A}] \frac{d \operatorname{vec} \mathbf{A}}{d \boldsymbol{\sigma}^{\top}}
$$

where $\frac{d \mathrm{vec} \mathbf{A}}{d \boldsymbol{\sigma} \boldsymbol{T}}$ is given by (4.62) and $H[\lambda ; \operatorname{vec} \mathbf{A}]$ is given by (4.29).

The resulting Hessian matrix $H[\lambda ; \boldsymbol{\sigma}]$ is shown in Figure 4-2. These second derivatives are generally of smaller magnitude than those of $H[\lambda$; vec $\mathbf{A}]$ (Figure 4-1). The largest second derivatives in $H[\lambda ; \boldsymbol{\sigma}]$ appear in rows 1 and 2 (equivalently, columns 1 and 2). Figure 4-3 highlights the mixed second derivatives $\partial^{2} \lambda / \partial \sigma_{1} \partial \sigma_{i}$ and $\partial^{2} \lambda / \partial \sigma_{2} \partial \sigma_{i}$, along with the pure second derivatives $\partial^{2} \lambda / \partial \sigma_{i}^{2}$.

\subsubsection{Applications of the second derivatives}

C. ovandensis has several large second derivatives of the form $\partial^{2} \lambda / \partial \sigma_{1} \partial \sigma_{i}$ and $\partial^{2} \lambda / \partial \sigma_{2} \partial \sigma_{i}$ (the first two rows or columns of Figure 4-2, which are shown separately in Figures 4-3b and 4-3c). As discussed in Section 4.2.1.1, this indicates that the sensitivity of $\lambda$ to stage 1 (seed) and stage 2 (seedling) survival will be especially responsive to changes in later survival. Similarly, the sensitivity of $\lambda$ to later survival is especially responsive to changes in seed and seedling survival.

Recall from Section 4.2.1.2 that if $\partial^{2} \lambda / \partial \theta^{2}=0$, the corresponding selection process is linear and can only shift the mean of trait $\theta$. In $C$. ovandensis, selection on survival in stage 8 is almost linear, 
a)

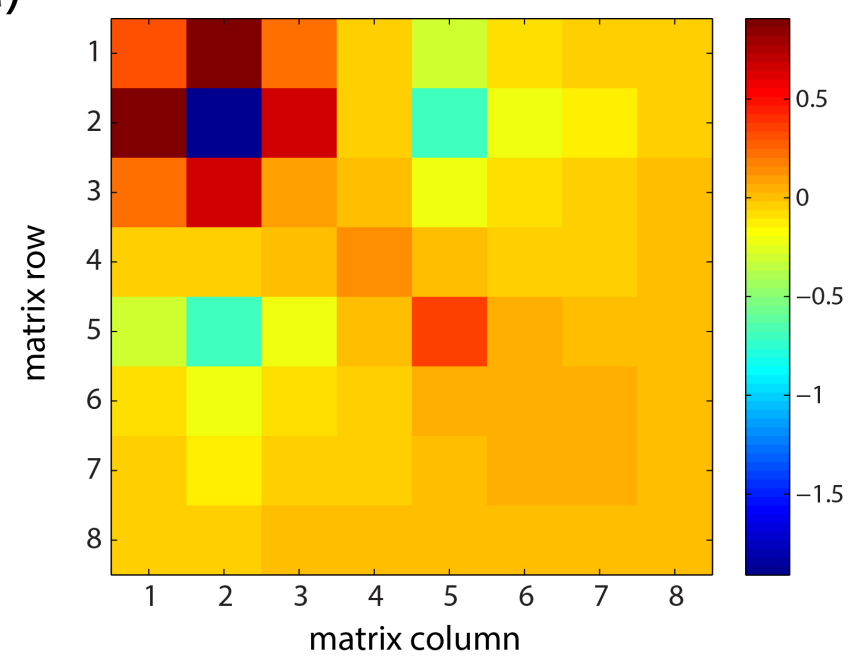

b)

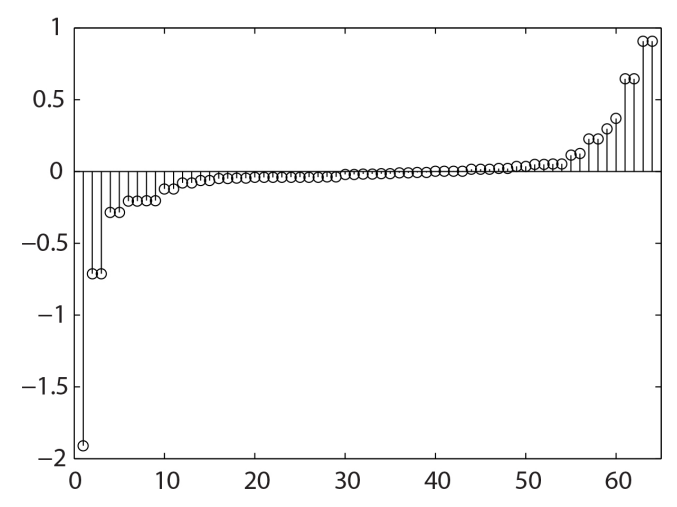

Figure 4-2: . (a) The Hessian $H[\lambda ; \boldsymbol{\sigma}]$ for C. ovandensis. (b) The Hessian entries in 4-2a, sorted in ascending order.
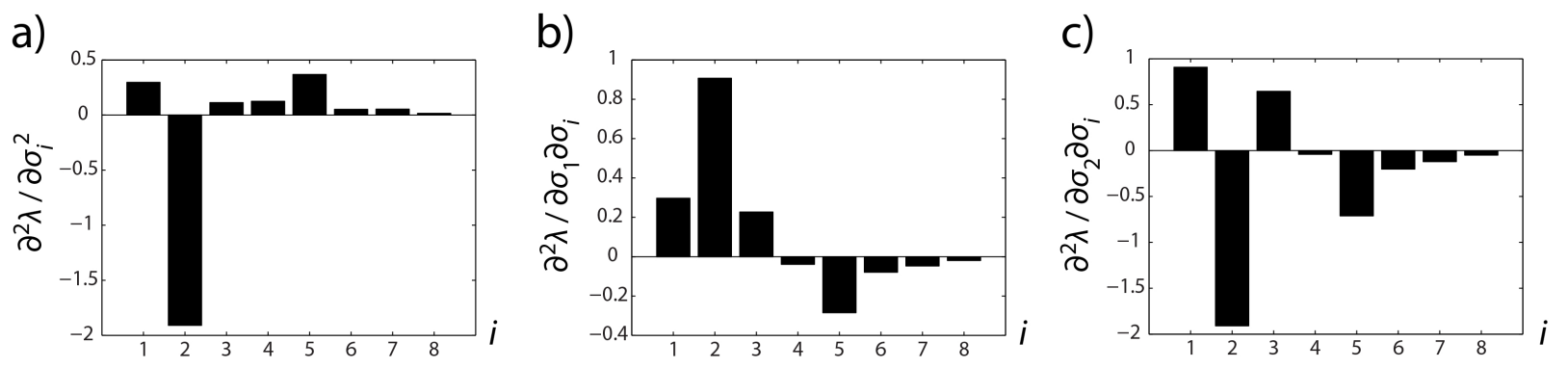

Figure 4-3: . Three sets of second derivatives from $H[\lambda ; \boldsymbol{\sigma}]$ (Figure 4-2). (a) The pure second derivatives $\partial^{2} \lambda / \partial \sigma_{i}^{2}$. (b) The mixed second derivatives $\partial^{2} \lambda / \partial \sigma_{1} \partial \sigma_{i}$. (c) The mixed second derivatives $\partial^{2} \lambda / \partial \sigma_{2} \partial \sigma_{i}$. 
since $\partial^{2} \lambda / \partial \sigma_{8}^{2}$ is near zero. If $\partial^{2} \lambda / \partial \theta^{2}>0$, selection is convex and can increase trait variance (e.g., survival in stages $1,3,4,5)$. If $\partial^{2} \lambda / \partial \theta^{2}<0$, selection is concave and can reduce trait variance (e.g., survival in stage 2). Survival in stage 1 (seeds) or 2 (seedlings) has negative mixed derivatives with survival in stages 4-8 (adults), but largely positive mixed derivatives with survival in stages 1-3 (pre-adults). This indicates that seed and seedling survival are being selected to decrease their correlation with adult survival, but to increase their correlation with pre-adult survival.

\subsection{Discussion}

Although the first derivatives of population growth rates are commonly used in ecology and demography, tools for calculating the second derivatives are not nearly as well-established, even though second derivatives also have a variety of potential applications. To this end, we have derived new, more easily computable formulae for the second derivatives of three population growth rate measures - the discrete-time growth rate $\lambda$, the continuous-time growth rate $r$, and the per-generation growth rate $R_{0}$ - both with respect to projection matrix entries and to lower-level parameters. Table 4.1 provides an overview of the results, with directions to the equations defining the Hessian matrix, containing all second-order partial derivatives, for each type of growth rate and each type of independent variable.

The matrix calculus approach is comprehensive, and even though the formulae may appear complicated, they are easy to apply with any matrix-oriented software. Other methods for finding second derivatives are either more limited or require more difficult and error-prone calculations. Cohen (1978), for instance, derives the second pure derivatives of $\lambda$ with respect to the diagonal elements of the projection matrix $\left(\partial^{2} \lambda / \partial a_{i i}^{2}\right)$ only. The approaches of Deutsch and Neumann (1984) and Kirkland and Neumann (1994) rely on the calculation of group inverses, while those of Caswell (1996) require all the eigenvalues and eigenvectors of the projection matrix. McCarthy et al.'s method (2008) uses transfer functions rather than eigenvectors, and is more complicated when handling lower-level parameters.

The methods described in this paper focus on calculating partial second derivatives. The total (integrated) second derivative depends on both pure and mixed second partials, as well as the derivatives of the other parameters to the parameter of interest:

$$
\frac{d^{2} \lambda}{d \theta_{i}^{2}}=\sum_{j} \frac{\partial^{2} \lambda}{\partial \theta_{j}^{2}}\left(\frac{d \theta_{j}^{2}}{d \theta_{i}}\right)^{2}+\sum_{j} \frac{\partial \lambda}{\partial \theta_{j}}\left(\frac{d^{2} \theta_{j}}{d \theta_{i}^{2}}\right)+2 \sum_{j} \frac{\partial^{2} \lambda}{\partial \theta_{i} \partial \theta_{j}} \frac{d \theta_{j}}{d \theta_{i}}
$$

This total derivative may be of particular interest when there are significant covariances between parameters, as in the case of strong life cycle trade-offs (e.g., van Tienderen 1995). 


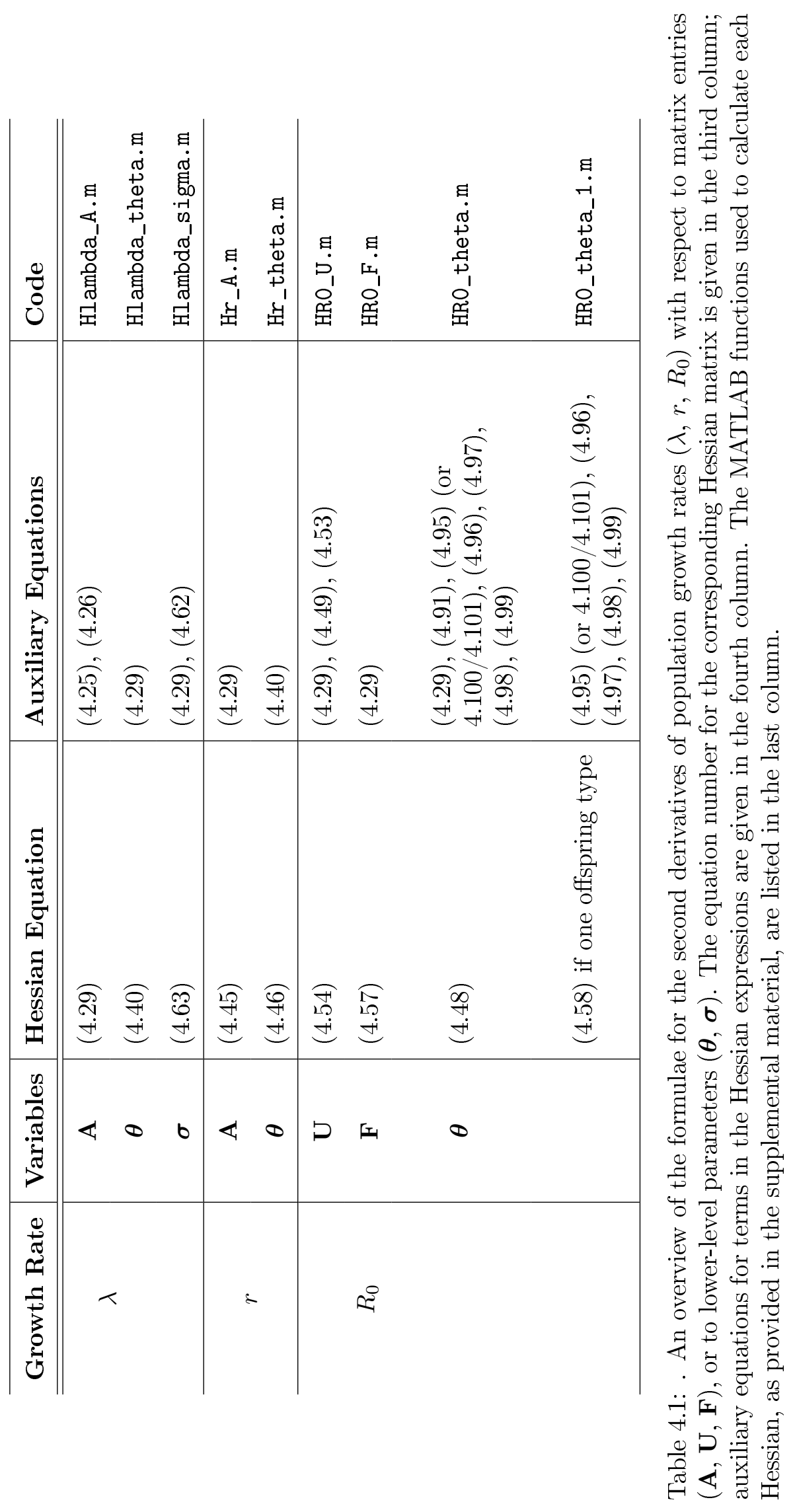




\subsection{Acknowledgments}

This work was supported by a National Science Foundation Graduate Research Fellowship under Grant 1122374, by NSF Grant DEB-1145017, and by Advanced Grant 322989 from the European Research Council.

\section{Bibliography}

[1] Brodie ED, Moore AJ, Janzen FJ. 1995. Visualizing and quantifying natural selection. Trends in Ecology and Evolution. 10: 313-318.

[2] Brommer JE. 2000. The evolution of fitness in life-history theory. Biological Reviews. 75: 377-404.

[3] Carslake D, Townley S, Hodgson DJ. 2008. Nonlinearity in eigenvalue-perturbation curves of simulated population projection matrices. Theoretical Population Biology. 73: 498-505.

[4] Caswell H. 1996. Second derivatives of population growth rate: calculation and applications. Ecology. 77: 870-879.

[5] Caswell H. 2001. Matrix population models. Sunderland, MA: Sinauer Associates, Inc.

[6] Caswell H. 2007. Sensitivity analysis of transient population dynamics. Ecology Letters. 10: $1-15$.

[7] Caswell H. 2008. Perturbation analysis of nonlinear matrix population models. Demographic Research. 18: 59-116.

[8] Caswell H. 2009. Stage, age and individual stochasticity in demography. Oikos. 118: 1763-1782.

[9] Caswell H. 2011. Matrix models and sensitivity analysis of populations classified by age and stage: a vec-permutation matrix approach. Theoretical Ecology. 5: 403-417.

[10] Caswell H, Salgueró-Gomez R. 2013. Age, stage and senescence in plants. Journal of Ecology. 101: 585-595.

[11] Cohen JE. 1978. Derivatives of the spectral radius as a function of non-negative matrix elements. Mathematical Proceedings of the Cambridge Philosophical Society. 83: 183-190.

[12] Cushing JM, Zhou Y. 1994. The net reproductive value and stability in matrix population models. Natural Resources Modeling. 8: 297-333.

[13] Deutsch E, Neumann M. 1984. Derivatives of the Perron root of an essentially nonnegative matrix and the group inverse of an M-matrix. Journal of Mathematical Analysis and Applications. 102: 1-29.

[14] Diekmann O. 2004. A beginner's guide to adaptive dynamics. Banach Center Publications. 63: $47-86$.

[15] Doebeli M. 2011. Adaptive diversification (MPB-48). Monographs in Population Biology. Princeton: Princeton University Press. 
[16] Geritz SAH, Kidsi É, Meszéna G, Metz JAJ. 1998. Evolutionarily singular strategies and the adaptive growth and branching of the evolutionary tree. Evolutionary Ecology. 12: 35-57.

[17] Henderson HV, Searle SR. 1981. The vec-permutation, the vec operator and Kronecker products: a review. Linear Multilinear Algebra. 9: 271-288.

[18] Horvitz CC, Schemske DW. 1995. Spatiotemporal variation in demographic transitions of a tropical understory herb: projection matrix analysis. Ecological Monographs. 65: 155-192.

[19] Kirkland SJ, Neumann M. 1994. Convexity and concavity of the Perron root and vector of Leslie matrices with applications to a population model. SIAM Journal of Matrix Analysis and Applications. 15: 1092-1107.

[20] Lande R, Arnold SJ. 1983. The measurement of selection on correlated characters. Evolution. 37: $1210-1226$.

[21] Magnus JR. 2007. Matrix calculus and econometrics using differentials. In D. Belsley and E. Kontoghiorghes (Eds.), Handbook on Computational Econometrics. New York: Marcel Dekker.

[22] Magnus JR, Neudecker H. 1979. The commutation matrix: some properties and applications. Annals of Statistics. 7: 381-394.

[23] Magnus JR, Neudecker, H. 1985. Matrix differential calculus with applications to simple, Hadamard, and Kronecker products. Journal of Mathematical Psychology. 29: 474-492.

[24] Magnus JR, Neudecker H. 1999. Matrix differential calculus with applications in statistics and econometrics. John Wiley and Sons Ltd.

[25] Magnus JR. 2010. On the concept of matrix derivative. Journal of Multivariate Analysis. 101: 2200-2206.

[26] McCarthy D, Townley S, Hodgson D. 2008. On second order sensitivity for stage-based population projection matrix models. Theoretical Population Biology. 74: 68-73.

[27] Pásztor L, Meszéna G, Kidsi É. 1996. $R_{0}$ or $r$ : a matter of taste? Journal of Evolutionary Biology. 9: 511-518.

[28] Phillips PC, Arnold SJ. 1989. Visualizing multivariate selection. Evolution. 43: 1209-1222.

[29] Roth WE. 1934. On direct product matrices. Bulletin of the American Mathematical Society. 40: 461-468.

[30] Stearns SC. 1992. The evolution of life histories. Oxford, England: Oxford University Press.

[31] Tuljapurkar SD. 1982. Population dynamics in variable environments. III. Evolutionary dynamics of $r$-selection. Theoretical Population Biology. 21: 141-165.

[32] van Tienderen PH. 1995. Life cycle trade-offs in matrix population models. Ecology. 76: $2482-2489$.

[33] Waxman D, Gavrilets S. 2005. 20 questions on adaptive dynamics. Journal of Evolutionary Biology. 18: 1139-1154. 


\section{Chapter 4 Appendix}

In these appendices, we provide derivations of the sensitivity results. For clarity, we provide some derivations that were originally obtained in other publications.

\section{A First differentials of $\lambda, w$, and $v$}

Here, we present derivations for the first differentials of the dominant eigenvalue $\lambda$ and its right and left eigenvectors $\mathbf{w}$ and $\mathbf{v}$. Eigenvectors are written as $n \times 1$ column vectors and scaled so that $\mathbf{v}^{\top} \mathbf{w}=1$ and $\mathbf{e}^{\top} \mathbf{w}=1$, where $\mathbf{e}$ is a $n \times 1$ vector of ones.

\section{A.1 First differential of the dominant eigenvalue: $d \lambda$}

Caswell (2010) derived the matrix calculus version of the sensitivity of $\lambda$ with respect to the entries of $\mathbf{A}$. For a matrix $\mathbf{A}$ with eigenvalue $\lambda$ and corresponding right eigenvector $\mathbf{w}$ :

$$
\mathbf{A w}=\lambda \mathbf{w}
$$

Differentiate once, then multiply each term by the transpose of the left eigenvector $\mathbf{v}^{\top}$ :

$$
\mathbf{v}^{\top}(d \mathbf{A}) \mathbf{w}+\mathbf{v}^{\top} \mathbf{A}(d \mathbf{w})=(d \lambda) \mathbf{v}^{\top} \mathbf{w}+\lambda \mathbf{v}^{\top}(d \mathbf{w})
$$

By the definition of the left eigenvector, $\mathbf{v}^{\top} \mathbf{A}=\lambda \mathbf{v}^{\top}$. After canceling terms and rearranging the resulting expression, we find:

$$
d \lambda=\frac{\mathbf{v}^{\top}(d \mathbf{A}) \mathbf{w}}{\mathbf{v}^{\top} \mathbf{w}} .
$$

Scaling $\mathbf{v}^{\top} \mathbf{w}=1$ and applying Roth's theorem (4.6):

$$
\begin{aligned}
d \lambda & =\mathbf{v}^{\top}(d \mathbf{A}) \mathbf{w} \\
& =\left(\mathbf{w}^{\boldsymbol{\top}} \otimes \mathbf{v}^{\boldsymbol{\top}}\right) d \operatorname{vec} \mathbf{A} .
\end{aligned}
$$




\section{A.2 First differential of the right eigenvector: $d w$}

As shown in Caswell (2008), when the right eigenvector is scaled so that $\mathbf{e}^{\top} \mathbf{w}=1, \mathbf{w}$ can be written as:

$$
\mathbf{w}=\frac{\mathbf{A w}}{\mathbf{e}^{\top} \mathbf{A w}}
$$

Differentiate using the quotient rule,

$$
\mathbf{w}=\frac{1}{\left(\mathbf{e}^{\top} \mathbf{A} \mathbf{w}\right)^{2}}\left[\mathbf{e}^{\top} \mathbf{A} \mathbf{w} d(\mathbf{A} \mathbf{w})-\mathbf{A} \mathbf{w} d\left(\mathbf{e}^{\top} \mathbf{A} \mathbf{w}\right)\right]
$$

then substitute $\mathbf{A} \mathbf{w}=\lambda \mathbf{w}$ and $\mathbf{e}^{\top} \mathbf{A} \mathbf{w}=\lambda$ to obtain

$$
\begin{aligned}
\lambda d \mathbf{w} & =\left(\mathbf{I}_{n}-\mathbf{w} \mathbf{e}^{\boldsymbol{\top}}\right) d(\mathbf{A} \mathbf{w}) \\
& =\left(\mathbf{I}_{n}-\mathbf{w} \mathbf{e}^{\boldsymbol{\top}}\right)(d \mathbf{A}) \mathbf{w}+\left(\mathbf{A}-\mathbf{w} \mathbf{e}^{\boldsymbol{\top}} \mathbf{A}\right) d \mathbf{w}
\end{aligned}
$$

Apply the vec operator and rearrange terms,

$$
\left(\lambda \mathbf{I}_{n}-\mathbf{A}+\mathbf{w e}^{\top} \mathbf{A}\right) d \mathbf{w}=\left[\mathbf{w}^{\boldsymbol{\top}} \otimes\left(\mathbf{I}_{n}-\mathbf{w e}^{\top}\right)\right] d \operatorname{vec} \mathbf{A}
$$

then solve for $d \mathbf{w}$ to obtain

$$
d \mathbf{w}=\left(\lambda \mathbf{I}_{n}-\mathbf{A}+\mathbf{w e}^{\top} \mathbf{A}\right)^{-1}\left[\mathbf{w}^{\top} \otimes\left(\mathbf{I}_{n}-\mathbf{w e}^{\top}\right)\right] d \operatorname{vec} \mathbf{A}
$$

\section{A.3 First differential of the left eigenvector: $d v$}

By scaling $\mathbf{w}^{\top} \mathbf{v}=\mathbf{v}^{\top} \mathbf{w}=1$, the left eigenvector $\mathbf{v}$ can be written as:

$$
\mathbf{v}^{\top}=\frac{\mathbf{v}^{\top} \mathbf{A}}{\mathbf{v}^{\top} \mathbf{A} \mathbf{w}}
$$

(Caswell and Vindenes, in prep.). Differentiate using the quotient rule and note that $\mathbf{A w}=\lambda \mathbf{w}$ and $v^{\top} \mathbf{A}=\lambda \mathbf{v}^{\top}$ :

$$
\begin{aligned}
d \mathbf{v}^{\top} & =\frac{\left(\mathbf{v}^{\top} \mathbf{A} \mathbf{w}\right) d\left(\mathbf{v}^{\top} \mathbf{A}\right)-d\left(\mathbf{v}^{\top} \mathbf{A} \mathbf{w}\right)\left(\mathbf{v}^{\top} \mathbf{A}\right)}{\left(\mathbf{v}^{\top} \mathbf{A} \mathbf{w}\right)^{2}} \\
& =\frac{1}{\left(\lambda \mathbf{v}^{\top} \mathbf{w}\right)^{2}}\left[\lambda \mathbf{v}^{\top} \mathbf{w}\left[\left(d \mathbf{v}^{\top}\right) \mathbf{A}+\mathbf{v}^{\top}(d \mathbf{A})\right]-\left[\left(d \mathbf{v}^{\top}\right) \lambda \mathbf{w}+\mathbf{v}^{\top}(d \mathbf{A}) \mathbf{w}+\lambda \mathbf{v}^{\top}(d \mathbf{w})\right]\left(\lambda \mathbf{v}^{\top}\right)\right]
\end{aligned}
$$

Multiplying both sides by $\lambda$ and setting $\mathbf{v}^{\top} \mathbf{w}=1$ :

$$
\lambda d \mathbf{v}^{\top}=\left(d \mathbf{v}^{\top}\right) \mathbf{A}+\mathbf{v}^{\top}(d \mathbf{A})-\lambda\left(d \mathbf{v}^{\top}\right) \mathbf{w} \mathbf{v}^{\top}-\mathbf{v}^{\top}(d \mathbf{A}) \mathbf{w} \mathbf{v}^{\top}-\lambda \mathbf{v}^{\top}(d \mathbf{w}) \mathbf{v}^{\top}
$$


Take the vec of both sides and apply Roth's theorem (4.6),

$$
\begin{aligned}
\lambda d \mathbf{v} & =\left(\mathbf{A}^{\boldsymbol{\top}} \otimes \mathbf{I}_{1}\right) d \mathbf{v}+\left(\mathbf{I}_{n} \otimes \mathbf{v}^{\boldsymbol{\top}}\right) d \operatorname{vec} \mathbf{A}-\lambda\left(\mathbf{v} \mathbf{w}^{\top} \otimes \mathbf{I}_{1}\right) d \mathbf{v}-\left(\mathbf{v} \mathbf{w}^{\top} \otimes \mathbf{v}^{\top}\right) d \operatorname{vec} \mathbf{A}-\lambda\left(\mathbf{v} \otimes \mathbf{v}^{\top}\right) d \mathbf{w} \\
& =\mathbf{A}^{\top} d \mathbf{v}+\left(\mathbf{I}_{n} \otimes \mathbf{v}^{\boldsymbol{\top}}\right) d \operatorname{vec} \mathbf{A}-\lambda \mathbf{v} \mathbf{w}^{\boldsymbol{\top}} d \mathbf{v}-\left(\mathbf{v} \mathbf{w}^{\top} \otimes \mathbf{v}^{\boldsymbol{\top}}\right) d \operatorname{vec} \mathbf{A}-\lambda\left(\mathbf{v} \otimes \mathbf{v}^{\boldsymbol{\top}}\right) d \mathbf{w}
\end{aligned}
$$

then solve for $d \mathbf{v}$ to obtain

$$
d \mathbf{v}=\left(\lambda \mathbf{I}_{n}-\mathbf{A}^{\top}+\lambda \mathbf{v} \mathbf{w}^{\top}\right)^{-1}\left[\left(\left[\mathbf{I}_{n}-\mathbf{v} \mathbf{w}^{\top}\right] \otimes \mathbf{v}^{\top}\right) d \operatorname{vec} \mathbf{A}-\lambda\left(\mathbf{v} \otimes \mathbf{v}^{\top}\right) d \mathbf{w}\right]
$$

\section{B First derivatives of $\mathbf{S}=\frac{d \mathbf{v e c} \mathbf{A}}{d \boldsymbol{\theta}^{\top}}$ and $\mathbf{T}=\frac{d \lambda}{d \mathbf{v e c}^{\top} \mathbf{A}}$}

In (4.34), we defined

$$
\mathbf{S}=\frac{d \operatorname{vec} \mathbf{A}}{d \boldsymbol{\theta}^{\top}} \quad \text { and } \quad \mathbf{T}=\frac{d \lambda}{d \operatorname{vec}^{\top} \mathbf{A}}=\mathbf{w}^{\top} \otimes \mathbf{v}^{\top}
$$

To evaluate (4.36), we require the first derivatives of $\mathbf{S}$ and $\mathbf{T}$. For the first derivative of $\mathbf{S}$ :

$$
\frac{d \mathrm{vec} \mathbf{S}}{d \boldsymbol{\theta}^{\top}}=\frac{d}{d \boldsymbol{\theta}^{\top}} \operatorname{vec}\left[\frac{d \mathrm{vec} \mathbf{A}}{d \boldsymbol{\theta}^{\top}}\right]
$$

Apply the commutation matrix (vec-permutation matrix) $\mathbf{K}_{m, n}$, a constant matrix of 0's and 1's that can be calculated using the MATLAB function provided in Appendix 4.E. For any $m \times n$ matrix $\mathbf{A}$, there is a unique $m n \times m n$ matrix $\mathbf{K}_{m, n}$ such that:

$$
\mathbf{K}_{m, n} \operatorname{vec} \mathbf{A}=\operatorname{vec}\left(\mathbf{A}^{\top}\right)
$$

Using both the commutation matrix and the definition of the Hessian matrix in (4.13):

$$
\begin{aligned}
\frac{d \operatorname{vec} \mathbf{S}}{d \boldsymbol{\theta}^{\top}} & =\frac{d}{d \boldsymbol{\theta}^{\top}} \operatorname{vec}\left[\frac{d \operatorname{vec} \mathbf{A}}{d \boldsymbol{\theta}^{\top}}\right] \\
& =\frac{d}{d \boldsymbol{\theta}^{\top}} \mathbf{K}_{s, n^{2}} \operatorname{vec}\left[\left(\frac{d \operatorname{vec} \mathbf{A}}{d \boldsymbol{\theta}^{\top}}\right)^{\top}\right] \\
& =\mathbf{K}_{s, n^{2}} H[\operatorname{vec} \mathbf{A} ; \boldsymbol{\theta}] .
\end{aligned}
$$

Note that for a $m \times n$ matrix $\mathbf{X}$ and a $p \times q$ matrix $\mathbf{Y}$,

$$
\mathbf{K}_{p, m}(\mathbf{X} \otimes \mathbf{Y})=(\mathbf{Y} \otimes \mathbf{X}) \mathbf{K}_{q, n}
$$

and

$$
\mathbf{K}_{n, 1}=\mathbf{K}_{1, n}=\mathbf{I}_{n}
$$


so (4.37) becomes

$$
\begin{aligned}
\left(\mathbf{I}_{s} \otimes \mathbf{T}\right) \frac{d \operatorname{vec} \mathbf{S}}{d \boldsymbol{\theta}^{\top}} & =\left(\mathbf{I}_{s} \otimes \mathbf{T}\right) \mathbf{K}_{s, n^{2}} H[\operatorname{vec} \mathbf{A} ; \boldsymbol{\theta}] \\
& =\left(\mathbf{T} \otimes \mathbf{I}_{s}\right) H[\operatorname{vec} \mathbf{A} ; \boldsymbol{\theta}] .
\end{aligned}
$$

To find (4.38), differentiate the expression for $\mathbf{T}$

$$
\frac{d \operatorname{vec} \mathbf{T}}{d \boldsymbol{\theta}^{\top}}=\frac{d}{d \boldsymbol{\theta}^{\top}} \operatorname{vec}\left[\frac{d \lambda}{d \operatorname{vec} \mathbf{A}^{\top}}\right]
$$

and use the chain rule to rewrite it in terms of the derivative with respect to $\mathbf{A}$

$$
\frac{d \operatorname{vec} \mathbf{T}}{d \boldsymbol{\theta}^{\top}}=\frac{d}{d \operatorname{vec} \mathbf{A}^{\top}} \operatorname{vec}\left[\frac{d \lambda}{d \operatorname{vec} \mathbf{A}^{\top}}\right] \frac{d \operatorname{vec} \mathbf{A}}{d \boldsymbol{\theta}^{\top}} .
$$

The first part of (4.88) is equivalent to the Hessian expression (4.13), so:

$$
\frac{d \operatorname{vec} \mathbf{T}}{d \boldsymbol{\theta}^{\top}}=H[\lambda ; \operatorname{vec} \mathbf{A}] \mathbf{S}
$$

\section{C Second derivatives of $R_{0}$ to lower-level parameters: $H\left[R_{0} ; \boldsymbol{\theta}\right]$}

In Section 4.4.3, we calculated the second derivatives of the net reproductive rate $R_{0}$ with respect to $\mathbf{U}$ and $\mathbf{F}$. Here, we present the fully general second derivatives of $R_{0}$ with respect to a parameter vector $\boldsymbol{\theta}$.

As written in (4.48), the general expression for the Hessian of $R_{0}$ with respect to $\boldsymbol{\theta}$ is

$$
H\left[R_{0} ; \boldsymbol{\theta}\right]=\frac{1}{2}\left(\mathbf{B}+\mathbf{B}^{\top}\right)
$$

where

$$
\mathbf{B}=\left(\mathbf{w}_{R}^{\top} \otimes \mathbf{v}_{R}^{\top} \otimes \mathbf{I}_{s}\right) H[\operatorname{vec} \mathbf{R} ; \boldsymbol{\theta}]+\left(\frac{d \mathrm{vec} \mathbf{R}}{d \boldsymbol{\theta}^{\top}}\right)^{\top} H\left[R_{0} ; \operatorname{vec} \mathbf{R}\right] \frac{d \operatorname{vec} \mathbf{R}}{d \boldsymbol{\theta}^{\top}}
$$

and $\mathbf{w}_{R}$ and $\mathbf{v}_{R}$ are the right and left eigenvectors of $\mathbf{R}$. As noted in the main text, evaluating $\mathbf{B}$ requires the second derivatives of $R_{0}$ with respect to $\mathbf{R}$ (the Hessian $H\left[R_{0} ; \operatorname{vec} \mathbf{R}\right]$, which is given by (4.29) using the dominant eigenvalues and eigenvectors of $\mathbf{R}$ rather than those of $\mathbf{A}$ ), and the first and second derivatives of $\mathbf{R}$ with respect to $\boldsymbol{\theta}$. We will now calculate these derivatives of $\mathbf{R}$ with respect to $\boldsymbol{\theta}$.

The first derivatives of $\mathbf{R}$ with respect to $\boldsymbol{\theta}$ are

$$
\frac{d \operatorname{vec} \mathbf{R}}{d \boldsymbol{\theta}^{\top}}=\left(\mathbf{N}^{\top} \otimes \mathbf{I}_{n}\right) \frac{d \operatorname{vec} \mathbf{F}}{d \boldsymbol{\theta}^{\top}}+\left(\mathbf{N}^{\top} \otimes \mathbf{R}\right) \frac{d \operatorname{vec} \mathbf{U}}{d \boldsymbol{\theta}^{\top}} .
$$


The second derivatives of $\mathbf{R}$ with respect to $\boldsymbol{\theta}$ are given by the definition of the Hessian matrix (4.13):

$$
\begin{aligned}
H[\operatorname{vec} \mathbf{R} ; \boldsymbol{\theta}] & =\frac{d}{d \boldsymbol{\theta}^{\top}} \operatorname{vec}\left[\left(\frac{d \operatorname{vec} \mathbf{R}}{d \boldsymbol{\theta}^{\top}}\right)^{\top}\right] \\
& =\frac{d}{d \boldsymbol{\theta}^{\top}} \operatorname{vec}\left[\left(\frac{d \operatorname{vec} \mathbf{F}}{d \boldsymbol{\theta}^{\top}}\right)^{\top}\left(\mathbf{N} \otimes \mathbf{I}_{n}\right)+\left(\frac{d \operatorname{vec} \mathbf{U}}{d \boldsymbol{\theta}^{\top}}\right)^{\top}\left(\mathbf{N} \otimes \mathbf{R}^{\top}\right)\right] .
\end{aligned}
$$

To evaluate this expression, we will use the following rule (Magnus 2010) for derivatives of matrix products: given matrices $\mathbf{Y}(n \times v)$ and $\mathbf{X}(m \times n)$, the derivative of their product with respect to a third matrix $\mathbf{Z}(p \times q)$ is

$$
\frac{d \operatorname{vec}(\mathbf{X Y})}{d(\operatorname{vec} \mathbf{Z})^{\top}}=\left(\mathbf{Y}^{\boldsymbol{\top}} \otimes \mathbf{I}_{m}\right) \frac{d \operatorname{vec} \mathbf{X}}{d(\operatorname{vec} \mathbf{Z})^{\top}}+\left(\mathbf{I}_{v} \otimes \mathbf{X}\right) \frac{d \operatorname{vec} \mathbf{Y}}{d(\operatorname{vec} \mathbf{Z})^{\top}}
$$

Applying this product rule for matrix derivatives to (4.92) gives

$$
\begin{aligned}
H[\operatorname{vec} \mathbf{R} ; \boldsymbol{\theta}]= & {\left[\left(\mathbf{N} \otimes \mathbf{I}_{n}\right)^{\boldsymbol{\top}} \otimes \mathbf{I}_{s}\right] \frac{d}{d \boldsymbol{\theta}^{\boldsymbol{\top}}} \operatorname{vec}\left[\left(\frac{d \operatorname{vec} \mathbf{F}}{d \boldsymbol{\theta}^{\boldsymbol{\top}}}\right)^{\top}\right]+\left[\mathbf{I}_{n^{2}} \otimes\left(\frac{d \operatorname{vec} \mathbf{F}}{d \boldsymbol{\theta}^{\top}}\right)^{\top}\right] \frac{d}{d \boldsymbol{\theta}^{\top}} \operatorname{vec}\left(\mathbf{N} \otimes \mathbf{I}_{n}\right) } \\
& +\left[\left(\mathbf{N} \otimes \mathbf{R}^{\boldsymbol{\top}}\right)^{\boldsymbol{\top}} \otimes \mathbf{I}_{s}\right] \frac{d}{d \boldsymbol{\theta}^{\top}} \operatorname{vec}\left[\left(\frac{d \operatorname{vec} \mathbf{U}}{d \boldsymbol{\theta}^{\top}}\right)^{\top}\right]+\left[\mathbf{I}_{n^{2}} \otimes\left(\frac{d \operatorname{vec} \mathbf{U}}{d \boldsymbol{\theta}^{\boldsymbol{\top}}}\right)^{\top}\right] \frac{d}{d \boldsymbol{\theta}^{\boldsymbol{\top}}} \operatorname{vec}\left(\mathbf{N} \otimes \mathbf{R}^{\top}\right)
\end{aligned}
$$

and recognizing Hessian matrices of the form (4.13), we obtain

$$
\begin{aligned}
H[\operatorname{vec} \mathbf{R} ; \boldsymbol{\theta}]= & \left(\mathbf{N}^{\top} \otimes \mathbf{I}_{n s}\right) H[\operatorname{vec} \mathbf{F} ; \boldsymbol{\theta}]+\left[\mathbf{I}_{n^{2}} \otimes\left(\frac{d \operatorname{vec} \mathbf{F}}{d \boldsymbol{\theta}^{\top}}\right)^{\top}\right] \frac{d}{d \boldsymbol{\theta}^{\top}} \operatorname{vec}\left(\mathbf{N} \otimes \mathbf{I}_{n}\right) \\
& +\left(\mathbf{N}^{\top} \otimes \mathbf{R} \otimes \mathbf{I}_{s}\right) H[\operatorname{vec} \mathbf{U} ; \boldsymbol{\theta}]+\left[\mathbf{I}_{n^{2}} \otimes\left(\frac{d \operatorname{vec} \mathbf{U}^{\top}}{d \boldsymbol{\theta}^{\top}}\right)^{\top}\right] \frac{d}{d \boldsymbol{\theta}^{\top}} \operatorname{vec}\left(\mathbf{N} \otimes \mathbf{R}^{\top}\right) .
\end{aligned}
$$

Applying (4.51), the derivatives of Kronecker products appearing (4.95) are

$$
\begin{aligned}
& \frac{d}{d \boldsymbol{\theta}^{\top}} \operatorname{vec}\left(\mathbf{N} \otimes \mathbf{I}_{n}\right)=\left(\mathbf{I}_{n} \otimes \mathbf{K}_{n, n} \otimes \mathbf{I}_{n}\right)\left(\mathbf{I}_{n^{2}} \otimes \operatorname{vec}\left[\mathbf{I}_{n}\right]\right) \frac{d \operatorname{vec} \mathbf{N}}{d \boldsymbol{\theta}^{\top}} \\
& \frac{d}{d \boldsymbol{\theta}^{\boldsymbol{\top}}} \operatorname{vec}\left(\mathbf{N} \otimes \mathbf{R}^{\boldsymbol{\top}}\right)=\left(\mathbf{I}_{n} \otimes \mathbf{K}_{n, n} \otimes \mathbf{I}_{n}\right)\left[\left(\mathbf{I}_{n^{2}} \otimes \operatorname{vec}\left[\mathbf{R}^{\boldsymbol{\top}}\right]\right) \frac{d \operatorname{vec} \mathbf{N}}{d \boldsymbol{\theta}^{\top}}+\left(\operatorname{vec} \mathbf{N} \otimes \mathbf{I}_{n^{2}}\right) \frac{d \operatorname{vec} \mathbf{R}^{\boldsymbol{\top}}}{d \boldsymbol{\theta}^{\boldsymbol{\top}}}\right]
\end{aligned}
$$

and note that 


$$
\begin{aligned}
\frac{d \operatorname{vec} \mathbf{N}}{d \boldsymbol{\theta}^{\top}} & =\left(\mathbf{N}^{\top} \otimes \mathbf{N}\right) \frac{d \operatorname{vec} \mathbf{U}}{d \boldsymbol{\theta}^{\top}} \\
\frac{d \operatorname{vec} \mathbf{R}^{\top}}{d \boldsymbol{\theta}^{\top}} & =\mathbf{K}_{n, n} \frac{d \operatorname{vec} \mathbf{R}}{d \boldsymbol{\theta}^{\top}}
\end{aligned}
$$

where $\frac{d \mathrm{vec} \mathbf{R}}{d \boldsymbol{\theta}^{\mathrm{T}}}$ is given by (4.91). Substitute (4.98) and (4.99) into (4.96) and (4.97), then back into (4.95) to obtain $H[\operatorname{vec} \mathbf{R} ; \boldsymbol{\theta}]$.

While the expression for $H[\operatorname{vec} \mathbf{R} ; \boldsymbol{\theta}]$ may appear complicated, note that it simplifies considerably if only one of $\mathbf{U}$ or $\mathbf{F}$ is a function of $\boldsymbol{\theta}$, so that either $\frac{d \mathrm{vec} \mathbf{F}}{d \boldsymbol{\theta}^{\mathbf{T}}}$ or $\frac{d \mathrm{vec} \mathbf{U}}{d \boldsymbol{\theta}^{\mathbf{T}}}$ is a matrix of zeros (i.e., the parameters of interest affect only fertility or transitions, but not both).

If only $\mathbf{U}$ depends on $\boldsymbol{\theta}$ :

$$
H[\operatorname{vec} \mathbf{R} ; \boldsymbol{\theta}]=\left(\mathbf{N}^{\boldsymbol{\top}} \otimes \mathbf{R} \otimes \mathbf{I}_{s}\right) H[\operatorname{vec} \mathbf{U} ; \boldsymbol{\theta}]+\left[\mathbf{I}_{n^{2}} \otimes\left(\frac{d \operatorname{vec} \mathbf{U}}{d \boldsymbol{\theta}^{\top}}\right)^{\top}\right] \frac{d}{d \boldsymbol{\theta}^{\top}} \operatorname{vec}\left(\mathbf{N} \otimes \mathbf{R}^{\top}\right)
$$

If only $\mathbf{F}$ depends on $\boldsymbol{\theta}$ :

$$
H[\operatorname{vec} \mathbf{R} ; \boldsymbol{\theta}]=\left(\mathbf{N}^{\top} \otimes \mathbf{I}_{n s}\right) H[\operatorname{vec} \mathbf{F} ; \boldsymbol{\theta}]
$$

Ultimately, substitute $H$ [vecR; $\boldsymbol{\theta}$ ] as given by (4.95) (or 4.100 and 4.101 if appropriate), and $\frac{d \mathrm{vec} \mathbf{R}}{d \boldsymbol{\theta}^{\mathrm{T}}}$ as given by (4.91), back into (4.90) to obtain $H\left[R_{0} ; \boldsymbol{\theta}\right]$.

\section{C.1 Life cycles with only one type of offspring}

In the common case where there is only one type of offspring (stage 1 ), the expression for $H\left[R_{0} ; \boldsymbol{\theta}\right]$ simplifies considerably. In this case, the fertility matrix $\mathbf{F}$ has nonzero entries only in its first row, and $\mathbf{R}=\mathbf{F N}$ is an upper triangular matrix. Then $R_{0}$, the dominant eigenvalue of $\mathbf{R}$, is the $(1,1)$ entry of $\mathbf{R}$ :

$$
R_{0}=\mathbf{e}_{1}^{\top}(\mathbf{R}) \mathbf{e}_{1}
$$

where $\mathbf{e}_{1}$ is the $n \times 1$ vector with 1 as its first entry and zeros elsewhere (Caswell 2009).

The first differential of $R_{0}$ is then

$$
\begin{aligned}
d R_{0} & =\mathbf{e}_{1}^{\top}(d \mathbf{R}) \mathbf{e}_{1} \\
& =\left(\mathbf{e}_{1}^{\top} \otimes \mathbf{e}_{1}^{\top}\right) d \operatorname{vec} \mathbf{R}
\end{aligned}
$$

By the first identification theorem (4.14),

$$
\frac{d R_{0}}{d \boldsymbol{\theta}^{\top}}=\left(\mathbf{e}_{1}^{\top} \otimes \mathbf{e}_{1}^{\top}\right) \frac{d \mathrm{vec} \mathbf{R}}{d \boldsymbol{\theta}^{\top}}
$$


and by the definition of the Hessian matrix (4.13):

$$
\begin{aligned}
H\left[R_{0} ; \boldsymbol{\theta}\right] & =\frac{d}{d \boldsymbol{\theta}^{\boldsymbol{\top}}} \operatorname{vec}\left[\left(\frac{d R_{0}}{d \boldsymbol{\theta}^{\boldsymbol{\top}}}\right)^{\top}\right] \\
& =\frac{d}{d \boldsymbol{\theta}^{\boldsymbol{\top}}} \operatorname{vec}\left[\left(\frac{d \mathrm{vec} \mathbf{R}}{d \boldsymbol{\theta}^{\top}}\right)^{\top}\left(\mathbf{e}_{1} \otimes \mathbf{e}_{1}\right)\right] .
\end{aligned}
$$

Applying the product rule for matrix derivatives in (4.93) to (4.105),

$$
\begin{aligned}
H\left[R_{0} ; \boldsymbol{\theta}\right] & =\left(\mathbf{e}_{1}^{\top} \otimes \mathbf{e}_{1}^{\top} \otimes \mathbf{I}_{s}\right) \frac{d}{d \boldsymbol{\theta}^{\top}} \operatorname{vec}\left[\left(\frac{d \mathrm{vec} \mathbf{R}}{d \boldsymbol{\theta}^{\top}}\right)^{\top}\right] \\
& =\left(\mathbf{e}_{1}^{\top} \otimes \mathbf{e}_{1}^{\top} \otimes \mathbf{I}_{s}\right) H[\operatorname{vec} \mathbf{R} ; \boldsymbol{\theta}]
\end{aligned}
$$

where $H[\operatorname{vecR} ; \boldsymbol{\theta}]$ as given by (4.95) (or 4.100 and 4.101 if appropriate).

\section{D Sensitivity of the stochastic growth rate}

As shown in Caswell (2001), Tuljapurkar's (1982) approximation for the stochastic growth rate can be written as

$$
\log \lambda_{s} \approx \log \lambda-\frac{\mathbf{D C D}^{\top}}{2 \lambda^{2}}
$$

where $\mathbf{C}$ is the symmetric covariance matrix for the population projection matrix and $\mathbf{D}$ is the Jacobian matrix (in this case, a row vector) for the dominant eigenvalue $\lambda$ of the mean population matrix $\overline{\mathbf{A}}$ :

$$
\begin{aligned}
\mathbf{D} & =D[\lambda ; \operatorname{vec} \overline{\mathbf{A}}] \\
& =\frac{\partial \lambda}{\partial \operatorname{vec}^{\top} \overline{\mathbf{A}}} .
\end{aligned}
$$

To find the sensitivity of the stochastic growth rate to $\overline{\mathbf{A}}$, differentiate (4.107) with respect to $\operatorname{vec}^{\top} \overline{\mathbf{A}}$ :

$$
\begin{aligned}
\frac{\partial \log \lambda_{s}}{\partial \operatorname{vec}^{\top} \overline{\mathbf{A}}} & =\frac{\mathbf{D}}{\lambda}+\frac{\mathbf{D}}{\lambda^{3}}\left(\mathbf{D C D}^{\top}\right)-\frac{1}{2 \lambda^{2}} \frac{\partial\left(\mathbf{D C D}^{\top}\right)}{\partial \operatorname{vec}^{\top} \overline{\mathbf{A}}} \\
& =\frac{\mathbf{D}}{\lambda}\left(1+\frac{\mathbf{D C} \mathbf{D}^{\top}}{\lambda^{2}}\right)-\frac{1}{2 \lambda^{2}} \frac{\partial\left(\mathbf{D C D}^{\top}\right)}{\partial \operatorname{vec}^{\top} \overline{\mathbf{A}}}
\end{aligned}
$$

To evaluate the derivative of $\mathbf{D C D}^{\top}$ with respect to $\overline{\mathbf{A}}$ in the second term, we will apply the product rule for vector derivatives (Magnus 2010). Given vectors $\mathbf{y}(n \times 1)$ and $\mathbf{x}(1 \times n)$, the 
derivative of their product with respect to a third vector $\mathbf{z}$ is

$$
\frac{d \operatorname{vec}(\mathbf{x y})}{d \operatorname{vec}^{\top} \mathbf{z}}=\mathbf{y}^{\top} \frac{d \operatorname{vec} \mathbf{x}}{d \operatorname{vec}^{\top} \mathbf{z}}+\mathbf{x} \frac{d \operatorname{vec} \mathbf{y}}{d \operatorname{vec}^{\top} \mathbf{z}} .
$$

Because $\mathbf{D C D}^{\top}$ is a scalar, vec $\left(\mathbf{D C D}^{\top}\right)=\mathbf{D C D}^{\top}$. Thus

$$
\frac{\partial\left(\mathbf{D C D}^{\top}\right)}{\partial \operatorname{vec}^{\top} \overline{\mathbf{A}}}=\mathbf{D C}^{\top} \frac{\partial \operatorname{vec} \mathbf{D}}{\partial \operatorname{vec}^{\top} \overline{\mathbf{A}}}+\mathbf{D C} \frac{\partial \operatorname{vec}\left(\mathbf{D}^{\top}\right)}{\partial \operatorname{vec}^{\top} \overline{\mathbf{A}}}
$$

Note that the Hessian of $\lambda$ with respect to $\overline{\mathbf{A}}$ is

$$
\begin{aligned}
H[\lambda ; \operatorname{vec} \overline{\mathbf{A}}] & =\frac{d}{d \operatorname{vec}^{\top} \overline{\mathbf{A}}} \operatorname{vec}\left[\left(\frac{d \lambda}{d \operatorname{vec}^{\top} \overline{\mathbf{A}}}\right)^{\top}\right] \\
& =\frac{\partial \operatorname{vec}\left(\mathbf{D}^{\top}\right)}{\partial \operatorname{vec}^{\top} \overline{\mathbf{A}}}=\frac{\partial \operatorname{vec}(\mathbf{D})}{\partial \operatorname{vec}^{\top} \overline{\mathbf{A}}} .
\end{aligned}
$$

Letting $\mathbf{H}=H[\lambda ; \operatorname{vec} \overline{\mathbf{A}}]$ and noting that $\mathbf{C}$ is a symmetric matrix, (4.111) simplifies to

$$
\begin{aligned}
\frac{\partial\left(\mathbf{D C D}^{\top}\right)}{\partial \operatorname{vec}^{\top} \overline{\mathbf{A}}} & =\mathbf{D C}^{\top} \mathbf{H}+\mathbf{D C H} \\
& =2 \mathbf{D C H}
\end{aligned}
$$

Substituting (4.113) into (4.109), we see that the sensitivity of the stochastic growth rate with respect to $\overline{\mathbf{A}}$ is

$$
\begin{aligned}
\frac{\partial \log \lambda_{s}}{\partial \operatorname{vec}^{\top} \overline{\mathbf{A}}} & =\frac{\mathbf{D}}{\lambda}\left(1+\frac{\mathbf{D} \mathbf{C D}^{\boldsymbol{\top}}}{\lambda^{2}}\right)-\frac{\mathbf{D C H}}{\lambda^{2}} \\
& =\frac{\mathbf{D}}{\lambda}\left(1-\frac{\mathbf{C H}}{\lambda}+\frac{\mathbf{D C} \mathbf{D}^{\boldsymbol{\top}}}{\lambda^{2}}\right) .
\end{aligned}
$$

\section{E Commutation matrix code}

The following MATLAB code calculates the commutation matrix $\mathbf{K}_{m, n}$ :

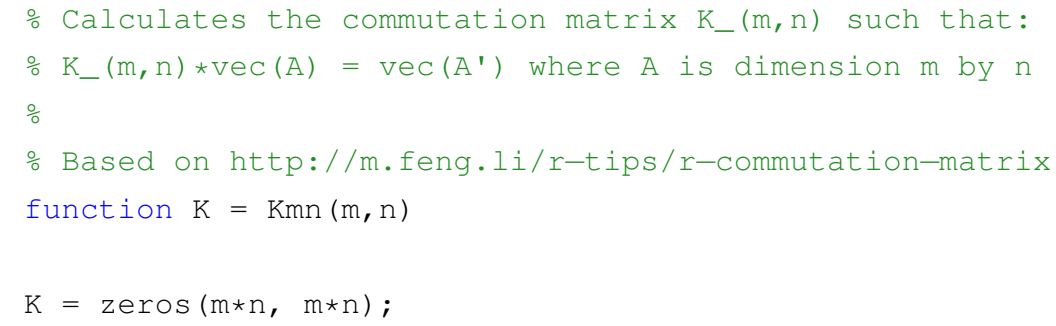




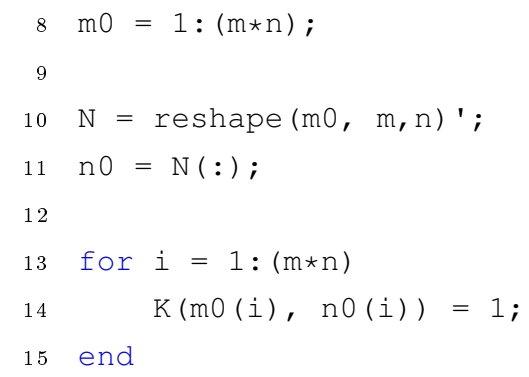




\section{Chapter 5}

\section{The evolution of facultative sex ratios with two maternal conditions}

\subsection{Abstract}

When the primary sex ratio (proportion of male offspring that a parent produces) varies with individual or environmental conditions, multiple sex ratios may coexist in the same population. Complex population structure may also result from having parents with different conditions. As a result, modeling the evolution of facultative (condition-dependent) sex ratios requires explicitly two-sex models with stage structure, and sex ratio strategies with multiple components.

We combine continuous-time matrix population models and multidimensional adaptive dynamics to analyze the evolution of sex ratios that are determined by one of two possible maternal conditions. We identify evolutionarily singular strategies for sex ratios that depend on maternal age or quality, and find many cases where one maternal condition produces exclusively male or female offspring. In particular, the sexes preferred by mothers of different qualities depend on relative reproductive value ratios for each sex.

Keywords: sex ratio evolution, facultative sex ratios, maternal condition, Trivers-Willard hypothesis, matrix population models, adaptive dynamics

\subsection{Introduction}

When the primary sex ratio (proportion of male births in an individual's offspring production strategy) is homogenous across the population, it evolves to equalize parental investment in both sexes (Fisher 1930). If males and females are equally costly, the sex ratio evolves to equality (Hamilton 1967). If males and females are differentially costly, the sex ratio skews in response to sex-specific offspring costs, such as differential offspring resource requirements, offspring mortality, or offspring-induced parental mortality (Shyu and Caswell xxb). 
However, many species have facultative (condition-dependent) sex ratio strategies, where a parent adjusts the primary sex ratio of its offspring depending on some environmental or individual condition (Leimar 1996, West 2009). The evolution of facultative sex ratio strategies is more complicated, because multiple sex ratios can coexist within the same population.

For example, different parents may use different sex ratios in response to spatial or temporal environmental variation. Parasitic wasps, which lay their eggs on a variety of hosts, vary their sex ratios based on host size (Charnov 1981); because female larvae benefit more greatly from larger food sources, wasp sex ratios are female-biased on large hosts and male-biased on small ones. Other species use different sex ratios in different seasons, in response to the timing of sex-specific life cycle events (Werren and Charnov 1978). Kestrels, for instance, shift their sex ratios over the breeding season to account for differences in male and female maturation (Pen et al. 1999).

Sex ratios may also vary with some parental (usually maternal) condition. In many mammals, where males have higher infant mortality rates (Trivers 1985), sex ratios become increasingly female-biased with maternal age. This may be because older mothers are more prone to death or sterility, and cannot replace lost sons as easily (Charlesworth 1977). Older fathers can also promote female-biased sex ratios; Drosophila melanogaster females with older mates tend to produce more female offspring, possibly because deleterious mutations in older fathers are more detrimental to sons (Long and Pischedda 2005). When older parents are more beneficial, sex ratio biases reverse. In Iberian red deer, for example, older females are larger, obtain more food, and expend more energy on reproduction. Sex ratios thus become increasingly male-biased with maternal age, because older mothers can afford the higher costs of sons (Landete-Castillejos 2004).

The effects of parental age are linked to the more general notion of parental quality. Higher quality parents may be larger, healthier, higher ranking, or have more resources for reproduction (Hewison and Caillard 1999). The Trivers-Willard hypothesis (Trivers and Willard 1973) predicts that a parent's quality affects the sex ratios of their offspring. If offspring quality is correlated with parental quality, low quality parents should preferentially produce offspring of the sex with higher reproductive success (e.g., number of offspring) at low quality, and vice versa for high quality parents.

Suppose that reproductive success of male offspring varies more steeply with quality than that of female offspring. Then low quality female offspring will have higher success than low quality male offspring, but this ranking will be reversed for high quality offspring (Figure 5-1). Thus low quality parents, doomed to produce low quality offspring, should favor females. High quality parents, anticipating the production of high quality offspring, should favor males. The influence of quality on reproductive success is usually described in terms of greater (males, in our example) or lesser (females) "variance in in reproductive success," and the Trivers-Willard hypothesis is usually phrased as a prediction that high quality mothers will invest more in the sex with greater variance in reproductive output. 


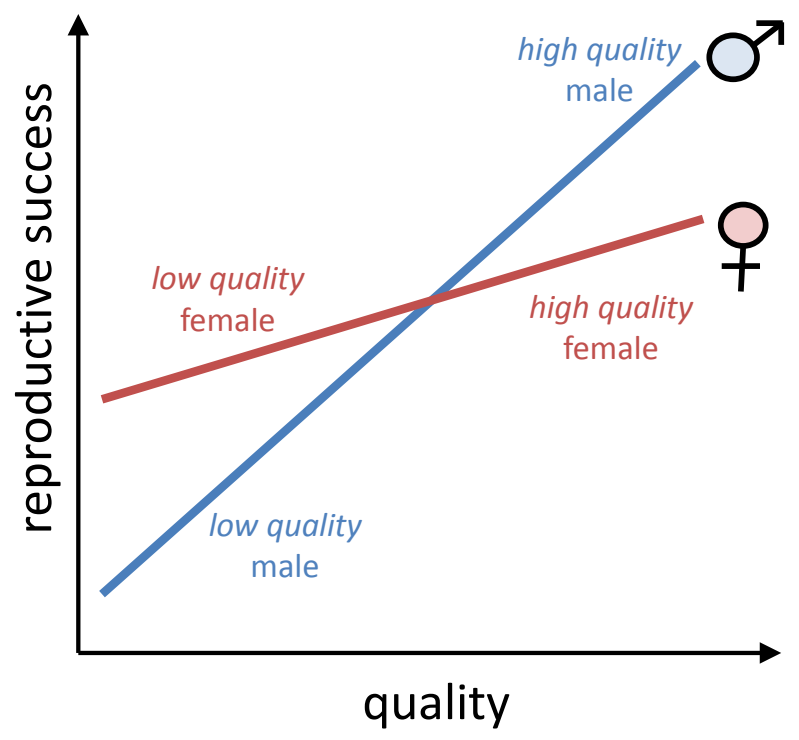

Figure 5-1: Reproductive success as a function of quality in the Trivers-Willard hypothesis. In this example, low quality females are more successful than low quality males, but high quality males and more successful than high quality females.

The example with males and females we used here is often applied to polygynous species, where high quality males monopolize most breeding opportunities. As a result, a high quality son outreproduces a high quality daughter, but a low quality daughter outreproduces a low quality son. Because males are the sex with the greater variance in reproductive success, high quality females adjust their sex ratios to invest in sons, while low-quality females invest in daughters.

Empirical support for the Trivers-Willard hypothesis has been found in many animals, most notably ungulates with strong sexual dimorphism and polygynous mating (e.g., Clutton-Brock et al 1984, 1986; Hewison and Gaillard 1999). However, results are sometimes mixed, possibly due to differences in data collection methods, quality measures, and difficulties in calculating lifetime reproductive success (Hewison and Gaillard 1999, Sheldon and West 2004). The effects of maternal quality on sex ratio have also been studied in birds (e.g., Kilner 1998, Clout et al. 2002), humans (e.g., Gaulin and Robbins 1991, Cameron and Dalerum 2009), and many other taxa (West 2009, Ch. 6)

Condition-dependent sex ratio shifts can confound conservation attempts, as when supplementary feeding of endangered kakapo parrots induced overly male-biased sex ratios (Clout et al. 2002). Alternatively, sex ratio shifts can be leveraged to promote the growth of small or vulnerable populations (Wedekind 2002). In every case, the complex population structures that arise from multiple maternal conditions may be challenging to incorporate into evolutionary projections.

To address this issue, we have developed a two-sex modeling framework that has multiple maternal states with different sex ratios. Our general model is introduced in Section 5.3.1, and further expanded upon in Sections 5.5.1 and 5.6.1. 
In our model, the facultative sex ratio strategy is described by the bivariate trait vector $\mathbf{s}$ :

$$
\mathbf{s}=\left(\begin{array}{l}
s_{1} \\
s_{2}
\end{array}\right)
$$

where $s_{1}$ is the sex ratio used by mothers in one condition (e.g., low quality) and $s_{2}$ is the sex ratio used by mothers in the other condition (e.g., high quality). Using multidimensional adaptive dynamics methods, as described in Section 5.3.3, we determine how $\mathbf{s}$ evolves over time and find its evolutionarily singular strategies $\mathbf{s}^{*}$, which are potential long-term evolutionary outcomes.

We then consider two specific cases where the sex ratio depends on maternal condition. In the first case (Section 5.5), young and old mothers can evolve different sex ratios. In the second case (Section 5.6), high and low quality mothers can evolve different sex ratios, as in the Trivers-Willard hypothesis.

\subsection{Model and methods}

Here, we present a two-sex population model that uses a series of matrices to describe various stages and life cycle processes (as in Shyu and Caswell xxa, xxb). Our model distinguishes between two individual conditions (e.g., age or quality) for both males and females. We accordingly incorporate four types of unions (male-female mated pairings), and different preferences for mating with partners in different conditions.

Each maternal condition produces offspring with a different sex ratio. We analyze the transient dynamics of bivariate sex ratio evolution using the canonical equation of adaptive dynamics, and the equilibrium dynamics by characterizing evolutionarily singular strategies (SSs) of the sex ratios.

\subsubsection{A two-sex matrix model with multiple maternal conditions}

Consider, as an example, a two-sex population consisting of Condition 1 and Condition 2 individuals (e.g., young and old individuals, low and high quality individuals, etc.). Males and females mate to form unions (here, monogamous couples) that produce new offspring.

Unions where the male partner is in Condition $i$ and the female partner is in Condition $j$ will be written as $u_{i j}$. As we shall see in Sections 5.5.1 and 5.6.1, the $u_{i j}$ may differ in available resources, fertilities, and other properties. We will specifically assume that any union with a Condition $j$ female has sex ratio $s_{j}$. This means that the primary sex ratio is a facultative trait that depends solely on maternal condition. 


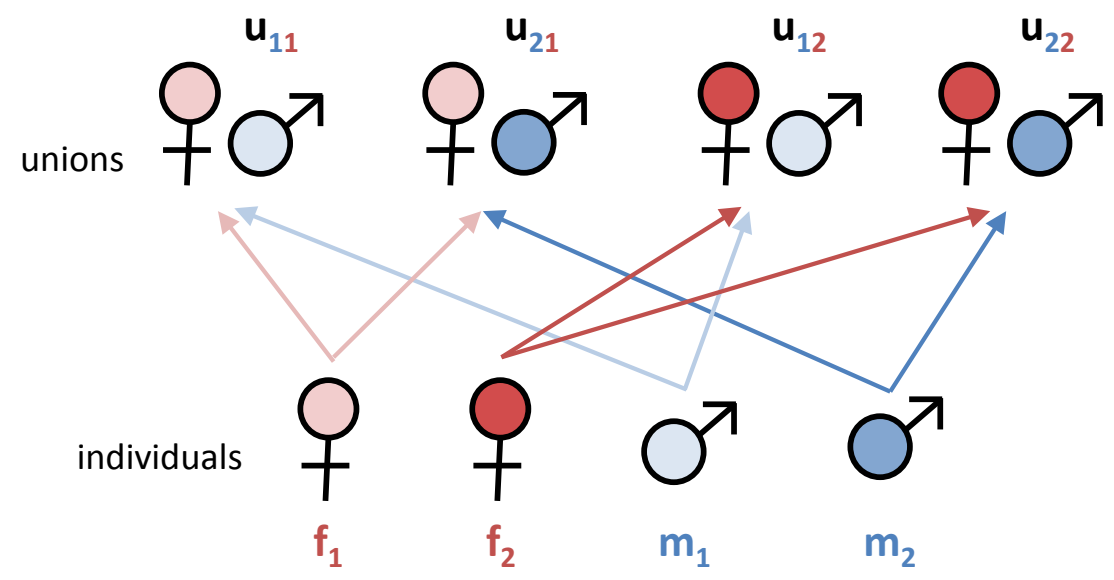

Figure 5-1: Stages of unmated individuals and mated unions in a two-sex population. Both males and females have two possible conditions.

The population consists of Condition 1 and 2 males $\left(m_{1}, m_{2}\right)$, Condition 1 and 2 females $\left(f_{1}, f_{2}\right)$, and four types of unions (Figure 5-1). The densities of each stage are given by the population vector:

$$
\mathbf{n}(t)=\left(\begin{array}{c}
m_{1} \\
m_{2} \\
f_{1} \\
f_{2} \\
u_{11} \\
u_{21} \\
u_{12} \\
u_{22}
\end{array}\right)=\left(\begin{array}{c}
\text { Condition } 1 \text { males } \\
\text { Condition } 2 \text { males } \\
\text { Condition } 1 \text { females } \\
\text { Condition } 2 \text { females } \\
\text { Condition } 1 \text { male }+ \text { Condition } 1 \text { female } \\
\text { Condition } 2 \text { male }+ \text { Condition } 1 \text { female } \\
\text { Condition } 1 \text { male }+ \text { Condition } 2 \text { female } \\
\text { Condition } 2 \text { male }+ \text { Condition } 2 \text { female }
\end{array}\right)
$$

Additional male, female, or union types can be added as new entries in the population vector.

We divide mating, birth, and life cycle transition processes into three rate matrices: $\mathbf{U}, \mathbf{B}$, and $\mathbf{T}$ respectively. As shown in Shyu and Caswell (xxa), the average of these matrices is the continuous-time projection matrix:

$$
\mathbf{A}(\mathbf{n})=\frac{1}{3}[\mathbf{T}+\mathbf{B}+\mathbf{U}]
$$

where

$$
\frac{d \mathbf{n}}{d t}=\mathbf{A}(\mathbf{n}) \mathbf{n}(t)
$$

Specific examples of both the population vector and rate matrices are given in Sections 5.5.1 and 5.6.1. As we shall see, each of the three rate matrices in (5.3) may depend on the population vector (5.2) or the sex ratio vector (5.1). 
In many two-sex models, including ours, the matrices in (5.3) depend on the relative frequencies, rather than absolute abundances, of males and females. These matrices are thus functions of the stage frequency vector:

$$
\mathbf{p}=\frac{\mathbf{n}}{\|\mathbf{n}\|}
$$

where $\|\mathbf{n}\|$ is the 1-norm of $\mathbf{n}$. As a result, (5.4) is a frequency-dependent model that converges to an equilibrium stage distribution $\hat{\mathbf{p}}$ and a growth rate $\lambda$ that is the dominant eigenvalue of $\mathbf{A}(\hat{\mathbf{p}})$. To calculate $\lambda$ and $\hat{\mathbf{p}}$, it is sufficient to consider the dynamics of $\mathbf{p}$ (Shyu and Caswell xxa):

$$
\frac{d \mathbf{p}}{d t}=\left(\mathbf{I}_{s}-\mathbf{p} \mathbf{1}^{\top}\right) \mathbf{A}(\mathbf{p}) \mathbf{p}
$$

To find $\hat{\mathbf{p}}$, we integrate (5.6) with the MATLAB ODE45 differential equation solver until $\mathbf{p}$ converges to $\hat{\mathbf{p}}$ (e.g., until vector entries do not change significantly over consecutive integration intervals). The population's long-term growth rate $\lambda$ is then the dominant eigenvalue of $\mathbf{A}(\hat{\mathbf{p}})$, which has corresponding right and left eigenvectors $\mathbf{w}$ and $\mathbf{v}$. Note that the right eigenvector is also the stable stage distribution; that is, $\mathbf{w}=\hat{\mathbf{p}}$.

\subsubsection{Mating preferences}

The mating process, where adult males and females pair into reproducing unions, is described by the union formation matrix $\mathbf{U}$. Mating functions in $\mathbf{U}$ give the rates of union formation as functions of the relative frequencies of males and females available to mate, and are thus functions of the stage frequency vector $\hat{\mathbf{p}}(5.5)$.

Mating preferences in the mating functions describe the probabilities of favoring partners of certain conditions. The female preference distribution $g_{j}(i)$ gives the proportion of Condition $j$ females that mate with Condition $i$ males. Similarly, the male preference distribution $h_{i}(j)$ gives the proportion of Condition $i$ males that mate with Condition $j$ females. Summing these distributions over all male and female conditions respectively yields a total probability of 1 :

$$
\begin{array}{ll}
\sum_{i} g_{j}(i)=1 \quad \forall j \\
\sum_{j} h_{i}(j)=1 \quad \forall i
\end{array}
$$

Examples of mating preference distributions include:

1. Fully assortative mating, where individuals only mate with partners in the same condition:

$$
\begin{aligned}
& g_{j}(i)=1 \text { if } i=j, 0 \text { else } \\
& h_{i}(j)=1 \text { if } i=j, 0 \text { else }
\end{aligned}
$$


2. Random mating, where individuals pick partners based on their relative abundances in the population:

$$
\begin{aligned}
& g_{j}(i)=\frac{m_{i}}{\sum_{i} m_{i}} \\
& h_{i}(j)=\frac{f_{j}}{\sum_{j} f_{j}}
\end{aligned}
$$

3. Biased mating, where individuals prefer partners of certain conditions. An attractiveness or competitiveness factor $c_{i}$ weighs the abundance of each partner condition, e.g.:

$$
\begin{aligned}
g_{j}(i) & =\frac{c_{i} m_{i}}{\sum_{i} c_{i} m_{i}} \\
h_{i}(j) & =\frac{c_{j} f_{j}}{\sum_{j} c_{j} f_{j}}
\end{aligned}
$$

Partners with larger $c_{i}$ are more preferable mates. If all $c_{i}$ are equal, (5.11) reduces to the random mating case (5.10). If $c_{i}=0$, individuals of stage $i$ do not mate.

The total mating function $M_{i j}(\mathbf{n})$ gives the total unions $u_{i j}$ (Condition $i$ males mated with Condition $j$ females) formed per time. Mating functions from the generalized weighted mean (Hölder mean) family, in particular, have the form:

$$
M_{i j}(\mathbf{n})=\left(b\left[f_{j} g_{j}(i)\right]^{a}+(1-b)\left[m_{i} h_{i}(j)\right]^{a}\right)^{\frac{1}{a}}
$$

where $0 \leq b \leq 1$ and $a<0$ (Hadeler 1989, Martcheva and Milner 2001). Note that $M_{i j}(\mathbf{n})$ is calculated only over individuals that are available to mate (i.e., adult single male stages $m_{i}$ and adult single female stages $f_{j}$ ). As a result, the mating function does not depend on the males and females in non-mating stages, such as immature juveniles or adults already in unions.

As in Shyu and Caswell (xxa, xxb), we will use a harmonic mean mating function where $a=$ $-1, b=1 / 2$, so that:

$$
M_{i j}(\mathbf{n})=\frac{2 m_{i} h_{i}(j) f_{j} g_{j}(i)}{m_{i} h_{i}(j)+f_{j} g_{j}(i)}
$$

The corresponding male and female per capita mating functions are:

$$
\begin{aligned}
U_{m, i j}(\mathbf{n}) & =\frac{M_{i j}(\mathbf{n})}{m_{i}} \\
U_{f, i j}(\mathbf{n}) & =\frac{M_{i j}(\mathbf{n})}{f_{j}}
\end{aligned}
$$

As we shall see, these per capita mating functions appear in the union matrix $\mathbf{U}$ in (5.4). 


\subsubsection{Multidimensional adaptive dynamics}

Adaptive dynamics is a phenotype-based framework for modeling evolution. We have previously used univariate (one-dimensional) adaptive dynamics to determine evolutionarily singular strategies for a single, scalar sex ratio (Shyu and Caswell xxb). Here, we use multidimensional adaptive dynamics to analyze the evolution of the bivariate sex ratio $\mathbf{s}$ in (5.1).

Similar to the approach in Shyu and Caswell (xxb), we consider a stable, monomorphic resident population with sex ratio phenotype $\mathbf{s}$, projection matrix $\mathbf{A}$ as in (5.3), and a long-term exponential growth rate $\lambda$ that is the dominant eigenvalue of $\mathbf{A}(\hat{\mathbf{p}})$. This resident population is invaded by new, rare mutants, which differ from residents only in terms of their sex ratio phenotype. Such mutations are small, rare, and infrequent. As a result, mutants do not affect resident dynamics, and will either die out or reach fixation before the next mutation arises (Geritz 1996, Metz 2006).

A given mutant has phenotype $\mathbf{s}^{\prime}$, projection matrix $\mathbf{A}^{\prime}$, and corresponding growth rate $\lambda^{\prime}$; both $\mathbf{A}^{\prime}$ and $\lambda^{\prime}$ depend on the environmental conditions (e.g., mating rates) set by the resident. The mutant projection matrix $\mathbf{A}^{\prime}$ is structurally identical to the resident matrix $\mathbf{A}$; however, $\mathbf{A}^{\prime}$ uses the mutant sex ratio $\mathbf{s}^{\prime}$ and is evaluated at the resident equilibrium stage distribution $\hat{\mathbf{p}}$. An example of how to construct $\mathbf{A}^{\prime}$ is shown in Shyu and Caswell (xxb, Section 3.2.1).

The invasion fitness $\Lambda_{\mathbf{s}}\left(\mathbf{s}^{\prime}\right)$ is the relative growth rate of a mutant with sex ratio strategy $\mathbf{s}^{\prime}$, in an environment where the resident uses the strategy $\mathbf{s}$ :

$$
\Lambda_{\mathbf{s}}\left(\mathbf{s}^{\prime}\right)=\lambda^{\prime}(\hat{\mathbf{p}})-\lambda
$$

Only mutants with a positive invasion fitness have a positive probability of displacing the resident.

The selection gradient is the first derivative of the invasion fitness (5.15) with respect to the mutant phenotype $\mathbf{s}^{\prime}$, and indicates the direction of selection at a resident phenotype $\mathbf{s}$. Note that the resident growth rate $\lambda$ does not depend on $\mathbf{s}^{\prime}$. Thus, the selection gradient is simply the sensitivity of mutant growth rate $\lambda^{\prime}$ (Caswell 2010):

$$
\begin{aligned}
\mathbf{D}(\mathbf{s}) & =\left.\frac{\partial \lambda^{\prime}}{\partial \mathbf{s}^{\prime \top}}\right|_{\mathbf{s}^{\prime}=\mathbf{s}} \\
& =\left.\left(\left(\mathbf{w}^{\prime \top} \otimes \mathbf{v}^{\prime \top}\right) \frac{d \mathrm{vec} \mathbf{A}^{\prime}}{d \mathbf{s}^{\prime \top}}\right)\right|_{\mathbf{s}^{\prime}=\mathbf{s}}
\end{aligned}
$$

where $\mathbf{w}^{\prime}$ and $\mathbf{v}^{\prime}$ are the dominant right and left eigenvectors of the mutant matrix $\mathbf{A}^{\prime}(\hat{\mathbf{p}})$, scaled so that $\mathbf{v}^{\prime \top} \mathbf{w}^{\prime}=1$.

Although the invasion fitness (5.15) is a scalar, the selection gradient (5.16) is a row vector with two components - the partial derivatives of $\lambda^{\prime}$ to each entry of $\mathbf{s}(5.1)$ :

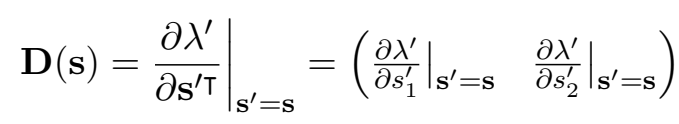


As shown in the next two sections, the selection gradient (5.17) lends insight into both the transient and equilibrium evolutionary dynamics of $\mathbf{s}$.

\subsubsection{Transient evolutionary dynamics}

The transient dynamics of $\mathbf{s}$ depend on the evolutionary trajectories generated by repeated mutant invasions. When mutations are small (do not differ drastically from the resident phenotype), these trajectories can be approximated by the canonical equation of adaptive dynamics.

As shown by Dieckmann and Law (1996) and Durinx et al. $(2005,2008)$, the canonical equation is a differential equation that describes $d \mathbf{s} / d t$, the change in the resident trait over time, using a first-order Taylor approximation. In both unstructured and structured populations, it can be written as the product of the selection gradient $\mathbf{D}(\mathbf{s})$ and a mutational variance-covariance matrix $\mathbf{V}(\mathbf{s})$ that encompasses mutation probabilities, frequencies, and effects (Doebeli 2011):

$$
\frac{d \mathbf{s}}{d t}=\mathbf{V}(\mathbf{s}) \mathbf{D}(\mathbf{s})
$$

The multivariate breeder's equation from quantitative genetics (Lande 1979) has a form similar to (5.18), but is based on standing genetic variation rather than the active mutational process (Doebeli 2011).

Though population size affects the mutation rate (Dieckmann and Law 1996), we will focus on the shape and direction of the evolutionary trajectories, rather than their speed, so that the population's (exponentially growing) size is irrelevant. We will also assume that effects of mutations on different components of $\mathbf{s}$ are uncorrelated (i.e., no pleiotropy), so that $\mathbf{V}(\mathbf{s})$ is a diagonal matrix.

The evolution of $\mathbf{s}$ is biologically constrained, in that neither $s_{1}$ nor $s_{2}$ can be less than 0 or greater than 1 (or both 0 and 1 simultaneously) in a realistic, viable population. These constraints can be written as:

$$
\begin{aligned}
& 0 \leq s_{1} \leq 1 \\
& 0 \leq s_{2} \leq 1 \\
& \left(s_{1}, s_{2}\right) \neq(0,0) \\
& \left(s_{1}, s_{2}\right) \neq(1,1)
\end{aligned}
$$

One can incorporate evolutionary constraints by adjusting the variance-covariance functions to restrict evolution in unfeasible directions (Dieckmann 2006). To this end, we use a mutational matrix of the form:

$$
\mathbf{V}(\mathbf{s})=\left(\begin{array}{cc}
s_{1}\left(1-s_{1}\right) & 0 \\
0 & s_{2}\left(1-s_{2}\right)
\end{array}\right)
$$


This choice of $\mathbf{V}$ causes the mutational variances to decrease as $s_{1}$ and $s_{2}$ near 0 or 1 . If either $s_{1}$ or $s_{2}$ goes to 0 or 1 , their corresponding component of the canonical equation (5.18) will vanish, preventing that sex ratio from evolving out of the biologically constrained region (5.19).

With mutational matrix (5.20) and selection gradient (5.17), the canonical equation (5.18) becomes:

$$
\begin{aligned}
\frac{d \mathbf{s}}{d t} & =\mathbf{V}(\mathbf{s}) \mathbf{D}^{\top}(\mathbf{s}) \\
& =\left(\begin{array}{cc}
s_{1}\left(1-s_{1}\right) & 0 \\
0 & s_{2}\left(1-s_{2}\right)
\end{array}\right)\left(\begin{array}{l}
\left.\frac{\partial \lambda^{\prime}}{\partial s_{1}}\right|_{\mathbf{s}^{\prime}=\mathbf{s}} \\
\left.\frac{\partial \lambda^{\prime}}{\partial s_{2}}\right|_{\mathbf{s}^{\prime}=\mathbf{s}}
\end{array}\right)
\end{aligned}
$$

We will use (5.21) to track the evolutionary trajectories of $\mathbf{s}$ through $2 \mathrm{D}$ trait space.

\subsubsection{Equilibrium evolutionary dynamics}

Potential evolutionary endpoints occur at stationary points of the canonical equation (5.21). The corresponding resident strategies $\mathbf{s}^{*}$ are called singular strategies (SSs), where:

$$
\left.\frac{d \mathbf{s}}{d t}\right|_{\mathbf{s}^{\prime}=\mathbf{s}=\mathbf{s}^{*}}=\left.\mathbf{V}(\mathbf{s}) \mathbf{D}(\mathbf{s})\right|_{\mathbf{s}^{\prime}=\mathbf{s}=\mathbf{s}^{*}}=0
$$

As summarized in Figure 5-2, there are five possible types of singular strategies. The most obvious type of singular strategy (Type 1, interior SS) occurs when both entries of the selection gradient $\mathbf{D}(\mathbf{s})$ (5.17) are simultaneously 0, indicating no directional selection on either component of $\mathbf{s}$ (Doebeli 2011). If there are no points in the biologically constrained region (5.19) where both entries of $\mathbf{D}(\mathbf{s})$ are 0 , there is no interior SS.

The remaining types of singular strategies (Types 2-5, boundary SSs) lie on each of the four boundaries of the constrained region, where $s_{1}$ or $s_{2}$ are either 0 or 1 (Leimar 1996, Schwanz 2006). In these cases, both components of $\mathbf{D}(\mathbf{s})$ do not simultaneously equal 0 . Instead, the selection gradient for the non-boundary sex ratio is 0 , and the selection gradient for the boundary sex ratio

points away from the boundary (Figure 5-2). Note that (5.22) can still be satisfied depending on the value of $\mathbf{V}(\mathbf{s})$.

In most cases, as shown in Schwanz (2006), there is a single SS $\mathbf{s}^{*}$, which falls into one of these five cases (but see Section 5.5.3 for an example where this is not true). To find $\mathbf{s}^{*}$, we use the following steps:

1. Determine if there is any point in the constrained region (5.19) where both components of the selection gradient (5.17) are simultaneously 0. This can be done using MATLAB's $f$ solve or fmincon functions. If a solution is found, this is an interior (Type 1) SS. Else, we must check for a boundary SSs of Types 2-5. 


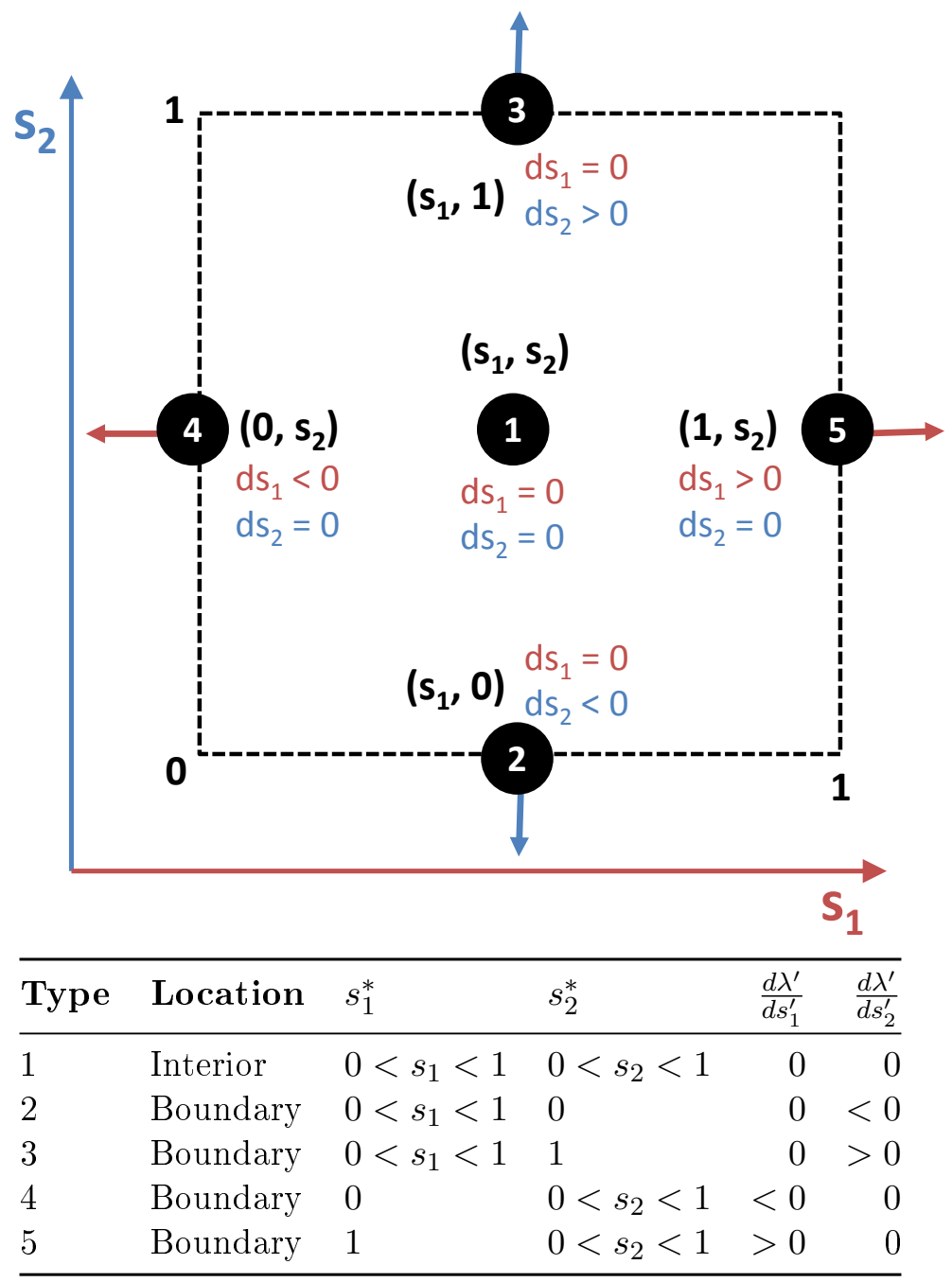

Figure 5-2: The five types of singular strategies $\mathbf{s}^{*}=\left(s_{1}^{*}, s_{2}^{*}\right)$ and their corresponding selection gradients $(5.17)$. Though it is also possible for $\mathbf{s}^{*}=(0,1)$ or $(1,0)$, these are marginal cases that we have not observed in our model. 
2. To check for a Type $2 \mathrm{SS}$, set $s_{2}=0$ and use MATLAB's fsolve function to find the corresponding value of $s_{1}$ where $\frac{d \lambda}{d s_{1}^{\prime}}=0$. If $\frac{d \lambda}{d s_{2}^{\prime}}<0$ at this point, it is $\mathbf{s}^{*}$.

3. To check for a Type $3 \mathrm{SS}$, set $s_{2}=1$ and find $s_{1}$ where $\frac{d \lambda}{d s_{1}^{\prime}}=0$. If $\frac{d \lambda}{d s_{2}^{\prime}}>0$, that point is $\mathbf{s}^{*}$.

4. To check for a Type $4 \mathrm{SS}$, set $s_{1}=0$ and find $s_{2}$ where $\frac{d \lambda}{d s_{2}^{\prime}}=0$. If $\frac{d \lambda}{d s_{1}^{\prime}}<0$, that point is $\mathbf{s}^{*}$.

5. To check for a Type $5 \mathrm{SS}$, set $s_{1}=1$ and find $s_{2}$ where $\frac{d \lambda}{d s_{2}^{\prime}}=0$. If $\frac{d \lambda}{d s_{1}^{\prime}}>0$, that point is $\mathbf{s}^{*}$.

These singular strategies $\mathbf{s}^{*}$ are potential evolutionary endpoints for $\mathbf{s}$. Characterizing their evolutionary and convergence stability can be challenging because $\mathbf{s}$ is a vector-valued trait (but see Appendix 5.B). Generating the evolutionary trajectories of $\mathbf{s}$ using (5.21), however, may lend insight into general stability patterns.

\subsection{Case studies}

We will examine two cases where the primary sex ratio depends on maternal condition. Again, the evolving sex ratio phenotype is the vector $\mathbf{s}=\left(s_{1}, s_{2}\right)$, the components of which are the sex ratios used by mothers in each condition.

The maternal conditions of interest are:

- Case 1: Maternal age. Young mothers have sex ratio $s_{1}$, old mothers have sex ratio $s_{2}$.

- Case 2: Maternal quality. Low quality mothers have sex ratio $s_{1}$, high quality mothers have sex ratio $s_{2}$.

In both cases, there are two possible conditions for an individual (young and old in Case 1; low and high quality in Case 2). Though individuals of different conditions may interbreed (e.g., a high quality male may mate with a low quality female), a couple's sex ratio is determined by the condition of the female partner (e.g., a couple with a low quality female would have sex ratio $s_{1}$ regardless of the male partner's quality).

In each case, we examine the evolutionary trajectories generated by the variance-constrained, bivariate canonical equation (5.21), and the types of evolutionarily singular strategies $\mathbf{s}^{*}$ (Figure 5-2) that result.

Unless otherwise indicated, model parameters are as in Table 5.1. Our model also makes the following assumptions:

- A union $u_{i j}$ (Condition $i$ male mated with Condition $j$ female) has divorce rate $d_{i j}$, reproductive rate $k_{j}$, and primary sex ratio $s_{j}$. Note that the reproductive rate and primary sex ratio are maternally determined.

- Only unions can produce new offspring. Unmated males and females do not reproduce independently. 
- Any offspring with a mutant parent also has the mutant phenotype; e.g., the mutant genotype is genetically dominant.

Results for all cases are summarized in Table 5.2.

\begin{tabular}{|c|c|c|}
\hline Parameter & Description & Value \\
\hline \multicolumn{3}{|c|}{ Both Cases } \\
\hline$C_{m}, C_{f}$ & $\begin{array}{l}\text { offspring resource costs (resources used per offspring } \\
\text { born) }\end{array}$ & $\begin{array}{l}\text { constants (Case } 2) \text { or given by }(5.27) \\
\text { (Case } 1 \text { b) }\end{array}$ \\
\hline$k_{j}$ & $\begin{array}{l}\text { reproductive rate (offspring born per time) of } \\
\text { Condition } j \text { mothers }\end{array}$ & $\begin{array}{l}\text { constants (Case } 1 \mathrm{a}) \text { or given by }(5.26) \\
\text { (Case } 1 \mathrm{~b} \text {, Case } 2)\end{array}$ \\
\hline$R_{j}$ & $\begin{array}{l}\text { resource investment rate (resources put into offspring } \\
\text { per time) of Condition } j \text { mothers }\end{array}$ & 10 \\
\hline$d_{i j}$ & divorce rate of union $u_{i j}$ & 0.1 \\
\hline$s_{j}$ & primary sex ratio of Condition $j$ mothers & component of $\mathbf{s}(5.1)$ \\
\hline$\mu_{m i}$ & male adult mortality rate in Condition $i$ & 0.1 \\
\hline & female adult mortality rate in Condition $j$ & 0.1 \\
\hline$U_{m, i j}$ & per capita mating rate of a male in union $u_{i j}$ & given by $(5.14)$ \\
\hline$\underline{U_{f, i j}}$ & per capita mating rate of a female in union $u_{i j}$ & given by $(5.14)$ \\
\hline \multicolumn{3}{|c|}{ Case 1 (maternal age) only } \\
\hline$\alpha_{m 1}, \alpha_{f 1}$ & juvenile to young adult maturation rates & 0.5 \\
\hline$\alpha_{m 2}, \alpha_{f 2}$ & young adult to old adult maturation rates & 0.5 \\
\hline$\mu_{m 0}, \mu_{f 0}$ & juvenile mortality rates & 0.1 \\
\hline & parental mortality intensity factor in (5.32) & 0.2 \\
\hline$I$ & baseline investment rate in $(5.27)$ & 1 \\
\hline \multicolumn{3}{|c|}{ Case 2 (maternal quality) only } \\
\hline$\alpha_{m 1}, \alpha_{f 1}$ & low quality juvenile to adult maturation rates & 1 \\
\hline$\alpha_{m 2}, \alpha_{f 2}$ & high quality juvenile to adult maturation rates & 1 \\
\hline$\mu_{m, 01}, \mu_{f, 01}$ & low quality juvenile mortality rates & 0.1 \\
\hline$\mu_{m, 02}, \mu_{f, 02}$ & high quality juvenile mortality rates & 0.1 \\
\hline$c_{i}$ & male competitiveness factor for Condition $i$ (5.40) & $c_{1}=0.1, c_{2}=0.9$ \\
\hline$q_{i j}$ & $\begin{array}{l}\text { probability that a Condition } j \text { female produces } \\
\text { Condition } i \text { offspring, subject to }(5.38)\end{array}$ & $q=q_{11}=q_{22}=0.65, q_{21}=q_{12}=1-q$ \\
\hline
\end{tabular}

Table 5.1: Two-sex model parameters. A subscript of $m$ indicates male, and a subscript of $f$ indicates female. In Case 1, Condition 1 individuals are young and Condition 2 individuals are old. In Case 2, Condition 1 individuals are low quality and Condition 2 individuals are high quality.

\subsection{Case 1: Maternal age}

Previous studies suggest that sex ratios differ with parental age when male and female offspring are differentially costly. However, different types of offspring costs may result in different bivariate sex ratio patterns.

Differential offspring costs can occur when offspring of one sex induce more parental mortality (Shyu and Caswell xxb, Case 4). Human sons, for instance, reduce maternal longevity more than daughters (Helle 2002). Younger mothers should thus favor daughters (the less mortality-inducing sex), while older mothers favor sons (the more mortality-inducing sex). Charnov (1982) suggested this was a potential example of senescence through antagonistic pleiotropy (Williams 1957), in that factors promoting a lower mortality reproductive strategy would be favored earlier in life, while a higher mortality strategy would be favored later in life. 


\begin{tabular}{|c|c|c|c|}
\hline Case & Offspring cost & Previous predictions & Model results \\
\hline \multicolumn{4}{|c|}{ Maternal age } \\
\hline Case 1a & $\begin{array}{l}\text { parental mortality } \\
\left(\text { Case } 4^{\dagger}\right)\end{array}$ & $\begin{array}{l}\text { young mothers favor sex inducing less } \\
\text { mortality, old mothers favor sex inducing } \\
\text { more mortality (Charnov 1982) }\end{array}$ & $\begin{array}{l}\text { results depend on relative reproductive } \\
\text { rates of young and old mothers (Figure } \\
5-3 \text { ) }\end{array}$ \\
\hline Case $1 \mathrm{~b}$ & $\begin{array}{l}\text { offspring mortality } \\
\text { during parental } \\
\text { investment (Case } 2^{\dagger} \text { ) }\end{array}$ & $\begin{array}{l}\text { young mothers favor higher mortality } \\
\text { sex, old mothers favor lower mortality } \\
\text { sex (Charlesworth 1977) }\end{array}$ & $\begin{array}{l}\text { infinitely many selectively neutral sex } \\
\text { ratio combinations (Figure 5-4) }\end{array}$ \\
\hline \multicolumn{4}{|c|}{ Maternal quality } \\
\hline Case 2 & $\begin{array}{l}\text { offspring resource cost } \\
\left(\text { Case } 1^{\dagger}\right)\end{array}$ & $\begin{array}{l}\text { high quality mothers favor the sex with } \\
\text { greater variance in reproductive success } \\
\text { or value (Trivers and Willard } 1973 \text {, } \\
\text { Leimar 1996) }\end{array}$ & $\begin{array}{l}\text { high quality mothers favor the sex with } \\
\text { greater variance in reproductive value at } \\
\text { boundary SS (Table 5.1) }\end{array}$ \\
\hline
\end{tabular}

Table 5.2: Evolutionarily singular strategies $\mathbf{s}^{*}$ for primary sex ratios that depend on maternal condition (age or quality). ${ }^{\dagger}$ Corresponding single sex ratio case in Shyu and Caswell (xxb).

Alternatively, differential offspring costs can occur when offspring of a particular sex are more likely to die before maturity (Shyu and Caswell xxb, Case 2). In many mammals, also including humans, male offspring have higher in utero mortality rates (Trivers 1985, Vatten 2004; though see also Orzack et al. 2015). Because older mothers are more likely to die or become sterile before they are able to replace lost sons, younger mothers should favor sons (the more mortality-prone sex), while older mothers favor daughters (the less mortality-prone sex) (Charlesworth 1977).

These two examples predict opposite trends for human sex ratios with maternal age. Empirical studies have alternatingly found sex ratios to increase (Takahashi 1954), decrease (Pollard 1969, James and Roston 1985), or be uncorrelated with maternal age (Almagor et al. 1998, Jacobsen et al. 1999). These mixed results may suggest that the effects of various offspring costs vary or even counterbalance in different populations, or that there are additional factors at play.

We will examine how the sex ratios of younger and older mothers are affected by sex-biased offspring costs. We consider both parental mortality and offspring mortality costs, in turn, through the following two subcases:

- Case 1a. Male and female offspring are differentially costly through their effects on parental mortality, similar to Charnov (1982). We previously described a similar single sex ratio model in Shyu and Caswell (xxb, Case 4).

- Case 1b. Male and female offspring have different mortality rates before maturity (during the period of parental investment), similar to Charlesworth (1977). We previously described a similar single sex ratio model in Shyu and Caswell (xxb, Case 2).

\subsubsection{Model}

We partition males and females into immature juveniles $\left(m_{0}, f_{0}\right)$, young adults $\left(m_{1}, f_{1}\right)$, and old adults $\left(m_{2}, f_{2}\right)$. Only young and old adults can mate to form reproducing unions, and the four 
possible union types are:

$$
\begin{aligned}
& u_{11}=\text { union of } m_{1} \text { and } f_{1} \\
& u_{21}=\text { union of } m_{2} \text { and } f_{1} \\
& u_{12}=\text { union of } m_{1} \text { and } f_{2} \\
& u_{22}=\text { union of } m_{2} \text { and } f_{2}
\end{aligned}
$$

The population vector (5.2) has 10 stages total:

$$
\mathbf{n}(t)=\left(\begin{array}{llllllllll}
m_{0} & m_{1} & m_{2} & f_{0} & f_{1} & f_{2} & u_{11} & u_{21} & u_{12} & u_{22}
\end{array}\right)^{\top}
$$

We will write a model of the form (5.4), and the next three sections give the matrices $\mathbf{B}, \mathbf{U}$, and $\mathbf{T}$ in turn.

\subsubsection{Births (B)}

Unions with young adult and old adult females use the sex ratios $s_{1}$ and $s_{2}$ respectively, and have characteristic reproductive rates $k_{1}$ and $k_{2}$ respectively. The birth matrix $\mathbf{B}$ is thus:

$$
\mathbf{B}=\left(\begin{array}{cccccccccc}
0 & 0 & 0 & 0 & 0 & 0 & s_{1} k_{1} & s_{1} k_{1} & s_{2} k_{2} & s_{2} k_{2} \\
0 & 0 & 0 & 0 & 0 & 0 & 0 & 0 & 0 & 0 \\
0 & 0 & 0 & 0 & 0 & 0 & 0 & 0 & 0 & 0 \\
0 & 0 & 0 & 0 & 0 & 0 & \left(1-s_{1}\right) k_{1} & \left(1-s_{1}\right) k_{1} & \left(1-s_{2}\right) k_{2} & \left(1-s_{2}\right) k_{2} \\
0 & 0 & 0 & 0 & 0 & 0 & 0 & 0 & 0 & 0 \\
0 & 0 & 0 & 0 & 0 & 0 & 0 & 0 & 0 & 0 \\
0 & 0 & 0 & 0 & 0 & 0 & 0 & 0 & 0 & 0 \\
0 & 0 & 0 & 0 & 0 & 0 & 0 & 0 & 0 & 0 \\
0 & 0 & 0 & 0 & 0 & 0 & 0 & 0 & 0 & 0 \\
0 & 0 & 0 & 0 & 0 & 0 & 0 & 0 & 0 & 0
\end{array}\right)
$$

In Case 1a, where male and female offspring have different effects on parental mortality, the $k_{j}$ are fixed rates. In Case $1 \mathrm{~b}$, where male and female offspring have different mortality rates during the period of parental investment, $k_{j}$ becomes:

$$
k_{j}=\frac{R_{j}}{s_{j} C_{m}+\left(1-s_{j}\right) C_{f}}
$$

where $R_{j}$ is the mother's rate of resource investment (total resources put into offspring per time), and $C_{m}$ and $C_{f}$ are the average male and female offspring resource costs (resources consumed per 
offspring born). In Shyu and Caswell (xxb, Case 2), these costs are shown to be:

$$
\begin{aligned}
C_{m} & =\frac{I}{\mu_{m 0}}\left(1-e^{\frac{-\mu_{m 0}}{\alpha_{m 1}}}\right) \\
C_{f} & =\frac{I}{\mu_{f 0}}\left(1-e^{\frac{-\mu_{f 0}}{\alpha_{f 1}}}\right)
\end{aligned}
$$

where $I$ is a constant baseline investment rate, $\alpha_{m 1}$ and $\alpha_{f 1}$ are the male and female juvenile to adult maturation rates, and $\mu_{m 0}$ and $\mu_{f 0}$ are the male and female juvenile mortality rates.

\subsubsection{Union formation (U)}

The union formation matrix $\mathbf{U}$ contains per capita mating rates of each union type. Using a harmonic mean mating function as in (5.13), the per capita mating functions (5.14) are:

$$
\begin{aligned}
U_{m 11} & =U_{m 21}=\frac{2 f_{1}}{m+f} \\
U_{m 12} & =U_{m 22}=\frac{2 f_{2}}{m+f} \\
U_{f 11} & =U_{f 12}=\frac{2 m_{1}}{m+f} \\
U_{f 21} & =U_{f 22}=\frac{2 m_{2}}{m+f}
\end{aligned}
$$

where $m=m_{1}+m_{2}$ and $f=f_{1}+f_{2}$.

The matrix $\mathbf{U}$ is then:

$$
\mathbf{U}=\left(\begin{array}{cccccccccc}
0 & 0 & 0 & 0 & 0 & 0 & 0 & 0 & 0 & 0 \\
0 & -\left(U_{m 11}+U_{m 12}\right) & 0 & 0 & 0 & 0 & 0 & 0 & 0 & 0 \\
0 & 0 & -\left(U_{m 21}+U_{m 22}\right) & 0 & 0 & 0 & 0 & 0 & 0 & 0 \\
0 & 0 & 0 & 0 & 0 & 0 & 0 & 0 & 0 & 0 \\
0 & 0 & 0 & 0 & -\left(U_{f 11}+U_{f 21}\right) & 0 & 0 & 0 & 0 & 0 \\
0 & 0 & 0 & 0 & 0 & -\left(U_{f 12}+U_{f 22}\right) & 0 & 0 & 0 & 0 \\
0 & \frac{1}{2} U_{m 11} & 0 & 0 & \frac{1}{2} U_{f 11} & 0 & 0 & 0 & 0 & 0 \\
0 & 0 & \frac{1}{2} U_{m 21} & 0 & \frac{1}{2} U_{f 21} & 0 & 0 & 0 & 0 & 0 \\
0 & \frac{1}{2} U_{m 12} & 0 & 0 & 0 & \frac{1}{2} U_{f 12} & 0 & 0 & 0 & 0 \\
0 & 0 & \frac{1}{2} U_{m 22} & 0 & 0 & \frac{1}{2} U_{f 22} & 0 & 0 & 0 & 0
\end{array}\right)
$$

\subsubsection{Transitions (T)}

Each stage has a characteristic mortality rate:

$$
\mu_{x s} \text { where } x \in\{m, f\} \text { and } s \in\{0,1,2\}
$$

If offspring impose parental mortality (Case 1a), the mortality rates of individuals in reproducing unions is greater than that of unmated individuals. Let $\mu_{x s}(5.30)$ be the mortality rate of an unmated individual, and $\mu_{x s}^{i j}$ be the mortality rate of a mated individual in union $u_{i j}$. Similar to 
Shyu and Caswell (xxb, Case 4), $\mu_{x s}^{i j}$ is increased from $\mu_{x s}$ by an amount $\gamma_{j}$ :

$$
\mu_{x s}^{i j}=\mu_{x s}+\gamma_{j}
$$

Let $\beta$ be a nonnegative constant that modulates the intensity of offspring-induced mortality. In Case $1 \mathrm{a}, \beta$ is a positive constant. In Case $1 \mathrm{~b}$, offspring do not affect parental mortality, so $\beta$ is 0 . Then $\gamma_{j}$ can be written as:

$$
\begin{aligned}
\gamma_{1} & =\beta k_{1}\left[s_{1} C_{m}+\left(1-s_{1}\right) C_{f}\right] \\
\gamma_{2} & =\beta k_{2}\left[s_{2} C_{m}+\left(1-s_{2}\right) C_{f}\right]
\end{aligned}
$$

Again, the average offspring costs $C_{m}$ and $C_{f}$ are given by (5.27).

Juveniles mature into young adults at a rate $\alpha_{m 1}$ for males and $\alpha_{f 1}$ for females, young adults mature into old adults at a rate $\alpha_{m 2}$ for males and $\alpha_{f 2}$ for females, and old adults cannot transition into any other prior stage. It is possible for a couple of one type to transition into another if a partner matures (e.g., a $u_{11}$ union will become a $u_{12}$ union if the young female partner matures into an old female). Unions may also divorce at a rate $d_{i j}$ or dissolve due to partner death, with mortality rates given by (5.31). The full transition matrix is $\mathbf{T}=\left[\mathbf{T}_{1} \mid \mathbf{T}_{2}\right]$ where:

$$
\begin{aligned}
& \mathbf{T}_{\mathbf{1}}=\left(\begin{array}{cccccc}
-\left(\mu_{m 0}+\alpha_{m 1}\right) & 0 & 0 & 0 & 0 & 0 \\
\alpha_{m 1} & -\left(\mu_{m 1}+\alpha_{m 2}\right) & 0 & 0 & 0 & 0 \\
0 & \alpha_{m 2} & -\mu_{m 2} & 0 & 0 & 0 \\
0 & 0 & 0 & -\left(\mu_{f 0}+\alpha_{f 1}\right) & 0 & 0 \\
0 & 0 & 0 & \alpha_{f 1} & -\left(\mu_{f 1}+\alpha_{f 2}\right) & 0 \\
0 & 0 & 0 & 0 & \alpha_{f 2} & -\mu_{f 2} \\
0 & 0 & 0 & 0 & 0 & 0 \\
0 & 0 & 0 & 0 & 0 & 0 \\
0 & 0 & 0 & 0 & 0 & 0 \\
0 & 0 & 0 & 0 & 0 & 0
\end{array}\right) \\
& \mathbf{T}_{\mathbf{2}}=\left(\begin{array}{cccc}
0 & 0 & 0 & 0 \\
\left(\mu_{f 1}+\gamma_{11}+d_{11}\right) & 0 & \left(\mu_{f 2}+\gamma_{12}+d_{12}\right) & 0 \\
0 & \left(\mu_{f 1}+\gamma_{21}+d_{21}\right) & 0 & \left(\mu_{f 2}+\gamma_{22}+d_{22}\right) \\
0 & 0 & 0 & 0 \\
\left(\mu_{m 1}+\gamma_{11}+d_{11}\right) & \left(\mu_{m 2}+\gamma_{21}+d_{21}\right) & 0 & 0 \\
0 & 0 & \left(\mu_{m 1}+\gamma_{12}+d_{12}\right) & \left(\mu_{m 2}+\gamma_{22}+d_{22}\right) \\
-\left(\mu_{m 1}+\mu_{f 1}+2 \gamma_{11}+\right. & 0 & 0 & 0 \\
\left.d_{11}+\alpha_{m 2}+\alpha_{f 2}\right) & -\left(\mu_{m 2}+\mu_{f 1}+2 \gamma_{21}+\right. & 0 & 0 \\
\alpha_{m 2} & \left.d_{21}+\alpha_{f 2}\right) & -\left(\mu_{m 1}+\mu_{f 2}+\gamma_{12}+\right. & 0 \\
\alpha_{f 2} & 0 & \left.d_{12}+\alpha_{m 2}\right) & -\left(\mu_{m 2}+\mu_{f 2}+2 \gamma_{22}+\right. \\
0 & \alpha_{f 2} & \alpha_{m 2} & \left.d_{22}\right)
\end{array}\right)
\end{aligned}
$$

\subsubsection{Results (Case 1a: Parental mortality cost)}

Suppose that male and female offspring have different costs on parental mortality. As an example, let female offspring be somewhat more costly $\left(C_{f}=0.6, C_{m}=0.4\right.$ in (5.32)). As a baseline case, consider the scenario where young and old mothers have the same reproductive rate $k_{1}=k_{2}$. In this 
case, all mothers have the same vital rates and reproductive abilities, regardless of their age. Figure 5 -1a shows the direction and relative magnitudes of the selection gradients (blue), as functions of the age-specific sex ratios $s_{1}$ and $s_{2}$.

Note that the evolutionary trajectories of $\mathbf{s}$ (red) converge not to one singular strategy, but instead to a whole line of strategies. Changing the offspring costs $C_{m}$ and $C_{f}$ yields qualitatively similar behavior (results not shown). Along this line of strategies, both components of the selection gradient are 0 , indicating the absence of selective pressure. Thus, if $\mathbf{s}$ is initialized at different values of $s_{1}$ and $s_{2}$, its corresponding evolutionary endpoints may differ significantly. However, the same average primary sex ratio:

$$
\bar{s}=\frac{s_{1}\left(u_{11}+u_{21}\right)+s_{2}\left(u_{12}+u_{22}\right)}{\sum u_{i j}}
$$

is shared by all the trajectory endpoints, where $\bar{s} \approx 0.6$ (Figure $5-1 b$ ). This is the same value expected from the equal investment principle in the single sex ratio case (Shyu and Caswell xxb), where the optimal single sex ratio $s^{*}$ evolves to:

$$
s^{*}=\frac{C_{f}}{C_{m}+C_{f}}
$$

Ultimately, it appears that any combination of $s_{1}$ and $s_{2}$ that leads to $\bar{s} \approx 0.6$ is a selectively neutral point on a line of singular strategies. Presumably because young and old females have similar reproductive rates, male and female offspring production can be partitioned between them in an infinite number of ways.

Now consider the case where the reproductive rate $k_{j}$ changes with age. When younger and older mothers are sufficiently different, the line of selectively neutral strategies disappears, and $\mathbf{s}$ converges to a single endpoint $\mathbf{s}^{*}$ regardless of its initial condition (Figure 5-2). The methods in Section 5.3.3.2 identify these endpoints as boundary SSs. If the reproductive rate increases with age $\left(k_{1}<k_{2}\right.$, Figure 5-2a), $s_{1}$ evolves to 0 (Type 4 SS), meaning that younger mothers are producing only the more costly females. If the reproductive rate decreases with age $\left(k_{1}>k_{2}\right.$, Figure $\left.5-2 \mathrm{~b}\right), s_{1}$ evolves to 1 (Type $5 \mathrm{SS}$ ), so that younger mothers are producing only the less costly males.

Figure 5-3 shows $\mathbf{s}^{*}$ for a range of offspring costs on parental mortality. If the reproductive rate increases with age (Figure 5-3a), older fertile females avoid the costly sex, while younger, less fertile females compensate by producing only the costly sex. When $C_{m}<C_{f}$, for example, young mothers produce only the more costly females $\left(s_{1}=0\right)$; when $C_{m}>C_{f}$, they switch to producing only the more costly males $\left(s_{1}=1\right)$. The older sex ratio $s_{2}$ favors the less mortality-inducing sex but, unlike $s_{1}$, never evolves to exclusively producing a single sex. When costs become increasingly unequal $\left(C_{m}>>C_{f}\right.$ or $\left.C_{m}<<C_{f}\right), s_{2}$ diverges more from $s_{1}$.

If the reproductive rate decreases with age (Figure 5-3b), the directions of the sex ratio biases reverse. Younger, more fertile females produce only the cheaper sex $\left(s_{1}=0\right.$ or $\left.s_{1}=1\right)$, forcing older, less fertile females to produce the costlier sex. When costs become increasingly unequal $\left(C_{m} \rightarrow 0\right.$ or $C_{m} \rightarrow 1$ ), we see that $s_{2}$ diverges less from $s_{1}$. Older mothers can produce more of the costlier 
a)

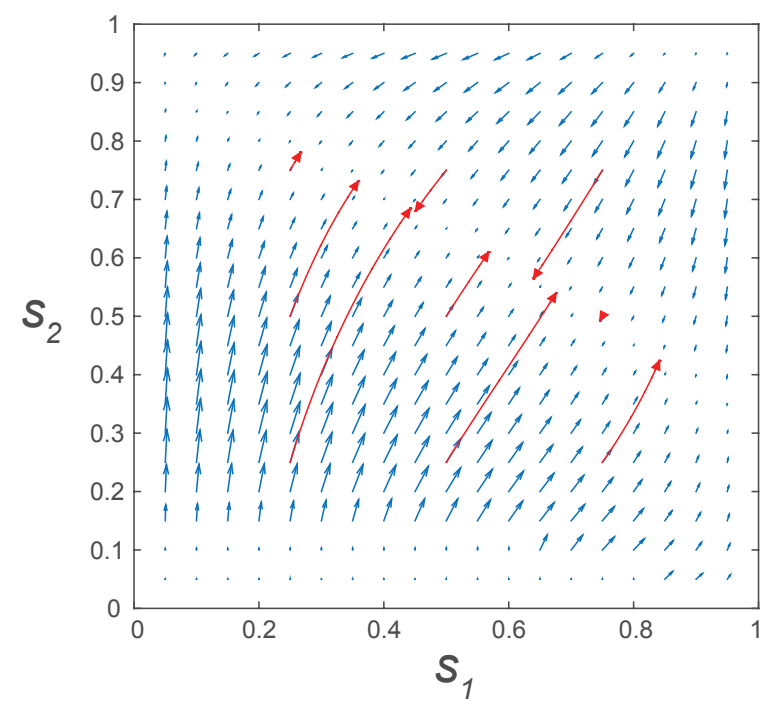

b)

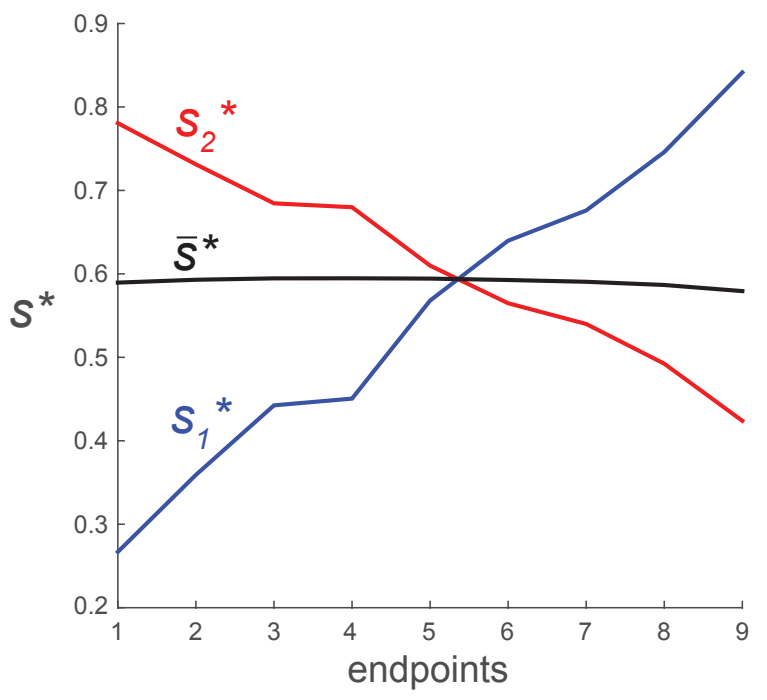

Figure 5-1: Evolutionary trajectories for Case 1a when all mothers have the same vital and reproductive rates $\left(k_{1}=k_{2}=10\right)$. Offspring costs $C_{m}=0.4, C_{f}=0.6$; other model parameters are as in Table 5.1. a) Selection gradients as functions of the age-specific sex ratios $s_{1}$ (younger females) and $s_{2}$ (older females). Blue arrows indicate the directions and relative magnitudes of the selection gradient (5.16). Red arrows indicate the evolutionary trajectories of $\mathbf{s}$ given by the canonical equation (5.21). b) The young, old, and average primary sex ratios $s_{1}, s_{2}$, and $\bar{s}(5.34)$ at the trajectory endpoints in Figure 5-1a.

a) older mothers more fertile

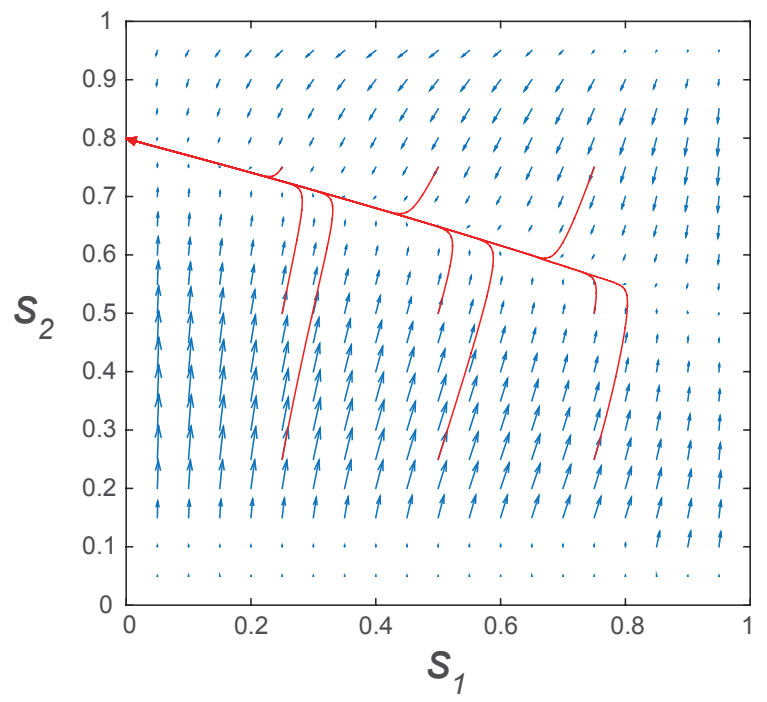

b) younger mothers more fertile

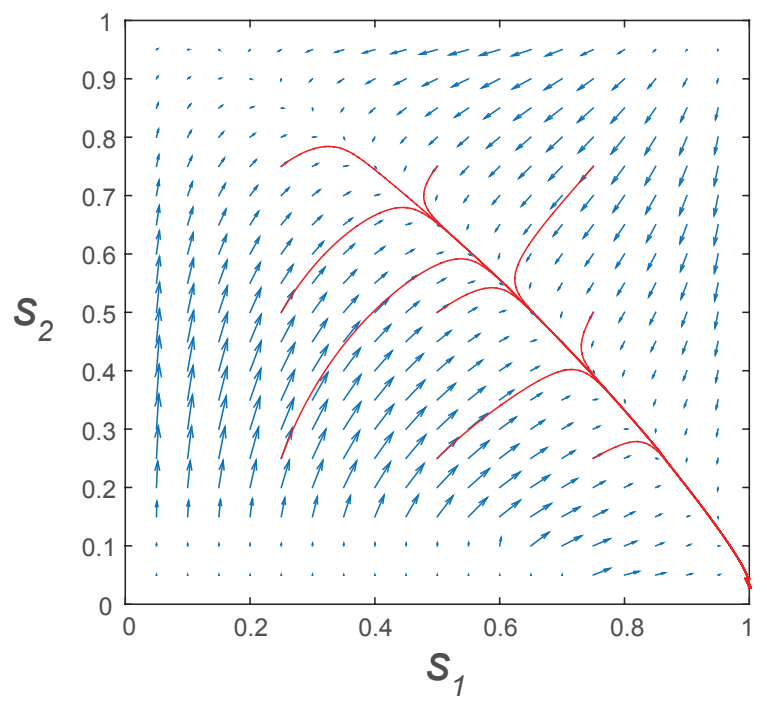

Figure 5-2: Evolutionary trajectories for Case 1a when young and old mothers have different reproductive rates. Offspring costs $C_{m}=0.4, C_{f}=0.6$; other model parameters are as in Table 5.1. a) Trajectories when older mothers are more fertile $\left(k_{1}=5, k_{2}=15\right)$. b) Trajectories when younger mothers are more fertile $\left(k_{1}=15, k_{2}=5\right)$. 
sex when the sex-specific costs are similar $\left(C_{m} \approx C_{f}\right)$, but avoid it when cost differences are high $\left(C_{m}>>C_{f}\right.$ or $\left.C_{m}<<C_{f}\right)$. This contrasts with how younger females produce only the costlier sex in Figure 5-3a.

a) older mothers more fertile

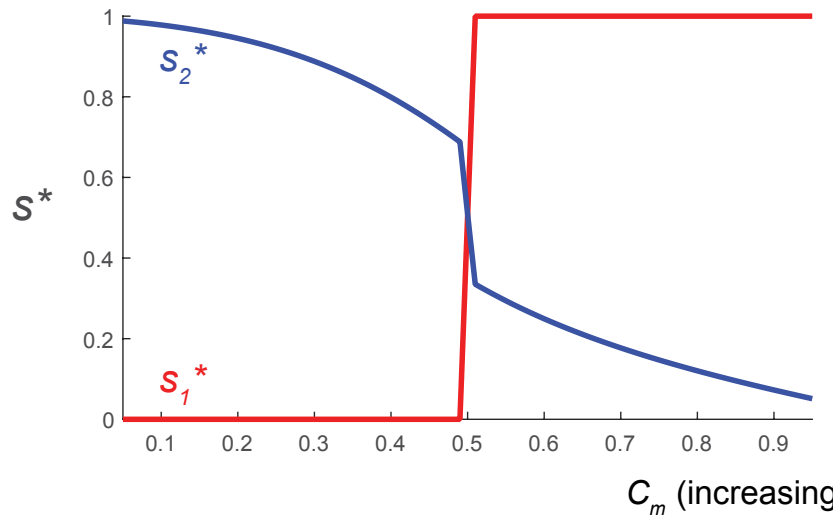

b) younger mothers more fertile

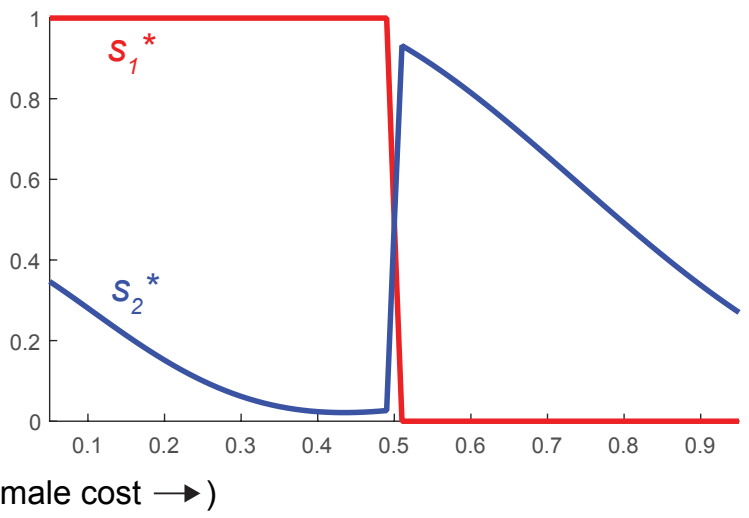

Figure 5-3: Sex ratio singular strategies $\mathbf{s}^{*}$ ( $s_{1}^{*}$ for younger females, $s_{2}^{*}$ for older females) as a function of the male offspring $\operatorname{cost} C_{m}$, which affects parental mortality via (5.32). The female offspring cost $C_{f}=1-C_{m}$ (more costly males mean less costly females); other model parameters are as given in Table 5.1. a) Values of $\mathbf{s}^{*}$ when older mothers are more fertile $\left(k_{1}=5, k_{2}=15\right)$. b) Values of $\mathbf{s}^{*}$ when younger mothers are more fertile $\left(k_{1}=15, k_{2}=5\right)$.

\subsubsection{Results (Case 1b: Parental resource cost)}

Suppose that male and female offspring do not affect parental mortality, but experience different mortality rates during the period of parental investment. As in Case 1a with identical mothers (Figure 5-1), s ultimately converges to a selectively neutral line of singular strategies (Figure 5-4). Unlike Case $1 \mathrm{a}$, this line persists even when young and old females differ in reproductive rates $k_{j}$ or baseline mortality rates $\mu_{f j}$. Once again, all combinations of $s_{1}$ and $s_{2}$ on the line share a similar average primary sex ratio $\overline{\mathbf{s}}$ (Figure 5 -4, right column).

As a result, the population may converge to any one of an infinite number of sex ratio combinations, which are selectively neutral and have same average primary sex ratio. The sex ratios observed in the long-term may accordingly vary with the initial state of $\mathbf{s}$.

\subsection{Case 2: Maternal quality}

As described in Section 5.2, the Trivers-Willard hypothesis (Trivers and Willard 1973) predicts that the primary sex ratio produced by a mother should depend on maternal quality. Specifically, high quality females will preferentially invest in the sex whose reproductive success varies most with quality. This hypothesis has three main assumptions: 
a)

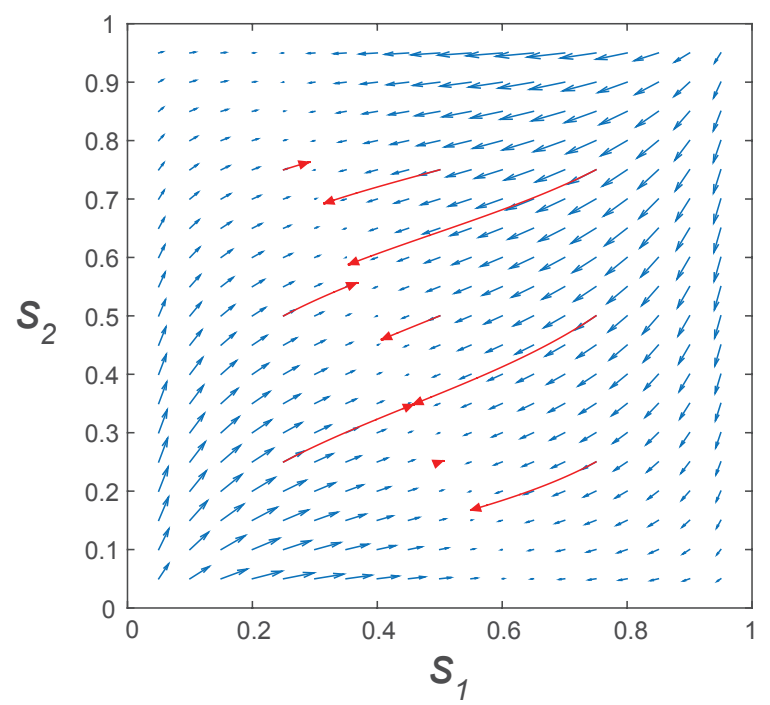

c)

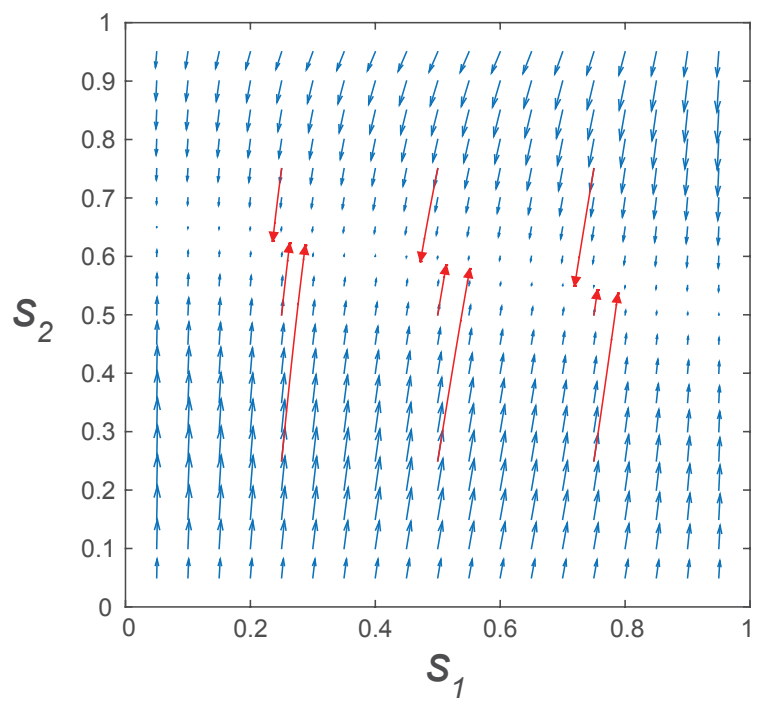

b)

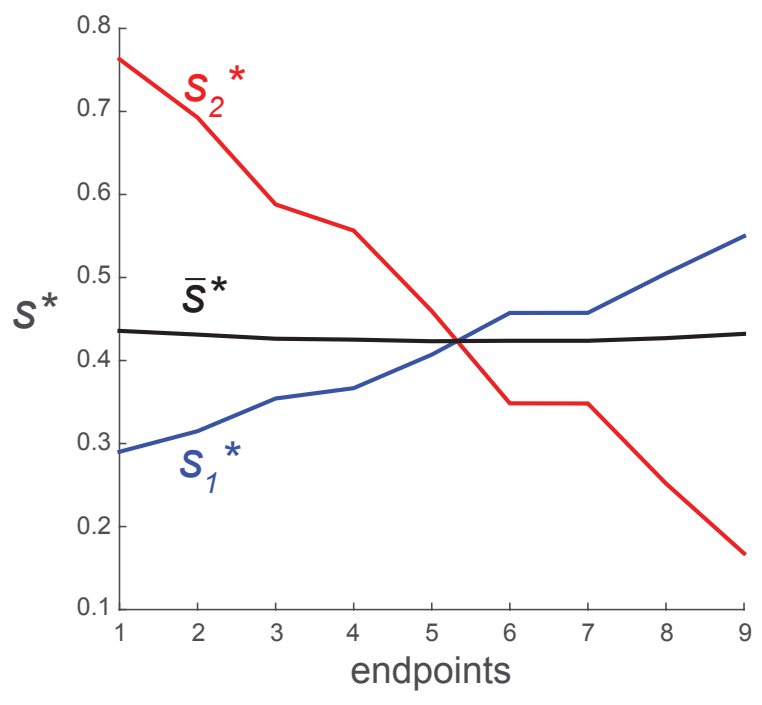

d)

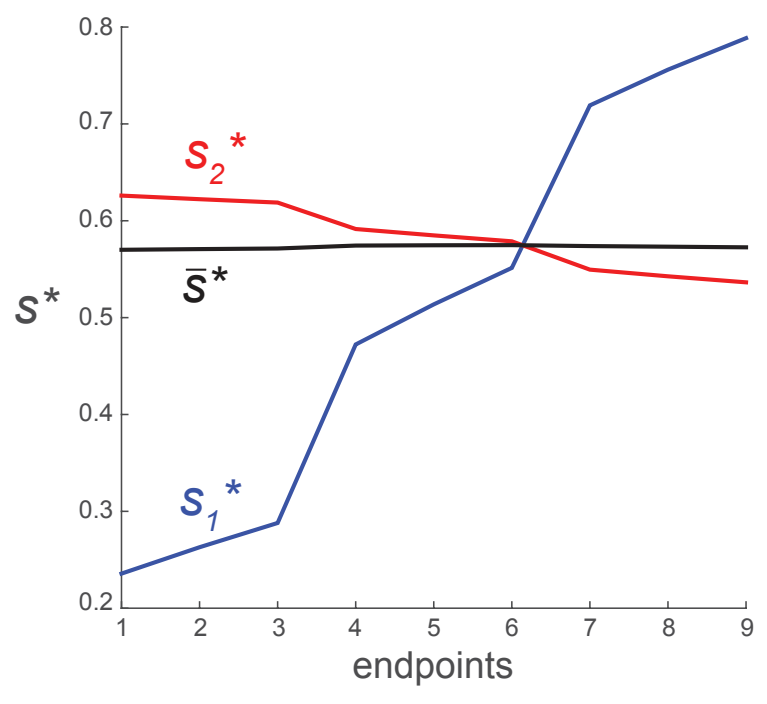

Figure 5-4: Example evolutionary trajectories and the sex ratios ( $s_{1}$ for younger females, $s_{2}$ for older females) at their endpoints for Case 1b. a) Trajectories for $C_{m}=0.2, C_{f}=0.8, \mu_{f 1}=0.1, \mu_{f 2}=$ $0.5, k_{1}=15, k_{2}=5$. b) Trajectories for $C_{m}=0.8, C_{f}=0.2, \mu_{f 1}=0.5, \mu_{f 2}=0.1, k_{1}=5, k_{2}=15$. Other model parameters are as given in Table 5.1. 
1. An offspring's quality carries into adulthood. In comparison to their low quality counterparts, high quality offspring will be larger, stronger, or have higher social ranks throughout their lifetimes. These advantages ultimately confer greater reproductive success or higher reproductive value (Leimar 1996).

We will specifically consider two main advantages that high quality adults have over low quality adults. These advantages concern the male competitive factor $c_{i}$ and female resource investment rate $R_{j}$ described in Sections 5.6.1.2 and 5.6.1.1 respectively. We will assume that one or both of the following advantages is present.

- High quality males obtain a greater proportion of total matings, and thus have a greater competiveness factor $\left(c_{2}>c_{1}\right)$.

- High quality females invest more resources into offspring production, and thus have a greater resource investment rate $\left(R_{2}>R_{1}\right)$.

2. The quality of an offspring is correlated with the quality of its parent (usually the mother). As shown in Section 5.6.1.1, we incorporate maternal quality transmission via a quality inheritance probability $q_{i j}$. High quality females will be more likely to produce high quality offspring, while low quality females will be more likely to produce low quality offspring.

Maternal transmission of quality occurs in many species, especially those with small broods (Trivers and Willard 1973); high ranking red deer mothers, for instance, produce larger and more dominant offspring (Clutton-Block et al. 1986). Quality transmission also affects the value of female offspring; when offspring quality depends mostly on maternal quality, high quality females are more productive in the long run (Leimar 1996).

3. One sex (usually males) has a greater variance in reproductive success with quality. Though the reproductive potential of both males and females may vary with quality, one sex varies more than the other, depending on the relative advantages of high quality males and females. Although reproductive success is often framed in terms of number of offspring, Leimar (1996) showed that reproductive values are more relevant for sex ratio evolution. As described in Section 5.6.2, we express the notion of "variance in reproductive success" in terms of male and female reproductive value ratios.

In polygynous ungulates, for example, males have the greater reproductive variance. Dominant high quality males monopolize breeding opportunities and have many more offspring than low quality males, while high quality females have only moderately more offspring than low quality females (Trivers and Willard 1973). In other species, females have the greater reproductive variance. Female baboons and macaques, for example, are more strongly affected by maternal quality due to their inheritance of maternal rank. As a result, the sex ratios of high-ranking mothers are biased towards female offspring (Silk 1983). 


\subsubsection{Model}

Trivers and Willard based their analysis on a verbal argument that implicitly relies on the principle of equal investment. Here, we explore similar questions in a structured model that includes multiple stages, qualities, and pair formation.

The population in our model consists of male and female low quality juveniles $\left(m_{01}, f_{01}\right)$, high quality juveniles $\left(m_{02}, f_{02}\right)$, low quality adults $\left(m_{1}, f_{1}\right)$, and high quality adults $\left(m_{2}, f_{2}\right)$. Low and high quality adults interbreed to form four types of unions, as in (5.23).

The population vector (5.2) has 12 stages total:

$$
\mathbf{n}(t)=\left(\begin{array}{llllllllllll}
m_{01} & m_{02} & m_{1} & m_{2} & f_{01} & f_{02} & f_{1} & f_{2} & u_{11} & u_{21} & u_{12} & u_{22}
\end{array}\right)^{\top}
$$

Again, we will write a model of the form (5.4), and the next three sections give the matrices $\mathbf{B}$, $\mathbf{U}$, and $\mathbf{T}$ in turn.

\subsubsection{Births (B)}

Unlike Case 1, offspring do not have different mortality rates or impose parental mortality. However, the production of male and female offspring requires different amounts of resources, as in Shyu and Caswell (xxb , Case 1). Producing a male offspring costs $C_{m}$ units of resources per time, while a female offspring costs $C_{f}$ units of resources per time. Each union's total rate of resource investment in offspring production is determined by maternal quality, where:

$$
\begin{aligned}
& R_{1}=\text { rate of resource investment by low quality females } \\
& R_{2}=\text { rate of resource investment by high quality females }
\end{aligned}
$$

Because high quality females have more resources for producing offspring, $R_{2}>R_{1}$. The corresponding low and high quality female reproductive rates, $k_{1}\left[R_{1}\right]$ and $k_{2}\left[R_{2}\right]$, are given by (5.26).

Let $q_{i j}$ be the probability that a female of quality $j$ produces quality $i$ offspring. We assume inheritance of quality, in that mothers are equally or more likely to produce offspring of the same quality. Thus, the $q_{i j}$ must satisfy the following conditions:

$$
\begin{aligned}
& q_{11}+q_{21}=1 \\
& q_{12}+q_{22}=1 \\
& q_{22}>q_{12} \rightarrow q_{22} \geq 0.5 \\
& q_{11}>q_{21} \rightarrow q_{11} \geq 0.5
\end{aligned}
$$


The complete birth matrix $\mathbf{B}$ is:

$$
\mathbf{B}=\left(\begin{array}{cccccccccccc}
0 & 0 & 0 & 0 & 0 & 0 & 0 & 0 & s_{1} k_{1}\left[R_{1}\right] q_{11} & s_{1} k_{1}\left[R_{1}\right] q_{11} & s_{2} k_{2}\left[R_{2}\right] q_{12} & s_{2} k_{2}\left[R_{2}\right] q_{12} \\
0 & 0 & 0 & 0 & 0 & 0 & 0 & 0 & s_{1} k_{1}\left[R_{1}\right] q_{21} & s_{1} k_{1}\left[R_{1}\right] q_{21} & s_{2} k_{2}\left[R_{2}\right] q_{22} & s_{2} k_{2}\left[R_{2}\right] q_{22} \\
0 & 0 & 0 & 0 & 0 & 0 & 0 & 0 & 0 & 0 & 0 & 0 \\
0 & 0 & 0 & 0 & 0 & 0 & 0 & 0 & 0 & 0 & 0 & 0 \\
0 & 0 & 0 & 0 & 0 & 0 & 0 & 0 & \left(1-s_{1}\right) k_{1}\left[R_{1}\right] q_{11} & \left(1-s_{1}\right) k_{1}\left[R_{1}\right] q_{11} & \left(1-s_{2}\right) k_{2}\left[R_{2}\right] q_{12} & \left(1-s_{2}\right) k_{2}\left[R_{2}\right] q_{12} \\
0 & 0 & 0 & 0 & 0 & 0 & 0 & 0 & \left(1-s_{1}\right) k_{1}\left[R_{1}\right] q_{21} & \left(1-s_{1}\right) k_{1}\left[R_{1}\right] q_{21} & \left(1-s_{2}\right) k_{2}\left[R_{2}\right] q_{22} & \left(1-s_{2}\right) k_{2}\left[R_{2}\right] q_{22} \\
0 & 0 & 0 & 0 & 0 & 0 & 0 & 0 & 0 & 0 & 0 & 0 \\
0 & 0 & 0 & 0 & 0 & 0 & 0 & 0 & 0 & 0 & 0 & 0 \\
0 & 0 & 0 & 0 & 0 & 0 & 0 & 0 & 0 & 0 & 0 & 0 \\
0 & 0 & 0 & 0 & 0 & 0 & 0 & 0 & 0 & 0 & 0 & 0 \\
0 & 0 & 0 & 0 & 0 & 0 & 0 & 0 & 0 & 0 & 0 & 0 \\
0 & 0 & 0 & 0 & 0 & 0 & 0 & 0 & 0 & 0 & 0 & 0
\end{array}\right)
$$

\subsubsection{Union formation (U)}

Each union type $u_{i j}$ is formed at a mating rate $U_{i j}$ determined by the mating preference functions described in Section 5.3.2. Assume that males are not picky in their choice of females, so that the male preference distribution is given by the random mating preference (5.10). However, as per our first assumption, females may prefer to mate with high quality males. Thus, the female preference distribution will be given by the biased mating preference (5.11).

Low and high quality males have competitiveness factors $c_{1}$ and $c_{2}$ respectively. Since high quality males are more likely to obtain mates than their low quality counterparts, $c_{2}>c_{1}$. Because $c_{1}+c_{2}=1$ in accordance with (5.8):

$$
c_{2}>c_{1} \rightarrow c_{1}<0.5
$$

Using the harmonic mean mating function (5.13), the per capita mating functions (5.14) become:

$$
\begin{aligned}
U_{m 11} & =\frac{2 c_{1} f_{1}}{c_{1}\left(f+m_{1}\right)+c_{2} m_{2}} & U_{f 11} & =\frac{2 c_{1} m_{1}}{c_{1}\left(f+m_{1}\right)+c_{2} m_{2}} \\
U_{m 21} & =\frac{2 c_{2} f_{1}}{c_{1} m_{1}+c_{2}\left(f+m_{2}\right)} & U_{f 21} & =\frac{2 c_{2} m_{2}}{c_{1} m_{1}+c_{2}\left(f+m_{2}\right)} \\
U_{m 12} & =\frac{2 c_{1} f_{2}}{c_{1}\left(f+m_{1}\right)+c_{2} m_{2}} & U_{f 12} & =\frac{2 c_{1} m_{1}}{c_{1}\left(f+m_{1}\right)+c_{2} m_{2}} \\
U_{m 22} & =\frac{2 c_{2} f_{2}}{c_{1} m_{1}+c_{2}\left(f+m_{2}\right)} & U_{f 22} & =\frac{2 c_{2} m_{2}}{c_{1} m_{1}+c_{2}\left(f+m_{2}\right)}
\end{aligned}
$$

where $m=m_{1}+m_{2}$ and $f=f_{1}+f_{2}$. 
The union matrix $\mathbf{U}$ is:

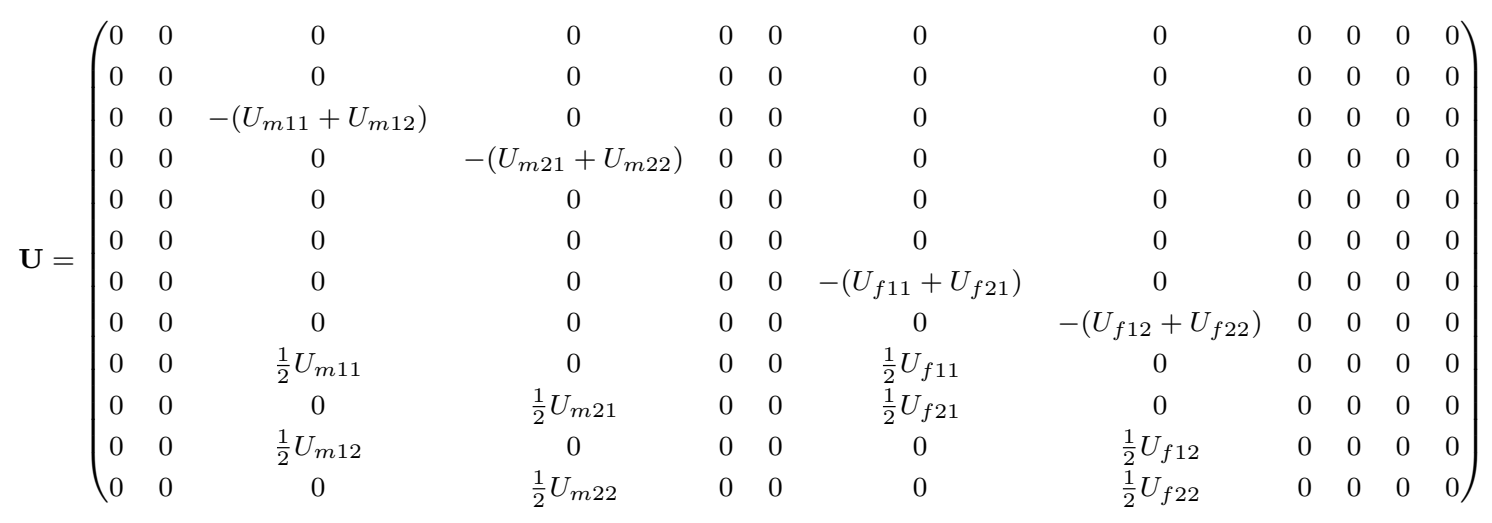

\subsubsection{Transitions $(\mathrm{T})$}

Mortality rates are the same for individuals of the same sex and quality, regardless of whether they are in unions. Again, unions dissolve due to divorce rates $d_{i j}$ or partner deaths (with mortality rates $\mu_{m 1}, \mu_{f 1}, \mu_{m 2}$, and $\left.\mu_{f 2}\right)$.

Low quality juveniles mature into low quality adults at a rate $\alpha_{m 1}$ for males and $\alpha_{f 1}$ for females. High quality juveniles mature into high quality adults at a rate $\alpha_{m 2}$ for males and $\alpha_{f 2}$ for females. Individuals cannot transition between different qualities.

The transition matrix is $\mathbf{T}=\left[\mathbf{T}_{1} \mid \mathbf{T}_{2}\right]$ where:

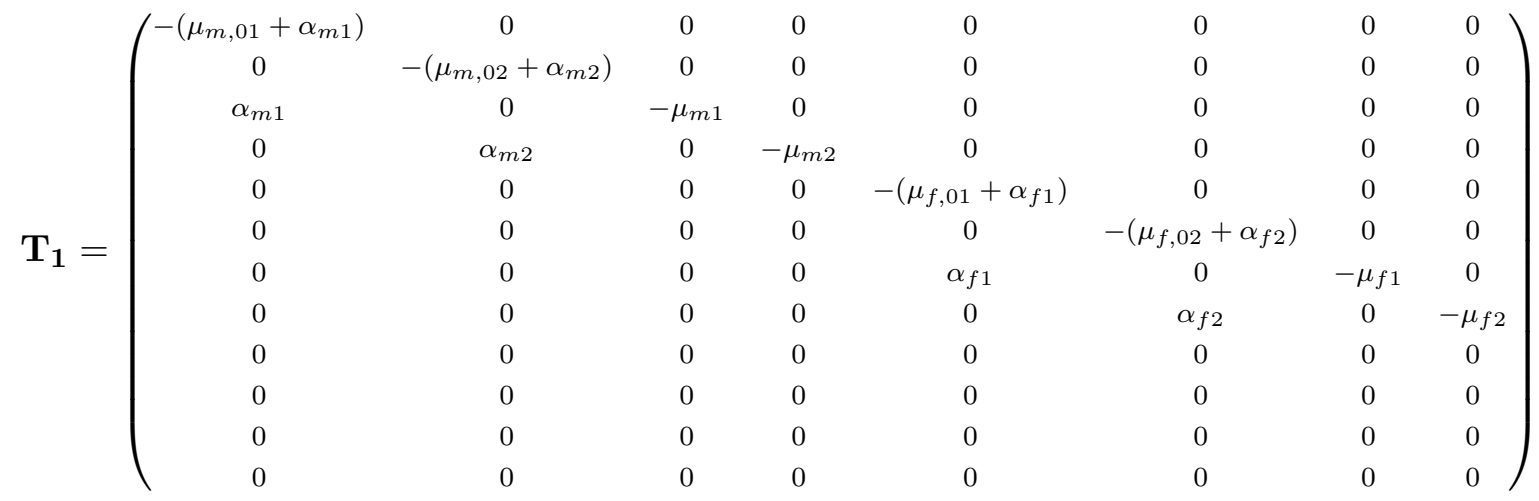

$$
\begin{aligned}
& \mathbf{T}_{\mathbf{2}}=\left(\begin{array}{cccc}
0 & 0 & 0 & 0 \\
0 & 0 & 0 & 0 \\
\left(\mu_{f 1}+d_{11}\right) & 0 & \left(\mu_{f 2}+d_{12}\right) & 0 \\
0 & \left(\mu_{f 1}+d_{21}\right) & 0 & 0 \\
0 & 0 & 0 & 0 \\
0 & 0 & 0 & 0 \\
\left(\mu_{m 1}+d_{11}\right) & \left(\mu_{m 2}+d_{21}\right) & \left(\mu_{m 1}+d_{12}\right) & \left(\mu_{m 2}+d_{22}\right) \\
0 & 0 & 0 & 0 \\
-\left(\mu_{m 1}+\mu_{f 1}+d_{11}\right) & 0 & 0 & 0 \\
0 & -\left(\mu_{m 2}+\mu_{f 1}+d_{21}\right) & -\left(\mu_{m 1}+\mu_{f 2}+d_{12}\right) & 0 \\
0 & 0 & 0 & -\left(\mu_{m 2}+\mu_{f 2}+d_{22}\right)
\end{array}\right)
\end{aligned}
$$




\subsubsection{Calculating variance in reproductive success}

Recall that the Trivers-Willard hypothesis requires individuals of different qualities (here, high and low quality), a correlation between parent and offspring quality $(q \geq 0.5)$, and that one sex has a greater variance (larger differential) in reproductive success with quality.

The hypothesis predicts that high quality females preferentially invest in the sex with the greater variance in reproductive success (Trivers and Willard 1973). Testing this hypothesis requires two quantities: a measure of reproductive success for each sex, and a measure of the "variance" in reproductive success (i.e., how much reproductive success varies between high and low quality individuals of a given sex).

\subsubsection{Reproductive success}

Though reproductive success is sometimes measured as number of offspring (Clutton-Brock et al. 1984, 1986), Leimar (1996) showed that reproductive value (the present value of all future offspring) was a more relevant index of reproductive success. This is especially true when the probability of maternal quality transmission is high; if females are more likely than males to pass their quality to offspring, a high quality female may still have high reproductive success, in that her reproductive value is large even if her number of offspring is not (West 2009).

Here, we use the demographic definition of reproductive value, which depends on the matrix model. Specifically, the dominant left eigenvector $\mathbf{v}$ of the projection matrix $\mathbf{A}(\hat{\mathbf{p}})$ is a vector of stage-specific reproductive values (shown in age-structured models by Goodman 1968; extended to stage-structured models by Caswell and Werner 1978 and others). Entry $i$ of $\mathbf{v}$ corresponds to the reproductive value $v_{i}$ of stage $i$.

Although different stages of a given sex will have different reproductive values, the juvenile (newborn) reproductive values should be the most relevant indices of reproductive success for each sex (Appendix 5.A).

\subsubsection{Variance in reproductive success}

The "variance" in reproductive success can be written as the juvenile male and female reproductive value ratios (RVRs) at equilibrium (Leimar 1996). Note that reproductive values are defined only up to a multiplicative constant, so taking the ratios of reproductive values removes this constant factor.

Define the male reproductive value ratio $M R V R$ as:

$$
M R V R=\frac{v_{m, 02}}{v_{m, 01}}
$$

where $v_{m, 02}$ and $v_{m, 01}$ are the reproductive values of high and low quality juvenile males respectively. 
Similarly, the female reproductive value ratio $F R V R$ is:

$$
F R V R=\frac{v_{f, 02}}{v_{f, 01}}
$$

where $v_{m, 02}$ and $v_{m, 01}$ are the reproductive values of high and low quality juvenile females respectively.

We will use $M R V R$ and $F R V R$ to represent the variance in reproductive success (between high and low quality individuals) for males and females respectively.

\subsubsection{Predictions of the Trivers-Willard hypothesis}

When $M R V R>F R V R$ (males have greater variance in reproductive success than females), high quality mothers should be more likely than low quality mothers to produce sons. As a result, we would expect that $s_{2}>s_{1}$. Given the biological constraints (5.19), one of the following two cases should thus occur (Leimar 1996).

$$
\begin{aligned}
& s_{1}=0,0<s_{2}<1 \\
& 0<s_{1}<1, s_{2}=1
\end{aligned}
$$

When $F R V R>M R V R$ (females have greater variance in reproductive success than males), high quality mothers should be more likely than low quality mothers to produce daughters. As a result, we would expect that $s_{2}<s_{1}$, and that one of the following two cases should occur (Leimar 1996).

$$
\begin{aligned}
& s_{1}=1,0<s_{2}<1 \\
& 0<s_{1}<1, s_{2}=0
\end{aligned}
$$

\subsubsection{Results}

As described at the beginning of Section 5.6, we consider two advantages that high quality individuals may have over low quality individuals. High quality males may be more attractive and competitive mates than low quality males $\left(c_{2}>c_{1}\right)$, which affects the male variance in reproductive success. Alternatively, high quality females may be more productive and have a greater resource investment rate than low quality females $\left(R_{2}>R_{1}\right)$, which affects the female variance in reproductive success. We will determine how $\mathbf{s}^{*}$ evolves in both cases.

\subsubsection{Male variance in reproductive success}

Male variance in reproductive success depends on the difference between the low quality male competitiveness factor $c_{1}$ from (5.40), and the high quality male competitiveness factor $c_{2}=1-c_{1}$. Because high quality males obtain more matings, $c_{2}>c_{1}$. As $c_{1}$ increases ( $c_{2}$ decreases), the 
proportion of females mating with a low quality male (not mating with a high quality male) also increases.

Figure 5-1a shows how $M R V R, F R V R$, and $\mathbf{s}^{*}$ vary with $c_{1}$. On the left side of the $\mathrm{x}$-axis, males have high variance in competitive ability $\left(c_{1}=0.01, c_{2}=0.99\right)$; on the right side of the $\mathrm{x}$-axis, males have no variance in competitive ability $\left(c_{1}=c_{2}=0.5\right)$. As a result, the variance in male reproductive success, as given by $M R V R$, is highest on the left and converges to 1 on the right.

We have assumed that high and low quality females are equally productive $\left(R_{2}=R_{1}\right)$, so that there is almost no variance in female reproductive success $(F R V R \approx 1)$. As a result, $M R V R \geq$ $F R V R$ at all $\mathbf{s}^{*}$. However, note that, at low $c_{1}, F R V R$ is slightly greater than 1 , indicating that high quality females are somewhat more successful than low quality females (because they are more likely to produce high quality offspring).

As predicted by the Trivers-Willard hypothesis, low quality mothers produce relatively more of the sex with lower variance in reproductive success, while high quality mothers produce more of the higher variance sex. In this case, the sex ratio of high quality mothers favors exclusively males $\left(s_{2}^{*}=1\right)$, while the sex ratio of low quality mothers is female-biased $\left(s_{1}^{*}<0.5\right)$. When $c_{1}=c_{2}=0.5$, $M R V R=F R V R=1$ and equal sex ratios for both $s_{1}^{*}$ and $s_{2}^{*}$ can occur.

\subsubsection{Female variance in reproductive success}

Female variance in reproductive success is affected by the difference between the low and high quality female resource investment rates, $R_{1}$ and $R_{2}$ from (5.37). Again, high quality females should have more resources for offspring production $\left(R_{2}>R_{1}\right)$.

Figure 5-1b shows how $M R V R, F R V R$, and $\mathbf{s}^{*}$ vary with $R_{1}$. We set $R_{2}=30-R_{1}$, so that the left side of the $\mathrm{x}$-axis corresponds to a high variance in female resource investment $\left(R_{1}=0.25, R_{2}=\right.$ $29.75)$, and the right side corresponds to no variance in female resource investment $\left(R_{1}=R_{2}=15\right)$. Thus, $F R V R$, is highest on the left and converges to 1 on the right. We assume that high and low quality males do not differ $\left(c_{2}=c_{1}\right)$, so that $M R V R=1$ always. In this case, $F R V R \geq M R V R$ at all $\mathbf{s}^{*}$; that is, females always have the greater variance in reproductive success.

Again, consistent with the Trivers-Willard effect, high quality mothers favor the higher variance sex (females). While high quality mothers produce relatively more high variance female offspring $\left(s_{2}^{*}<0.5\right)$, low quality mothers produce all low variance male offspring $\left(s_{1}^{*}=1\right)$. Although low quality females are relatively unproductive, all males are equally likely to mate with high quality females and produce high quality grandchildren. As a result, it appears that low quality mothers evolve to maximize their sons.

\subsubsection{The effect of quality inheritance}

Lastly, we consider how $\mathbf{s}^{*}$ is affected by the quality inheritance probability $q_{i j}$ in (5.38). We assume that quality depends only on mothers, which produce offspring of the same quality with a probability 
a) effect of male competitive ability
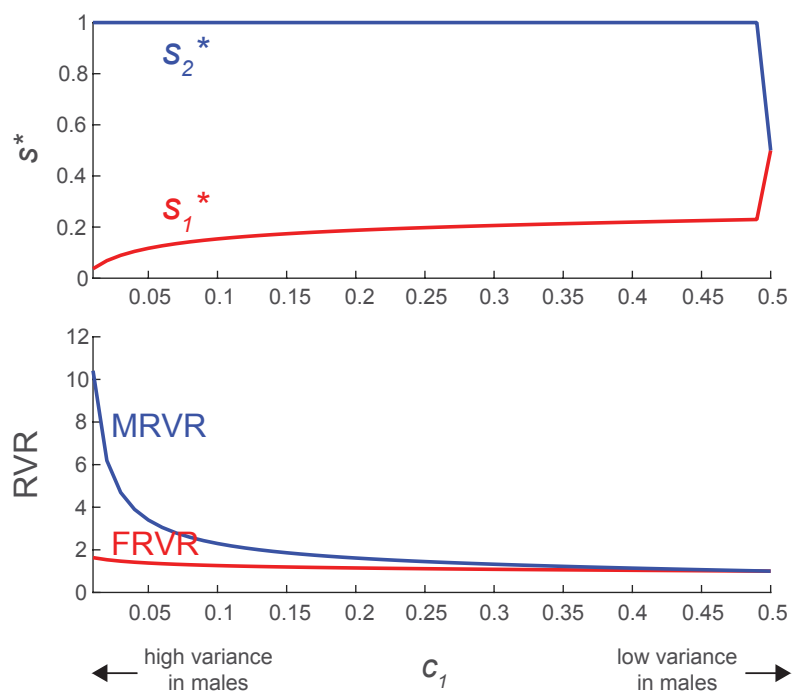

b) effect of female resource investment
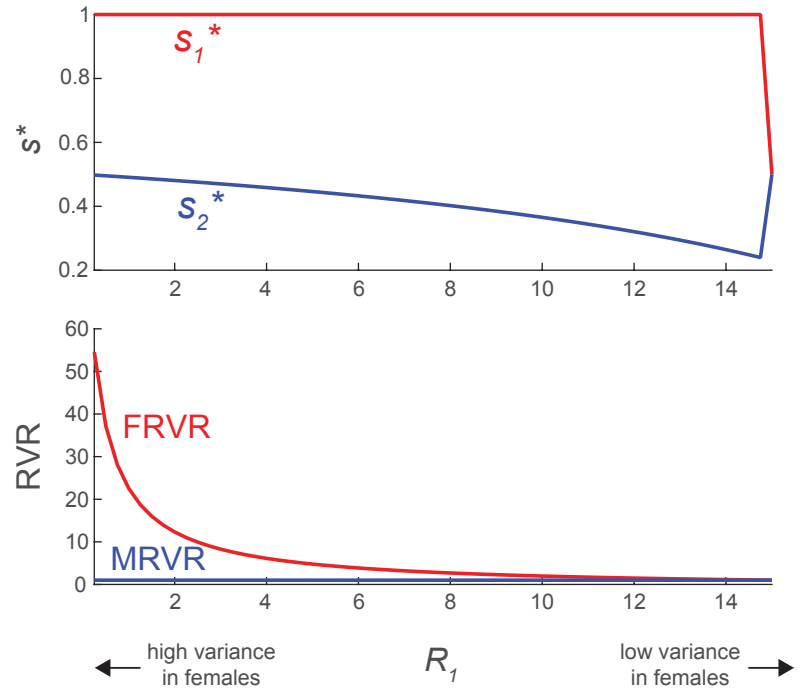

Figure 5-1: Singular strategies $\mathbf{s}^{*}$ ( $s_{1}^{*}$ for low quality females, $s_{2}^{*}$ for high quality females) and reproductive value ratios $M R V R, F R V R$ as functions of a) the low quality male competitiveness factor $c_{1}$, where the high quality male competitiveness factor is $c_{2}=1-c_{1}$ (with $R_{1}=R_{2}=15$ ), and $\mathbf{b}$ ) the low quality female investment rate $R_{1}$, where the high quality female resource investment rate $R 2=30-R_{1}$ (with $c_{1}=c_{2}=0.5$ ). Other model parameters are as given in Table 5.1.

$q=q_{j j}>0.5$. An increase in $q$ increases the value of high quality mothers, because they are more likely to generate high quality offspring. When $q$ is high, high quality females can become very valuable, leading high quality mothers to prefer daughters over sons (Leimar 1996).

We also include both the male advantage and female advantages of high quality individuals; that is high quality males are more competitive $\left(c_{2}>c_{1}\right)$ and high quality females are more fertile $\left(R_{2}>R_{1}\right)$ Which sex has the larger reproductive variance now varies with $q$.

As shown in Figure 5-2, at low $q$, the variance in reproductive success of males exceeds that of females $(M R V R>F R V R)$. High quality mothers thus have male-biased sex ratios; low quality mothers, in contrast, produce exclusively daughters. At intermediate $q$, there is an interval where $M R V R=F R V R$ corresponding to interior SSs of $\mathbf{s}^{*}$. At higher $q$, females become the sex with higher variance in reproductive success $(F R V R>M R V R)$. High quality mothers ultimately converge to the equal sex ratio $s_{2}=0.5$, while low quality mothers produce only sons.

These results are consistent with the predictions of the Trivers-Willard hypothesis, in that high quality mothers always produce more the sex with greater variance in reproductive value than lower quality mothers do. At intermediate values of $q$, however, there is a region where $M R V R=F R V R$; this corresponds to interior (Type 1) SSs where both $s_{1}$ and $s_{2}$ are between 0 and 1 (see Section 5.6.3.4). Because males and females have the same reproductive value ratios at interior SSs, it is not obvious from the Trivers-Willard hypothesis which sex the high quality parents will favor. 

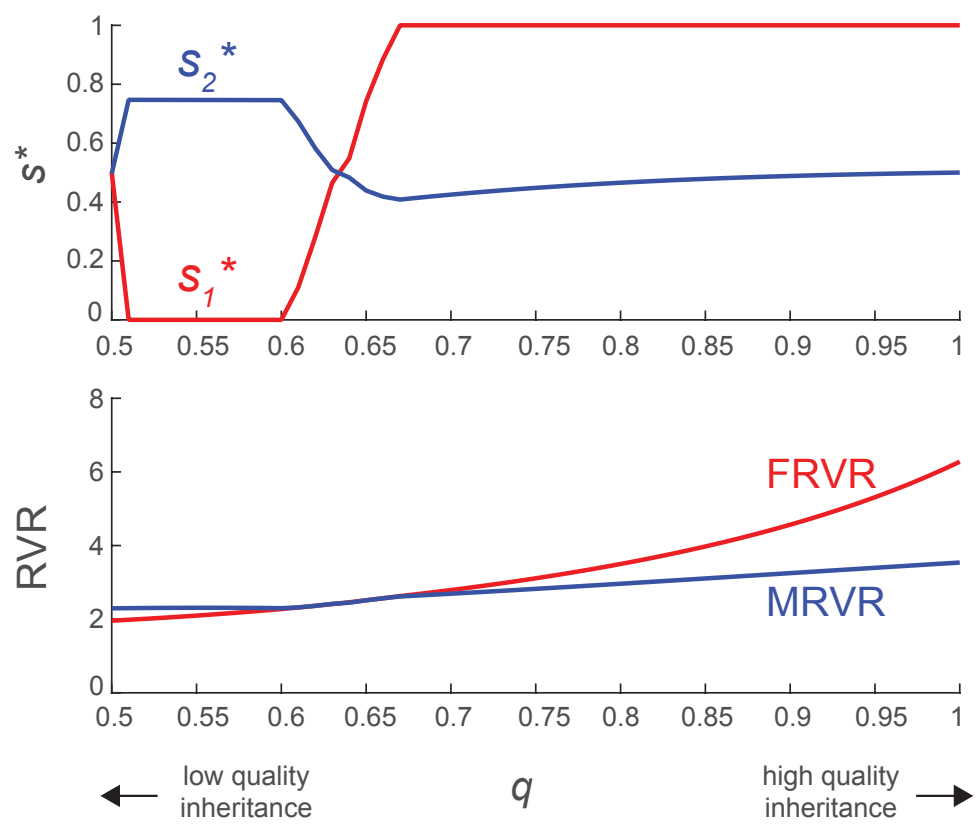

Figure 5-2: Singular strategies $\mathbf{s}^{*}$ ( $s_{1}^{*}$ for low quality females, $s_{2}^{*}$ for high quality females) and reproductive value ratios $M R V R, F R V R$ as functions of the same quality inheritance probability $q=q_{j j}$. Both the male advantage $\left(c_{2}>c_{1}\right)$ and female advantage $\left(R_{2}>R_{1}\right)$ are present, with $c_{1}=0.1, c_{2}=0.9$ and $R_{1}=10, R_{2}=20$. Other model parameters are as given in Table 5.1.

\subsubsection{Reproductive value ratios and the nature of $\mathrm{s}^{*}$}

In this section, we determine the reproductive value ratios at equilibrium for each type of SS $\mathbf{s}^{*}$, and their implications for the Trivers-Willard hypothesis. Recall that there are five types of $\mathbf{s}^{*}$ in this model (an interior point and four boundary equilibria), each of which implies different conditions for the selection gradients (derivatives of invasion fitness) at equilibrium (summarized in Figure 5-2). These selection gradients, in turn, depend on the reproductive values at equilibrium (the left eigenvector $\mathbf{v}^{\prime}$ ) through (5.16). This permits us to invert the calculations presented thus far, which focus on finding $\mathbf{s}^{*}$ in various scenarios. We now determine the relationship between male and female reproductive value ratios, given each possible type of $\mathbf{s}^{*}$.

In Appendix 5.A, we find the relationship between the male and female reproductive value ratios $M R V R$ (5.44) and $F R V R$ (5.45) at each type of $\mathbf{s}^{*}$. These relationships are summarized in Table 5.1. Because the RVRs are measures of the variance in reproductive success for each sex (Section 5.6.2.2), each type of SS therefore also implies a certain relationship between male and female reproductive success. A Type $2 \mathrm{SS}$, for example, requires that $M R V R<F V R V$ - that is, the variance in reproductive success of females must exceed that of males.

Each of the five types of $\mathbf{s}^{*}$ also has a certain biological interpretation (Table 5.2). At Type 3 and $4 \mathrm{SSs}$, high quality mothers will produce relatively more sons than low quality mothers do; at Type 2 and $5 \mathrm{SSs}$, high quality mothers will produce more daughters. This allows us to link 


\begin{tabular}{llllll}
\hline Type of SS & $\begin{array}{l}\text { Low quality } \\
\text { sex ratio }\left(s_{1}\right)\end{array}$ & $\begin{array}{l}\text { High quality } \\
\text { sex ratio }\left(s_{2}\right)\end{array}$ & $\begin{array}{l}\text { Reproduction } \\
\text { value ratios }\end{array}$ & $\begin{array}{l}\text { Offspring cost } \\
\text { ratios }\end{array}$ & Examples \\
\hline 1 (interior) & $0<s_{1}<1$ & $0<s_{2}<1$ & $M R V R=F R V R$ & $\frac{C_{m}}{C_{f}}=\frac{v m_{01}}{v_{f, 01}}=\frac{v m_{02}}{v_{f, 02}}$ & Figure 5-2 (mid $q)$ \\
\hline 2 (boundary) & $0<s_{1}<1$ & 0 (all females) & $M R V R<F R V R$ & $\frac{C_{m}}{C_{f}}<\frac{v m_{01}}{v_{f, 01}},>\frac{v m_{02}}{v_{f, 02}}$ & Not observed \\
\hline 3 (boundary) & $0<s_{1}<1$ & 1 (all males) & $M R V R>F R V R$ & $\frac{C_{m}}{C_{f}}>\frac{v m_{01}}{v_{f, 01}},<\frac{v m_{02}}{v_{f, 02}}$ & Figure 5-1a (all $\left.c_{1}\right)$ \\
\hline 4 (boundary) & 0 (all females) & $0<s_{2}<1$ & $M R V R>F R V R$ & $\frac{C_{m}}{C_{f}}>\frac{v m_{01}}{v_{f, 01}},<\frac{v m_{02}}{v_{f, 02}}$ & Figure 5-2 (low $q)$ \\
\hline 5 (boundary) & 1 (all males) & $0<s_{2}<1$ & $M R V R<F R V R$ & $\frac{C_{m}}{C_{f}}<\frac{v m_{01}}{v_{f, 01}},>\frac{v m_{02}}{v_{f, 02}}$ & $\begin{array}{l}\left.\text { Figure 5-1b (all } R_{1}\right) \\
\text { Figure 5-2 (high } q)\end{array}$ \\
\hline
\end{tabular}

Table 5.1: Relationships between the male and female reproductive value ratios MRVR and FRVR at each of the five possible singular strategies $\mathbf{s}^{*}$ (in Figure 5-2.

\begin{tabular}{llcc}
\hline $\begin{array}{l}\text { High quality } \\
\text { mothers } \\
\text { have more: }\end{array}$ & Sex ratios & Type of SS & Greater RVR \\
\hline sons & $s_{1}=0,0<s_{2}<1$ & 4 & MRVR \\
& $0<s_{1}<1, s_{2}=1$ & 3 & \\
daughters & $s_{1}=1,0<s_{2}<1$ & 5 & $F R V R$ \\
& $0<s_{1}<1, s_{2}=0$ & 2 & $M R V R=F R V R$ \\
either & $0<s_{1}<1,0<s_{2}<1$ & 1 & $M R R$ \\
\hline
\end{tabular}

Table 5.2: How the sex preferred by high quality mothers corresponds to different sex ratios, types of SS (Figure 5-2), and reproductive value ratio relationships. The first two cases correspond to the conditions (5.46) and the second two cases correspond to conditions (5.47).

variance in reproductive success, as given by $M R V R$ and $F R V R$, to the sex favored by high quality mothers, as invoked by the Trivers-Willard hypothesis.

As shown in Appendix 5.A, we find that high quality mothers consistently favor sons when $M R V R>F R V R$ (Type 3 or $4 \mathrm{SS}$ ), and daughters when $F R V R>M R V R$ (Type 2 or 5 SS). These results confirm a Trivers-Willard effect in our model, and are similar to those of Leimar's simpler model (1996), which does not consider juvenile or union stages. We also find that when high quality mothers produce exclusively one sex (Types 2 and $3 \mathrm{SSs}$ ), they always favor the sex with the greater reproductive value ratio. However, when low quality mothers produce exclusively one sex (Type 4 and $5 \mathrm{SSs}$ ), they always favor the sex with the lower reproductive value ratio.

Our results demonstrate the presence of a "specialization principle" - unless $M R V R=F R V R$ at equilibrium, one maternal quality will produce all sons or all daughters (i.e., have a Type $2-5$ boundary SS). The RVRs are only equal at Type 1 (interior) SSs, where high quality mothers may favor either sex. Interior SSs are unique in that they do not experience selective pressure in any direction, because the selection gradients are zero for both sex ratios. This suggests that selective pressure only ceases completely when both male and female reproductive value ratios are equal $(M R V R=F R V R)$. Interestingly, we do not find the infinite equilibria predicted by Leimar (1996) when $M R V R=F R V R$. 
Interior $\mathbf{s}^{*}$ are also the only type of SS where the ratio of juvenile male to juvenile female reproductive values equals the ratio of the sex-specific resource costs. Specifically, by (5.53):

$$
\frac{C_{m}}{C_{f}}=\frac{v_{m, 01}}{v_{f, 01}}=\frac{v_{m, 02}}{v_{f, 02}}
$$

This result holds true for both low quality juveniles $\left(v_{m, 01} / v_{f, 01}\right)$ and high quality juveniles $\left(v_{m, 02} / v_{f, 02}\right)$. A similar result for the SS of a single sex ratio was found in Shyu and Caswell (xxb, Case 1). As in the single sex ratio case, this suggests that the sex ratios evolve towards an "equal investment principle," where the ratio of male to female reproductive values equals to the ratio of the sex-specific resource costs. If, however, such a point does not exist within the biologically-constrained region, $\mathbf{s}^{*}$ becomes a boundary SS and equal investment no longer holds (Table 5.1, "Offspring costs" column).

\subsection{Discussion}

When a trait like the primary sex ratio varies with an individual's condition, the evolution of that trait may be difficult to anticipate. Because multiple conditions create population structure, and the reproductive advantages of both sexes depends on demographic factors like survival, fecundity, and lifespan (Leimar 1996, Schwanz 2006), an explicitly demographic model is valuable for understanding facultative sex ratio evolution.

We have presented several two-sex demographic models that include two maternal conditions with different sex ratios. The overall sex ratio strategy $\mathbf{s}$ is thus a vector trait with two simultaneously evolving components. Using multidimensional adaptive dynamics, we analyzed both the transient and long-term evolution of $\mathbf{s}$ in cases where individuals differed in age or quality.

In our models, $\mathbf{s}$ displays a wide range of evolutionary behavior. The sex ratio strategy may evolve to an interior SS where both $s_{1}$ and $s_{2}$ are between 0 and 1, or a boundary SS where either $s_{1}$ or $s_{2}$ is 0 or 1 (i.e., mothers of a particular condition produce exclusively one sex). Previous models of facultative sex ratios have similarly found cases where at least one maternal condition only produces offspring of a single sex (e.g., Leimar 1996, Schwanz 2006).

We have also found cases where $\mathbf{s}$ converges to a line of selectively neutral strategies. This potential for infinitely many sex ratio combinations may lend insight into why empirical studies have observed many different, and occasionally contradictory, relationships between sex ratios and maternal conditions (e.g., Jacobsen et al. 1999 in humans, Sheldon and West 2004 in ungulates). Ultimately, our model lends insight into the demographic factors that cause different types of evolutionary singular strategies, and, in the case of multiple qualities, their relationships with the reproductive values that underlie the Trivers-Willard hypothesis.

Although we considered only two conditions at a time (i.e., young and old, high and low quality), in reality, individuals will vary across a spectrum of conditions. Our matrix model could 
be expanded to accommodate more stages for additional conditions, though continuously-varying traits and conditions may require an alternative approach. Our model also assumes that mating preferences are proportional to the relative abundances (or weighted abundances) of adult stages, through functions like (5.10) and (5.11). Ranking systems where mating preferences depend on the overall composition of the population (e.g. females prefer the largest males currently available) are not explicitly covered by our formulation.

Several aspects of our model could be modified to study other scenarios. We assumed, for example, that the effects of mutations on the sex ratios of younger and older (or low and high quality) individuals were uncorrelated. However, a mutation in one gene may affect multiple traits through pleiotropic effects. Antagonistic pleiotropy, whereby selection promotes genes that are beneficial earlier in life, but detrimental later in life, may be an important factor in the development of senescence (Williams 1957). Charnov (1982) hypothesized that this may influence how sex ratios shift with maternal age - that is, factors reducing mortality from early reproduction might increase mortality due to later reproduction. While we found changes in age-specific sex ratios, even without accounting for these kinds of pleiotropic effects, one could explicitly incorporate mutational correlations by modifying the mutational variance matrix $\mathbf{V}(\mathbf{s})(5.20)$.

Although we have considered only the effects of maternal condition, paternal condition may also influence the primary sex ratio. Paternal attractiveness is of particular interest, in that females mated to attractive males may produce more sons to inherit their father's attractiveness. Resulting sex ratios depend on the nature of the female mating preference and costs and benefits of attractive male traits (Pen and Weissing 2000, Fawcett 2007, West 2009). Paternal age may also affect offspring sex ratios. Several large-scale studies on human populations, for instance, have found more significant correlations between sex ratios and paternal ages than sex ratios and maternal ages (reviewed in Jacobsen et al. 1999). We previously assumed that any union $u_{i j}$ with a Condition $j$ female has sex ratio $s_{j}$; that is, the primary sex ratio depends only on the maternal condition. However, our model could easily be modified to have sex ratios depend on the paternal condition as well.

Lastly, we do not consider any costs or mechanisms for switching between the facultative sex ratios $s_{1}$ and $s_{2}$. Costly sex ratio manipulation, e.g., via selective abortion, may significantly affect sex ratio evolution (Pen and Weissing 2002), and cases where one parental condition uses a very different sex ratio from the other may be less feasible if there are high costs for switching sex ratios. There may also be biological limits to how much the sex ratio can be adjusted. Though actual mechanisms for sex-ratio adjustment are still largely unknown, glucose levels in utero may be an important factor (Cameron 2004). 


\subsection{Acknowledgments}

This work was supported by a National Science Foundation Graduate Research Fellowship under Grant 1122374, NSF Grants DEB1145017 and DEB1257545 (to H. Caswell), Advanced Grant 322989 from the European Research Council (to H. Caswell), and the Woods Hole Oceanographic Institution Academic Programs Office.

\section{Bibliography}

[1] Almagor M, Schwed P, Yaffe H. 1998. Male to female ratio in newborns of grand grand multiparous women. Human Reproduction. 13: 2323-2324.

[2] Cameron EZ. 2004. Facultative adjustment of mammalian sex ratios in support of the Trivers-Willard hypothesis: evidence for a mechanism. Proceedings of the Royal Society B. 271: $1723-1728$.

[3] Cameron EZ, Dalerum F. 2009. A Trivers-Willard effects in contemporary humans: male-biased sex ratios among billionaires. PLoS One. 4(1): e4195. doi:10.1371/journal.pone.0004195

[4] Caswell H. 2010. Reproductive value, the stable stage distribution, and the sensitivity of the population growth rate to changes in vital rates. Demographic Research. 23: 531-548.

[5] Caswell H, Werner PA. 1978. Transient behavior and life history analysis of teasel (Dipsacus sylvestris Huds.). Ecology. 59: 53-66.

[6] Charlesworth B. 1977. Population genetics, demography, and the sex ratio. Measuring Selection in Natural Populations. Lecture Notes in Biomathematics. 19: 345-363.

[7] Charnov EL. 1982. The Theory of Sex Allocation. Monographs in Population Biology. Princeton: Princeton University Press.

[8] Charnov EL, Los-den Hartogh RL, Jones WT, van den Assem J. 1981. Sex ratio evolution in a variable environment. Nature. 289: 27-33.

[9] Clout, MM, Elliot GP, Robertson BC. 2002. Effect of supplementary feeding on the offspring sex ratio of kakapo: a dilemma for the conservation of a polygynous parrot. Biological Conservation. 107, 13-18.

[10] Clutton-Brock TH, Albon SD, Guiness FE. 1984. Maternal dominance, breeding success, and birth sex ratios in red deer. Nature. 308: 358-360.

[11] Clutton-Brock TH, Albon SD, Guiness FE. 1986. Great expectations: dominance, breeding success, and offspring sex ratios in red deer. Animal Behavior. 34: 460-471.

[12] Dieckmann U, Heino M, Parvinen K. 2006. The adaptive dynamics of function-valued traits. Journal of Theoretical Biology. 241: 370-389.

[13] Dieckmann U, Law R. 1996. The dynamical theory of coevolution: a derivation from stochastic ecological processes. Journal of Mathematical Biology. 34: 579-612. 
[14] Doebeli M. 2011. Adaptive Diversification. Princeton: Princeton University Press.

[15] Durinx M, Metz JAJ, Meszéna G. 2008. Adaptive dynamics for physiologically structured population models. Journal of Mathematical Biology. 56: 673-742.

[16] Fawcett TW, Kuijper B, Pen I, Weissing FJ. 2007. Should attractive males have more sons? Behavioral Ecology. 18: 71-80.

[17] Fisher RA. 1930. The Genetical Theory of Natural Selection. Oxford: Oxford University Press.

[18] Gaulin, SJC, Robbins CJ. 1991. Trivers-Willard effect in contemporary North American society. American Journal of Physical Anthropology. 85: 61-69.

[19] Geritz SAH, Kidsi É, Meszéna G, Metz JAJ. 1998. Evolutionarily singular strategies and the adaptive growth and branching of the evolutionary tree. Evolutionary Ecology. 12: 35-57.

[20] Goodman LA. 1968. An elementary approach to the population projection-matrix, to the population reproductive value, and to related topics in the mathematical theory of population growth. Demography. 5: 382-409.

[21] Hadeler KP. 1989. Pair formation in age-structured populations. Acta Applicandae Mathematicae. 14: 91-102.

[22] Hamilton WD. 1967. Extraordinary sex ratios. Science. 156: 477-488.

[23] Helle S, Lummaa V, Jokela J. 2002. Sons reduced maternal longevity in preindustrial humans. Science. 296: 1085.

[24] Hewison AJM, Gaillard J. 1999. Successful sons or advantaged daughters? The Trivers-Willard model and sex-biased maternal investment in ungulates. Trends in Ecology and Evolution. 14: 229-234.

[25] Jacobsen R, Møller H, Mouritsen A. 1999. Natural variation in the human sex ratio. Human Reproduction. 14: 3120-3125.

[26] James WH, Rostron J. 1985. Parental age, parity and sex ratio in births in England and Wales, 1968-77. Journal of Biosocial Science. 17: 47-56.

[27] Kilner R. 1998. Primary and secondary sex ratio manipulation by zebra finches. Animal Behavior. 56: 155-164.

[28] Lande R. 1979. Quantitative genetic analysis of multivariate evolution, applied to brain: body size allometry. Evolution. 33: 402-416.

[29] Leimar O. 1996. Life-history analysis of the Trivers and Willard sex-ratio problem. Behavioral Ecology. 7: 316-325.

[30] Lendete-Castillejos T, Gortázar C, Vincente J, Fierro Y, Garcia A, Gallego L. 2004. Age-related foetal sex ratio bias in Iberian red deer (Ceruvs elaphus hispanicus): are male calves too expensive for growing mothers? Behavioral Ecology and Sociobiology. 56: 1-8.

[31] Long TAF, Pischedda A. 2005. Do female Drosophila melanogaster adaptively bias offspring sex ratios in relation to the age of their mate? Proceedings of the Royal Society B. 272: 1781-1787. 
[32] Martcheva M, Milner F. 2001. The mathematics of sex and marriage, revisited. Mathematical Population Studies. 9: 123-141.

[33] Metz JAJ. 2006. Fitness. IIASA Interim Report IR-06-061. In Encyclopedia of Ecology (ed Jørgensen SE), pp.1599-1612, Elsevier (2008).

[34] Orzack SH, Stubblefield JW, Akmaev VR, Colls P, Munné S, Scholl T, Steinsaltz D, Zuckerman JE. 2015. The human sex ratio from conception to birth. PNAS. 112: E2102-E2111.

[35] Pen I, Weissing FJ. 2000. Sexual selection and the sex ratio: an ESS analysis. Selection. 1: 111-121.

[36] Pen I, Weissing FJ. 2002. Optimal sex allocation: steps towards a mechanistic theory. In Sex Ratios: Concepts and Research Methods (Ed. by ICW Hardy). Cambridge: Cambridge University Press. 26-45.

[37] Pen I, Weissing FJ, Daan S. 1999. Seasonal sex ratio trend in the European kestrel: an evolutionarily stable strategy analysis. American Naturalist. 153: 384-397.

[38] Pollard GN. 1969. Factors influencing the sex ratio at birth in Australia, 1902-65. Journal of Biosocial Science. 1: 125-144.

[39] Schwanz LE, Bragg JG, Charnov EL. 2006. Maternal condition and facultative sex ratios in populations with overlapping generations. American Naturalist. 168: 521-530.

[40] Sheldon, BC, West SA. 2004. Maternal dominance, maternal condition, and offspring sex ratio in ungulate mammals. American Naturalist. 163: 40-54.

[41] Shyu E, Caswell H. xxa. A stage-classified two-sex model incorporating multiple life cycle processes. In preparation.

[42] Shyu E, Caswell H. xxb. A demographic model for sex ratio evolution and the effects of sex-biased offspring costs. In preparation.

[43] Silk JB. 1983. Local resource competition and facultative adjustment of sex ratios in relation to competitive abilities. American Naturalist. 121: 56-66.

[44] Takahashi E. 1954. The effects of the age of the mother on the sex ratio at birth in Japan. Annals of the New York Academy of Sciences. 57: 531-550.

[45] Trivers RL. 1985. The primary sex ratio. In Social Evolution. Menlo Park: Benjamin-Cummings Publishing Company.

[46] Trivers RL, Willard DE. 1973. Natural selection of parental ability to vary the sex ratio of offspring. Science. 179: 90-92.

[47] Vatten LJ, Skjaerven R. 2004. Offspring sex and pregnancy outcome by length of gestation. Early Human Development. 76: 47-54.

[48] Wedekind C. 2002. Manipulation sex ratios for conservation: short-term risks and long-term benefits. Animal Conservation. 5: 13-20. 
[49] Werren JH, Charnov EL. 1978. Facultative sex ratios and population dynamics. Nature. 272: 349-350.

[50] West SA. 2009. Sex allocation. Princeton: Princeton University Press.

[51] Williams GC. 1957. Pleiotrophy, natural selection, and the evolution of senescence. Evolution. 11: 398-411. 


\section{Chapter 5 Appendix}

\section{A Reproductive value ratios at singular strategies}

In this section, we calculate the relation between $M R V R$ and $F R V R$ at each of the five types of singular strategies $\mathbf{s}^{*}$ in Figure 5-2. All SSs occur when one or both components of the selection gradient (5.17) is 0. As shown in (5.16), the selection gradient depends on the mutant reproductive value vector $\mathbf{v}^{\prime}$.

Given a $s \times 1$ population vector, the first term in (5.16) is the $1 \times s^{2}$ vector:

$$
\mathbf{w}^{\prime \top} \otimes \mathbf{v}^{\prime \top}=\left(\begin{array}{llll}
w_{1} v_{1} & w_{1} v_{2} & \ldots & w_{1} v_{s}
\end{array} \ldots \mid \begin{array}{lllll}
w_{s} v_{1} & w_{s} v_{2} & \ldots & w_{s} v_{s}
\end{array}\right)
$$

where $w_{i}$ is the $\mathrm{i}^{\text {th }}$ entry of $\mathbf{w}^{\prime}$ (stable stage frequency of stage $i$ ), and $v_{i}$ is the $\mathrm{i}^{\text {th }}$ entry of $\mathbf{v}^{\prime}$ (reproductive value of stage $i$ ).

The second term in (5.16) is the $s^{2} \times 2$ vector:

$$
\frac{d \operatorname{vec} \mathbf{A}^{\prime}}{d \mathbf{s}^{\prime}}=\frac{1}{3}\left(\frac{d \operatorname{vec} \mathbf{T}^{\prime}}{d \mathbf{s}^{\prime}}+\frac{d \operatorname{vec} \mathbf{B}^{\prime}}{d \mathbf{s}^{\prime}}+\frac{d \operatorname{vec} \mathbf{U}^{\prime}}{d \mathbf{s}^{\prime}}\right)
$$

We will use (5.42), (5.39), and (5.43)for the rate matrices $\mathbf{U}, \mathbf{B}$, and $\mathbf{T}$ respectively.

After substituting (5.49) and (5.50) into (5.16) and simplifying the results, we obtain the following expressions for the components of the selection gradient:

$$
\begin{aligned}
\frac{d \lambda^{\prime \top}}{d \mathbf{s}^{\prime}} & =\left(\begin{array}{l}
\left.\frac{\partial \lambda^{\prime}}{\partial s_{1}^{\prime}}\right|_{\mathbf{s}^{\prime}=\mathbf{s}} \\
\left.\frac{\partial \lambda^{\prime}}{\partial s_{2}^{\prime}}\right|_{\mathbf{s}^{\prime}=\mathbf{s}}
\end{array}\right) \\
& =\left(\begin{array}{cc}
C_{1} & 0 \\
0 & C_{2}
\end{array}\right)\left(\begin{array}{l}
-C_{m}\left[q v_{f, 01}+(1-q) v_{f, 02}\right]+C_{f}\left[q v_{m, 01}+(1-q) v_{m, 02}\right] \\
-C_{m}\left[(1-q) v_{f, 01}+q v_{f, 02}\right]+C_{f}\left[(1-q) v_{m, 01}+q v_{m, 02}\right]
\end{array}\right)
\end{aligned}
$$

where $C_{1}$ and $C_{2}$ are positive quantities that do not affect the signs or zeroes of the selection gradients.

At each type of SS, one or both components of the selection gradient (5.51) will be 0 . We will examine each of the five type of SS from Figure 5-2 to determine their corresponding reproductive value ratios. 


\section{A.1 Interior SS (Type 1)}

For an interior SS, both components of the selection gradient (5.51) are simultaneously 0. Thus, when evaluated at the SS:

$$
\begin{aligned}
& 0=-C_{m}\left[q v_{f, 01}+(1-q) v_{f, 02}\right]+C_{f}\left[q v_{m, 01}+(1-q) v_{m, 02}\right] \\
& 0=-C_{m}\left[(1-q) v_{f, 01}+q v_{f, 02}\right]+C_{f}\left[(1-q) v_{m, 01}+q v_{m, 02}\right]
\end{aligned}
$$

Solving for the reproductive values in (5.52), we obtain the following equalities:

$$
\frac{C_{m}}{C_{f}}=\frac{v_{m, 01}}{v_{f, 01}}=\frac{v_{m, 02}}{v_{f, 02}}
$$

From (5.53), we also see that:

$$
\begin{aligned}
\frac{v_{m, 02}}{v_{m, 01}} & =\frac{v_{f, 02}}{v_{f, 01}} \\
M R V R & =F R V R
\end{aligned}
$$

That is, the male and female reproductive value ratios are equal at any interior SS.

\section{A.2 Boundary SS (Type 2-5)}

For an boundary SS, only one component of the selection gradient (5.51) is 0 . The other component is either positive or negative depending on the specific type of boundary SS (Figure 5-2).

- For a Type $2 \mathrm{SS}, \frac{d \lambda^{\prime}}{d s_{1}^{\prime}}=0$. From (5.51), we see that:

$$
C_{m}\left[q v_{f, 01}+(1-q) v_{f, 02}\right]=C_{f}\left[q v_{m, 01}+(1-q) v_{m, 02}\right]
$$

Also for a Type $2 \mathrm{SS}, \frac{d \lambda^{\prime}}{d s_{2}^{\prime}}<0$. Solving (5.55) for $v_{m, 01}$ or $v_{m, 02}$ and substituting the result into the expression for $\frac{d \lambda^{\prime}}{d s_{2}^{\prime}}$ in $(5.51)$, we obtain:

$$
\begin{aligned}
\frac{d \lambda^{\prime}}{d s_{2}^{\prime}} & =\frac{2 q-1}{q}\left(C_{f} v_{m, 02}-C_{m} v_{f, 02}\right) \\
& =\frac{2 q-1}{q-1}\left(C_{f} v_{m, 01}-C_{m} v_{f, 01}\right) \\
& <0
\end{aligned}
$$


Because $0.5 \leq q \leq 1$, the conditions in (5.56) become:

$$
\begin{aligned}
& \frac{v_{m, 02}}{v_{f, 02}}<\frac{C_{m}}{C_{f}} \\
& \frac{v_{m, 01}}{v_{f, 01}}>\frac{C_{m}}{C_{f}}
\end{aligned}
$$

Combining the two inequalities in (5.57) and noting that all the quantities are positive, we obtain:

$$
\begin{aligned}
\frac{v_{m, 02}}{v_{m, 01}} & <\frac{v_{f, 02}}{v_{f, 01}} \\
M R V R & <F R V R
\end{aligned}
$$

- For a Type $3 \mathrm{SS}, \frac{d \lambda^{\prime}}{d s_{1}^{\prime}}=0$ as well, but $\frac{d \lambda^{\prime}}{d s_{2}^{\prime}}>0$. We accordingly apply (5.57) with the inequalities flipped to obtain:

$$
\begin{aligned}
\frac{v_{m, 02}}{v_{m, 01}} & >\frac{v_{f, 02}}{v_{f, 01}} \\
M R V R & >F R V R
\end{aligned}
$$

- For a Type $4 \mathrm{SS}, \frac{d \lambda^{\prime}}{d s_{2}^{\prime}}=0$. From (5.51), we see that:

$$
C_{m}\left[(1-q) v_{f, 01}+q v_{f, 02}\right]=C_{f}\left[(1-q) v_{m, 01}+q v_{m, 02}\right]
$$

Also for a Type $4 \mathrm{SS}, \frac{d \lambda^{\prime}}{d s_{1}^{\prime}}<0$. Using methods analogous to those above, it can be shown that:

$$
\begin{aligned}
\frac{v_{m, 02}}{v_{m, 01}} & >\frac{v_{f, 02}}{v_{f, 01}} \\
M R V R & >F R V R
\end{aligned}
$$

- For a Type $5 \mathrm{SS}, \frac{d \lambda^{\prime}}{d s_{2}^{\prime}}=0$ and $\frac{d \lambda^{\prime}}{d s_{1}^{\prime}}>0$, which yields:

$$
\begin{aligned}
\frac{v_{m, 02}}{v_{m, 01}} & <\frac{v_{f, 02}}{v_{f, 01}} \\
M R V R & <F R V R
\end{aligned}
$$

All these results are summarized in Table 5.1. 


\section{B Stability of $2 \mathrm{D}$ singular strategies}

The evolutionary and convergence stability of a singular strategy is characterized using the local second derivatives of the invasion fitness (5.15). We have previously showed second derivatives calculations for a single evolving sex ratio in Shyu and Caswell (xxb). Though analogous calculations can be performed for vector-valued traits, the SS stability conditions are more stringent (Table 5.B.1).

\begin{tabular}{|c|c|c|}
\hline Type of stability & $\begin{array}{l}\text { Sufficient condition for } \\
\text { stability }\end{array}$ & $\begin{array}{l}\text { Sufficient condition for } \\
\text { instability }\end{array}$ \\
\hline Evolutionary stability & $\mathbf{H}$ is negative definite & $\mathbf{H}$ is positive definite \\
\hline Convergence stability & $\begin{array}{l}\text { VJ has only eigenvalues with } \\
\text { negative real parts }\end{array}$ & $\begin{array}{l}\text { VJ has at least one eigenvalue } \\
\text { with a positive real parts }\end{array}$ \\
\hline Strong convergence stability & $\mathbf{J}$ is negative definite & $\mathbf{J}$ is positive definite \\
\hline
\end{tabular}

Table 5.B.1: Conditions for the evolutionary and convergence stability of a vector-valued singular strategy. These conditions depend on the Hessian $\mathbf{H}$ of the invasion fitness (5.63), the Jacobian $\mathbf{J}$ of the selection gradient (5.64), and the mutational variance-covariance matrix $\mathbf{V}$ in the canonical equation (5.18) (Apaloo and Butler 2009, Leimar 2009).

\section{B.1 Evolutionary stability}

Evolutionary stability indicates that the SS cannot be invaded by any nearby mutants. It depends on $\mathbf{H}$, the Hessian matrix of the invasion fitness:

$$
\mathbf{H}=\frac{\partial^{2} \lambda^{\prime}}{\partial \mathbf{s}^{\prime} \partial \mathbf{s}^{\prime \top}}
$$

This expression can be calculated using the matrix calculus methods detailed in Shyu and Caswell (xxb, Section 3.3.1).

A SS $\mathbf{s}^{*}$ is evolutionarily stable if $\mathbf{H}$ is negative definite at $\mathbf{s}^{*}$ (Apaloo and Butler 2009). Many of the $\mathbf{H}$ matrices in our model are negative semidefinite or indefinite because of zero eigenvalues, leading to inconclusive stability results. Figure 5.B.1 shows several examples of the corresponding invasion fitness landscapes. In these cases, and many others in our model, $\mathbf{s}^{*}$ lies on a line of points with zero invasion fitness. This means that there are an infinite number of mutant sex ratio combinations that have the same fitness as $\mathbf{s}^{*}$.

This is similar to our results for a single evolving sex ratio (Shyu and Caswell $\mathrm{xxb}$ ), where $s^{*}$ lies on a zero isocline for which any mutant sex ratios have equal fitness. This type of SS is known as a selectively neutral or weak form ESS (Bull and Charnov 1988, Uyenoyama and Bengtsson 1982). Once the population reaches a weak form ESS, there is no selective pressure to evolve further, because no mutants have positive invasion fitness. However, since all mutants have the same fitness as the resident, they may potentially coexist at low levels. 
a) interior SS

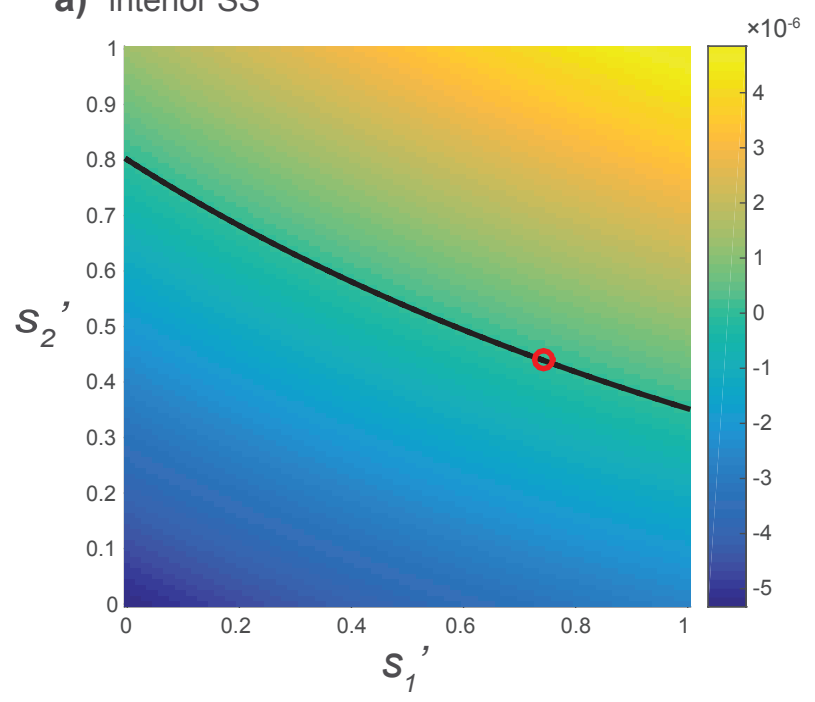

b) boundary SS

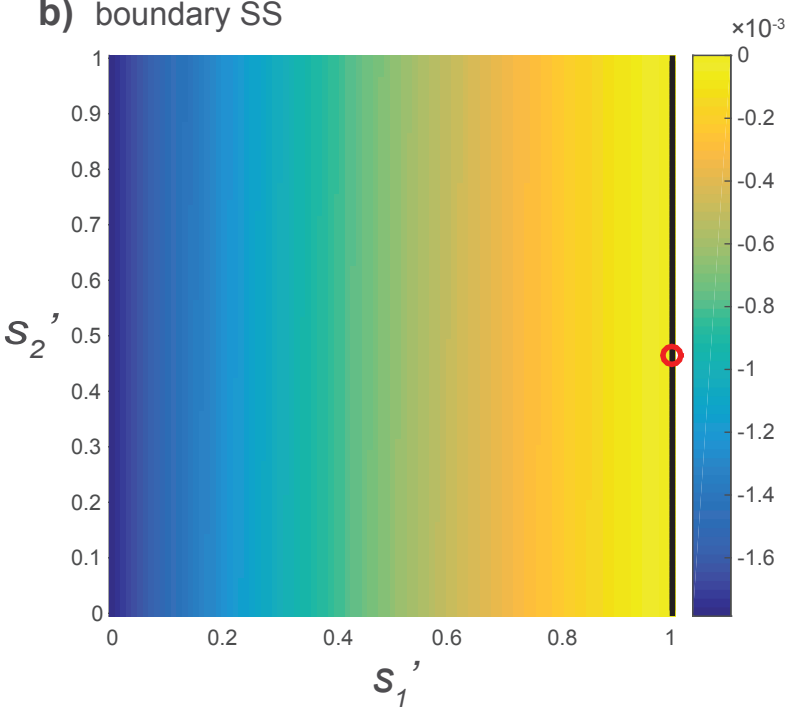

Figure 5.B.1: The invasion fitness (5.15) as a function of the mutant sex ratios $s_{1}^{\prime}$ (low quality females) and $s_{2}^{\prime}$ (high quality females). The resident population is at its SS sex ratio strategy $\mathbf{s}^{*}$. Red dots indicate the location of $\mathbf{s}^{*}$, while black lines indicate isoclines of zero invasion fitness (where the mutant and resident are equally fit). Plots are for the Case 2 (maternal quality) model with different values of $q$ (same as Figure 5-2). $C_{m}=C_{f}=0.5, c_{1}=0.1, c_{2}=0.9, R_{1}=10, R_{2}=20$; other parameters are as given in Table 5.1. a) Example of an interior SS (Type 1) for $q=0.65$. The eigenvalues of $\mathbf{H}$ are $-1.6 \times 10^{-6}$ and $2.9 \times 10^{-7}$. b) Example of a boundary SS (Type 5) for $q=0.8$. The eigenvalues of $\mathbf{H}$ are $-8.5 \times 10^{-4}$ and $2 \times 10^{-7}$.

\section{B.2 Convergence stability}

Convergence stability indicates that the SS is an evolutionary attractor that the trait will converge to through small mutations. It depends on $\mathbf{J}$, the Jacobian matrix of the selection gradient (Leimar 2009):

$$
\mathbf{J}=\mathbf{H}+\frac{\partial^{2}\left(\lambda^{\prime}-\lambda\right)}{\partial \mathbf{s}^{\prime} \partial \mathbf{s}^{\top}}
$$

Again, this expression can be calculated using the matrix calculus methods detailed in Shyu and Caswell (xxb, Section 3.3.2).

A SS $\mathbf{s}^{*}$ is convergence stable if $\mathbf{V J}$, the product of the mutational matrix from (5.18) and the Jacobian (5.64), has eigenvalues with negative real parts at $\mathbf{s}^{*}$. The SS is strongly convergence stable (stable for any smooth, symmetric, positive definite $\mathbf{V}$ ) if $\mathbf{J}$ is negative definite at $\mathbf{s}^{*}$ (Leimar 2009). Again, many of the $\mathbf{V J}$ and $\mathbf{J}$ matrices in our model are negative semidefinite or indefinite because of zero eigenvalues, leading to inconclusive stability results. Stability can be especially difficult to characterize for boundary SSs, which have limited directions for evolution.

Example eigenvalues of VJ are shown in Figure 5.B.2. Note that for boundary SSs, at least one diagonal entry of $\mathbf{V}$, as given by (5.20), will be 0 , resulting in zero eigenvalues of VJ. Zero 
eigenvalues appear to correspond to eigenvectors pointing towards the boundaries, while negative eigenvalues correspond to eigenvectors pointing along the boundaries.
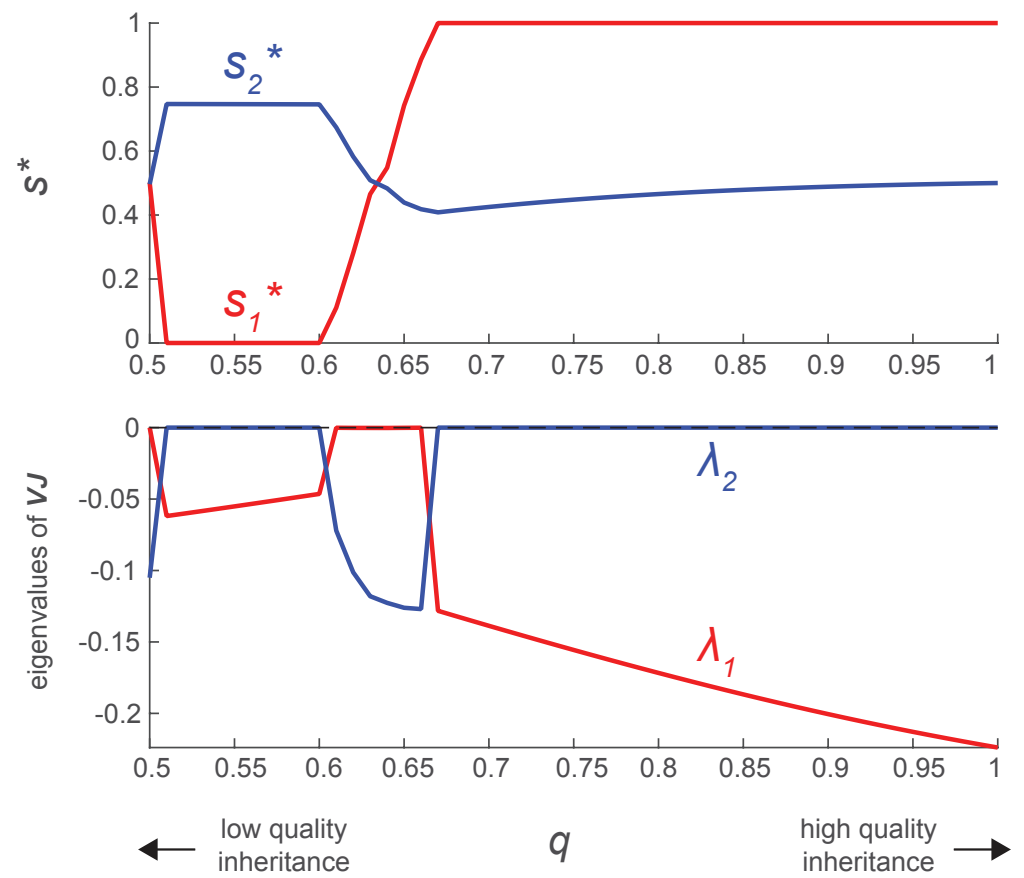

Figure 5.B.2: Eigenvalues $\lambda_{1}, \lambda_{2}$ of the matrix VJ, which is used to characterize convergence stability (Table 5.B.1). Model is the same as in Figure 5.B.2.

\section{Bibliography}

[1] Apaloo J, Butler S. 2009. Evolutionary stabilities in multidimensional-traits and several-species models. Evolutionary Ecology Research. 11: 637-650.

[2] Bull JJ, Charnov EL. 1988. How fundamental are Fisherian sex ratios? Oxford Surveys in Evolutionary Biology. 5: 96-135.

[3] Leimar O. 2009. Multidimensional convergence stability. Evolutionary Ecology Research. 11: 191-208.

[4] Uyenoyama MK, Bengtsson BO. 1979. Towards a genetic theory for the evolution of the sex ratio. Genetics. 93: 721-736. 


\section{Chapter 6}

\section{Concluding Remarks}

\subsection{Summary}

Sex and other types of population structure may have significant ecological and evolutionary effects, with ensuing implications for the management of resource, protected, and pest species alike. As a result, my overarching goal for this thesis was to develop a general modeling framework that could be used not only to explore the dynamics of two-sex, stage-structured populations, but also to analyze the evolution of traits in this explicitly demographic context.

In this thesis, I have shown how to formulate stage-classified, two-sex models using transition rate matrices (Chapter 2). These models have frequency-dependent dynamics that converge to an equilibrium stage structure with exponential population growth. The long-term growth rates that emerge determine the fitness of a given phenotype, and thus play an important role in evolutionary processes.

I then extended the evolutionary methods of adaptive dynamics to my demographic modeling framework. Using matrix calculus, I defined and derived key evolutionary quantities in my models, including the invasion fitness, selection gradient, and the second derivatives of growth rates. These second derivatives, in particular, have a broad range of ecological and evolutionary applications that I have also briefly explored (Chapter 4).

For the remainder of my thesis, I combined the two-sex modeling framework with matrix-based adaptive dynamics, to analyze sex ratio evolution in an explicitly demographic context. I used a series of two-sex population models to characterize potential evolutionary outcomes for sex ratio -both in the case of a single sex ratio (Chapter 3), and of a facultative sex ratio with two possible states (Chapter 5). In each case, I found and classified multiple evolutionary outcomes, including a prevalence of selectively neutral singular strategies.

Together, these results highlight the potential importance of sex and other population structure in both ecology and evolution. The two-sex model has lent some insight into the demographic factors that can cause different types of behavior. For sex ratio in particular, the outcomes that emerge often depend on properties that are sex- or stage-specific. 
In Chapter 2, for instance, population growth and equilibrium structure are modulated by multiple factors, including the extent to which mortality is male- or female-biased. Furthermore, populations may be more or less resilient to sex-biased harvest pressure depending on the properties of mated unions or harems. Factors like harem size and persistence affect not only unharvested population growth, but also population responses to sex-biased harvest. Models that do not distinguish between multiple sexes or union stages could not capture the nuances of these responses.

In some cases, the addition of demographic structure leads to results that are unexpected, or that even contradict those of models lacking such structure. Chapter 3 shows that the incorporation of a union stage enables sex-specific mortality to skew sex ratios in ways that were unanticipated by previous, non-demographic sex ratio theory. These results challenge the classic and widely-held belief that mortality after the period of parental investment cannot affect the primary sex ratio, especially when union productivity is poor.

The use of adaptive dynamics in a demographic context can also reveal interesting results about transient evolutionary dynamics. In Chapter 5, for example, the evolutionary trajectories given by the multidimensional canonical equation show that there are cases where sex ratios converge to a selectively neutral line of singular strategies. This potential for infinitely many sex ratio combinations may help explain the mixed results of empirical sex ratio studies.

\subsection{Extensions}

The modeling framework that I have developed can accommodate arbitrarily many stages and life cycles processes to represent complex life histories. The adaptive dynamics methods I presented can also be readily adapted to study the evolution of other traits. Because my models can incorporate any relevant structure (including multiple sexes, stages, and mated unions) into evolutionary projections, they can model population and evolutionary dynamics in a variety of populations.

One could, for instance, apply similar analyses to other examples of sex allocation, such as hermaphroditism (e.g., Charnov 1982) or environmental sex determination (e.g., Frank and Swingland 1988). My framework would also be well suited for modeling other cases of frequency-dependent selection in structured populations.

\subsubsection{Alternative mating strategies}

One such case is that of alternative mating strategies. In a "typical" mating system, one sex (usually males) competes by fighting for partners and other resources. Some males, however, avoid direct confrontation by surreptitiously mating with partners of the more dominant males. These alternative mating strategies have been observed in a wide variety of taxa, including insects, fish, amphibians, and mammals (e.g., Krebs and Davies 1993).

In Pacific salmon (Oncorhynchus species), for example, there are two alternative phenotypes for males (Gross 1985). Hooknose males are slower to mature, but larger and specialized for fighting 
competitors. Jack males, in contrast, are smaller, mature more quickly, and obtain mates by sneaking fertilizations during spawning. These phenotypes appear to be genetically predetermined, possibly through the Y-chromosome (Heath et al. 2002). Both are maintained within populations through negative frequency-dependent selection (Gross 1985), as an overabundance of either strategy would increase competition and reduce fitness.

Modeling alternative mating strategies may benefit from the two-sex, explicitly demographic framework, for this phenomenon contains clear examples of both stage structure (here, alternative male phenotypes) and sex structure (males, females, and mating interactions that vary between phenotypes). Recall that sex ratio evolution is also determined by negative frequency-dependent selection, as an overabundance of either sex similarly increases competition and reduces fitness. As a result, the evolution of alternative mating strategies parallels the evolution of sex ratio strategies, and could accordingly be analyzed using methods analogous to the ones shown here.

\subsubsection{Evolution under harvest}

The view that evolutionary change is slow and undetectable over conservation-relevant timescales has caused many managers to consider demographic traits as static, even in the face of shifting selective pressures. Population viability analyses (PVAs) and climate range shift models, for instance, frequently extrapolate population dynamics based on current trait values, without considering potential adaptations. Growing evidence, however, suggests that evolutionary considerations are relevant to sustainable long-term management. For example, rapid evolution on the scale of months to years has been observed in a variety of taxa (Ashley 2003), mostly in response to recent anthropogenic pressures.

Human-induced selection is especially important for harvested species. As harvest mortalities are often more severe and selective than natural mortalities, they may drive evolution in directions that would not occur under natural conditions - as well as directions unfavorable to harvesters. Lower fishery yields, for instance, have been attributed to selective exploitation of larger and older fish, which has induced decreases in body weight, length, and the age and size at maturity (e.g., Law 2000, Kuparinen 2007).

In the case of sex-biased harvest, hunters often target the phenotypes most favored for mating or fecundity (e.g., bigger body size, larger horns or tusks, etc.). The "unnatural" selection induced by such harvest may significantly alter population structure, reproductive strategies, body morphology, and developmental timing (e.g., Ashley 2003, Festa-Bianchet 2003, Allendorf and Hard 2009).

Determining how sex-biased harvest will affect traits like sex ratio, and ultimately impact population growth, may help inform harvest regulations and management programs. As alluded to at the end of Chapter 2, my two-sex modeling framework can be used to study evolutionary responses to changing environmental conditions or human activities, including sex-biased harvest. Harvest effects could, for instance, be framed in terms of sex-specific mortality, as I explored in 
Case 2 (mortality during parent investment) and Case 3 (mortality after parental investment) of Chapter 3.

As an additional example, consider the application of evolutionary singular strategy calculations for single sex ratios (analogous to those described in Chapter 3, Case 3 for differential adult mortality) to the "polygynous harems" model with sex-biased harvest (described in Chapter 2). As in Chapter 2, assume that only adults (both in and out of unions) are harvested, and that a proportion $s_{h}$ of harvest effort is focused on males. Figure 6-1 compares how the primary sex ratio evolves both before and after harvest.

Figure 6 -1a shows the evolutionarily singular sex ratios $s^{*}$ that result when the population is unharvested. In this case, sex ratio evolution depends only on population properties - namely, the harem size $h$ and divorce rate $d$. Persistent $(d=0)$ and monogamous $(h=1)$ unions do not deviate much from the equal sex ratio $s^{*}=0.5$. However, populations with large harems (large $h$ ) appear to evolve male-biased sex ratios $\left(s^{*}>0.5\right)$. This result is somewhat counterintuitive, as populations with large harems would seemingly benefit from more females than males.

Figures 6-1b-d show how $s^{*}$ changes from Figure 6-1a under sex-biased harvest. Female-biased harvest causes $s^{*}$ to decrease (become more female-biased), and affects low $d$, low $h$ populations the most. In contrast, male-based harvest causes $s^{*}$ to increase (become more male-biased), and affects low $d$, high $h$ populations the most. The sex ratio shifts induced by unbiased harvest have smaller magnitudes, but varied directions; unbiased harvest would, for instance, increase $s^{*}$ in high $d$, low $h$ populations, but decrease it in low $d$, high $h$ populations.

Evolutionary changes in sex ratio also affect population growth rates. Figure 6-2 shows the change in the population growth rate $\lambda$ due to evolution of the primary sex ratio (from the equal sex ratio $s=0.5$ to the evolutionarily singular sex ratio $s=s^{*}$ ). In most cases, $\lambda$ decreases as $s$ evolves to $s^{*}$, regardless of whether the population is being harvested or not. In some cases, however, $\lambda$ actually increases in the presence of sex ratio evolution - in small $d$ populations under male-biased harvest, for example.

Overall, these results indicate that sex ratio evolution can affect population growth in different ways, depending on factors like mating system parameters $(d, h)$ or harvest bias. Evolution over management-relevant timescales may accordingly influence population responses to harvest.

\section{Bibliography}

[1] Allendorf FW, Hard JJ. 2009. Human-induced evolution caused by unnatural selection through harvest of wild animals. Proceedings of the National Academy of Sciences. 106: 9987-9994.

[2] Ashley MV, Willson MF, Pergams ORW, O'Dowd DJ, Gende SM, Brown JS. 2003. Evolutionarily-enlightened management. Biological Conservation. 111: 115-123.

[3] Charnov EL. 1982. The theory of sex allocation. Princeton: Princeton University Press. 

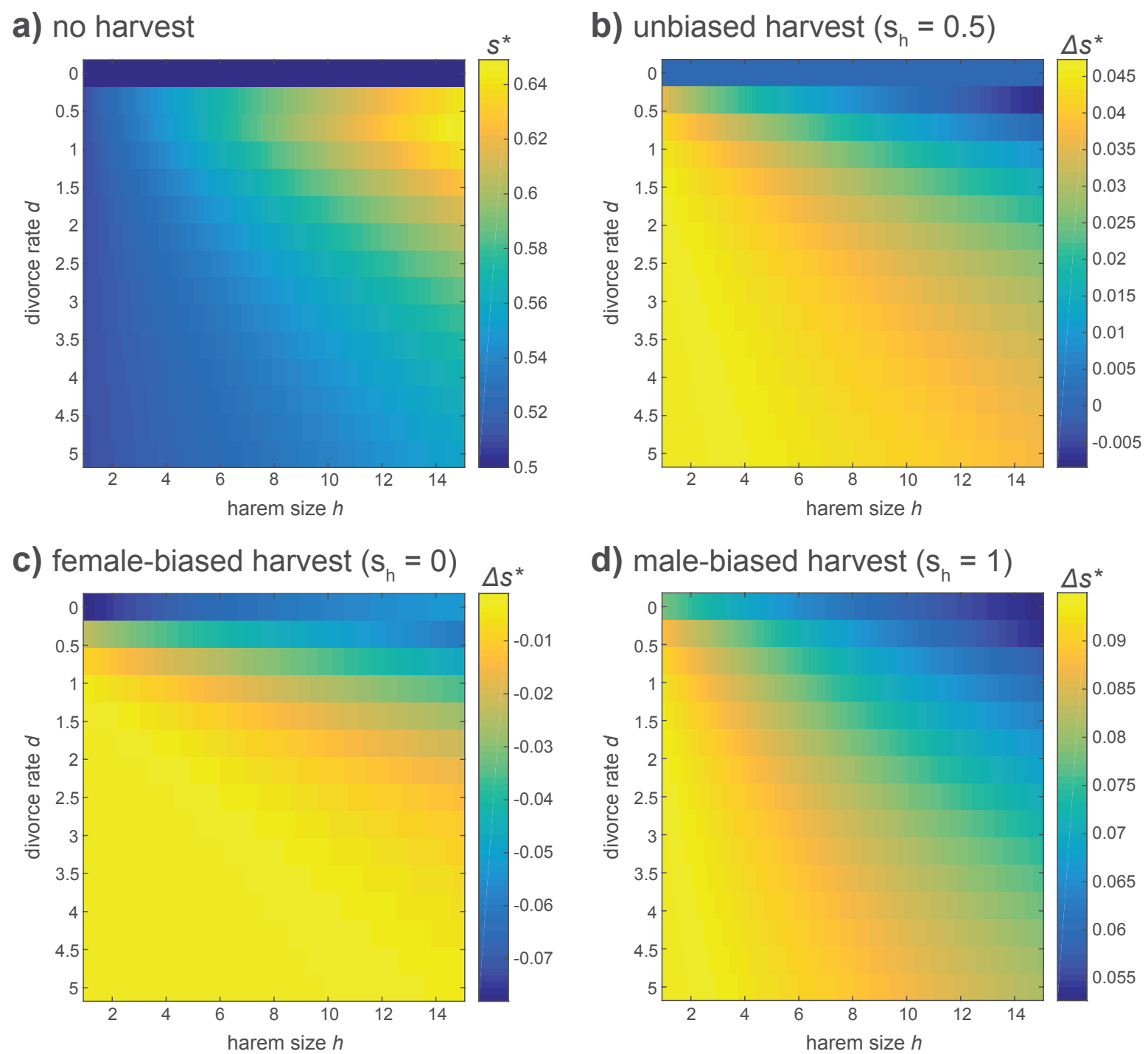

Figure 6-1: Evolutionarily singular strategies of the primary sex ratio $s^{*}$ in the polygnous harems model (Chapter 2), as functions of the population divorce rate $d$ and harem size $h$. a) SS sex ratios in an unharvested population; change in the sex ratio from Figure 6-1a due to $b$ ) unbiased harvest $\left.\left(s_{h}=0.5\right), c\right)$ female-biased harvest $\left(s_{h}=0\right)$, or $\left.d\right)$ male-biased harvest $\left(s_{h}=1\right)$. Negative changes indicate that $s^{*}$ is decreasing (becoming more female-biased), while positive changes indicate that $s^{*}$ is increasing (becoming more male-biased). Other parameters are fixed at $\mu_{m 1}=\mu_{f 1}=0.5, \mu_{m 2}=$ $\mu_{f 2}=0.1, \alpha_{m}=\alpha_{f}=0.5, s_{1}=0.5, k=20, E=1$. 
a) no harvest

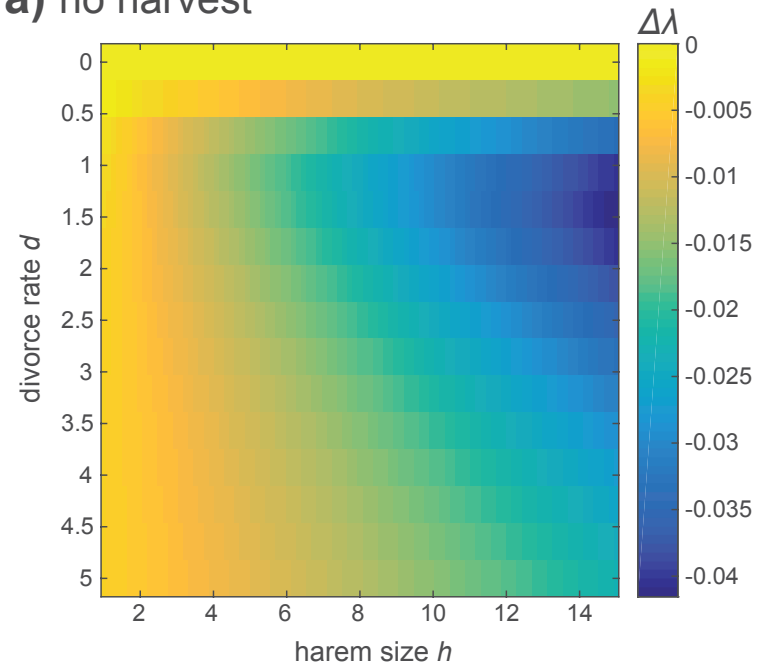

c) female-biased harvest $\left(\mathrm{s}_{\mathrm{h}}=0\right)$

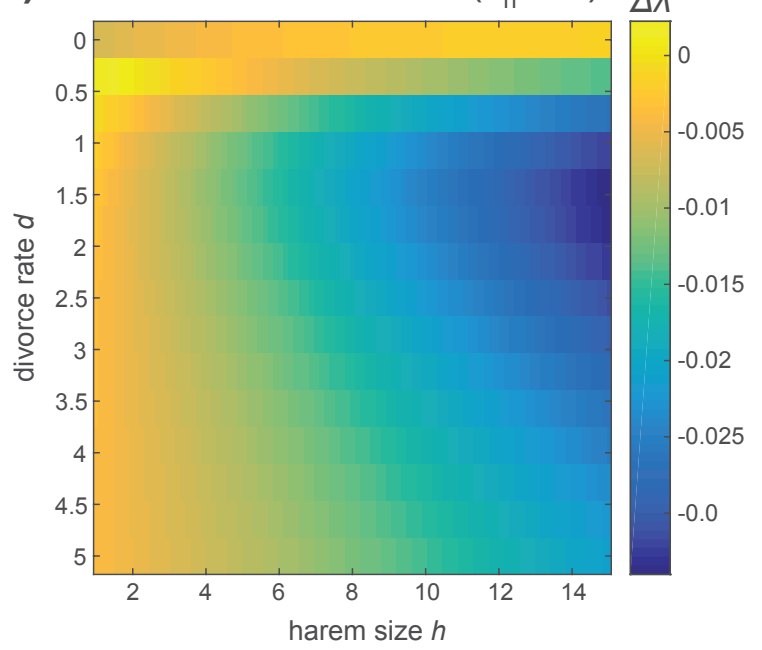

b) unbiased harvest $\left(\mathrm{s}_{\mathrm{h}}=0.5\right)$

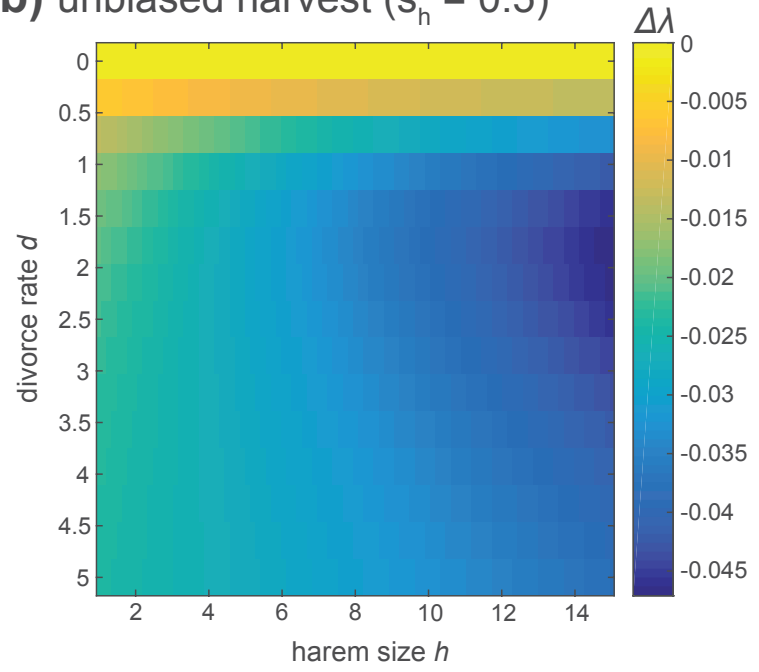

d) male-biased harvest $\left(s_{h}=1\right)$

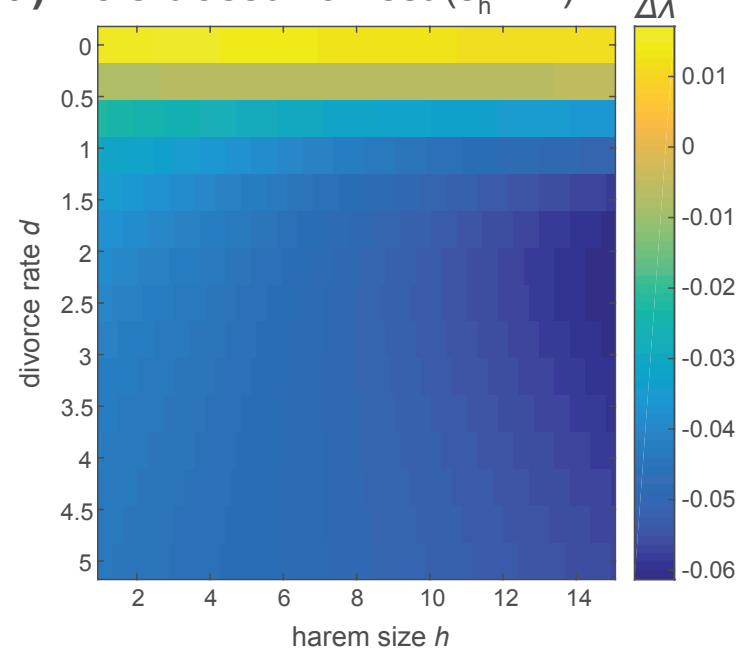

Figure 6-2: Change in the population growth rate $\lambda$, due to evolution from $s=0.5$ to $s=s^{*}$, when there is a) no harvest $(E=0), b)$ unbiased harvest $\left.\left(E=0.5, s_{h}=0.5\right), c\right)$ female-biased harvest $\left(E=0.5, s_{h}=0\right)$, or $\left.d\right)$ male-biased harvest $\left(E=0.5, s_{h}=1\right)$. The model and resulting $s^{*}$ values are the same as in Figure 6-1. 
[4] Festa-Bianchet M. 2003. Exploitative wildlife management as a selective pressure for life-history evolution of large mammals. In Animal Behavior and Wildlife Conservation (eds Festa-Bianchet M, Apollonio M). Washington, D.C.: Island Press. pp. 191-210.

[5] Frank, SA, Swingland, IR. 1988. Sex ratio under conditional sex expression. Journal of Theoretical Biology. 135: 415-418.

[6] Gross MR. 1985. Disruptive selection for alternative life histories in salmon. Nature. 313: 47-48.

[7] Heath DD, Rankin L, Bryden CA, Heath JW, Shrimpton JM. 2002. Heritability and Y-chromosome influence in the jack male life history of chinook salmon (Oncorhynchus tshawytscha). Heredity. 89: 311-317.

[8] Krebs, JR, Davies, NB. 1993. An Introduction to Behavioural Ecology. 3rd ed. Oxford: Blackwell Publishing.

[9] Kuparinen A, Merilä J. 2007. Detecting and managing fisheries-induced evolution. TRENDS in Ecology and Evolution. 22: 652-659.

[10] Law R. 2000. Fishing, selection, and phenotypic evolution. ICES Journal of Marine Science. 57: 659-669. 